\title{
DYNAMIC CHARACTERIZATION AND VIBRATION SERVICEABILITY ASSESSMENT OF A HIGHLY TRAFFICKED, LIVELY PEDESTRIAN BRIDGE
}

\author{
A Thesis \\ Presented to \\ the faculty of the School of Engineering and Applied Science at \\ University of Virginia
}

in partial fulfillment

of the requirements for the degree

Master of Science

by

SALMAN USMANI

May

2016 


\section{APPROVAL SHEET}

The thesis

is submitted in partial fulfillment of the requirements

for the degree of

Master of Science

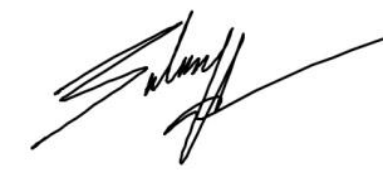

AUTHOR

The thesis has been read and approved by the examining committee:

Osman E. Ozbulut

Advisor

Devin K. Harris

Thomas T. Baber

Accepted for the School of Engineering and Applied Science:

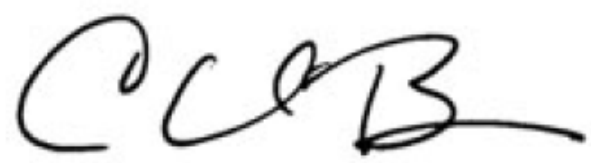

Craig H. Benson, Dean, School of Engineering and Applied Science 


\begin{abstract}
Pedestrian bridges represent a class of structures that are not typically heavily loaded, but may experience significant vibrations during operation due to pedestrian traffic and wind loads. To investigate the effect of these vibrations, pedestrian bridges can be characterized based on their dynamic properties such as natural frequencies, mode shapes and damping ratios. Existing design codes address the vibration levels either by ensuring the critical frequency ranges associated with typical pedestrian bridges are outside the fundamental frequencies of the structure or by restricting the maximum accelerations below the limits for pedestrian comfort. This research study discusses vibration serviceability assessment of a highly trafficked, lively pedestrian bridge based on the experimental tests and numerical analysis. The selected bridge is an approximately 190-ft long three-span steel structure with a continuous reinforced concrete slab supported on two longitudinal steel girders.

First, a finite element model of the pedestrian bridge was developed to setup key instrumentation parameters for the field tests. Experimental tests consisting of impact hammer tests, ambient vibration tests and pedestrian interaction tests were then conducted to obtain the modal characteristics of the structure. It was observed that the fundamental frequency of the bridge in the vertical direction obtained through field tests was within the critical range described by available design guidelines. This required further analysis to assess the performance of the bridge relative to the maximum acceleration threshold. In addition to the peak dynamic response obtained from the pedestrian interaction tests, peak acceleration values based on current design guidelines were computed analytically and compared to the comfort limits. Results from the study suggest that the footbridge possesses satisfactory serviceability performance under low and dense traffic conditions, but the comfort level was classified as minimum under very dense traffic loads.
\end{abstract}




\section{DEDICATION}

To my grandfather, Muhammad Ibrahim Usmani, a man of profound humility and unparalleled wisdom. 


\section{ACKNOWLEDGEMENTS}

I would like to thank the following people who helped me in accomplishing the formidable task of completing my master's thesis.

Dr. Osman E. Ozbulut for giving me the opportunity to work on such an exciting project. Your continued guidance, support and unwavering faith in my journey through thick and thin has indeed been the prime factor of the successful completion of my degree at UVa. Your depth of knowledge of the studied subject coupled with your great attitude towards teaching makes you a remarkable mentor and advisor. It has indeed been a privilege to have been one of your students, for which I will always be grateful.

Dr. Devin K. Harris for serving as the chair of my committee and for being my secondary advisor. Your guidance and assistance in the topic of bridge testing was vital in my work. Your leadership and management during my research project was a key element in the successful achievement of my set targets.

Dr. Thomas T. Baber for serving on my committee and for being an excellent resource and teacher on the topic of structural dynamics. Your wealth of knowledge and experience in the studied topic made for an invigorating learning experience for me. It has been a privilege learning from you by tapping into the vast source of knowledge you possess.

Dr. Jose P. Gomez for being a truly inspirational mentor and guide through my time at UVa. Your breadth of knowledge in steel and concrete design magnified by your expertise in the industry makes you an excellent resource to learn from. I greatly appreciate your advice and support.

Mohamad Alipour for being a great teammate. Your depth of knowledge in core civil engineering subjects, sincere devotion to research, acute planning and management skills topped with your great attitude makes you an excellent team player. Your constant support and guidance in my research was definitely the key element of success in my work at UVa.

Muhammad M. Sherif for being a friend, a teacher and most importantly a great motivator. Your achievements in research to date and your profound knowledge of a range of topics let alone structural engineering is nothing short of remarkable. Your knack of teaching, research prowess and your genuinely helpful and caring attitude showed me the true meaning of an education. 


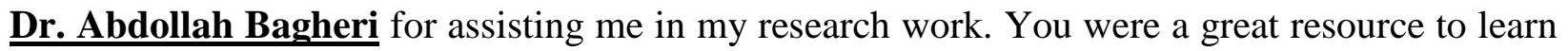
from especially in the topics of structural dynamics and modal analysis.

Mark Saliba for mentoring me throughout my masters and for teaching me about field testing equipment. You were an exceptional resource for help and made yourself available despite your busy schedule. I am grateful for your continued support and guidance and look forward to it in the future as well.

Jesse Sipple, Jacob Villa, Andrei Ramniceanu and Vahid Farmehini for being the technical guides and mentors of my research work. Your technical expertise in laboratory equipment and field testing techniques helped me in resolving numerous issues I had with my research.

Current and Ex Graduate Students including Sherif Daghash, Evelina Khakimova, Julia Carroll, Mohamed Morsy, Bakinam Essawy, Mohamad Amine, Lauren Bolton, Matthew Reardon, Mehrdat Shafei, Ethan Bradshaw, Zhangfan Jiang, Baikuntha Silwal, Lizzie Engel and Anna Smith. I greatly appreciate all your help and company during my time as a graduate student. Your presence in the graduate office made for a great learning environment.

My Beloved Parents, Sisters and Aunt for your love, support and warmth during times of joy and times of need. You have been the leading proponents for my education and career. Thank you for everything. 


\section{NOMENCLATURE}

\begin{tabular}{|c|c|}
\hline ABBREVIATION & DESCRIPTION \\
\hline AASHTO & American Association of State Highway and Transportation Officials \\
\hline AISC & American Institute of Steel Construction \\
\hline BSI & British Standards Institution \\
\hline BDI & Bridge Diagnostics Inc. \\
\hline CEN & European Committee for Standardization \\
\hline $\mathrm{CL}$ & Comfort Level \\
\hline DAQ & Data acquisition \\
\hline DFT & Discrete Fourier Transform \\
\hline DLF & Dynamic Load Factors \\
\hline EFDD & Enhanced Frequency Domain Decomposition \\
\hline EMA & Experimental Modal Analysis \\
\hline FDD & Frequency Domain Decomposition \\
\hline FEA & Finite Element Analysis \\
\hline FFT & Fast Fourier Transform \\
\hline FRF & Frequency Response Function \\
\hline HIVOSS & Human Induced Vibration of Steel Structures \\
\hline LRFD & Load and Resistance Factor Design \\
\hline MAC & Modal Assurance Criteria \\
\hline MDOF & Multi Degree of Freedom \\
\hline ODS & Operational Deflection Shapes \\
\hline OMA & Operational Modal Analysis \\
\hline PSD & Power Spectral Density \\
\hline SDOF & Single Degree of Freedom \\
\hline SVD & Singular Value Decomposition \\
\hline $\mathrm{TC}$ & Traffic Class \\
\hline
\end{tabular}




\begin{tabular}{|c|c|}
\hline SYMBOL & DESCRIPTION \\
\hline $\mathrm{k}$ & Stiffness \\
\hline $\mathrm{m}$ & Mass \\
\hline $\mathrm{c}$ & Damping \\
\hline $\mathrm{u}$ & Displacement \\
\hline$\phi$ & Modal vector \\
\hline $\mathrm{q}_{\mathrm{n}}$ & Modal coordinate \\
\hline$\omega_{n}$ & Natural frequency \\
\hline$\omega_{d}$ & Damped natural frequency \\
\hline$\zeta$ & Damping ratio \\
\hline $\mathrm{R}_{\mathrm{xx}}$ & Auto-correlation function \\
\hline $\mathrm{R}_{\mathrm{xy}}$ & Cross-correlation function \\
\hline$S_{x x}$ & Power spectral density \\
\hline$S_{x y}$ & Cross power spectral density \\
\hline$G_{x x}$ & One sided power spectral density \\
\hline$G_{x y}$ & One sided cross power spectral density \\
\hline$\psi$ & Reduction coefficient \\
\hline$\gamma$ & Coherence \\
\hline$\delta$ & Logarithmic decrement \\
\hline$\lambda_{k}$ & Pole \\
\hline$R_{k}$ & Residue \\
\hline$\Delta$ & Deflection \\
\hline $\mathrm{E}$ & Modulus of elasticity \\
\hline $\mathrm{I}$ & Moment of inertia \\
\hline $\mathrm{n}$ & Number of pedestrians \\
\hline $\mathrm{d}$ & Pedestrian density \\
\hline $\mathrm{a}$ & Acceleration \\
\hline $\mathrm{U}$ & Singular vector \\
\hline $\mathrm{S}$ & Singular values \\
\hline
\end{tabular}




\section{TABLE OF CONTENTS}

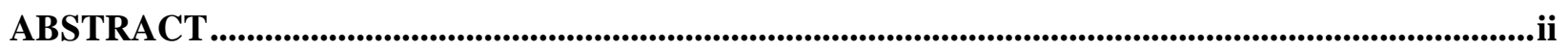

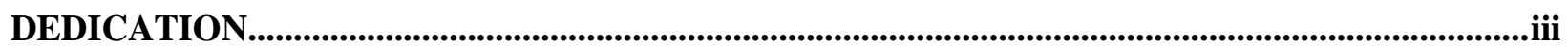

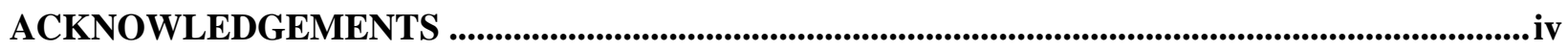

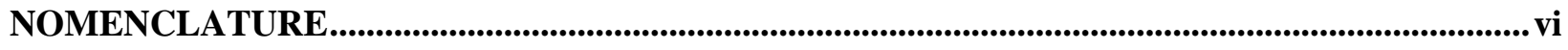

TABLE OF CONTENTS .................................................................................................................................................viii

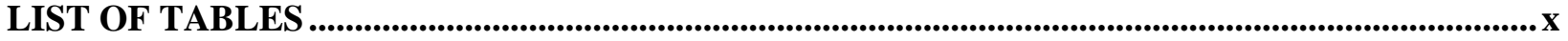

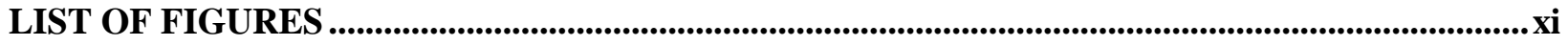

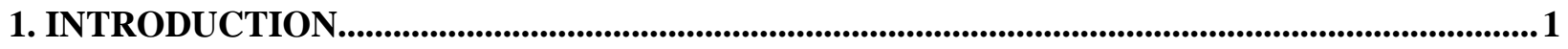

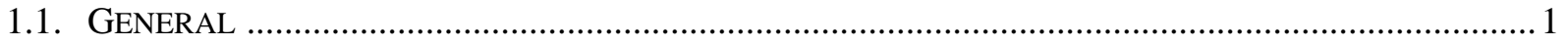

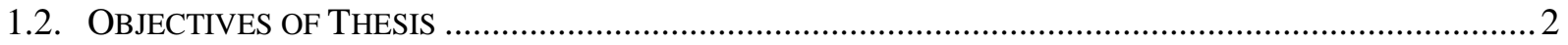

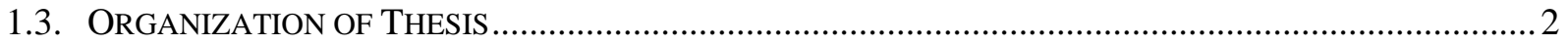

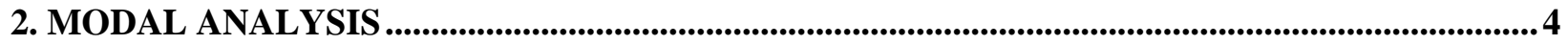

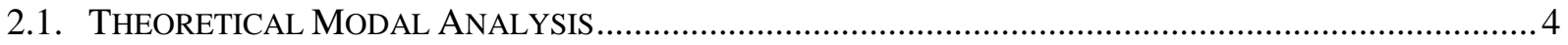

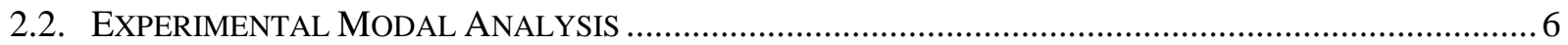

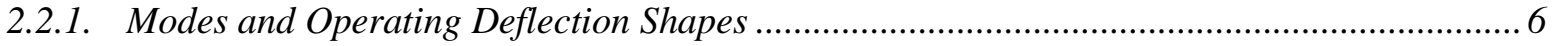

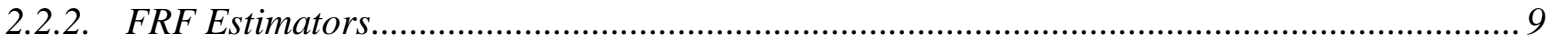

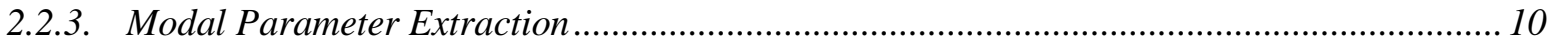

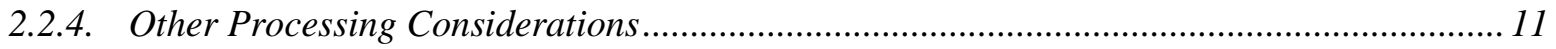

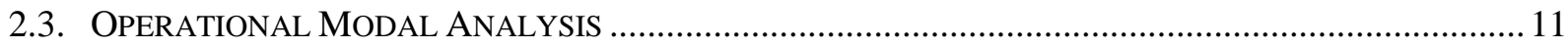

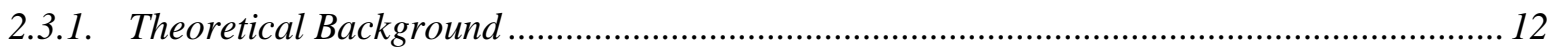

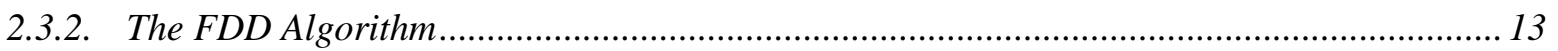

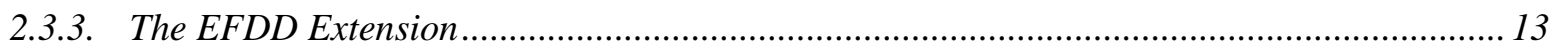

3. DESIGN GUIDELINES FOR SERVICEABILITY ASSESSMENT ................................................15

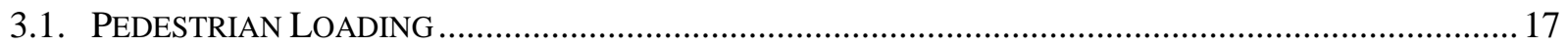

3.2. AASHTO LRFD GUIDE SPECIFICATIONS FOR THE DESIGN OF ................................................. 18

3.3. AisC SteEl DeSign Guide No. 11 (Floor Vibrations due to Human ACTiVity) ................ 19

3.4. HIVOSS (HUMAN INDUCED VIBRATIONS OF STEEL STRUCTURES) (HIVOSS, 2007) .................21

3.5. SÉTRA (ASSESSMENT OF VIBRATIONAL BEHAVIOR OF............................................................ 24

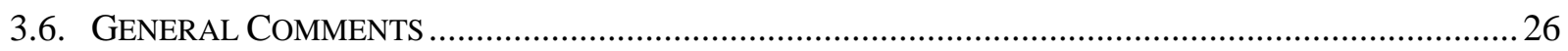




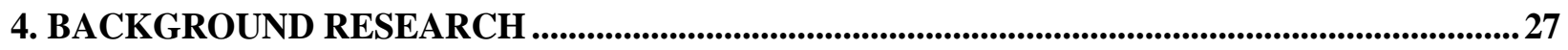

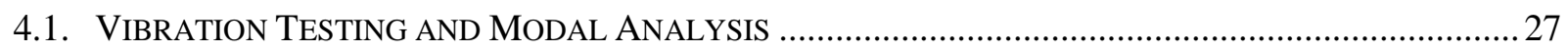

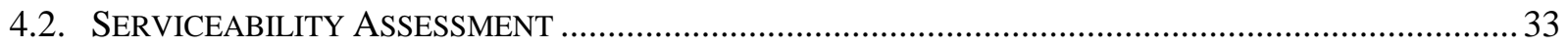

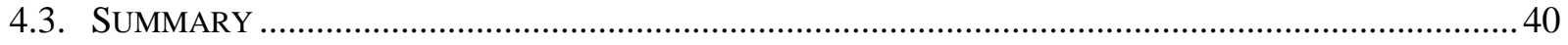

5. DYNAMIC CHARACTERIZATION OF RUFFNER FOOTBRIDGE .......................................42

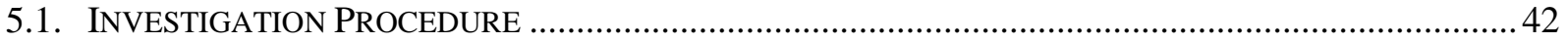

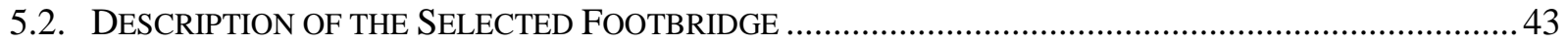

5.3. PREliminary Finite ElEMENT ANAlysis (FEA) (GHEITASI ET AL., 2016) .............................. 44

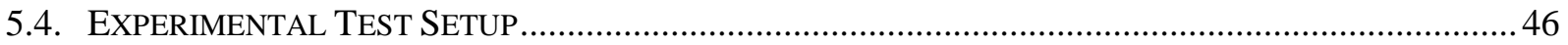

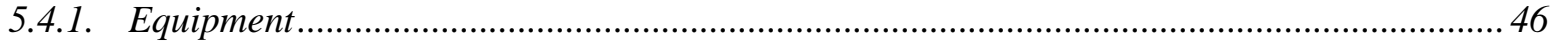

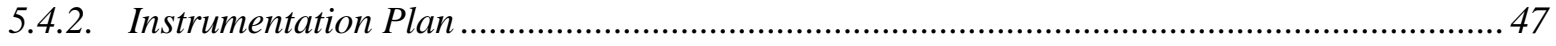

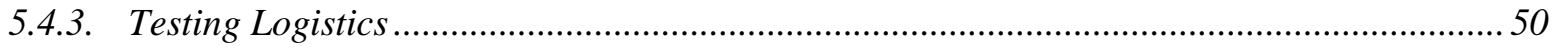

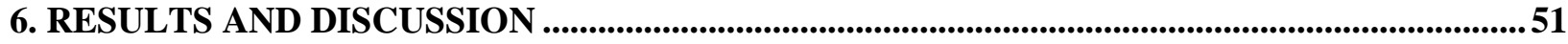

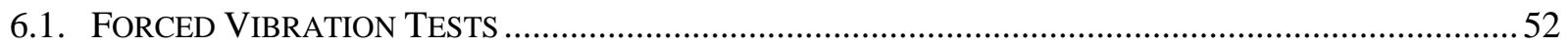

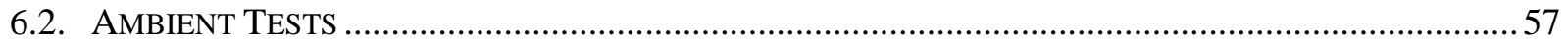

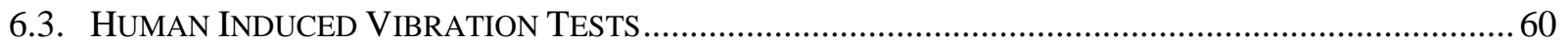

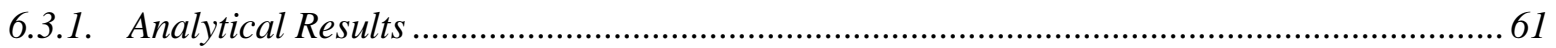

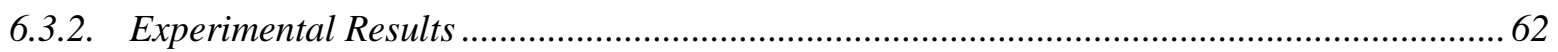

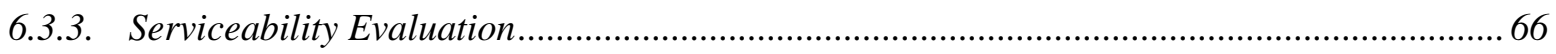

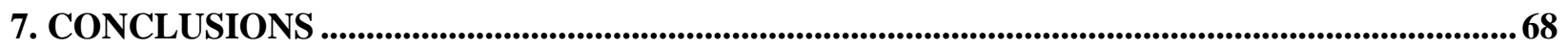

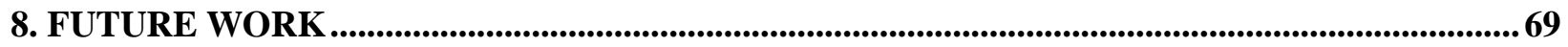

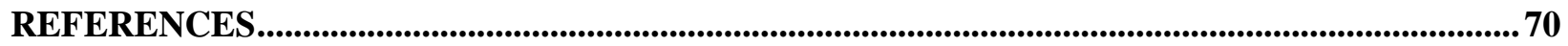

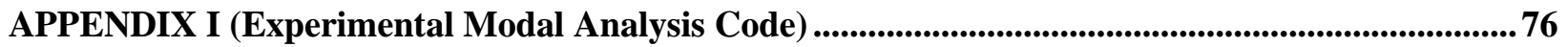

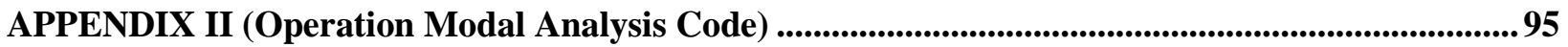

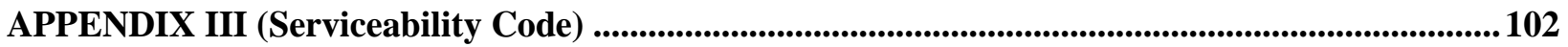

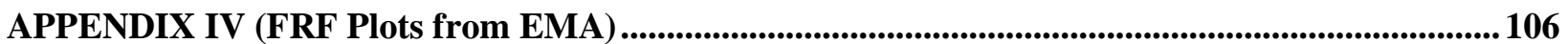




\section{LIST OF TABLES}

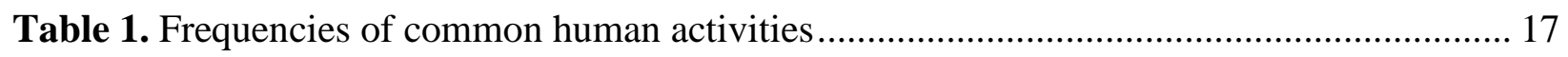

Table 2. Range of Frequencies by Design Codes (AASHTO, 2009; HIVOSS ........................... 18

Table 3. AASHTO LRFD Frequency Limit ....................................................................... 18

Table 4. Recommended Fourier Coefficients for Loading Model .............................................. 20

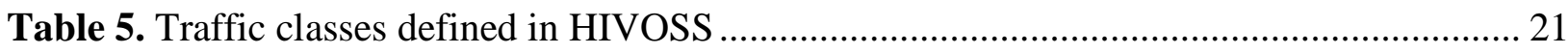

Table 6. Comfort levels defined in HIVOSS (*Limit value defined in SÉTRA guidelines) ....... 22

Table 7. Load cases to select for dynamic analysis..................................................................... 25

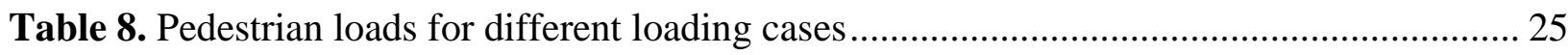

Table 9. Frequencies and modes of vibrations derived from the preliminary finite .................... 45

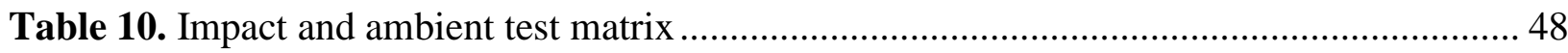

Table 11. Walking serviceability test matrix ....................................................................... 48

Table 12. Jumping serviceability test matrix ………………………………………….......... 49

Table 13. Summary of obtained natural frequencies.............................................................. 54

Table 14. Complete list of picked natural frequencies and damping ratios ................................. 54

Table 15. Obtained mode shapes for different impact points ..................................................... 57

Table 16. Natural frequencies, damping and MAC for summer and winter ................................ 58

Table 17. Obtained mode shapes in the summer and winter..................................................... 59

Table 18. MAC values for forced and ambient vibration tests ................................................... 59

Table 19. Deck and ambient temperatures during testing .......................................................... 60

Table 20. Analytical results for peak acceleration response ........................................................ 62

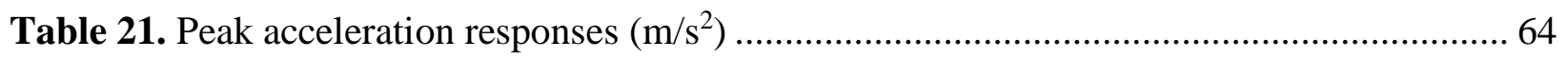

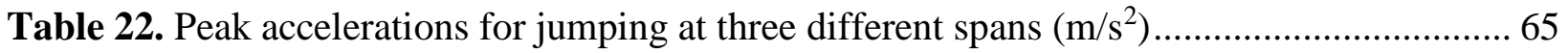




\section{LIST OF FIGURES}

Figure 1. Frequency Response Function Types .................................................................... 7

Figure 2. SDOF Peak Amplitude Method (Richardson, 1997)……………………................ 10

Figure 3. Serviceability Assessment Flowchart (Grandić, 2015) .............................................. 16

Figure 4. International Standardization Organization (ISO) Peak Acceleration Limits ............. 19

Figure 5. Reduction coefficient $\psi$ for (a) vertical and (b) transverse vibrations according ........ 23

Figure 6. Reduction coefficient for (a) vertical and (b) transverse vibrations ............................ 25

Figure 7. Ruffner Bridge a) View from Bavarro Hall (South West Side); b) View from ........... 43

Figure 8. (a) Cross bracing, (b) Girder connection between each span, (c) View of the ............ 44

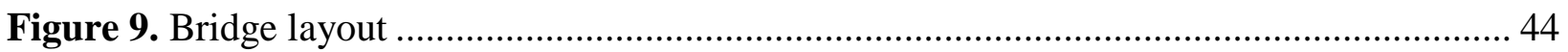

Figure 10. Ambient and impact tests instrumentation scheme ……............................................. 47

Figure 11. Serviceability first part tests instrumentation scheme .............................................. 48

Figure 12. Serviceability second part tests instrumentation scheme …..................................... 49

Figure 13. (a) Location 4 of accelerometer, (b) Node and base station shown on the left ......... 49

Figure 14. (a) Serviceability test with 9 people, (b) Impact hammer test.................................... 50

Figure 15. (a) Power fluctuation and (b) resolved ambient data for sensor 7 ............................. 51

Figure 16. (a) Low pass filter at $30 \mathrm{~Hz}$, (b) High pass filter at $0.5 \mathrm{~Hz}$..................................... 51

Figure 17. Sample impact hammer plot (top) and acceleration data from sensor 12 …............. 52

Figure 18. Frequency response function and coherence $\mathrm{H} 1 \& \mathrm{H} 2$ for impact location at 3 ....... 54

Figure 19. (a) Imaginary FRF plot for sensor 4, (b) Imaginary FRF plot for sensor 12 .............. 56

Figure 20. SVD plot of ambient test in winter ...................................................................... 57

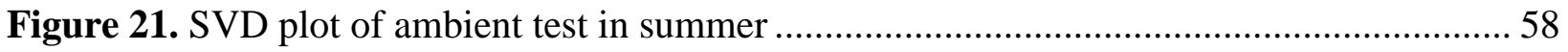

Figure 22. Marked accelerometer locations (regeneration of Figure 11) ................................... 63

Figure 23. Vertical (V3) and transverse (T3) accelerations due to walk at different frequencies 63

Figure 24. Vertical acceleration time histories at V3 during random flow of pedestrian tests.... 64

Figure 25. Marked accelerometer locations (regeneration of Figure 12) ................................... 65

Figure 26. Vertical time histories of V1, V4 and V6 sensors during a jumping motion .............. 65

Figure 27. Evaluation of pedestrian-induced dynamic response ............................................... 66

Figure 28. FRF from impact at location 3 and measurement at location 1 ............................... 106

Figure 29. FRF from impact at location 3 and measurement at location 2 ............................. 106 
Figure 30. FRF from impact at location 3 and measurement at location 3 .......................... 107

Figure 31. FRF from impact at location 3 and measurement at location 4 ........................... 107

Figure 32. FRF from impact at location 3 and measurement at location 5 .......................... 108

Figure 33. FRF from impact at location 3 and measurement at location 6........................... 108

Figure 34. FRF from impact at location 3 and measurement at location 7 ........................... 109

Figure 35. FRF from impact at location 3 and measurement at location 8 ........................... 109

Figure 36. FRF from impact at location 3 and measurement at location 9 ........................... 110

Figure 37. FRF from impact at location 3 and measurement at location $10 \ldots \ldots \ldots \ldots \ldots \ldots \ldots \ldots . . . . . . . . . . . . .110$

Figure 38. FRF from impact at location 3 and measurement at location $11 \ldots \ldots \ldots \ldots \ldots \ldots \ldots \ldots . . . . . . . . . . . .111$

Figure 39. FRF from impact at location 3 and measurement at location 12 ......................... 111

Figure 40. FRF from impact at location 3 and measurement at location 13 ......................... 112

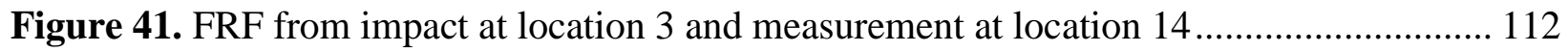

Figure 42. FRF from impact at location 3 and measurement at location 15 ......................... 113

Figure 43. FRF from impact at location 3 and measurement at location 16........................ 113

Figure 44. FRF from impact at location 3 and measurement at location 17 .......................... 114

Figure 45. FRF from impact at location 3 and measurement at location $18 \ldots \ldots \ldots \ldots \ldots \ldots \ldots \ldots . . . . . . . . . . .114$

Figure 46. FRF from impact at location 15 and measurement at location 1 .......................... 115

Figure 47. FRF from impact at location 15 and measurement at location 2 ......................... 115

Figure 48. FRF from impact at location 15 and measurement at location 3 ........................ 116

Figure 49. FRF from impact at location 15 and measurement at location 4 ......................... 116

Figure 50. FRF from impact at location 15 and measurement at location $5 \ldots \ldots \ldots \ldots \ldots \ldots \ldots \ldots \ldots . . . . . . . . . . . .117$

Figure 51. FRF from impact at location 15 and measurement at location 6........................ 117

Figure 52. FRF from impact at location 15 and measurement at location 7 ......................... 118

Figure 53. FRF from impact at location 15 and measurement at location 8 .......................... 118

Figure 54. FRF from impact at location 15 and measurement at location 9 .......................... 119

Figure 55. FRF from impact at location 15 and measurement at location $10 \ldots \ldots \ldots \ldots \ldots \ldots \ldots . . . . . . . . . . . . .119$

Figure 56. FRF from impact at location 15 and measurement at location $11 \ldots \ldots \ldots \ldots \ldots \ldots \ldots . . . . . . . . . . .120$

Figure 57. FRF from impact at location 15 and measurement at location 12 ........................ 120

Figure 58. FRF from impact at location 15 and measurement at location 13 ....................... 121

Figure 59. FRF from impact at location 15 and measurement at location 14 ........................ 121

Figure 60. FRF from impact at location 15 and measurement at location 15 ....................... 122 
Figure 61. FRF from impact at location 15 and measurement at location 16......................... 122

Figure 62. FRF from impact at location 15 and measurement at location 17 ........................ 123

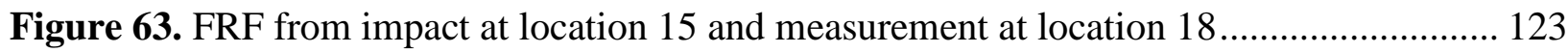




\section{INTRODUCTION}

\subsection{General}

Bridge structures may undergo various levels of vibrations during their service life. These vibrations occur due to different force producing elements at or near the surface such as traffic (human and vehicular), wind, earthquakes or other natural events. Forces such as these within the structure may lead to severe deterioration of major components of the structure or even complete collapse. A typical example of vibrations causing such a structural disaster is the Tacoma Narrows bridge collapse in 1940. The reason for collapse was the wind induced resonance, that caused the bridge to vibrate at its amplified natural frequency creating "wave" like patterns (Rogers, 1997).

Another typical example of excessive vibrations in bridges was the London Millennium Bridge that opened in June 2000 and was immediately seen to vibrate laterally on its first day. Recorded measurements showed a lateral movement of the bridge of about 2 to 3 inches. Despite there being no impending risk of structural failure, safety and comfort of pedestrians was at risk. The reason for such excessive vibration was reported to be the possibility of footfall frequency of pedestrians, correlating with the resonance frequency of the bridge. This "lock-in" phenomenon was the primary cause of the bridge being closed for further assessment and structural changes such as increased damping implemented (Dallard et al., 2001).

The probability of excessive vibrations in pedestrian bridges is high and makes them susceptible to serviceability issues more so than structural integrity ones. It is the concept of "human comfort" that is of prime importance in evaluating these bridges for serviceability. The Commonwealth of Virginia contains nearly 21,000 bridge structures in its inventory of which around 220 of those are pedestrian overpasses over highways or footbridges carrying pedestrians over non-highways. The operational safety of this vast infrastructure is directly undertaken by the Virginia Department of Transportation. Moreover, it is quite likely that pedestrian bridges are not even evaluated as consistently as other highway bridges. It is also quite possible that a large amount of pedestrian bridges, is not even included in the inventory. These structures are only rated for capacity, several of which have a multitude of details missing in the inventory (Harris et al., 2015) The capacity ratings however, do not shed light upon the serviceability of the structure or any indication of its dynamic characteristics. Due to the increasing concern of excessive vibrations on these bridges, it is imperative to understand their dynamic characteristics and document them. 


\subsection{Objectives of Thesis}

This thesis describes an experimental and analytical dynamic evaluation of a pedestrian bridge located over a highly trafficked state highway. The main goal of this thesis is to report and investigate the dynamic characteristics of an in-service pedestrian bridge and assess the structure for its serviceability based on the guidelines provided in various design standards. Several European as well as a few American standards exist that dictate the vibration serviceability assessment of bridges specifically (HIVOSS, 2007; Murray et al., 2001; SÉTRA, 2006). The thesis will provide an in-depth study of a footbridge serviceability assessment under different pedestrian loading conditions and compare the results under existing guidelines. The main topics of discussion include:

- Experimental and operational modal analysis

- Complete experimental and numerical dynamic characterization of the footbridge

- Comparative discussion of the HIVOSS, SÉTRA and AISC guidelines

The main aim of the complete project would therefore be to study how dynamic loads induced by pedestrians create comfort issues and its effect on footbridge design and maintenance.

\subsection{Organization of Thesis}

This thesis is organized into the following sections:

Chapter 1 presents description of the research problem and scope of the research. It gives a brief insight into the motivation and general purpose of conducting this research.

Chapter 2 provides the theoretical/mathematical background on the topic of vibration testing and modal analysis. The chapter is further divided into three different modal analysis methods namely theoretical, experimental and operational.

Chapter 3 discusses the existing guidelines for vibration serviceability assessment of pedestrian bridges. American guidelines of AISC and LRFD and European guidelines of HIVOSS and SÉTRA are discussed in this section.

Chapter 4 provides a further investigation and a literature review on the research topics of vibration testing and modal analysis as applied to standard bridge structures. It then presents some 
cases where this vibration evaluation was performed specifically on pedestrian bridges for serviceability assessment.

Chapter 5 presents the investigation procedure, the detailed description of the bridge and the test methods and approaches used including a preliminary finite element model followed by the experimental test setups.

Chapter 6 presents the obtained results and its following discussions in the light of the presented guidelines. Results include the obtained natural frequencies and mode shapes of the bridge which are in turn evaluated under each of the serviceability standards.

Chapter 7 summarizes the findings of the current study.

Chapter 8 suggests potential research of the topic in the future. 


\section{MODAL ANALYSIS}

Modal analysis is the tool that characterizes the dynamic properties of a structure under given theoretical or experimental conditions. Hence, through this analysis modal parameters of a structure namely the natural frequencies, mode shapes and damping ratios can be estimated (Maia \& Silva, 2014). Modal analysis can be further divided into three branches:

Theoretical Modal Analysis: This method is essentially an eigenvalue problem where the structural matrices, namely the stiffness matrix $\mathrm{K}$, mass matrix $\mathrm{M}$ and the damping matrix $\mathrm{C}$ are known. These structural matrices are then resolved to obtain the system modal parameters

Experimental Modal Analysis: This method as the name suggests is based on obtaining the modal parameters by exciting the structure using a known source of force and recording the structural response from accelerometers placed strategically on the structure. Excitations and their acceleration responses are then further processed to obtain the modal parameters.

Operational Modal Analysis: This is similar to experimental modal analysis, however the structure is not excited using a known source of force. Instead, the structure is excited under ambient conditions, the responses are measured from accelerometers on the structure and the modal parameters are then obtained by processing response only data.

\subsection{Theoretical Modal Analysis}

In order to understand the modal analysis approach used in this document a brief mathematical explanation of the classical theoretical modal analysis technique is given. The free vibration response of an $N$ degree of freedom undamped system is governed by the equation (Chopra, 2012):

$$
\mathbf{m} \ddot{\mathbf{u}}(t)+\mathbf{k} \mathbf{u}(t)=0
$$

where the matrices $\mathbf{m}$ is the mass matrix and $\mathbf{k}$ is the stiffness matrix and are defined as:

$$
\mathbf{m}=\left[\begin{array}{cccc}
m_{11} & m_{12} & \cdots & m_{1 N} \\
m_{21} & m_{22} & \cdots & m_{2 N} \\
\vdots & \vdots & \vdots & \vdots \\
m_{N 1} & m_{N 2} & \cdots & m_{N N}
\end{array}\right] \quad \text { and } \quad \mathbf{k}=\left[\begin{array}{cccc}
k_{11} & k_{12} & \cdots & k_{1 N} \\
k_{21} & k_{22} & \cdots & k_{2 N} \\
\vdots & \vdots & \vdots & \vdots \\
k_{N 1} & k_{N 2} & \cdots & k_{N N}
\end{array}\right]
$$


In the case of free vibration, for each degree of freedom present within the structure there exists a characteristic deflected shape or the "natural mode of vibration." This characteristic mode can be described mathematically as:

$$
\mathbf{u}(t)=\phi_{n} q_{n}(t)
$$

where the deflected shape $\phi_{n}$ does not vary with time. The time variation of the displacement is described by the simple harmonic function;

$$
q_{n}(t)=A_{n} \cos \left(\omega_{n} t\right)+B_{n} \sin \left(\omega_{n} t\right)
$$

where $A_{n}$ and $B_{n}$ are constants that are determined from initial conditions of motion and $\omega_{n}$ is the natural frequency. Substituting the equation (3) into (2) gives:

$$
\mathbf{k} \phi_{n}=\omega_{n}^{2} \mathbf{m} \phi_{n}
$$

The above is called the matrix eigenvalue problem. The above equation can be further simplified and solved for non-trivial solutions such that:

$$
\operatorname{det}\left(\mathbf{k}-\omega_{n}^{2} \mathbf{m}\right)=0
$$

The $N$ roots of $\omega_{n}^{2}$ determine the $N$ natural frequencies of vibration from smallest to largest. These roots are known as the eigenvalues. Once these $\omega_{n}$ are known, the corresponding vector $\phi_{n}$ can be solved. These $N$ independent $\phi_{n}$ are known as natural modes of vibration. Writing the equation for an $N$ DOF system in modal matrix form:

$$
\mathbf{k} \Phi=\mathbf{m} \Phi \mathbf{\Omega}^{2}
$$

where the matrices $\boldsymbol{\Phi}$ and $\mathbf{\Omega}^{\mathbf{2}}$ are defined as:

$$
\boldsymbol{\Phi}=\left[\begin{array}{cccc}
\phi_{11} & \phi_{12} & \cdots & \phi_{1 N} \\
\phi_{21} & \phi_{22} & \cdots & \phi_{2 N} \\
\vdots & \vdots & \vdots & \vdots \\
\phi_{N 1} & \phi_{N 2} & \cdots & \phi_{N N}
\end{array}\right] \quad \text { and } \quad \boldsymbol{\Omega}^{2}=\left[\begin{array}{cccc}
\omega_{1}{ }^{2} & & & \\
& \omega_{2}{ }^{2} & & \\
& & \ddots & \\
& & & \omega_{N}{ }^{2}
\end{array}\right]
$$

The above solution is the free vibration solution giving real values as damping was ignored. For the case of a damped system we can expand our initial equation of motion to:

$$
\mathbf{m} \ddot{\mathbf{u}}(t)+\mathbf{c} \dot{\mathbf{u}}(t)+\mathbf{k} \mathbf{u}(t)=0
$$


This can be written in terms of the natural modes of the associated undamped system using the modal expansion in equation (3):

$$
\mathbf{m} \Phi \ddot{\mathbf{q}}+\mathbf{c} \boldsymbol{\Phi} \dot{\mathbf{q}}+\mathbf{k} \boldsymbol{\Phi} \mathbf{q}=0
$$

Pre-multiplying with $\boldsymbol{\Phi}^{\mathbf{T}}$ gives:

$$
\mathbf{M} \ddot{\mathbf{q}}+\mathbf{C} \dot{\mathbf{q}}+\mathbf{K} \mathbf{q}=0
$$

Due to orthogonality matrices, $\mathbf{M}$ and $\mathbf{K}$ are diagonal. Assuming classical damping that is an uncoupled $\mathbf{C}$ matrix it will result in $N$ uncoupled differential equations in the modal coordinates $q_{n}$. The SDOF damping ratio can be obtained as follows:

$$
\zeta_{n}=\frac{C_{n}}{2 M_{n} \omega_{n}}
$$

Equation (2) can be solved to get the following displacement response:

$$
\mathbf{u}(t)=\sum_{n=1}^{N} \phi_{n} e^{-\zeta_{n} \omega_{n}}\left[\dot{q}_{n}(0) \cos \left(\omega_{d} t\right)+\frac{\dot{q}_{n}(0)+\zeta_{n} \omega_{n} q_{n}(0)}{\omega_{d}} \sin \left(\omega_{d} t\right)\right.
$$

where $\omega_{d}=\omega_{n} \sqrt{1-\zeta_{n}^{2}}$

\subsection{Experimental Modal Analysis}

In the case of experimental modal analysis, dynamic parameters (natural frequency, damping and mode shapes) are estimated by physically exciting the structure through a "controlled" forcing function (Mola et al., 2015). In order to understand the concept of experimental modal analysis some key terms are defined here:

\subsubsection{Modes and Operating Deflection Shapes}

In the process of experimental modal analysis, the structure is artificially excited and the “Operating Deflection Shapes" (ODS) are measured and then post processed to estimate the structural parameters of the tested structure. These operating deflection shapes are defined as any forced motion of two or more points on a structure such that a shape is created that is essentially the motion of one-point relative to all others (Schwarz \& Richardson, 1999). The operating deflecting shapes are in effect measured through vibration sensors in order to estimate the mode shapes and frequencies. These ODSs differ from the structure's mode shapes by their inherent 
dependence on an external force, whereas the mode shape is not dependent on any external force but is rather a unique characteristic of the structure based on its resonance (Richardson, 1997).

\section{Frequency Response Function}

In experimental modal analysis once the structure is excited, the data is recorded through accelerometers placed with specific orientations at various locations corresponding to a single degree of freedom on the structure. Modal parameters can be extracted from this data in either the time or frequency domain. In the frequency domain, a frequency response function (FRF) can be computed. Resonance frequencies can be estimated from the FRF by simply identifying the peaks of the plotted FRF. A frequency response function can be simply described as the ratio of the output response of the structure due to an applied force (Brandt, 2011):

$$
H(\omega)=\frac{X(\omega)}{F(\omega)}
$$

where $X(\omega)$ is the structure's frequency domain response and $F(\omega)$ is the forcing function.

The frequency response function is known differently based on the measured response. The flow chart below shows these terms where $D$ is the displacement response, $V$ is the velocity response, $A$ is the acceleration response and $\mathrm{F}$ is the forcing function (Irvine, 2000)

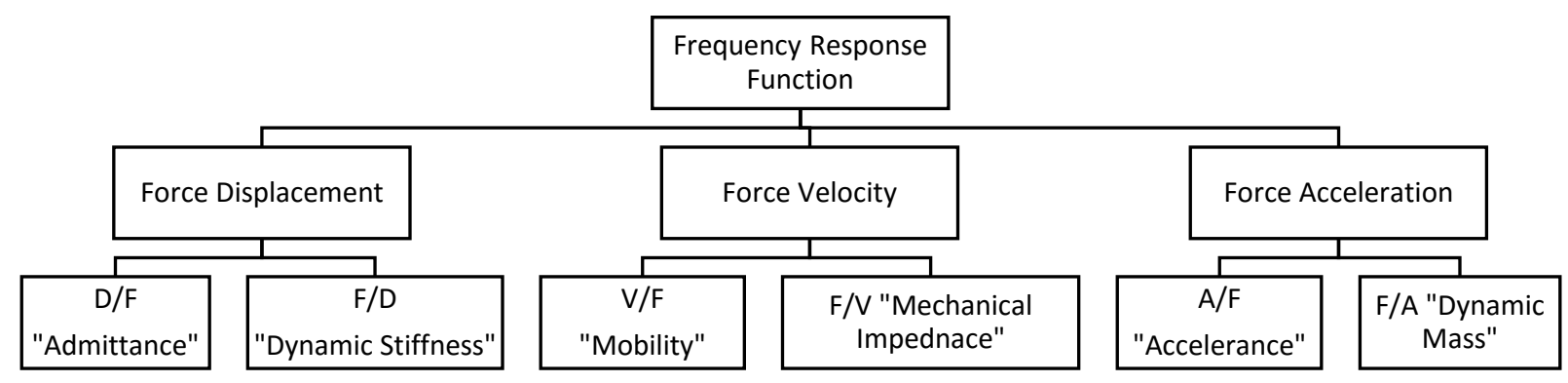

Figure 1. Frequency Response Function Types

In this document, we will focus on the Accelerance Frequency Response Function.

\section{Fourier Transform and Power Spectrums}

In physical terms, experimental data is collected as signals in the time domain. In order to convert the signal from time domain to frequency domain a Fourier Transform is required. The theory of Fourier series states that every periodic signal $x_{p}(t)$ having a time period of $T_{p}$ can be 
split into a (potentially infinite) number of sinusoidal signals each having an individual amplitude and a corresponding phase. These infinite terms can be written as a complex sum as (Brandt, 2011):

$$
x_{p}(t)=\sum_{k=-\infty}^{\infty} c_{k} e^{\frac{j 2 \pi k}{T_{p}} t}
$$

where coefficient $c_{k}$ is given by:

$$
c_{k}=\frac{1}{T_{p}} \int_{t_{1}}^{t_{1}+T_{p}} x_{p}(t) e^{-\frac{j 2 \pi k}{T_{p}} t} d t, \quad \text { for } \mathrm{k}=0,1,2, \ldots
$$

However, in the case of a bridge structure, it is assumed that vibrations on the structure are random and not periodic. These vibrations are continuous in nature and hence contain an infinite range of frequencies. Therefore, an easier way of describing this signal is through its relative likelihood of occurrence or density. The "Power Spectral Density," $S_{x x}$ of a function is hence estimated as the forward Fourier Transform of the signal's auto-correlation function $R_{x x}$ :

$$
S_{x x}(f)=\mathcal{F}\left[R_{x x}(\tau)\right]=\int_{-\infty}^{\infty} R_{x x}(\tau) e^{-j 2 \pi f \tau} d \tau
$$

where the auto-correlation function is defined as the expected value as:

$$
R_{x x}(\tau)=\operatorname{Exp}[x(t) x(t-\tau)]
$$

Similar to the power spectral density we can also define the double sided "Cross Power Spectral Density" $S_{x y}$ as the forward Fourier Transform of the cross-correlation of the input signal $x(t)$ producing an output signal $y(t)$ :

$$
S_{x y}(f)=\mathcal{F}\left[R_{x y}(\tau)\right]=\int_{-\infty}^{\infty} R_{x y}(\tau) e^{-j 2 \pi f \tau} d \tau
$$

where the cross-correlation function is defined as the expected value as:

$$
R_{x y}(\tau)=\operatorname{Exp}[x(t) y(t-\tau)]
$$

In the above relations the power spectral densities estimated are double-sided, meaning that the Fourier Transform of the signal causes half of the physical frequency content to appear at negative and the other half in the positive frequencies. Therefore, these spectral densities are then further described for just one-side as: 


$$
\begin{aligned}
& G_{x x}(f)=2 S_{x x}(f) \text { for } f>0 \\
& G_{x y}(f)=2 S_{x y}(f) \text { for } f>0
\end{aligned}
$$

\section{Welch's Method}

Building on the theoretical understanding of the spectral densities, a common method used to estimate the power spectral density and the cross power spectral density is called the Welch's method. In this method the collected signal $x(n)$ is divided into $M$ segments, each of length $N$ which are then applied a window $w$ before a Discrete Fourier Transform is obtained. This is mathematically showed as:

$$
G_{x x}(k)=\frac{S_{p}}{M} \sum_{m=1}^{M} X_{w, m}^{*} X_{w, m}, k=1,2 \ldots \ldots N / 2
$$

where $S_{p}$ is some scaling factor and $X_{w, m}$ is the discrete Fourier Transform. One segment of this discrete transform is given as:

$$
X_{w, m}(k)=\sum_{n=0}^{N-1} x_{m}(n) w(n) e^{-\frac{j 2 \pi k n}{N}}
$$

\subsubsection{FRF Estimators}

Following up from equation (12) an efficient experimentally obtained frequency response estimator would be one that minimizes the estimation error. To better understand this we can rewrite equation (12) (Rocklin et al., 1985)

$$
X(\omega)=H(\omega) F(\omega)+\varepsilon
$$

where $\varepsilon$ is the is the error vector.

The above can be rewritten in spectra forms. The first FRF assumption is that the error is incoherent with the forcing function and the second assumption is that the error is incoherent with the responses. Hence:

$$
H_{1}(\omega)=\frac{G_{x f}(\omega)}{G_{f f}(\omega)} \quad H_{2}(\omega)=\frac{G_{f x}(\omega)}{G_{x x}(\omega)}
$$

In the equations above $H_{1}$ is modelled such that Gaussian noise is added to the responses and $H_{2}$ is modelled such that Gaussian noise is added to the forcing function or input. 


\subsubsection{Modal Parameter Extraction}

There are several ways to identify modal parameters from the obtained FRFs. The most common method is the peak amplitude method whereby individual resonance peaks on the FRF are identified as the natural frequency of that mode. The FRF in this case can be written as:

$$
H(\omega)=\frac{A_{r}}{\omega_{r}^{2}-\omega^{2}+2 i \zeta_{r} \omega \omega_{r}}
$$

The width of the resonance corresponds to damping. It is estimated from the imaginary part of the complex mobility FRF using the half power bandwidth method as follows:

$$
2 \zeta_{r}=\frac{\omega_{a}^{2}-\omega_{b}^{2}}{\omega_{r}^{2}}
$$

The peaks of the imaginary part of the FRF then correspond to the mode shape:

$$
A_{r}=2|H(\omega)| \omega_{r}^{2} \zeta
$$

where $A_{r}$ is the modal constant at resonance, $\omega_{r}$ is the resonance frequency that corresponds to the natural frequency, $\omega_{a}$ and $\omega_{b}$ are the half power points at $\frac{|H(\omega)|}{\sqrt{2}}, \zeta$ is the damping ratio.

This method can be experimentally seen from Figure 2 below (Schwarz \& Richardson, 1999) :

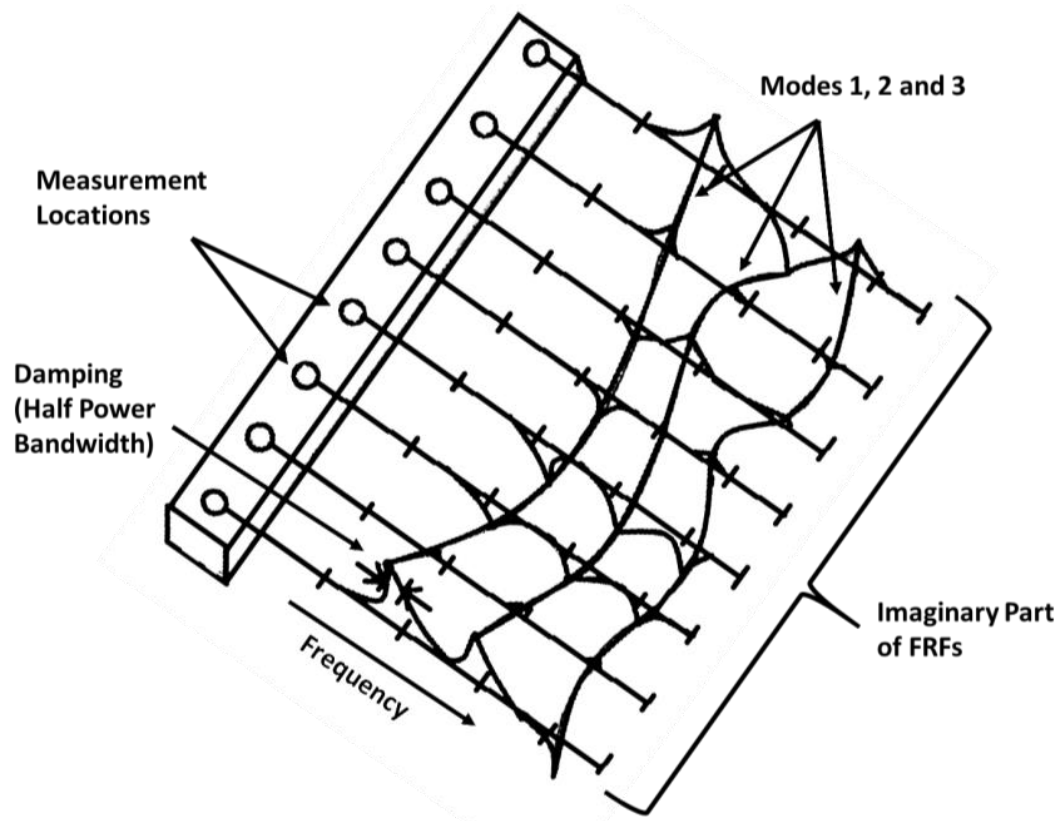

Figure 2. SDOF Peak Amplitude Method (Richardson, 1997) 


\subsubsection{Other Processing Considerations}

Following from equation (22) we can estimate a coherence function $\gamma_{x f}$ between both the FRFs as:

$$
\gamma_{x f}^{2}(\omega)=\frac{H_{1}(\omega)}{H_{2}(\omega)}=\frac{\left|G_{x f}(\omega)\right|}{G_{f f}(\omega) G_{x x}(\omega)}
$$

This is just a quality measure of the estimated frequency response regardless of which FRF estimator we actually use. It ranges between 0 to 1 where 0 is least coherence and 1 is completely coherent. In impact excitations, it is imperative to consider three additional items:

1) Pre-Trigger: Due to the inherent damping of the structure, a fast and short impulse force causes the response to exist for a short period of time. In order to ensure that both the excitation and the response signal are captured, the sampling rate is planned such that data collection starts or pre-triggers before the actual impact.

2) Windowing: Due to the signal-to-noise ratio effect or "leakage, inherent in decaying impact responses, data is windowed such as to reduce these effects. Windowing forces the signal to be periodic thereby reducing leakage effects.

3) Averaging: Since accurate impacting depends on the person impacting the structure, it is preferred to obtain multiple impacts and then average the data.

\subsection{Operational Modal Analysis}

In operational modal analysis the input source is unknown or immeasurable. The excitation is produced through ambient sources such as wind and other ground vibrations. There are a number of methods developed to obtain modal parameters using output only measurements such as Covariance-Driven Stochastic Subspace Identification (SSI-COV), Ibrahim Time Domain (ITD) and Least-Squares Complex Exponential (LSCE) (Alvaro Cunha \& Caetano, 2005). The method selected in this study for evaluating the modal parameters is Frequency Domain Decomposition (FDD). This method is very popular in civil engineering applications and estimates the modal parameters without knowing the excitation input. It is also closely related to the classical peak amplitude method in which the frequencies are peak picked. It also offers good approximation of closely spaced modes despite noise contamination. The FDD technique is explained by (Brincker et al., 2000) in detail below. 


\subsubsection{Theoretical Background}

The unknown inputs $x(t)$ and the acquired outputs $y(t)$ can be related through their power spectral densities and frequency response functions as follows:

$$
G_{y y}(j \omega)=\bar{H}(j \omega) G_{x x}(j \omega) H^{T}(j \omega)
$$

where $G_{y y}(j \omega)$ is the $(r x r)$ power spectral density (PSD) matrix of $r$ inputs and $G_{x x}(j \omega)$ is the $(m \times m)$ power spectral density matrix of m outputs. $H(j \omega)$ is the $(m \times r)$ frequency response function (FRF) matrix with ${ }^{-}$and ${ }^{\mathrm{T}}$ denoting the complex conjugate and transpose respectively.

The partial fraction expansion of the FRF can be written in terms of pole/residue as follows:

$$
H(j \omega)=\sum_{k=1}^{n} \frac{R_{k}}{j \omega-\lambda_{k}}+\frac{\overline{R_{k}}}{j \omega-\overline{\lambda_{k}}}
$$

where $n$ is the number of modes $\lambda_{k}$ is the pole and $R_{k}$ is the residue. If the input is assumed to be white noise, then the PSD becomes a constant matrix and equation (27) can be rewritten as:

$$
G_{y y}(j \omega)=\sum_{k=1}^{n} \sum_{s=1}^{n}\left[\frac{R_{k}}{j \omega-\lambda_{k}}+\frac{\overline{R_{k}}}{j \omega-\overline{\lambda_{k}}}\right](C)\left[\frac{R_{s}}{j \omega-\lambda_{s}}+\frac{\overline{R_{s}}}{j \omega-\overline{\lambda_{s}}}\right]^{H}
$$

where superscript $H$ denotes the complex conjugate. The above expression is then reduced to a pole/residue form using partial fractions as follows:

$$
G_{y y}(j \omega)=\sum_{k=1}^{n} \frac{A_{k}}{j \omega-\lambda_{k}}+\frac{\overline{A_{k}}}{j \omega-\overline{\lambda_{k}}}+\frac{B_{k}}{-j \omega-\lambda_{k}}+\frac{\overline{B_{k}}}{-j \omega-\overline{\lambda_{k}}}
$$

where $A_{k}$ is the $k$ 'th residue matrix of the output PSD which is also an ( $m \times m$ ) Hermitian matrix:

$$
A_{k}=R_{k} C\left(\sum_{s=1}^{n} \frac{\overline{R_{s}^{T}}}{-\lambda_{k}-\bar{\lambda}_{s}}+\frac{R_{s}^{T}}{-\lambda_{k}-\lambda_{s}}\right)
$$

The contribution from the $k$ th mode is given by:

$$
A_{k}=\frac{R_{k} C \overline{R_{k}^{T}}}{2 \alpha_{k}}
$$


where $\alpha_{k}$ is minus the real part of the pole $\lambda_{k}=-\alpha_{k}+j \omega_{k}$. Under light damping the residue becomes proportional to the mode shape vector:

$$
A_{k} \propto R_{k} C \overline{R_{k}^{T}}=\phi_{k} \gamma_{k}^{T} C \gamma_{k} \phi_{k}^{T}=d_{k} \phi_{k} \phi_{k}^{T}
$$

where $d_{k}$ is a scalar constant. At a certain frequency only a small number of modes will contribute significantly, typically one or two. Denoting these by $\operatorname{Sub}(\omega)$, then a lightly damped structure's output spectral density would be written as:

$$
G_{y y}(j \omega)=\sum_{k \in S u b(\omega)} \frac{d_{k} \phi_{k} \phi_{k}^{T}}{j \omega-\lambda_{k}}+\frac{\overline{d_{k}} \overline{\phi_{k}} \overline{\phi_{k}^{T}}}{j \omega-\overline{\lambda_{k}}}
$$

\subsubsection{The FDD Algorithm}

The first step in the process is to estimate the power spectral density matrix $G_{y y}(j \omega)$ for the outputs from the experimental data. The output PSD known at discrete frequencies $\omega=\omega_{i}$ is then decomposed by taking the Singular Value Decomposition (SVD) of the matrix;

$$
G_{y y}\left(j \omega_{i}\right)=U_{i} S_{i} U_{i}^{H}
$$

where the matrix $U_{i}=\left[u_{i 1}, u_{i 2}, \ldots, u_{i m}\right]$ is the unitary matrix holding singular vectors $u_{i i}$ and $S_{i}$ is a diagonal matrix holding the scalar singular values $s_{i i}$. At a peak corresponding to the $k$ 'th mode in the spectrum this mode or a close mode will be dominating. If only the $k$ 'th mode is dominating, there will only be one term in equation (34). The first singular vector in this case $u_{i 1}$ would be an estimate of the mode shape:

$$
\phi=U_{i 1}
$$

The corresponding singular value is the auto power spectral density function of the single degree of freedom system. This power spectral density function is identified by isolating the peak and comparing the mode shape estimate with the singular vectors obtained for frequency lines around the peak. The peaks selected from the singular values plot will correspond to the natural frequency of the system.

\subsubsection{The EFDD Extension}

Following the above algorithm, an extension of FDD was further proposed (Brincker et al., 2001) to estimate damping known as the Enhanced Frequency Domain Decomposition. The 
obtained auto power spectral density function that is $S_{i 1}$ is converted from frequency domain back to time domain using the inverse Fourier Transform. This time domain function is also the auto correlation function of the single degree of freedom system. From this time domain function, all extreme values $r_{k}$ (including both peaks and valleys) are obtained. The logarithmic decrement is then estimated as:

$$
\delta=\frac{2}{k} \ln \left(\frac{r_{0}}{\left|r_{k}\right|}\right)
$$

where $r_{0}$ is the initial value of the correlation function and $r_{k}$ is the $k$ 'th extreme. The damping ratio is then given by:

$$
\zeta=\frac{\delta}{\sqrt{\delta^{2}+4 \pi^{2}}}
$$

The natural frequency in this case however is then estimated by performing a linear regression between the crossing times and the times corresponding to the peaks. Therefore, the natural frequency would then be estimated as:

$$
\omega=\frac{\omega_{d}}{\sqrt{1+\xi^{2}}}
$$

Similar to equation (26), in order to assess the correlation between one mode and another such as comparing analytical $\left\{\phi_{A}\right\}_{r}$ and experimental modes $\left\{\phi_{X}\right\}_{q}$, a statistical indicator known as Modal Assurance Criteria can be estimated using the relative modal vectors as follows:

$$
M A C=\frac{\left|\left\{\phi_{A}\right\}_{r}^{T}\left\{\phi_{X}\right\}_{q}\right|^{2}}{\left(\left\{\phi_{A}\right\}_{r}^{T}\left\{\phi_{A}\right\}_{r}\right)\left(\left\{\phi_{X}\right\}_{q}^{T}\left\{\phi_{X}\right\}_{q}\right)}
$$




\section{DESIGN GUIDELINES FOR SERVICEABILITY ASSESSMENT}

Civil structures such as bridges undergo regular vibrations during their normal operation. These vibrations are caused mainly by dynamic loads such as traffic or other ambient conditions such as wind. A unique case of these vibrations is presented in pedestrian bridges. Inherent to their characteristics, pedestrian bridges are usually lighter and contain slenderer members owing to lower loading demands. The structural properties of these structures tend to be on a much lower side causing relatively lower natural frequency and stiffness ranges. These bridges then become easily susceptible to vibrations from pedestrian traffic or "human induced vibrations." (Gheitasi et al., 2016). This phenomenon leads to the safety and stability of these structures or serviceability assessment. The most well documented case in this topic has been the London Millennium Bridge (Dallard et al., 2001). There are two major checks for which the serviceability assessment is conducted. These are the natural frequency limits and the maximum acceleration limits. Several design codes exist that specify frequency and acceleration limits of pedestrian bridges under traffic.

The most commonly used codes will be discussed in the following sections. For a general understanding of the serviceability assessment process a flowchart is provided below (Grandić, 2015) 


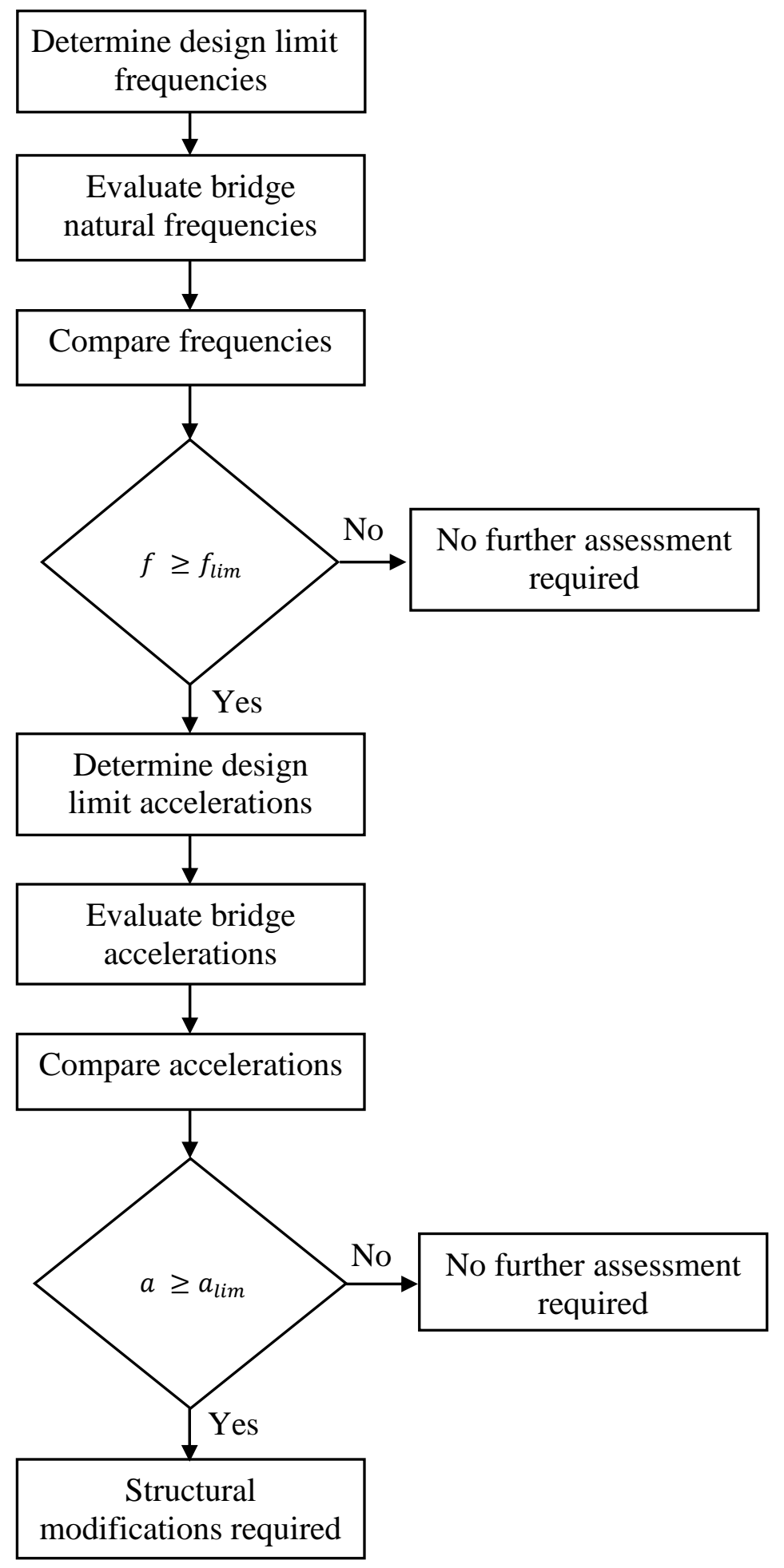

Figure 3. Serviceability Assessment Flowchart (Grandić, 2015) 


\subsection{Pedestrian Loading}

Just like any other forced vibration system, a pedestrian bridge undergoes a dynamic response under an input force, in this case this force is caused by the pedestrian loading. Due to the various different types of moving activities such as walking and running, performed by pedestrians on a bridge, the nature of pedestrian loading is fairly complex. Several models exist in the literature that theoretically estimate the loading caused by a pedestrian/s walking on a bridge (Hauksson, 2005; S. Živanović et al., 2005)

Assuming that the forcing functions described are all periodic under the rationale that during an activity such as walking and running, each step force is repeatable with human motion and is exactly similar to the step before, we can theoretically predict these forces using the Fourier series (Heinemeyer et al., 2009):

$$
\begin{aligned}
& F_{p, v e r t}(t)=P\left[1+\sum_{i=1}^{n} \alpha_{i, v e r t} \sin \left(2 \pi j f_{s} t-\phi_{i}\right)\right] \\
& F_{p, \text { lat }}(t)=P \sum_{i=1}^{n} \alpha_{i, \text { lat }} \sin \left(\pi j f_{s} t-\phi_{i}\right)
\end{aligned}
$$

where $F_{p, v e r t}(t)$ and $F_{p, l a t}(t)$ are the vertical and lateral periodic forces due to human walking or running, $\mathrm{P}$ is the static load corresponding to the pedestrian's weight, $f_{s}$ is the step frequency, $\alpha_{i, v e r t}$ and $\alpha_{i, l a t}$ are the Fourier coefficients of the $i$ th harmonic for vertical and lateral forces. These coefficients are also known as Dynamic Load Factors (DLFs). Several authors have reported different values for the dynamic load factors. For example, one model proposes a vertical DLF of 0.257 in the case of the first harmonic from walking. This dynamic load factor is then decreased as frequency of walking is increased. The overall frequencies of the common human activities on a pedestrian bridge can be estimated as shown in Table 1 (S. Živanović et al., 2005):

Table 1. Frequencies of common human activities

\begin{tabular}{lccc}
\hline & Slow $(\mathbf{H z})$ & Normal & Fast \\
\hline Walking & $1.4-1.7$ & $1.7-2.2$ & $2.2-2.4$ \\
Running & $1.9-2.2$ & $2.2-2.7$ & $2.7-3.3$ \\
Jumping & $1.3-1.9$ & $1.9-3.0$ & $3.0-3.4$ \\
\hline
\end{tabular}

The above table gives an idea of the average human motion frequencies. These can also be compared to the codified frequency assessment limits of the walking structure. 
As discussed earlier, several codified guidelines exist for the design and assessment of dynamic response from pedestrian bridges. Table 2 shows the frequency limits imposed by some of these codes under which a further assessment must be carried out (Gheitasi et al., 2016):

Table 2. Range of Frequencies by Design Codes (AASHTO, 2009; HIVOSS, 2007a; SÉTRA, 2006)

\begin{tabular}{lcc}
\hline \multicolumn{1}{c}{ Reference } & Vertical & Transverse \\
\hline British National Annex & $1.5-5 \mathrm{~Hz}$ & $<1.5 \mathrm{~Hz}$ \\
Eurocode 1990 & $<5 \mathrm{~Hz}$ & $<2.5 \mathrm{~Hz}$ \\
SÉTRA & $1-5 \mathrm{~Hz}$ & $0.3-2.5 \mathrm{~Hz}$ \\
HIVOSS & $1.25-4.6 \mathrm{~Hz}$ & $0.5-1.2 \mathrm{~Hz}$ \\
AASHTO LRFD & $<3 \mathrm{~Hz}$ & $<1.3 \mathrm{~Hz}$ \\
\hline
\end{tabular}

The table above is only a brief indicator of the range of frequencies under which each of the codes will carry out a further evaluation. However, not every code has strict frequency restrictions or follows the exact same criteria as shown in the flowchart above. Some of the most common design codes are discussed in detail here:

\subsection{AASHTO LRFD Guide Specifications for the Design of Pedestrian Bridges (NCHRP 20-07 TASK 244)}

This is an additional guidance for the design and construction of pedestrian bridges in supplement to the AASHTO LRFD Bridge Design Specifications. These guide specifications are not specific to vibration serviceability; however, they briefly define certain limits. The specifications define the limit of the vertical natural frequency to be no less than $3 \mathrm{~Hz}$ in order to avoid the first harmonic from walking. This limit in the lateral direction is set at $1.3 \mathrm{~Hz}$. If these limits are not met, then a secondary evaluation is to be made. Table 3 shows this criteria (AASHTO, 2009):

Table 3. AASHTO LRFD Frequency Limit

\begin{tabular}{llc}
\hline & Vertical & Lateral \\
\hline Frequency Limit & $>3 \mathrm{~Hz}$ & $>1.3 \mathrm{~Hz}$ \\
\hline
\end{tabular}

This evaluation will have to take into account the frequency and magnitude of pedestrian footfall loadings, the phasing of this loading from multiple pedestrians including the "lock-in 
phenomena," the structural damping estimation and the acceleration limits. To account for all the above items, the specifications alternatively suggest the evaluation of the following criteria:

$$
f \geq 2.86 \ln \left(\frac{180}{W}\right) \quad \text { or } \quad W \geq 180 e^{-0.35 f}
$$

where $W$ is the weight of the support structure including dead load and $f$ is the fundamental frequency in the vertical direction.

\subsection{AISC Steel Design Guide No. 11 (Floor Vibrations due to Human Activity)}

This criterion is based on the dynamic response of steel beam or joist supported floor systems to walking forces and can be extended to incorporate footbridges as well. The guideline for human comfort design is based on three different components. Firstly, the guidelines suggest acceptable acceleration limits as a percentage of peak acceleration over the acceleration of gravity. These values differ from one activity to another. In terms of comfort, people usually have lower acceleration acceptance limits for vibration frequencies between $4-8 \mathrm{~Hz}$. The recommended peak acceleration limits for different occupancies can be seen from Figure 4 (Murray et al., 2003):

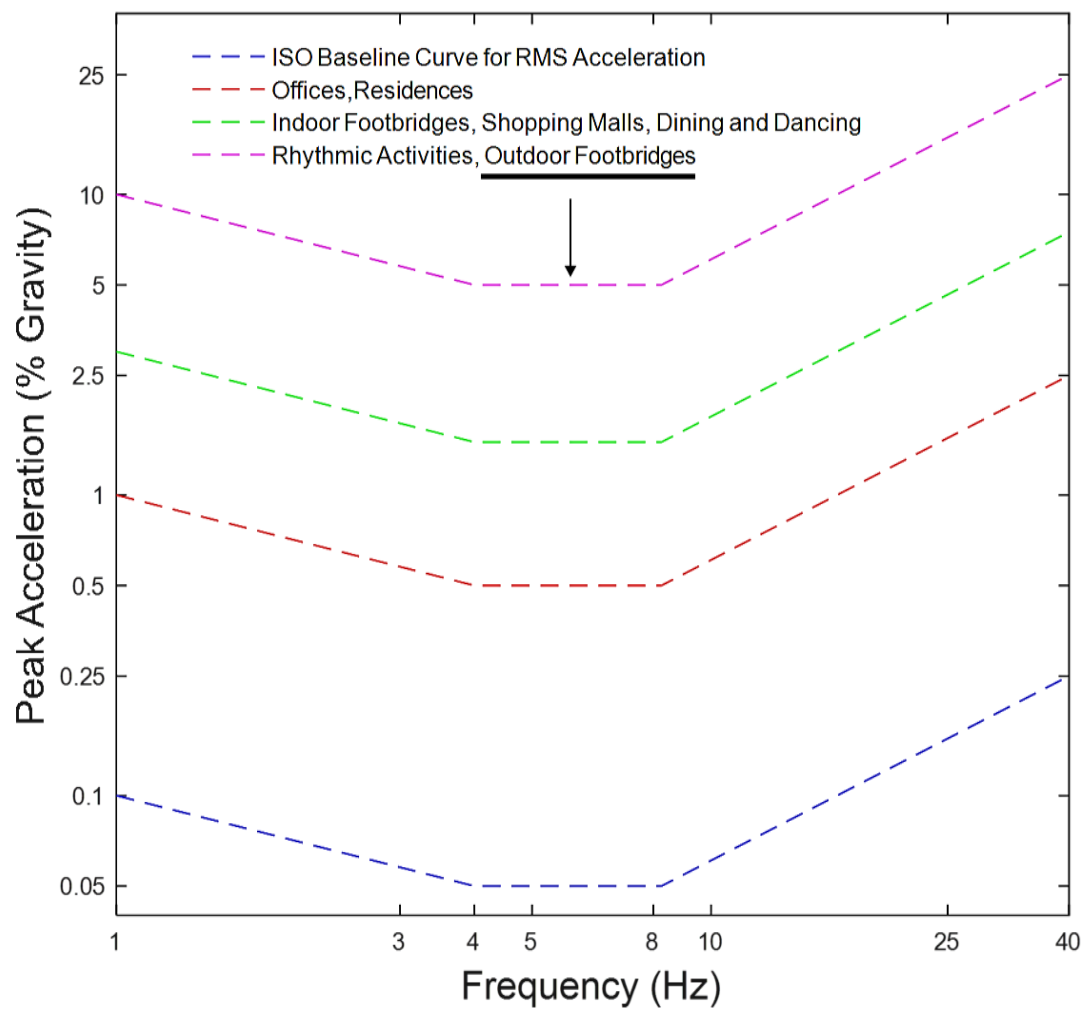

Figure 4. International Standardization Organization (ISO) Peak Acceleration Limits (Murray et al., 2003) 
The second item for the design criteria are the recommended values of the Fourier coefficients in the pedestrian harmonic load model from equation (41-42). Several researchers have recommended coefficients that differ from case to case (Bachmann \& Ammann, 1987; Blanchard et al., 1977; Yao et al., 2002). In the AISC Steel Design guide, the recommended values for the Fourier coefficients for a person walking, are as follows:

Table 4. Recommended Fourier Coefficients for Loading Model

\begin{tabular}{ccc}
\hline \multirow{2}{*}{ Harmonic, $\boldsymbol{i}$} & \multicolumn{2}{c}{ Person Walking } \\
\cline { 2 - 3 } & $\boldsymbol{F , H z}$ & $\boldsymbol{\alpha}_{\mathbf{i}}$ \\
\hline 1 & $1.6-2.2$ & 0.5 \\
2 & $3.2-4.4$ & 0.2 \\
3 & $4.8-6.6$ & 0.1 \\
4 & $6.4-8.8$ & 0.05 \\
\hline
\end{tabular}

The third part of the design criteria takes into account the resonance response function which essentially is just the ratio of the floor acceleration to the acceleration of gravity and is given as:

$$
\frac{a_{p}}{g}=\frac{P_{o} \exp \left(-0.35 f_{n}\right)}{\beta W} \leq \frac{a_{o}}{g}
$$

Where $\frac{a_{p}}{g}$ is the estimated peak accelerations in $\mathrm{g}, \frac{a_{o}}{g}$ is the acceleration limit from the Figure $4, f_{n}$ is the natural frequency of the floor, $P_{o}$ is the constant force equal to $0.41 \mathrm{kN}$ (92 lbs) for footbridges, $\beta$ is the modal damping ratio and $W$ is the effective weight of the floor.

The formula above shows that for a fundamental frequency greater than $8 \mathrm{~Hz}$, the acceleration limit does not increase. This is different than the limit shown in the Figure 4 because in the figure, peak acceleration limits keep increasing beyond $8 \mathrm{~Hz}$. This is done to approximately account for footstep impulse vibration. For natural frequencies greater than $9-10 \mathrm{~Hz}$ an additional stiffness check is implemented:

$$
k \geq 1 \mathrm{kN} / \mathrm{mm} \text { or } k \geq 5.7 \mathrm{kips} / \mathrm{in}
$$

The standard also suggests a simplified procedure for determining the first vertical natural frequency of a simple floor or beam supported on steel girders between columns as follows:

$$
f_{n}=0.18 \sqrt{g / \Delta}
$$


where $\Delta$ is the midspan deflection of the member relative to its supports due to the loading. In a simple span assumption, this can be estimated as:

$$
\Delta=5 w L^{4} /\left(384 E_{s} I_{t}\right)
$$

where $w$ is the uniformly distributed load per unit length support by the member, $E_{s}$ is the modulus of steel, $I_{t}$ is the transformed moment of inertia and $L$ is the member span.

Figure 4 can also be used to extend the original plateau to 3-20 Hz. It is finally mentioned that floor systems with natural frequency less than $3 \mathrm{~Hz}$ should be generally avoided.

\subsection{HIVOSS (Human Induced Vibrations of Steel Structures) (HIVOSS, 2007)}

This is the European design and assessment guide of the vibration serviceability. HIVOSS identifies several step wise design criteria for footbridges. HIVOSS suggests the usage of a finite element model and experimental testing to determine the natural frequency content of the footbridge. It also defines additional revisions to the modal mass of the structure. Utilizing the pedestrian force models and their harmonic representation as defined in the previous section, HIVOSS provides the following critical range of frequencies:

$$
\begin{array}{lc}
\text { For vertical and longitudinal vibration: } & 1.25 \leq f \leq 4.6 \mathrm{~Hz} \\
\text { For lateral vibration: } & 0.5 \leq f \leq 1.2 \mathrm{~Hz}
\end{array}
$$

Therefore, footbridges that have their natural frequencies within the limits defined above must be subjected to a dynamic assessment to pedestrian excitation. For the dynamic response assessment of footbridges, the next step is to define a set of physical conditions representing real conditions that can occur during service time of the footbridge. HIVOSS defines five traffic classes based on pedestrian densities $d$ (peds. $/ \mathrm{m}^{2}$ ) as shown in Table 5 and four comfort levels, ranging from unacceptable vibration levels to maximum comfort as shown in Table 6.

Table 5. Traffic classes defined in HIVOSS

\begin{tabular}{lll}
\hline Traffic Class & Density, $\boldsymbol{d}(\boldsymbol{p}=$ pedestrian $)$ & Description \\
\hline TC 1 & $15 p /$ deck area & Very weak traffic \\
TC 2 & $0.2\left(p / m^{2}\right)$ & Weak traffic \\
TC 3 & $0.5\left(p / m^{2}\right)$ & Dense traffic \\
TC 4 & $1.0\left(p / m^{2}\right)$ & Very dense traffic \\
TC 5 & $1.5\left(p / m^{2}\right)$ & Exceptionally dense traffic \\
\hline
\end{tabular}


Table 6. Comfort levels defined in HIVOSS (*Limit value defined in SÉTRA guidelines)

\begin{tabular}{llll}
\hline Comfort level & Description & Vertical $\boldsymbol{a}_{\text {limit }}\left(\boldsymbol{m} / \boldsymbol{s}^{\mathbf{2}}\right)$ & Transverse $\boldsymbol{a}_{\text {limit }}\left(\boldsymbol{m} / \boldsymbol{s}^{\mathbf{2}}\right)$ \\
\hline CL 1 & Maximum & $<0.5$ & $<0.1\left(0.15^{*}\right)$ \\
CL 2 & Medium & $0.5-1.0$ & $0.1\left(0.15^{*}\right)-0.3$ \\
CL 3 & Minimum & $1.0-2.5$ & $0.3-0.8$ \\
CL 4 & Unacceptable & $>2.5$ & $>0.8$ \\
\hline
\end{tabular}

The guidelines also define a "lateral lock-in" phenomena, whereby a pedestrian in order to compensate for his center of gravity and lateral stability will try to move with the bridge's lateral displacement motion. This will cause the laterally moving footbridge to vibrate even further as essentially the pedestrian has caused his moving motion harmonic to coincide with that of the bridge causing a higher resonance amplitude. This phenomenon was observed to occur at a trigger amplitude of $0.1-0.15 \mathrm{~m} / \mathrm{s}^{2}$.

The next step in the HIVOSS guidelines is to calculate the maximum acceleration that the footbridge will experience under each design situation. There are three alternative methods described in the guidelines for the evaluation of acceleration. In the first two methods, that is the SDOF method and the finite element method, a harmonic load model is used that simplifies the random pedestrian load corresponding to a pedestrian density $d$ to an equivalent deterministic load uniformly distributed on the bridge deck. This equivalent number of perfectly synchronized pedestrians, $n^{\prime}$ corresponds to a stream of $n$ random pedestrians as follows:

$$
\begin{array}{ll}
n^{\prime}=10.8 \sqrt{\zeta n} & \left(\mathrm{TC} 1 \text { to TC3, } d<1.0 \mathrm{p} / \mathrm{m}^{2}\right) \\
n^{\prime}=1.85 \sqrt{n} & \left(\text { TC4 to TC5, } d \geq 1.0 \mathrm{p} / \mathrm{m}^{2}\right)
\end{array}
$$

where $\zeta$ is the structural damping ratio. Both models can be defined by a uniformly distributed harmonic load corresponding to the equivalent pedestrian stream as follows:

$$
p(t)=P \cos \left(2 \pi f_{s} t\right) \frac{n^{\prime}}{S} \psi
$$

where $P$ is the component of the force due to a single pedestrian and given as $280 \mathrm{~N}$ for vertical vibrations and $35 \mathrm{~N}$ for transverse vibrations, $\mathrm{S}$ is the area of the loaded surface (note that $n=S \times$ $d), f_{s}$ is the walking frequency of the pedestrian, and $\psi$ is the reduction coefficient that accounts for the probability that the step frequency or its second harmonic approaches the considered natural 
frequency of the footbridge. This reduction coefficient is illustrated as a function of the structure's frequency for the vertical and transverse vibrations in Figure 5 below:

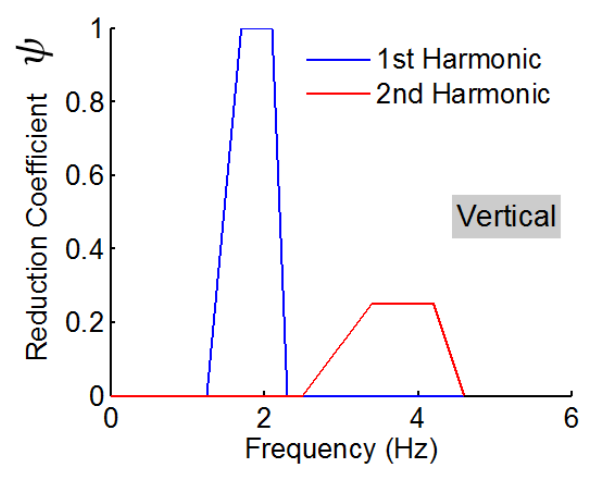

(a)

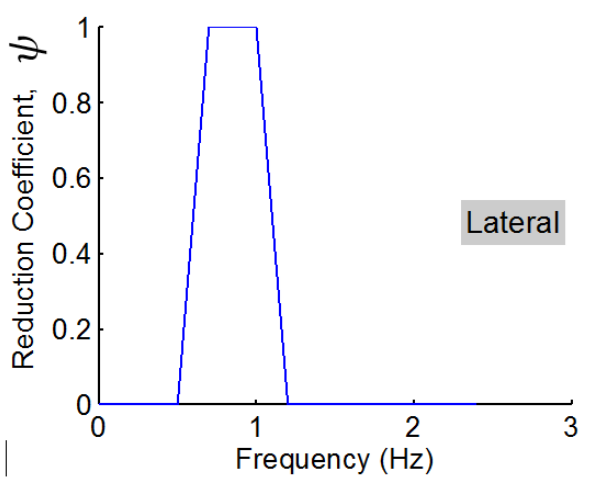

(b)

Figure 5. Reduction coefficient $\psi$ for (a) vertical and (b) transverse vibrations according to HIVOSS guidelines.

A finite element model or an equivalent SDOF system of the footbridge is then used to compute the associated maximum acceleration for given dynamic loading. If the SDOF approach is used, the maximum acceleration at resonance under the above described load model can be estimated as:

$$
a_{\max }=\frac{p^{*}}{m^{*}} \frac{1}{2 \zeta}
$$

where $p^{*}$ is the generalized load and calculated as $\frac{2 p L}{\pi}$ for a distributed load $p$ per unit length and $m^{*}$ is the generalized mass and calculated as $\frac{m_{s} L}{2}$ for given distributed mass $m_{s}$ per unit length.

The third method is a response spectra approach that aims to describe the stochastic loading and system response with a specific confidence level based on 5000 different pedestrian streams. Each of these streams had statistically random properties including pedestrian weight, step frequency, start position, moment of first step etc. The following empirical equation is provided to predict the characteristic acceleration, $95^{\text {th }}$ percentile of maximum acceleration based on different pedestrian densities:

$$
a_{\max , 95 \%}=k_{a, 95 \%} \sigma_{a}=k_{a, 95 \%} \frac{\sigma_{F}}{m_{i}^{*}} \sqrt{k_{1} C \zeta^{k_{2}}}
$$

where $k_{a, 95 \%}$ is the peak factor that transforms the standard deviation of the acceleration response to the 95 th percentile characteristic value, $\sigma_{a}^{2}$ is the variance of the acceleration response, $\sigma_{F}$ is the 
standard deviation of the pedestrian loading, $C$ is the constant describing the maximum of the load spectrum, $k_{1}$ and $k_{2}$ are constants which depend on the considered natural frequency and pedestrian density, $m_{i}^{*}$ is the modal mass of the considered mode $i$.

Therefore, the above criteria can be used to assess the serviceability of a footbridge under the European guidelines. HIVOSS also suggests further testing procedures and structural modifications that is beyond the scope of this document.

\subsection{SÉTRA (Assessment of Vibrational Behavior of Footbridges under Pedestrian Loading) (SÉTRA, 2006)}

The SÉTRA guidelines define four footbridge classes: Class I - urban footbridge with very heavy traffic; Class II - urban footbridge with heavy traffic; Class III - footbridge for standard use; and Class IV - seldom used footbridge. The comfort levels are defined in the same manner with HIVOSS guidelines as given in Table 6 except that the limiting acceleration value for maximum comfort for transverse vibrations is $0.15 \mathrm{~m} / \mathrm{s}^{2}$. For Class I to III footbridges, a dynamic analysis might be required if their natural frequency is below $5 \mathrm{~Hz}$ for vertical vibrations and below $2.5 \mathrm{~Hz}$ for transverse vibrations. Table 7 provides the load cases that need to be considered in the dynamic analysis depending on the footbridge's class and natural frequency. Case I corresponds to a loading due to sparse or dense crowds and is considered for Class II and Class III footbridges. Case II represents a very dense crowd loading and is considered only for Class I footbridges. Case III considers the second harmonic of the vibrations caused by pedestrian loads and is taken into account for Class I and II footbridges. For all loading cases, the loads for dynamic analysis are calculated using the equations (48) to (50) given above. The pedestrian density $d$ and the force due to a single pedestrian walking $P$ are given in Table 8 for each loading case. The reduction coefficient $\psi$ according to SÉTRA guidelines is given in Figure 6. The maximum acceleration of the footbridge is calculated using a SDOF approach and given as follows:

$$
a_{\max }=\frac{1}{2 \zeta} \frac{4|p(t)| w_{d}}{\rho S \pi}
$$

where $|p(t)|$ is the magnitude of the applied load $p(t) ; w_{d}$ is the available width of the deck for pedestrian walking; and $\rho S$ is the total linear density, which is calculated as the sum of the linear density of deck and that of the pedestrians. 
Table 7. Load cases to select for dynamic analysis

\begin{tabular}{|c|c|c|c|}
\hline \multirow[b]{2}{*}{ Footbridge class } & \multicolumn{3}{|c|}{ Frequency range (V: Vertical, T: Transverse) } \\
\hline & $\begin{array}{c}\text { Range 1 } \\
V=1.7-2.1 \mathrm{~Hz} \\
\mathrm{~T}=0.5-1.1 \mathrm{~Hz}\end{array}$ & $\begin{array}{c}\text { Range } 2 \\
\mathrm{~V}=\mathbf{1 . 0}-1.7 \mathrm{~Hz} \text { and } 2.1-2.6 \mathrm{~Hz} \\
\mathrm{~T}=\mathbf{0 . 3}-\mathbf{0 . 5} \mathrm{Hz} \text { and } 1.1-1.3 \mathrm{~Hz}\end{array}$ & $\begin{array}{c}\text { Range } 3 \\
V=2.6-5.0 ~ H z \\
T=1.3-2.5 ~ H z\end{array}$ \\
\hline Class I & Case 2 & Case 2 & Case 3 \\
\hline Class II & Case 1 & Case 1 & Case 3 \\
\hline Class III & Case 1 & - & - \\
\hline
\end{tabular}

Table 8. Pedestrian loads for different loading cases

\begin{tabular}{ccccccc}
\hline \multirow{2}{*}{$\begin{array}{c}\text { Loading } \\
\text { case }\end{array}$} & \multicolumn{2}{c}{ Pedestrian density, $\boldsymbol{d}\left(\boldsymbol{p} / \boldsymbol{m}^{\mathbf{2}}\right)$} & \multicolumn{3}{c}{ Single pedestrian force, $\boldsymbol{P}(\boldsymbol{N})$} \\
\cline { 2 - 7 } & Class I & Class II & Class III & Class I & Class II & Class III \\
\hline Case 1 & - & 0.8 & 0.5 & - & $\begin{array}{c}280(\mathrm{~V}) \\
35(\mathrm{~T})\end{array}$ & $\begin{array}{c}280(\mathrm{~V}) \\
35(\mathrm{~T})\end{array}$ \\
\hline Case 2 & 1.0 & - & - & $\begin{array}{c}280(\mathrm{~V}) \\
35(\mathrm{~T})\end{array}$ & - & - \\
\hline Case 3 & 1.0 & 0.8 & - & $\begin{array}{c}70(\mathrm{~V}) \\
7(\mathrm{~T})\end{array}$ & $\begin{array}{c}70(\mathrm{~V}) \\
7(\mathrm{~T})\end{array}$ & - \\
\hline
\end{tabular}

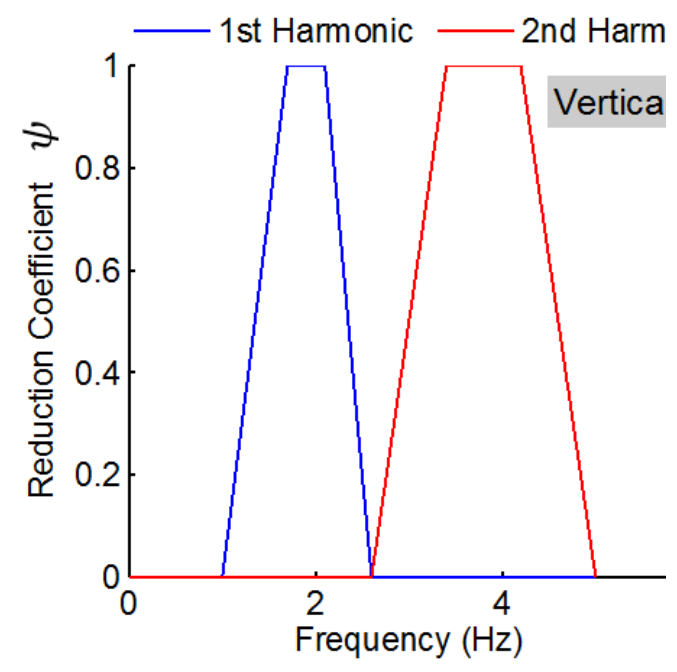

(a)

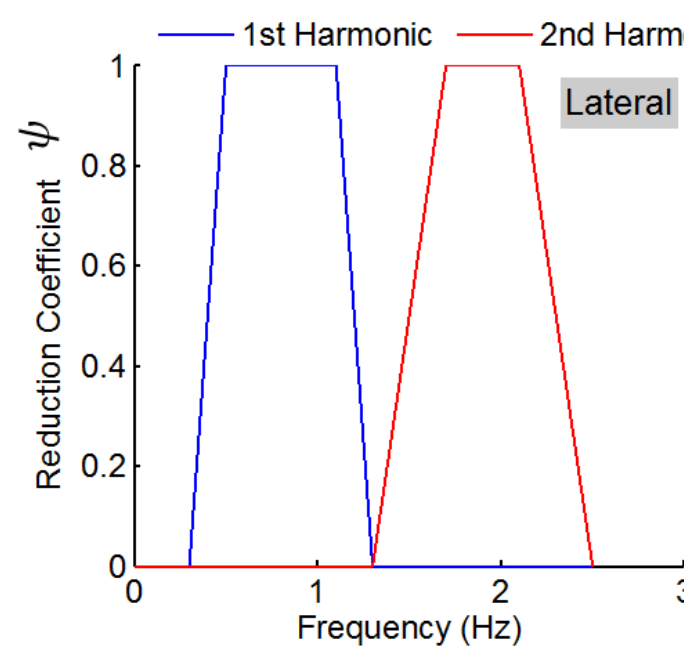

(b)

Figure 6. Reduction coefficient for (a) vertical and (b) transverse vibrations according to SÉTRA guidelines 


\subsection{General Comments}

Some comparisons can now be outlined after reviewing the guidelines mentioned in this section. The three main guidelines HIVOSS, SÉTRA and the AISC Guide 11 can be compared as follows.

- In HIVOSS and SÉTRA a traffic class and comfort level is defined based on the pedestrian density, location of the bridge and usage. These in effect then correspond to an acceleration limit. In the AISC design guide peak accelerations are graphically obtained from the ISO 2631-2 based curve.

- For the harmonic load prediction, both HIVOSS and SÉTRA provide a range of values for the reduction coefficient but the AISC design guide provides a simplified exact numerical estimate to be used for outdoor footbridges.

- An in-depth calculation for loading is provided under HIVOSS and SÉTRA but only specific load values are provided for chosen harmonics in AISC.

- HIVOSS and SÉTRA contain provisions for vertical, longitudinal and transverse motion. Therefore, they can be used to assess for frequencies and accelerations in any of the three directions. AISC is however applied for only vertical motion.

- AISC guide is a comprehensive guide for all steel structures especially floor systems and incorporates other structures such as footbridges as well. HIVOSS and SÉTRA are specifically used only for footbridges.

In general, it can be deduced that HIVOSS and SÉTRA provide an extensive mathematical background and analytical solutions whereas the AISC design guide provides only simplified solutions and estimates. Both the European guides are more extensive and provide greater detail than the AISC guide. Despite, its simplistic nature the AISC design guide fails to provide an indepth discussion on vibration serviceability specific to footbridges. 


\section{BACKGROUND RESEARCH}

Dynamic testing of bridges has been conducted on bridge structures for some time (AbdelGhaffar \& Scalan, 1985), however with modern technological advances and fast signal processing methods it has now become possible to conduct and process vibration data from bridges in real time, with better precision and accuracy. Several extensive studies exist that deal with this topic. The following literature review for this document has been divided into two sections. First an outline of the recent works pertaining to dynamic testing of bridge structures, their instrumentation plans, signal processing schemes and their resulting dynamic properties are presented. This is followed by a brief background of vibration testing of pedestrian bridges. This will include the incorporation of estimated dynamic properties and the assessment of pedestrian bridges for vibration serviceability.

\subsection{Vibration Testing and Modal Analysis}

An important aspect of bridge structures is their dynamic characteristics. The most important of which is the "natural frequency" of the structure as it inherently defines the system in terms of its mass and stiffness. This key dynamic property aids the understanding of the structure's vibration modes causing severe serviceability issues. Vibration experimental studies on bridge structures have been conducted by numerous researchers, some of who are discussed below (Cunha et al., 2012; Bačinskas et al., 2013; Brownjohn et al., 2003; Halling et al. , 2004; Rahmatalla et al., 2014; Wang et al., 2005; Whelan et al., 2009). The underlying commonality between each of these researches is their main goal of finding the structure's natural frequency and its modes of vibration. There are numerous ways in which a researcher can conduct this analysis. The following literature review provides a comprehensive background of the different testing techniques used by these researchers, their testing plans, processing techniques and the resulting dynamic properties:

Assessment of Highway Bridge Upgrading by Dynamic Testing and Finite-Element Model Updating (Brownjohn et al., 2003)

Researchers in this article conducted an extensive set of vibration tests of a highway bridge which was renovated and upgraded for strengthening. Changes made to the bridge included the removal of overstressed rubber bearings causing a continuous, joint-less structure. The bridge essentially consisted of precast pre-tensioned inverted T-beams carrying a concrete deck slab on 
top. For instrumenting the bridge, a grid of points measuring only vertical acceleration was chosen, dividing the bridge into four strips in the transverse direction and six strips in the long direction. For the forced vibration test a shaker with a range of providing $0.5 \mathrm{kN}$ at 1.5 to $30 \mathrm{~Hz}$ was used and was located at the one-third span position on one walkway for convenience and maximum effect. Frequency response functions were obtained from the test by dividing the cross power spectrums with the auto power spectrums. Modal parameters were obtained using the SDOF circle fit and the Eigensystem Realization Algorithm procedures. In the pre-upgrade test 5 vibration modes were observed in the range of 0-20 Hz. In the post-upgrade tests lower mode frequencies appeared to be significantly higher. Evaluating these using the modal assurance criterion (MAC) it was observed that well-coordinated modes had values greater than 0.9 whereas the poorly coordinated ones had less than 0.5 . Results from these tests were also updated consequently in a finite element model. This updated FEM model was able to estimate the load carrying capacity more reliably than before. The authors were able to clearly observe the added rotational restraint in the modes following the structural changes.

\section{Modal Analysis and Modelling of Highway Bridges (Halling et al., 2004)}

In this research, two highway bridges in Utah were tested using forced and ambient vibrations. Bridge 1 was long-span flyover type structure and was tested by using a drop weight, and ambient vibrations. Bridge 2 was a two span bridge with a skew of 7 degrees and end to end elevation of about 2 meters. Instrumentation deployed on the first bridge included 10 removable accelerometers and 21 channels of strong motion force balanced accelerometers permanently attached to the structure. Of these 21 channels, 18 channels were located on the deck and bents of the bridge with 3 channels and the recorders located at a free-field site about 140 meters away from the bridge. Forced vibration testing was conducted using an eccentric-mass shaking that was mounted on the east extension of bent 7. The forcing function, ranging from 0.5 to $20 \mathrm{~Hz}$ was applied at 45 degrees to the longitudinal axis of the bridge.

Natural frequency estimates obtained from forced, ambient and a computer generated finite element were compared for the first 10 modes and were found to be highly comparable. For the second bridge, thirty-four channels of data were recorded during both ambient and forced vibration testing. Seven of those channels were longitudinal, seven vertical, and twenty transverse. The bridge was excited from ambient vibration where a $1800 \mathrm{~kg}$ drop weight was positioned in three different locations. 
Similarly, for this bridge a computer generated FEM model was compared with the ambient and forced tests showing a variation of no more than $4 \%$. The authors further suggested a long term study for the temperature and traffic effects on the monitoring system.

\section{Overview of a Modal-Based Condition Assessment Procedure (Wang et al., 2005)}

This article dealt with the process of characterizing the physical condition of a structure. A representative bridge in Ohio was selected as a case study to illustrate the procedure discussed in this paper. This condition assessment was performed by conducting modal and truck load testing followed by a finite element modelling and calibration. The bridge in question consisted of three spans and five girders with cover plates spaced at certain distances. For the modal test, 15-20 accelerometers with a range of $0.025-800 \mathrm{~Hz}$, were placed on the bridge deck above a given girder line and the bridge was systematically excited using an instrumented hammer while acceleration data was recorded. Data was acquired from these sensors using a Hewlett-Packard (HP) VXI data acquisition system. Locations of all output sensors were established based on the results of the nominal finite element modal. In the instrumentation scheme, two configurations were used where sensors of girder 2 and 5 were switched due to traffic restrictions:

A Bridge-Girder Condition Indicator (BGCI) was also calculated. This is simply the displacement profile of the instrumented girders and is computed by multiplying the flexibility matrix by a load vector. The BGCIs were used in the convergence study to determine how many modes are required to completely characterize the behavior of the bridge and were used along with the frequencies, mode shapes, and strain data in calibrating the finite element model. All the data captured was processed in MATLAB using routines in the frequency domain. Experimental and analytical mode shapes were correlated using the MAC. The finite element model was calibrated using an objective function. The authors observed that the standard calibration procedure developed for this bridge can be used and modified for another bridge in the absence of experimental data and would still yield more accurate results.

Real-Time Wireless Vibration Monitoring for Operational Modal Analysis of an Integral Abutment Highway Bridge (Whelan et al., 2009)

In this study a large-scale wireless sensor network was deployed for ambient vibration testing of a single span integral abutment bridge to derive in-service modal parameters. The bridge 
consisted of a thick reinforced concrete slab supported by four integral abutment girders with a single span. A total of 20 data points were monitored with dual-axis accelerometers:

The general test setup consisted of twenty dual-axis accelerometers deployed alongside eleven reusable strain transducers that were interfaced with twenty wireless sensor nodes. Acceleration responses of the bridge from ambient excitation were measured using accelerometers mounted directly to the web of each girder. A duration of three minutes and six seconds (186 seconds) was specified with an effective sampling rate of $128 \mathrm{~Hz}$. Excitation of the structure was provided only by ambient environmental loads and vehicular traffic. In this study, output-only system identification from the accelerometer time histories was performed using the Modal Analysis on Civil Engineering Constructions (MACEC) software package. This software analysis package permits the use of both the classical Fourier-based Frequency Domain Decomposition (FDD) with peak picking as well as the stochastic subspace identification (SSI) method for deriving mode shapes, natural frequencies, and damping ratios. A solid model of the bridge tested consisting of the steel superstructure, concrete slab, abutments, and railings was also drafted in AutoCAD 2004 from the as-built Department of Highways drawings. Finite element analysis of the model was performed using the FEMPRO software package by ALGOR Incorporated. The overall objective of this study was to evaluate the performance of the wireless sensor network. Real-time streaming of 40 channels synchronized with each other was achieved successfully.

\section{Recent Perspectives in Dynamic Testing and Monitoring of Bridges (Cunha et al., 2012)}

This research was an extensive review of some of the recent aspects in dynamic testing and monitoring of bridges. Three of the case studies within this article are summarized here.

\section{Millau Viaduct:}

This large cable-stayed road-bridge in France was used to perform ambient vibration testing using four seismographs including triaxial accelerometers. These seismographs, duly synchronized using GPS sensors, were placed outside the deck. With the purpose of identifying as many modes of vibration as possible, essentially of vertical and transversal bending nature, two of those triaxial sensors were used as references (sections R1 and R2) and kept in fixed positions, whereas the other two were successively placed in each one of the remaining 26 sections schematically indicated in. The seismographs were programmed using a laptop to acquire signals with a sampling rate of $100 \mathrm{~Hz}$ in periods of $960 \mathrm{~s}$ every $20 \mathrm{~min}$, the last $4 \mathrm{~min}$ being used. 
A singular value decomposition of the data showed the existence of a large number of modes in the frequency range of 0.1-1.0 Hz. Different stochastic modal identification methods such as the p-LSCF (poly-reference Least squares Complex Frequency-domain estimator) and SSICOV (Covariance-driven stochastic subspace identification) were used to obtain modal parameters.

\section{Pedro e Inês Footbridge:}

This slender structure with metallic arch spans and a rectangular box cross section was an interesting prospect in studying the dynamic structural behavior. According to preliminary studies developed at design stage it was stated that this bridge would be prone to excessive lateral and vertical human-induced vibrations, and so the structure was built with necessary precautions in order to enable the subsequent installation of several dampers. The monitoring system included six piezoelectric accelerometers, a signal conditioner, an uninterruptable power supply and a digital computer, stored inside one of the bridge abutments and transmitting measured records of vibration every $20 \mathrm{~min}$ through the Internet to a central computer. The signals were then organized in a database that can be accessed at any time by post-processing tools in LabVIEW (National Instruments Corporation Austin, Texas). To satisfy the main objective of this monitoring system, a website was developed, which allowed the visualization of the time signals of the six accelerometers and subsequently monitoring the vibration levels of the structure.

During the construction phase several dynamic tests were conducted including ambient, free vibration and crowd tests. Results from these tests were the basis for fine tuning the finite element model and subsequent final design of dampers. It was observed that a jump in the peak acceleration is observed at a certain number of pedestrians that is consistent with codified literature (HIVOSS). Damping estimates were also calculated for the damper design.

\section{Infant D Henrique Bridge:}

This bridge was composed of two mutually interacting fundamental elements: a very rigid prestressed reinforced concrete box beam, supported by a very flexible reinforced concrete arch. The dynamic monitoring system of the Infante D. Henrique Bridge was composed of 12 force balance accelerometers installed inside the deck box girder. Three sensors equip each of four sections, one to measure the lateral acceleration and two for the vertical acceleration at the downstream and upstream sides. 
Acceleration time series with a length of 30 minutes sampled at a rate of $50 \mathrm{~Hz}$ were recorded. Temperature sensors were also installed to eliminate any temperature effects in the modal parameter estimation. Three different identification algorithms, FDD (Frequency Domain Decomposition), SSI-COV and p-LSCF were used.

\section{A Sensor Instrumentation Method for Dynamic Monitoring of Railway Bridges (Bačinskas et} $\underline{\text { al., 2013) }}$

In this article, researchers conducted experimental and numerical analysis using forced and ambient vibration testing techniques. The bridge located in Lithuania, carried two railway tracks built on prestressed concrete sleepers and crushed stone ballast. The structural system of the bridge consisted of a composite steel-concrete deck rigidly fixed to the reinforced concrete abutments. For the instrumentation, a Brüel\&Kjær LAN-XI system was used for measuring the vibration parameters of the real structure. In the test, accelerometers were fixed to the control pads attached to the surface of the bridge superstructure. Field tests of the bridge were conducted using excitations induced by dropping a weight and by ambient traffic excitation loadings. During the tests vertical and horizontal lateral accelerations and displacements at mid-span of the girders were measured.

The purpose of the impact vibration tests was to extract bridge resonant modes and corresponding frequencies to be used for comparison with those obtained from the ambient vibration test and by numerical simulation. The collected test data was analyzed using the Brüel\&Kjær software program to determine main modal characteristics of the bridge superstructure: accelerations, mode shapes, frequencies of forced and free vibrations, damping ratios. The bridge response to dynamic excitation was measured as acceleration-time records. The measured acceleration response was filtered with a $30 \mathrm{~Hz}$ low-pass filter. A numerical modal analysis of the tested bridge was also performed using commercial finite element software Staad.Pro. It was observed that the numerical model predicted five modes below $20 \mathrm{~Hz}$ whereas experimental data was only able to pick up four. Experimental and numerical data were also found to be comparable to each other. The authors further suggested updating their numerical model with the experimental data to predict future condition. 
Finite Element Modal Analysis and Vibration-Waveforms in Health Inspection of Old Bridges (Rahmatalla et al., 2014)

Researchers in this article introduced a nondestructive vibration based approach known as the Operational Response and Waveform Analysis (ORWA) in order to validate predictability of the finite element model. The bridge investigated in this study was a composite steel I-girder and concrete deck bridge with a single span over a small natural creek in Iowa. The structure was not rated structurally deficient during its previous bridge inspection, but was deemed functionally obsolete and received a low sufficiency rating, meaning it was intolerable and required highpriority corrective action. For the ORWA test, five roving uniaxial accelerometers and one fixedreference accelerometer were used to record an extensive grid of 440 response points including 70 per beam, 10 per diaphragm, and 20 per abutment.

Accelerometers were fixed to the bottom flange of the beams and diaphragm members using magnets, and brackets were fixed to the face of the abutment walls with epoxy so that the vertical acceleration of the walls could be measured. A field impact test using a $5000 \mathrm{lbf}$ impulse hammer was also conducted on the bridge as a baseline approach with the goal of getting more confidence with the ORWA test. For the impact test, three $5000 \mathrm{~g}$ range triaxial accelerometers and six $100 \mathrm{~g}$ range uniaxial accelerometers were used to determine the dynamic response of various structural elements. Typically, accelerometers were fixed to structures with a magnet, but in some cases, where rust and dirt or concrete existed, wax was used with a magnet. An IOtech Zonic Book/618E Data Analyzer and an IOtech/650U were used to acquire data and construct the frequency response function (FRF). MEscope software was used to calculate the peak frequencies and associated mode shapes and to animate data. A numerical model of a bridge similar to a healthy form of the field-tested structure was then created using ANSYS. The ORWA testing was validated by both the impact test and the ANSYS model showing comparable bending modes.

\subsection{Serviceability Assessment}

In the section above a summary of the research articles with different vibration testing procedures was outlined. The topic of vibration testing is now further expanded to incorporate vibration aspects specifically on footbridges. The main reason in conducting an extensive serviceability assessment analysis on pedestrian bridges, has to do more with human comfort than with the structural integrity of the bridge. Human comfort on footbridges has been studied by 
several researchers in light of existing codes and guidelines (Bacinskas et al., 2014; Caifeng \& Linxiong, 2015; da Silva et al., 2007; Dey et al., 2015; El-robaa et al., 2015; Gudmundsson, 2008; Honda, 2007; Sadeghi \& Kueh, 2015; Salgado et al., 2014; Grandić, 2015; Van Nimmen et al., 2010; Živanović et al., 2006). The major pre-cursor to a sudden interest in the field of vibration serviceability was the London Millennium Bridge as discussed earlier. Since then, numerous researchers have tried to investigate the dynamic response to a footbridge under different types of force loading either analytically or experimentally or both. The following section discusses a few of these investigations:

\section{Modal Testing and FE Model Tuning of a Lively Footbridge Structure (Živanović et al., 2006)}

This was one of the first published articles where physical vibration testing and finite element modeling of footbridges was reported. The selected structure located in the country of Montenegro, was a steel box girder multispan footbridge. It was previously observed that at particular design load combinations stresses are induced in the structure close to allowable vibration limits in the cross sections. The bridge, despite being stiffened earlier, did not show a huge shift in these vibration frequencies. For a more detailed investigation the researchers developed a finite element model and recorded the modes shapes. Following the FE model, a FRFbased modal test was conducted on the bridge using only limited instrumentation, but multiple measurement points and a shaker for the excitation source. After this, an ambient vibration test was also conducted to obtain modal parameters. For the FRF modal test, the Non-Linear Least Squares (NLLSI-M) method was employed and for the ambient vibration test The Canonical Variate Analysis (CVA) was used. Analyzing the results from the tests, it was revealed that the experimental results were consistent with each other but there was a discrepancy with the FE model resulting in low natural frequency estimates. This was fixed by model tuning some of the stiffness parameters from the model, resulting in good correlation.

\section{Vibration Analysis of Footbridges Due to Vertical Human Loads (da Silva et al., 2007)}

The main idea behind this research article was to develop a design methodology to better evaluate the footbridge's user comfort and safety. A finite element model of a footbridge in the city of Rio de Janiero, Brazil was created on ANSYS. Dynamic forces on the model were simulated using Fourier Series equation for time varying forces incorporating a harmonic force dynamic amplifier $\alpha_{\mathrm{i}}$. The authors further suggested that human activities such as dancing, jumping and 
aerobics only excite the first mode whereas concentrated forces such as people walking may excite higher vibration modes. Two criteria for human comfort are suggested: Acceleration limits based on the ISO standards and a time dependent harmonic force component that coincide with the fundamental frequency. These in turn result in the formulation of a resonance response function, which is in turn incorporated into the model.

A total of four load cases are evaluated: 1) one resonant harmonic load applied at maximum amplitude point depicting a walking load, 2) a static and harmonic combination of time dependent loads depicting another walking load, 3) a moving time dynamic load simulated by six different forces applied at various intervals depicting a more realistic walking load and 4) an extreme load depicting a crowd. The natural frequency from a single degree of freedom model was verified by the finite element model. It was observed that acceleration limits from various international standards were not exceeded when only one person walked the footbridge, however, the acceleration limits did exceed the limits in the case of a large number of pedestrians such that it causes enormous problems related to human comfort.

\section{Serviceability Assessment of Three Lively Footbridges in Reykjavik (Gudmundsson et al., 2008)}

In this research article, researchers tested two post-tensioned concrete footbridges and one steel footbridge for vibration levels of which only one of the post-tensioned structures were discussed in this particular article. These bridges were located in Reykjavik, Iceland. The authors evaluated and updated the finite element models of the bridges with previously performed experimental vibration testing. Various load cases were implemented to assess serviceability of these bridges. These included pedestrians walking and running at specific frequencies. Several configurations were tested and then compared with the design standards. For the first test bridge, peak accelerations and response factors were compared with the Eurocode prestandard and ISO 10137 and results were found to satisfy the criteria. The load cases were also generated in the finite element model and peak accelerations graphed against experimental values, Bachmann values and the Eurocode standard. It was observed that the finite element model was the lowest in the single pedestrian case and was very accurate in the single runner case.

In the second bridge only running load cases were evaluated due to a higher frequency first mode. It was observed that the Eurocode limits were exceeded by a single runner at and around the resonant frequency compared to a packed crowd. The authors suggested the use of a 
probabilistic approach instead of using deterministic load models and binary pass-fail acceleration criterion.

\section{Vibration Serviceability of Bongossi Wood Pedestrian Bridge (Honda, 2012)}

In this short research article, the author conducted an analytical serviceability test on a timber footbridge located in city of Kitakyushu in Japan. Field tests were also conducted on the bridge through ambient vibrations, impact loading and resonant walking and running vibrations. A pedestrian model was then developed in FORTRAN using equations of motions through the direct integral method. Results obtained were compared with the Ontario Code and BS5400. It was observed that both standards satisfied the measured and computed results.

\section{Vibration Serviceability of Footbridges: Evaluation of the Current Codes of Practice (Van} Nimmen et al., 2010)

A comprehensive study is published in this article whereby the authors evaluate two different design codes, the European guide HIVOSS and the French guide SÉTRA using data obtained from a finite element model as well as from vibration testing. The two codes are discussed in context of their relationship to each other given a four step methodology: 1) human induced vibrations as defined by the equivalent number of pedestrians, 2) footbridge dynamic characterization whereby both codes explain different criteria for added mass and damping ratios, 3) vibration calculation or maximum acceleration under maximized loading, reduction factor and resonance frequency and 4) vibration level evaluation under various comfort levels. A total of 8 footbridges were studied. Results from the ambient tests and numerical model are tabulated for all the bridges. Two of the eight total footbridges were provided in detail. It was concluded that small variations in the finite element model result in different code evaluation. Therefore, a modified load model was suggested given uncertainties in the design stage. It was observed that damping values also influence the predictions but are difficult to estimate in the design stage. An in situ identification of modal parameters was finally suggested.

Field Load Testing and Structural Evaluation of Steel Truss Footbridge (Bacinskas et al., 2014)

In this article, researchers tested a newly constructed steel truss footbridge in Lithuania. The bridge was simplified to a simple span model due to the location of elastomeric bearings at the ends. A finite element was created in Staad.Pro to assess static and dynamic characteristics. For the static test consideration, the bridge was loaded with precast concrete slabs and during the 
dynamic test the span was excited by a drop weight and by pedestrians. An array of 26 sensors was used to instrument the bridge. In the static tests, the mid span deflection and the residual displacements were observed to be in accordance with the finite element model. In the dynamic test, measured natural frequencies were lower than code specified limits and therefore required a vibration comfort evaluation. Codes used in this research were adopted from the Lithuanian standards, which are in effect based on the Eurocode. Numerical simulations in finite element model were compared with the code standards and a three people running experiment. Results were found to be below the specified limits and in good correlation with the experimental values.

Serviceability Assessment of the Góis Footbridge using Vibration Monitoring (Salgado et al., $\underline{\text { 2014) }}$

In this paper the authors looked into the different design codes that provide standards for serviceability assessment of a footbridge. The footbridge used in this study was a timber arch structure located in Portugal. The bridge was instrumented with accelerometers at 31 measuring points including both transverse and vertical directions. The mode shapes obtained for the footbridge displayed excessive flexibility in the lateral direction. The authors noted good estimation of natural frequencies and damping ratios given small standard deviations. To determine the acceleration levels three different pedestrian cases were evaluated. This included a case with pedestrians walking at $1.4-1.8 \mathrm{~Hz}$ then one with $2.7-3.1 \mathrm{~Hz}$ and the last case with pedestrians jumping on the bridge at frequencies of 2.0-2.5 Hz. Peak acceleration values and the natural frequencies were then compared to several different codes and standards. The limits specified in these standards were satisfied in some cases and required further acceleration assessment in other cases. The authors also observed that using RMS accelerations a better parameter of pedestrian comfort level can be evaluated. It was finally concluded that current codes and standards are not fully applicable and a further study was proposed.

\section{Ambient Vibration Survey and Vibration Serviceability Evaluation of Footbridges (Caifeng et} $\underline{a l ., 2015)}$

In their research Caifeng \& Linxiong tested 37 footbridges in various districts of China by conducting an ambient vibration test followed by a vibration serviceability evaluation based on two design codes. Modal analysis was conducted on the acquired data by peak picking the natural frequencies based on the Frequency Domain Decomposition Technique. Several different forms 
of footbridges were tested such as single or multi span with spans ranging from 20 meters to 120 meters. It was observed that most of the footbridges have fundamental frequencies in the range of 2-7 Hz where the lowest frequencies correspond to single span pre-arched footbridges and the highest frequencies correspond to short span bridges with piers.

Damping ratios were also calculated using the half power bandwidth method. For the vibration serviceability the authors used the Chinese Industrial Standard CJJ69-95 with the British Standard 5400 to evaluate the serviceability of the footbridge. In the evaluated criteria, acceleration limits needed to be satisfied $\left(a_{\max } \leq 0.5 \sqrt{f}\right)$ if the fundamental frequency was between 3-5 Hz. If the fundamental frequency was more than $5 \mathrm{~Hz}$ the criterion was automatically satisfied and if the fundamental frequency was less than $3 \mathrm{~Hz}$, then it was not satisfied at all. In their evaluation, the authors suggested reinforcement for increased stiffness or vibration absorbers for footbridges that did not pass the serviceability criteria.

\section{Evaluation of Human-Induced Vibration of Continuous Footbridge (El-Robaa et al., 2015)}

In this research human-induced vibrations are evaluated through the "footfall" phenomena using finite element modelling. The footfall analysis method as published by "The Concrete Centre" CCIP-016 is a rigorous analysis method for calculating structural response from a single walking person. A response factor $(\mathrm{R})$ is calculated which is simply a multiplier of the base line curve for RMS acceleration as defined in the British Standard 6472-1992. This corresponds to the level of average threshold of human perception in continuous vibration. For the purpose of their research, the authors modelled a total of 15 footbridges composed of hinged supported composite steel beams with 2 equal spans and a reinforced concrete composite slab deck. The various cross sections were optimized for deflection and strength and were evaluated by the footfall analysis. In the analysis a range of walking frequencies from 1-2.8 Hz were generated through a forcing function and induced into specific nodes in the structure. Based on the acceleration components of these nodes the total response factor is obtained. This model was simulated for all the range of frequencies and the maximum response factor was obtained. It was found that for all cases, heavily trafficked indoor bridges did not satisfy the CCIP-016 limit cases. Different finished weights of the bridges also corresponded to varying response factors. The authors noted a key point that in some cases a frequency of $3 \mathrm{~Hz}$ and above in a small span bridge with low finished weight was 
not satisfied by the footfall analysis despite being satisfied by other international standards such as the AASHTO LRFD standards.

Vibration Serviceability Analysis of Aluminum Pedestrian Bridges Subjected to Crowd Loading (Dey et al., 2015)

In this article, the notion of serviceability assessment was extended to incorporate aluminum pedestrian bridges that were constructed in house. The two bridges of spans 12.2 meters and 22.9 meters were assembled with bolted connections from aluminum members and had equal width and height dimensions. A total of twelve accelerometers were instrumented on the structures both laterally and vertically. Modal tests on the structure were conducted and the natural frequencies and damping ratios were recorded. For serviceability considerations several pedestrian tests were conducted with varying configurations. The tests were also repeated several times for added redundancy and statistical significance. The test results were compared with 4 different standards namely: Eurocode 5, British National Annex, SÉTRA and HIVOSS. Each of the codes were used to obtain loading scenarios and acceleration limits.

Natural frequencies from the structure were verified with the critical limits to assess the requirement of a dynamic analysis. It was observed that except for the shorter bridge in vertical frequency range, every other case had to be evaluated for a secondary dynamic check. An important observation made by the authors was the likely increase of vibration due to the successive harmonics of the walking frequency resonating with the structures natural frequency. A further study in damping uncertainty was suggested by the authors.

\section{Serviceability Assessment of Composite Footbridge Under Human Walking and Running Loads} (Sadeghi \& Kueh, 2015)

The aim of this research study was to generate fundamental research knowledge on the vibration characteristics of slender composite footbridge structures under human running and walking loads for further evaluation with current design standards. In this study the authors employed modal analysis using finite element model of the composite bridge. Human walking and running was simulated using the harmonic forces as expressed in the Fourier time domain. The serviceability criterion was adopted from ISO standards, where 2 specific items: peak acceleration limits and the harmonic force component were checked. For the harmonic case a resonance response function was defined in terms of the reduction factor. The finite element model was 
created in SAP2000. The ground motion induced by the pedestrian was applied through the application of a three component (longitudinal, lateral and vertical) force at the middle of the bridge. It was observed that the first fundamental frequency coincided with only the third harmonic of the walking load and second harmonic of the running load. From the results it was seen that the maximum accelerations were below the peak limits specified in ISO, Ontario Code and the BS 5400. The deflection limits were also checked using LRFD provision of $L / 1000$. All of the various checks were satisfied for the model.

\section{Serviceability Verification of Pedestrian Bridges Under Pedestrian Loading (Grandic, 2015)}

This research article identifies the lack of defined dynamic models for pedestrian loads in serviceability as the main disadvantage in current European codes. As a result, the authors propose a design load model that can fulfil this void. Pedestrian walking forces in all the three directions (vertical, lateral and horizontal) were represented by periodic components in the Fourier series. Each force is defined by a step frequency variable, phase shifts and Fourier coefficients/dynamic load factors. These dynamic load factors were defined separately in various other articles and were tabulated in the paper. However, these factors were not taken into account in different international codes and forces are estimated based only on a step frequency that coincides with the structure's natural frequency. The authors defined three different load models: 1) single pedestrian, 2) group of pedestrians and 3) continuous pedestrian stream. A sample footbridge model in Zagreb, Croatia was modelled in DARK software developed at University of Rijeka and the proposed load models were implemented. Maximum accelerations and natural frequencies were obtained from closed form solutions as well as from the software. Results were found to be lower than codified limits and difference between closed form solutions and software was not more than $3.8 \%$ in any case.

\subsection{Summary}

The literature in this section highlights the work performed in the area of dynamic testing and serviceability assessment of pedestrian bridges. Studies discussed above show how dynamic properties of a bridge structure are obtained from experimental testing methods using different data processing techniques. Forced vibration tests and ambient vibration tests are commonly used to obtain the dynamic data. Dynamic data obtained from a pedestrian bridge is then used to assess its serviceability condition. It was shown above how the key parameters in a serviceability 
evaluation are the peak accelerations and the natural frequency limits of the structure. Guidelines and codes exist that limit these parameters of a given structure.

However, most of the existing research has focused on European standards and very few comprehensive case studies are present that suggest vibration serviceability in American standards. It is also seen that minimal guidance is provided by AASHTO on vibration serviceability and as such none of the present literature discuss serviceability assessment under the light of AASHTO design guidelines (AASHTO, 2009). Another observation from the research review is that effects of ambient conditions such as temperature are also not investigated. Temperature effects on structures have been studied earlier but not in the light of vibration serviceability.

The work detailed in this thesis will incorporate different European and American codes and perform an in-depth serviceability assessment of a lively footbridge. Comparisons of the European and American standards will be made in an effort to further research in the topic of Vibration Serviceability Assessment. Effects of temperature on the assessment would also be briefly explored. 


\section{DYNAMIC CHARACTERIZATION OF RUFFNER FOOTBRIDGE}

This section will discuss the complete dynamic characterization of the selected pedestrian bridge. This is the methodology section where the procedure for obtaining dynamic properties of natural frequencies, mode shapes and damping for the structure as well as the various guidelines are presented. An outline of this section is provided in the subsection below.

\subsection{Investigation Procedure}

There are several steps that outline the overall investigation procedure. The following model briefly explains these steps:

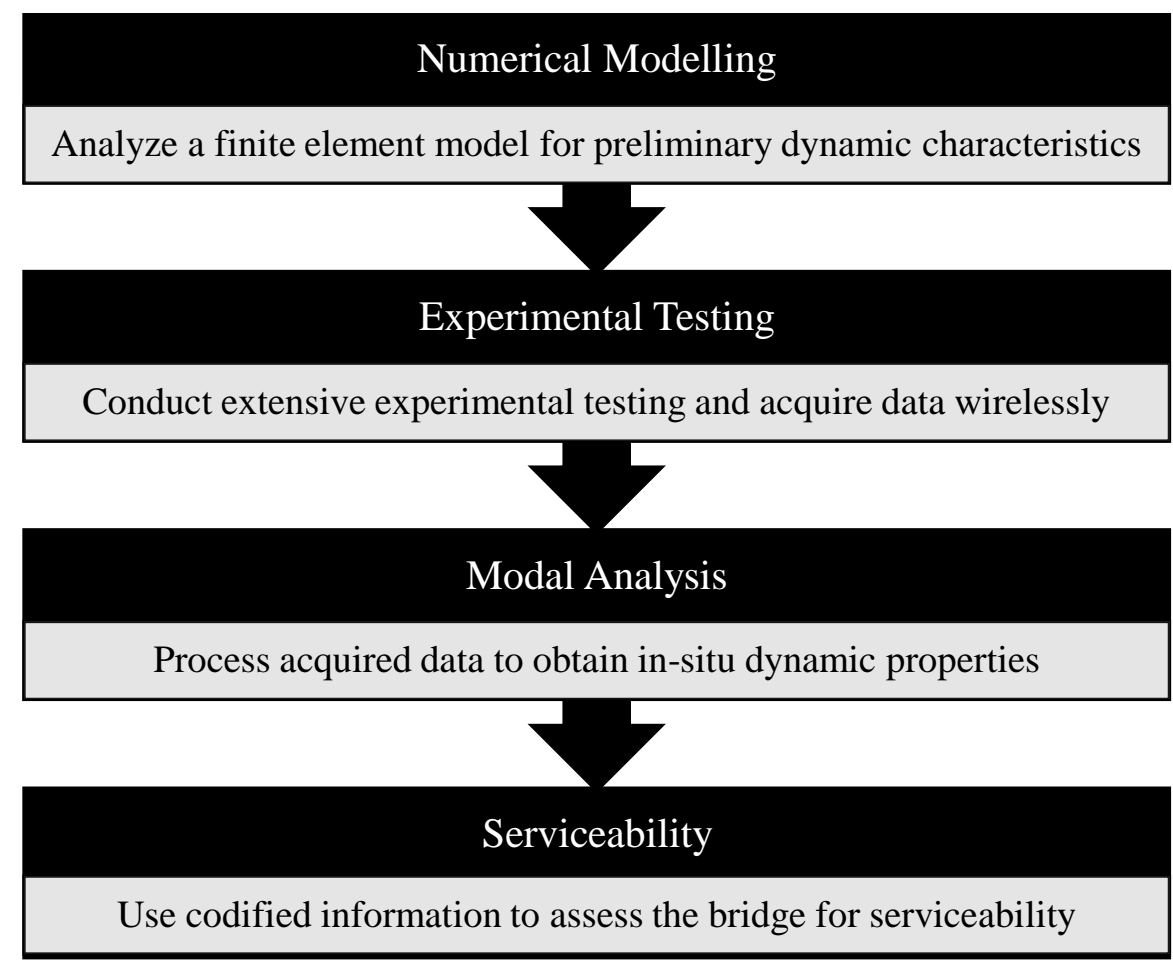

From the investigation matrix above, the document will initially present results from a preliminary finite element model followed by the experimental procedure, the subsequent data analysis and finally the serviceability assessment of the footbridge. The numerical model developed in SAP2000 acts as a base model whereby the initial dynamic properties and mode shapes are estimated. These are then subsequently used to guide the experimental and serviceability testing plan. Data acquired from this plan is then processed in MATLAB using modal analysis techniques to obtain the in-situ dynamic characteristics. These characteristics are then finally incorporated into the selected codes and guidelines for serviceability assessment. 


\subsection{Description of the Selected Footbridge}

The footbridge investigated in this study is one of two footbridges located south on Emmet Street on US 29 in the city of Charlottesville, Virginia. It is located on the grounds of the University of Virginia and crosses US 29, linking Ruffner Hall with South Newcomb Road on the other side. Figure 7 shows pictures of the footbridge from different angles.

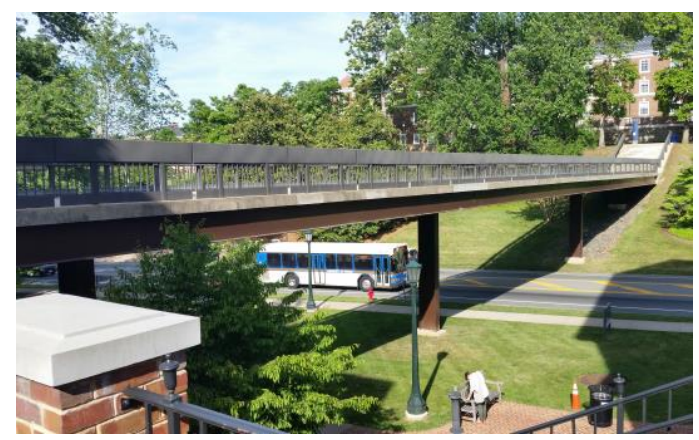

(a)

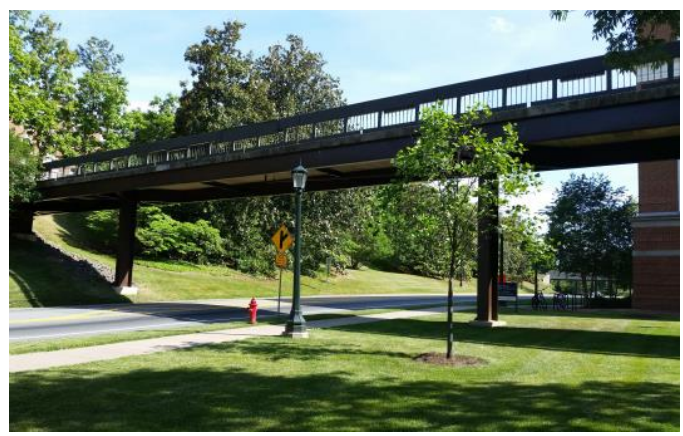

(b)

Figure 7. Ruffner Bridge a) View from Bavarro Hall (South West Side); b) View from North West sidewalk below

Due to its location at the center of University of Virginia, it is frequently used by students, staff and faculty members and is highly trafficked during major sporting events (e.g. football games) and ceremonies (e.g. commencement). Constructed in 1973, the bridge is essentially a multi-span steel girder with a cast in place concrete deck. Twin girders support the concrete deck with diaphragms at the pier intersection and cross-bracing at regular intervals along the length of the structure. Other important features of the bridge include its cantilever on both ends and parapets with railings. These features play an important role in the overall mode calculation as the cantilever causes added flexibility of modes and the parapets will cause greater stiffness for any torsional motion. Some of these features can be seen in Figure 8 below.

The total length of the bridge is $190 \mathrm{ft}$ with successive five spans of $15,52,70,38$, and 15 $\mathrm{ft}$ each. The total width of the bridge is $16 \mathrm{ft}$ including $1 \mathrm{ft}$ parapets on each side. The center span girders have a thin cover plate on the bottom flange for added strength and stiffness. The unit weight of concrete, reported from the drawings, is 110 pcf with a deck slab thickness of $10.5 \mathrm{in}$. Based on measured geometry and available plans, total mass of the superstructure per unit length is estimated at $1879.0 \mathrm{lb} / \mathrm{ft}$. A simple (scaled) layout of the bridge plan is shown in Figure 9. 


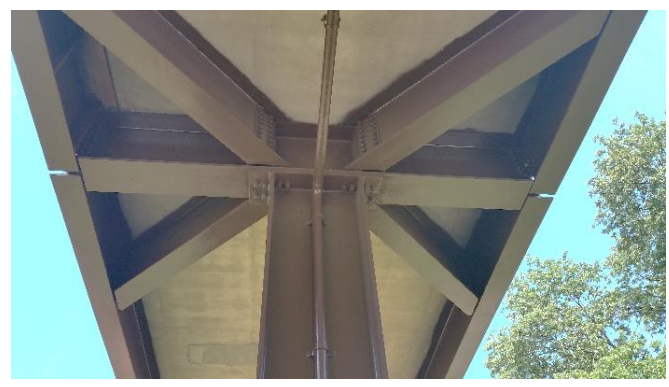

(a)

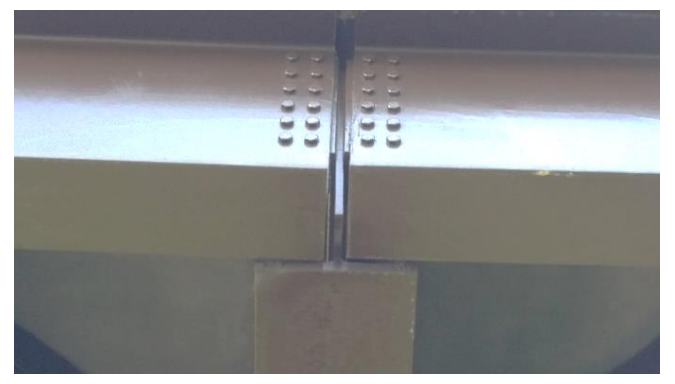

(b)

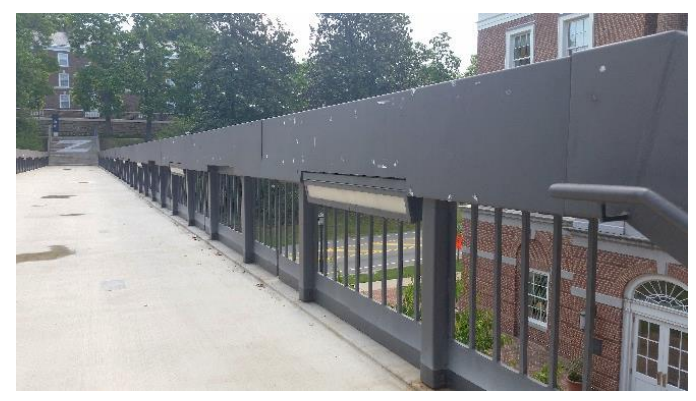

(c)

Figure 8. (a) Cross bracing, (b) Girder connection between each span, (c) View of the side parapet and railing

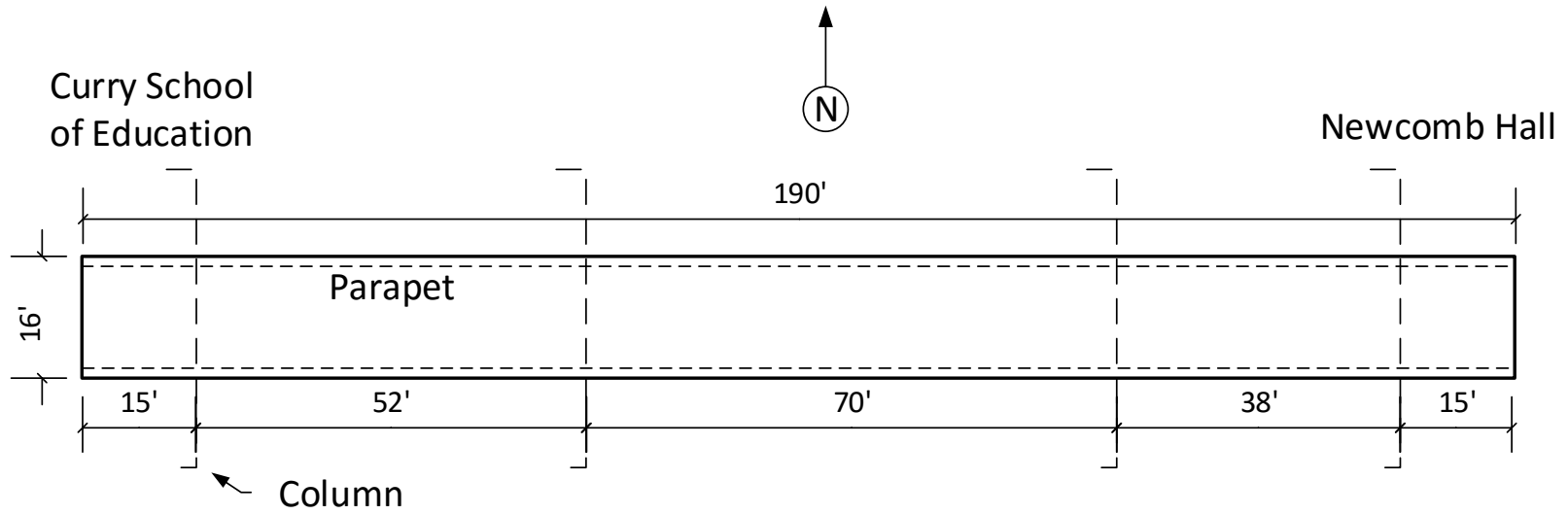

Figure 9. Bridge layout

\subsection{Preliminary Finite Element Analysis (FEA) (Gheitasi et al., 2016)}

A 3D finite element model of the pedestrian bridge was developed using (CSI, 2013). Due to the uncertainties in the material and structural properties, engineering based estimation was used for some parameters in the model development. The model was developed using four-node shell elements with a thin-plate formulation incorporating both plate and membrane behavior for the deck, and two-node frame elements with general beam-column formulation for the girders. Each 
node possessed six degrees of freedom and relevant rotational and/or translational degrees of freedom were restrained at certain joints to replicate the observed boundary conditions as closely as possible. Linear elastic material behavior was employed for both steel and concrete materials and composite action between the deck and the girders was enforced through node sharing. A mesh sensitivity analysis was also carried out to ensure the adequacy of the mesh refinement. A free vibration analysis was performed to determine natural frequencies and mode shapes of the footbridge. The six lowest vertical and transverse modes of vibration were computed and are illustrated in Table 9. The frequencies and mode shapes derived from the FEA models were used in planning the instrumentation of the bridge for ambient vibration tests described in the following sections (Gheitasi et al., 2016).

Table 9. Frequencies and modes of vibrations derived from the preliminary finite element model

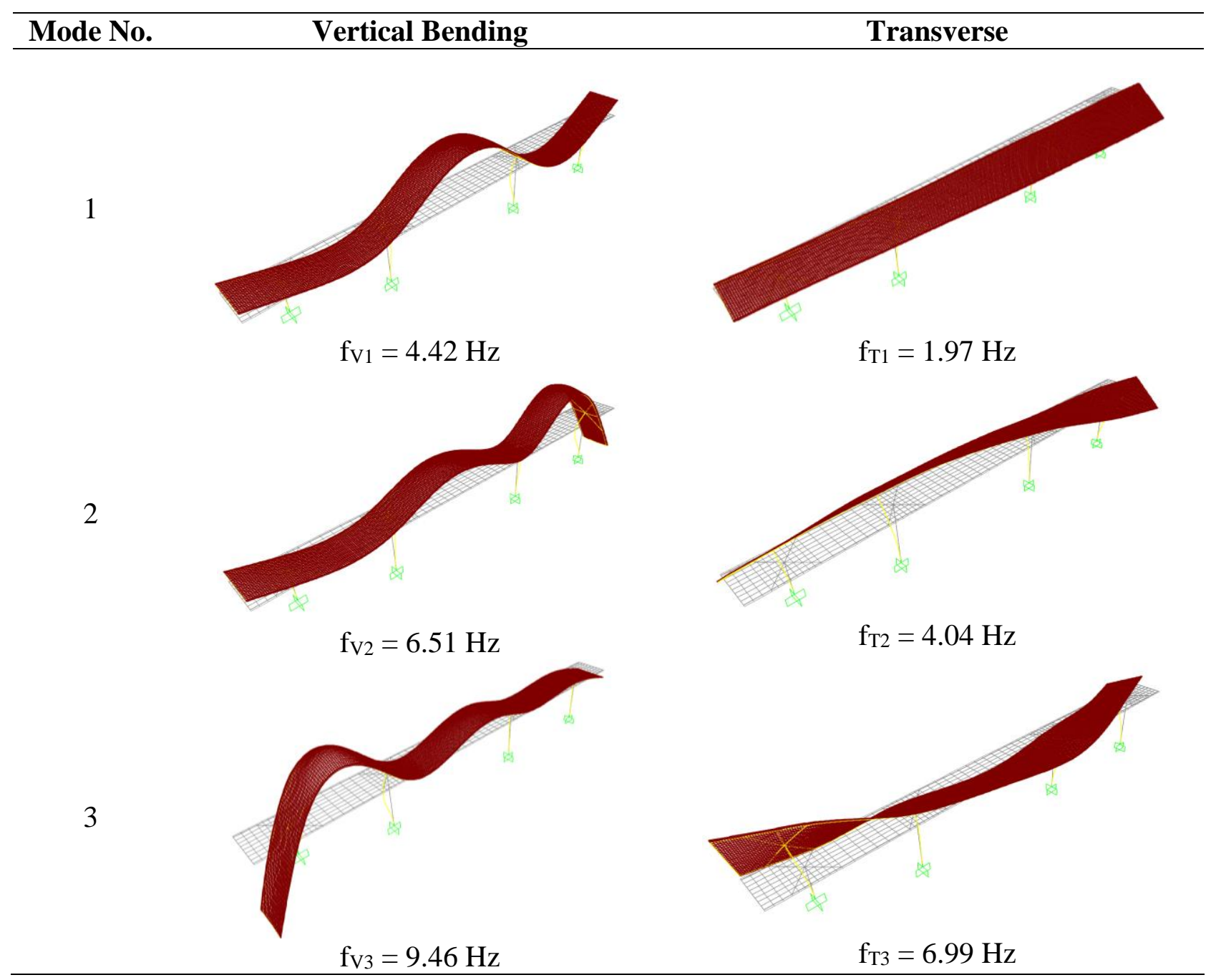




\subsection{Experimental Test Setup}

Results from the preliminary finite element model were used to plan the instrumentation scheme for the experimental tests. Transducer and impact locations were chosen based on the relative modal displacements of the meshed grid in the finite element model. Points near zero modal displacement or "nodes" were not instrumented such as the deck part that was on top of the columns. Testing was conducted at three different times of the year. These included testing the structure in July, October 2015 and then January 2016. On each of these test days, testing usually started around 11:00 am and continued until approximately 4:00 pm. Since the tests were conducted in three different times of the year instantaneous temperature of the structure was also measured. Temperature and its effects on the vibration data will be discussed in Chapter 6 .

\subsubsection{Equipment}

Instrumentation used for the vibration test primarily consisted of a set of accelerometers connected to a wireless data acquisition system. For the forced vibration test an impact hammer was used which was also connected to the same data acquisition. The DAQ system used was manufactured by Bridge Diagnostics Inc. (BDI) and included the BDI Win-STS software that was installed on a standard laptop manufactured by Panasonic (Model CF350). Accelerometers used were made of micro-machined capacitive sense element and were also BDI manufactured. They had a range of $\pm 2 \mathrm{~g}$ and a differential sensitivity of $1 \mathrm{~V} / \mathrm{g}$. These sensors were connected by 20 feet long field-grade instrumentation cables to a BDI STS-Wi-Fi node where a total 6 nodes were used with an individual capacity to hold 4 transducers providing a total capacity of 24 transducers capable of simultaneous data collection. However, due to the limited number of accelerometers that is 10 , only a few of these nodes were used at a time. These were then connected wirelessly to a single base station which in turn was connected to the laptop using a Wi-Fi signal.

For the forced vibration test, an impact hammer was used that was manufactured by PCB Piezotronics and had a force range of $0-5000 \mathrm{lbf}$. The hammer was of model 086D50 and included two different impact tips (a stiffer and softer tip). The hammer was connected to a PCB Piezotronics signal conditioner model 480E09 using BNC cables which in turn was connected to the BDI node using another BNC - Alligator clip cable. The interface for this connection was made possible using a universal sensor input plug supplied by BDI. 
Calibration files for each of the sensors connected to the DAQ system were factory computed and installed in the software. Calibration for the impact hammer was based on the impact hammer voltage to force sensitivity and was done manually. Data collected from the DAQ system was saved automatically by the BDI-STS software as an excel file. This file was then further imported and the data processed for modal analysis in MATLAB ("MATLAB," 2015). Temperature measurements on the deck were taken using a digital laser infrared thermometer temperature gun and ambient temperature were recorded from weather.gov database.

\subsubsection{Instrumentation Plan}

As mentioned earlier, all vibration testing spanned over a period of 3 days and was conducted non-consecutively. Three different instrumentation plans were used for the test, where one was specific to impact and ambient data and the other two for serviceability:

Impact and Ambient Test: The first instrumentation plan was for the ambient and forced vibration test and hence had a much more refined mesh. A total of 18 vertical data points were used as accelerometer locations including 2 reference points and 2 impact hammer locations. Due to a limited number of sensors the grid was divided into half and the test repeated twice, one for the first half of the bridge and one for the other. This instrumentation scheme is shown below.

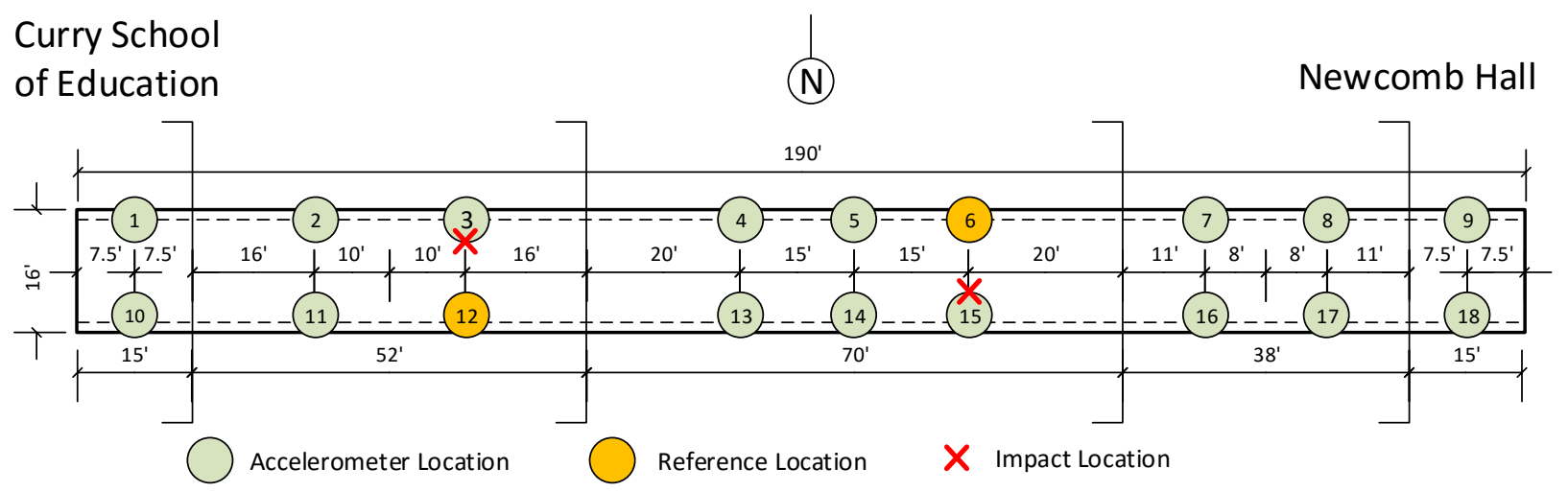

Figure 10. Ambient and impact tests instrumentation scheme

Table 10 shows the details of this test: 
Table 10. Impact and ambient test matrix

\begin{tabular}{cccccc}
\hline Test No. & Type & Attached Sensors & Impact Point & Duration (s) & Repetitions \\
\hline 1 & Impact & $6,12,1,2,3,4,10,11,13,14$ & 3 & 15 & 5 \\
2 & Impact & $6,12,1,2,3,4,10,11,13,14$ & 15 & 15 & 5 \\
3 & Ambient & $6,12,1,2,3,4,10,11,13,14$ & N/A & 900 & 1 \\
4 & Impact & $6,12,5,7,8,9,15,16,17,18$ & 15 & 15 & 5 \\
5 & Impact & $6,12,5,7,8,9,15,16,17,18$ & 3 & 15 & 5 \\
6 & Ambient & $6,12,5,7,8,9,15,16,17,18$ & N/A & 900 & 1 \\
\hline
\end{tabular}

Serviceability Test: In this test, two different testing techniques or plans were used. In the first technique groups of 1 and 9 people walked on the bridge at different walking frequencies as measured by a metronome (Gismart [Mobile application software], 2016). Accelerometers were placed in both vertical and transverse directions. Figure 11 shows the scheme:

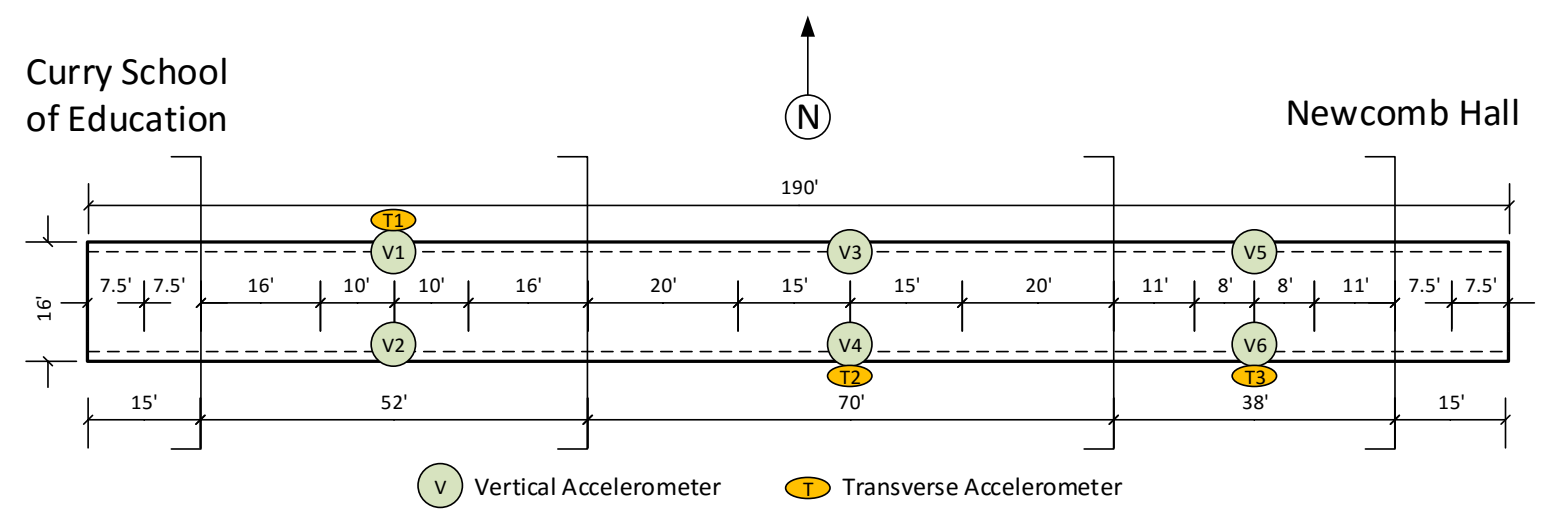

Figure 11. Serviceability first part tests instrumentation scheme

Table 11 shows the details of this test:

Table 11. Walking serviceability test matrix

\begin{tabular}{ccccc}
\hline Test No. & Type & No. of People & Walk Frequency $(\mathbf{H z})$ & Repetitions \\
\hline 1 & Serviceability & 9 & 1 & 3 \\
2 & Serviceability & 9 & 1.5 & 3 \\
3 & Serviceability & 9 & 2 & 3 \\
4 & Serviceability & 1 & 1 & 3 \\
5 & Serviceability & 1 & 1.5 & 3 \\
6 & Serviceability & 1 & 2 & 3 \\
7 & Serviceability & Crowd & N/A & 3 \\
\hline
\end{tabular}


For the second technique one pedestrian was used to create a jumping motion at a frequency of $2 \mathrm{~Hz}$ at each of the mid points of the span. The frequency of $2 \mathrm{~Hz}$ was chosen based on the fact that results from the previous serviceability tests showed peak accelerations at walking frequency of $2 \mathrm{~Hz}$. This was due to its second harmonic being close the bridge's fundamental frequency. Accelerometers were instrumented in a similar fashion as the first serviceability technique with few changes including removal of the longitudinal sensor and one transverse sensor to accommodate the added two vertical sensors. Figure 12 shows the instrumentation scheme:

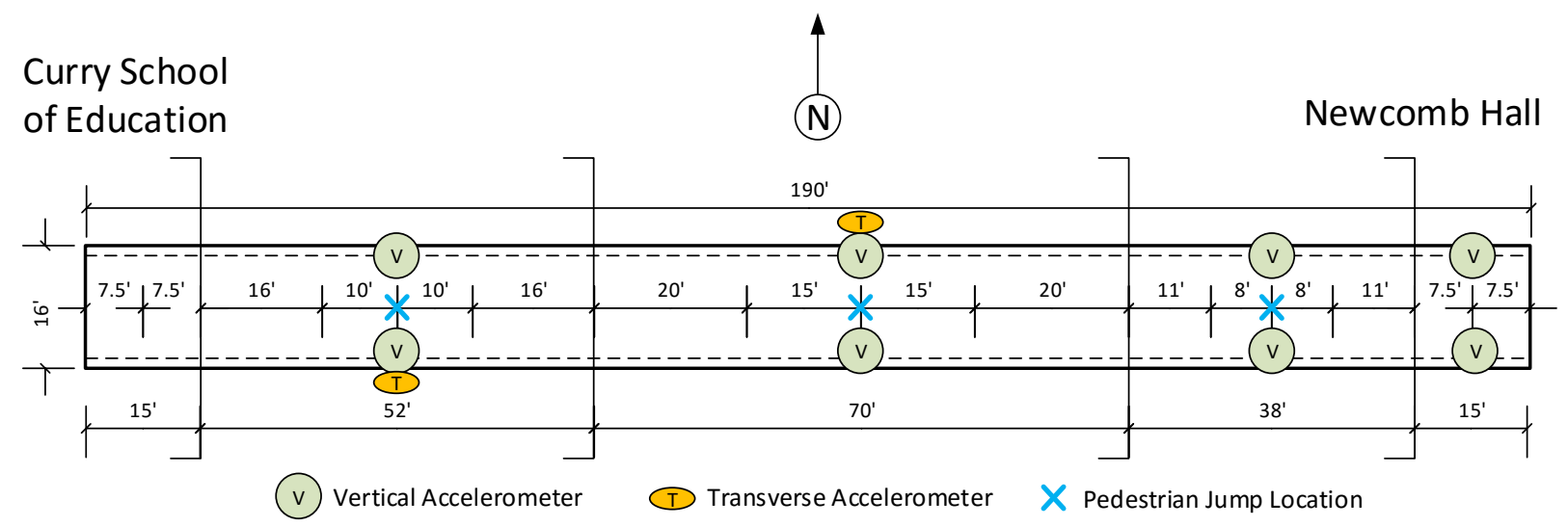

Figure 12. Serviceability second part tests instrumentation scheme

The testing matrix for this plan is shown in Table 12:

Table 12. Jumping serviceability test matrix

\begin{tabular}{ccccc}
\hline Test No. & Type & Location & Jump Frequency (Hz) & Repetitions \\
\hline 1 & Serviceability & Left Span (Curry) & 2 & 3 \\
4 & Serviceability & Center Span & 2 & 3 \\
9 & Serviceability & Right Span (Newcomb) & 2 & 3 \\
\hline
\end{tabular}

Sensor attachment and BDI node locations can be seen from Figure 13 below:

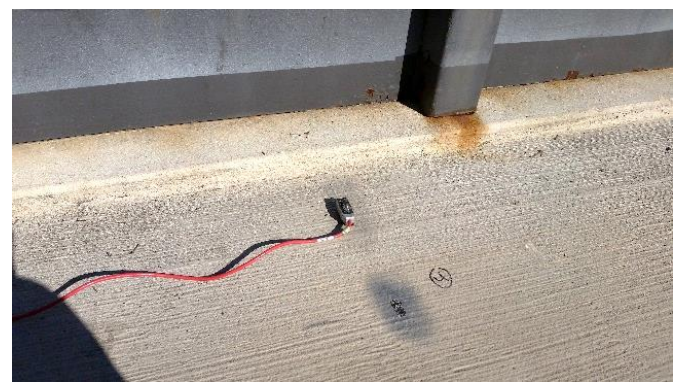

(a)

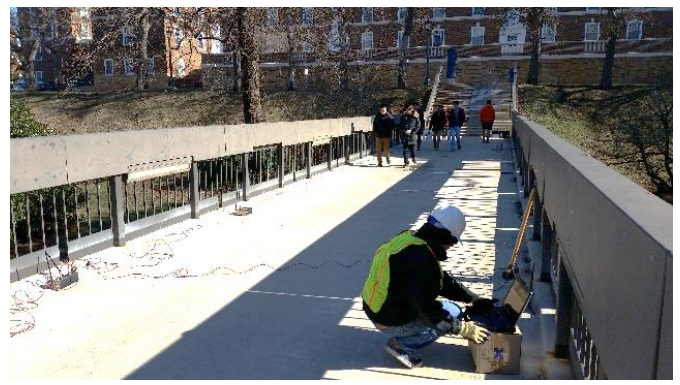

(b)

Figure 13. (a) Location 4 of accelerometer, (b) Node and base station shown on the left of the bridge 


\subsubsection{Testing Logistics}

In all the above tests, data collection time was changed according to each test. Therefore, for tests which had a quick impact followed by free vibration, a time of 15 seconds was chosen to completely capture the response. Serviceability tests where people walked from one end of the bridge to the other, collection time was changed from 30 seconds to 1 minute depending on the walking frequency. For example, at a walk frequency of $2 \mathrm{~Hz}$ when people moved faster and crossed the bridge in less time, data collection was shorter than for a walk frequency of $1 \mathrm{~Hz}$.

In all of the tests (impact, ambient and serviceability) a sampling frequency of $100 \mathrm{~Hz}$ was used. This was selected based on the range of frequencies obtained from the preliminary finite element model. Since the frequencies of interests lay between $0-10 \mathrm{~Hz}$, there was no need to have a high sampling rate. A sampling frequency of $100 \mathrm{~Hz}$ ensured that a range of $0-50 \mathrm{~Hz}$ of frequencies were obtainable from the response data based on the Nyquist theorem (Olshausen, 2000).

In the impact tests, a soft hammer tip was used. The hammer tips allow for the energy content of the force impulse to be tailored to deliver the desired range of frequency content (PCB.com, 2016). In this bridge only the first three modes were particularly of interest, as they corresponded to a low natural frequency range of 1-10 Hz. As there was no need to trigger higher modes, using a harder tip would have given excess data. As mentioned earlier, during all these tests, temperature was also measured throughout the day. This will be discussed further in the later sections.

The following Figure 14 shows images from the tests.

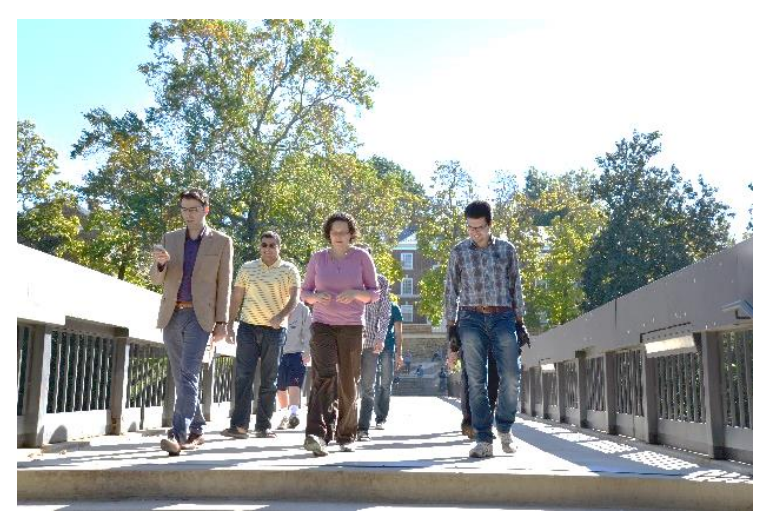

(a)

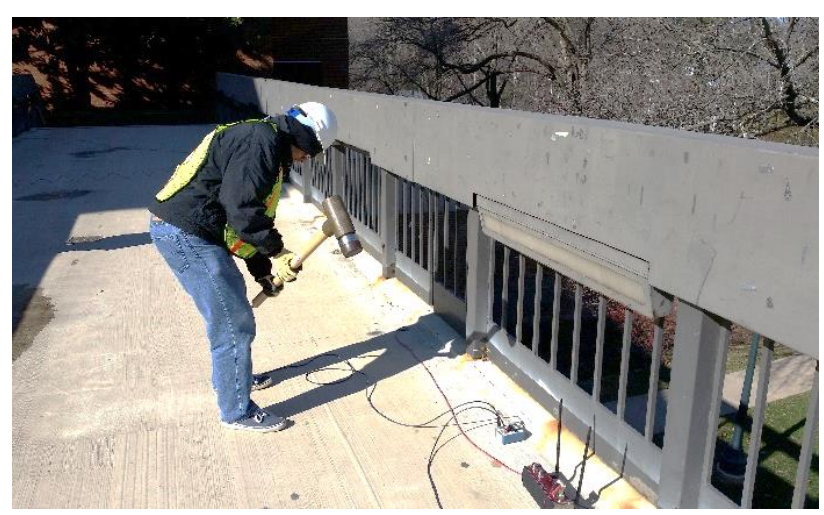

(b)

Figure 14. (a) Serviceability test with 9 people, (b) Impact hammer test 


\section{RESULTS AND DISCUSSION}

All of the test data was collected in BDI data acquisition software Win-STS ("Win-STS," 2011) and was then transferred and processed in MATLAB. Due to the wireless capabilities of the BDI hardware, a battery was used which caused power fluctuations in time domain data when set for an extended period of time. This issue was resolved in MATLAB by using the command "detrend," applied to all sensor data for consistency. The following Figure 15 shows an example:

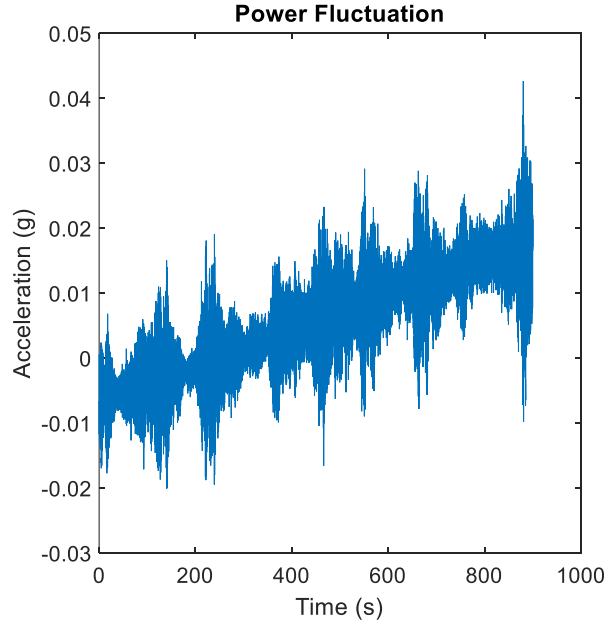

(a)

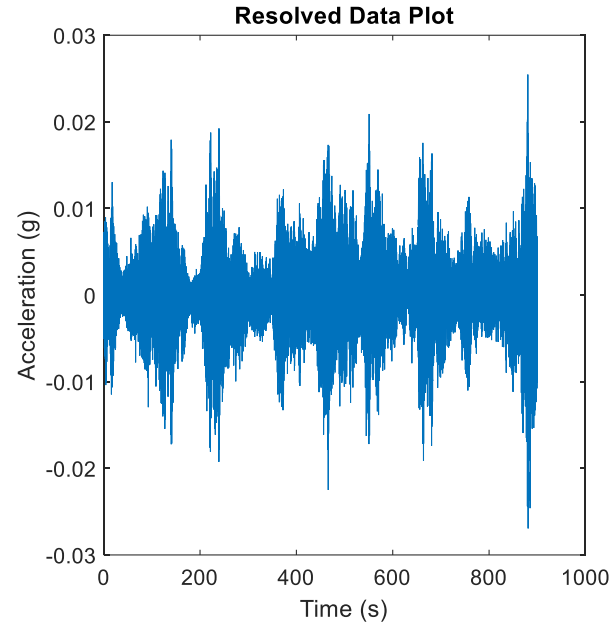

(b)

Figure 15. (a) Power fluctuation and (b) resolved ambient data for sensor 7

Filtering was performed to suppress any unwanted part of the signal. An $8^{\text {th }}$ order filter was applied using MATLAB to filter out undesired low and high frequencies. This was done by initially using a low pass infinite impulse response filter with a pass band of $30 \mathrm{~Hz}$ and then by using a high pass infinite impulse response filter with pass band of $0.5 \mathrm{~Hz}$. These can be seen below:
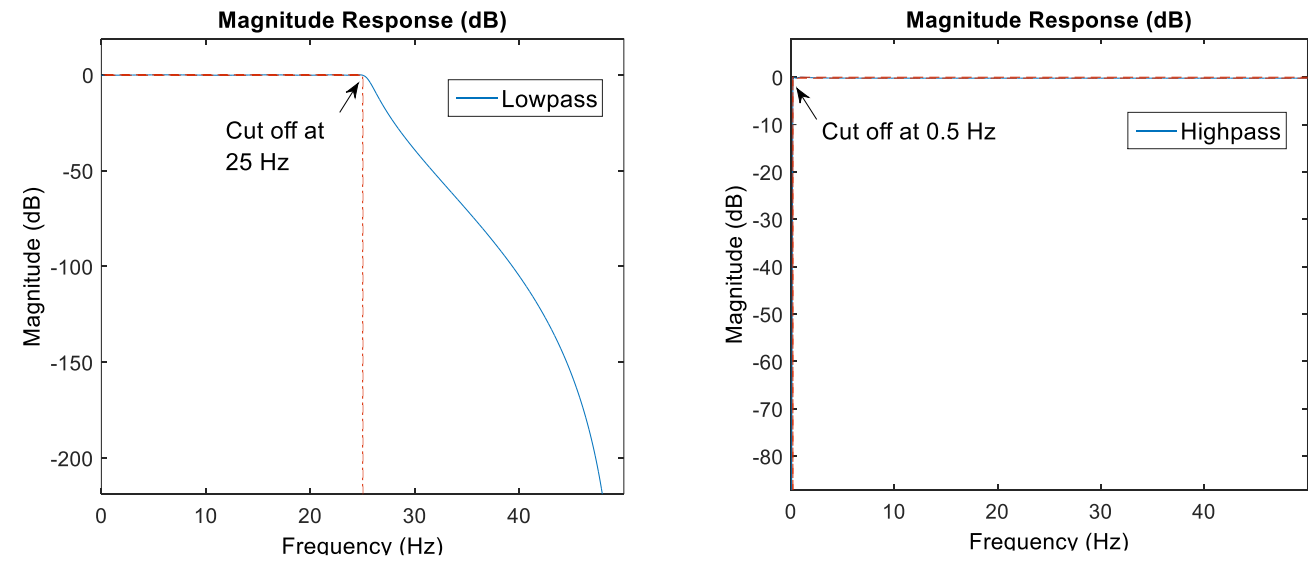

Figure 16. (a) Low pass filter at $30 \mathrm{~Hz}$, (b) High pass filter at $0.5 \mathrm{~Hz}$ 


\section{1. $\quad$ Forced Vibration Tests}

Forced vibration test data consisted of an input signal and output data. The input signal was the impulse force recorded upon impact from the impact hammer. The output data was the data from accelerometers, i.e. acceleration data in $g$ s, where $g$ denotes the gravity. Upon impacting the bridge, the impact hammer showed a peak resulting in the attached accelerometers showing peak accelerations followed by free vibration of the bridge. A sample of such a test is shown below:
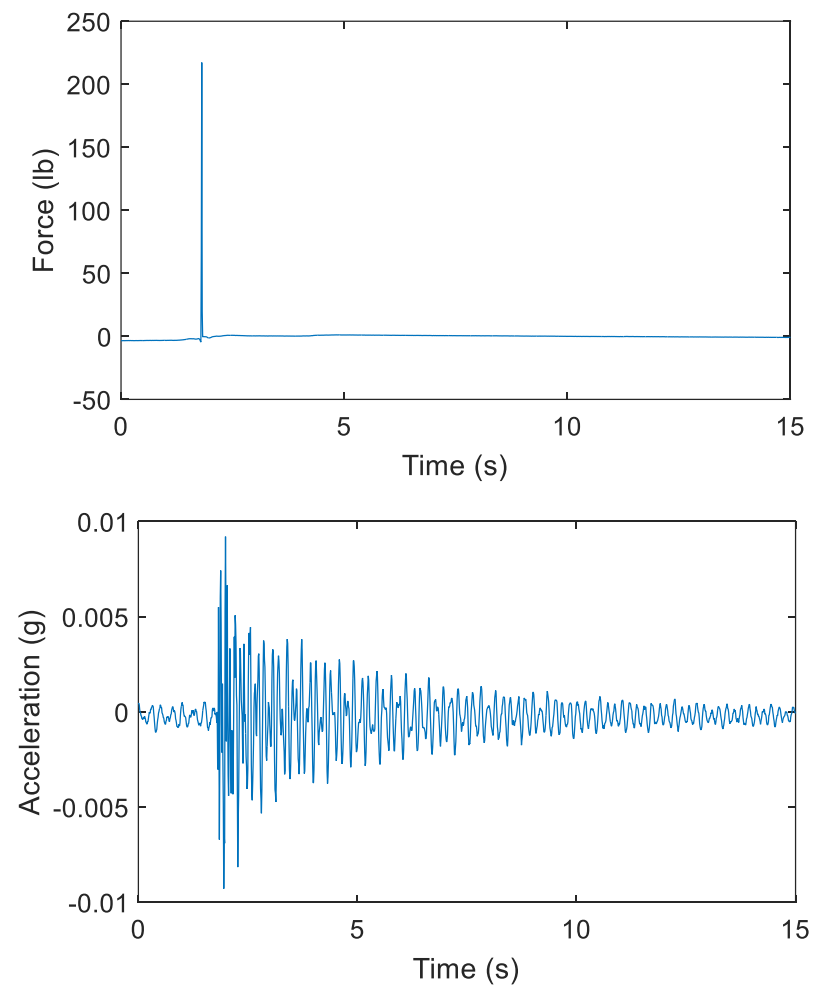

Figure 17. Sample impact hammer plot (top) and acceleration data from sensor 12

The above data was then processed in MATLAB. Using equation (6) and (22) we can write the frequency response in matrix form as follows:

$$
\left\{\begin{array}{c}
X_{1} \\
\vdots \\
X_{18}
\end{array}\right\}=\left[\begin{array}{ccc}
H_{1,1} & \cdots & H_{1,18} \\
\vdots & \vdots & \vdots \\
H_{18,1} & \cdots & H_{18,18}
\end{array}\right]\left\{\begin{array}{c}
F_{1} \\
\vdots \\
F_{18}
\end{array}\right\}
$$

Where $X$ is the response in terms of the frequency response function (FRF) as obtained from the accelerometers and $F$ being the impact force from the hammer. A total of 4 matrix equations are formed, each corresponding to the impact test from Table 10. In order to link the two sets of two impact test data, the FRF matrix is pre-multiplied by the reference FRF in each of the cases. These 
are then combined or added together to give a normalized mode FRF vector. Since the forcing locations in the test were 3 and 15, two columns vectors within the FRF matrix corresponding to $H_{3,1-18}$ and $H_{15,1-18}$ will be obtained. Each time domain data is also filtered and a window is applied. This was explained earlier in section 2.2.4. The overall procedure to obtain one FRF matrix can be seen from the schematic below (Note: The plots shown in the schematic only indicate the procedure performed and do not reflect the original data):

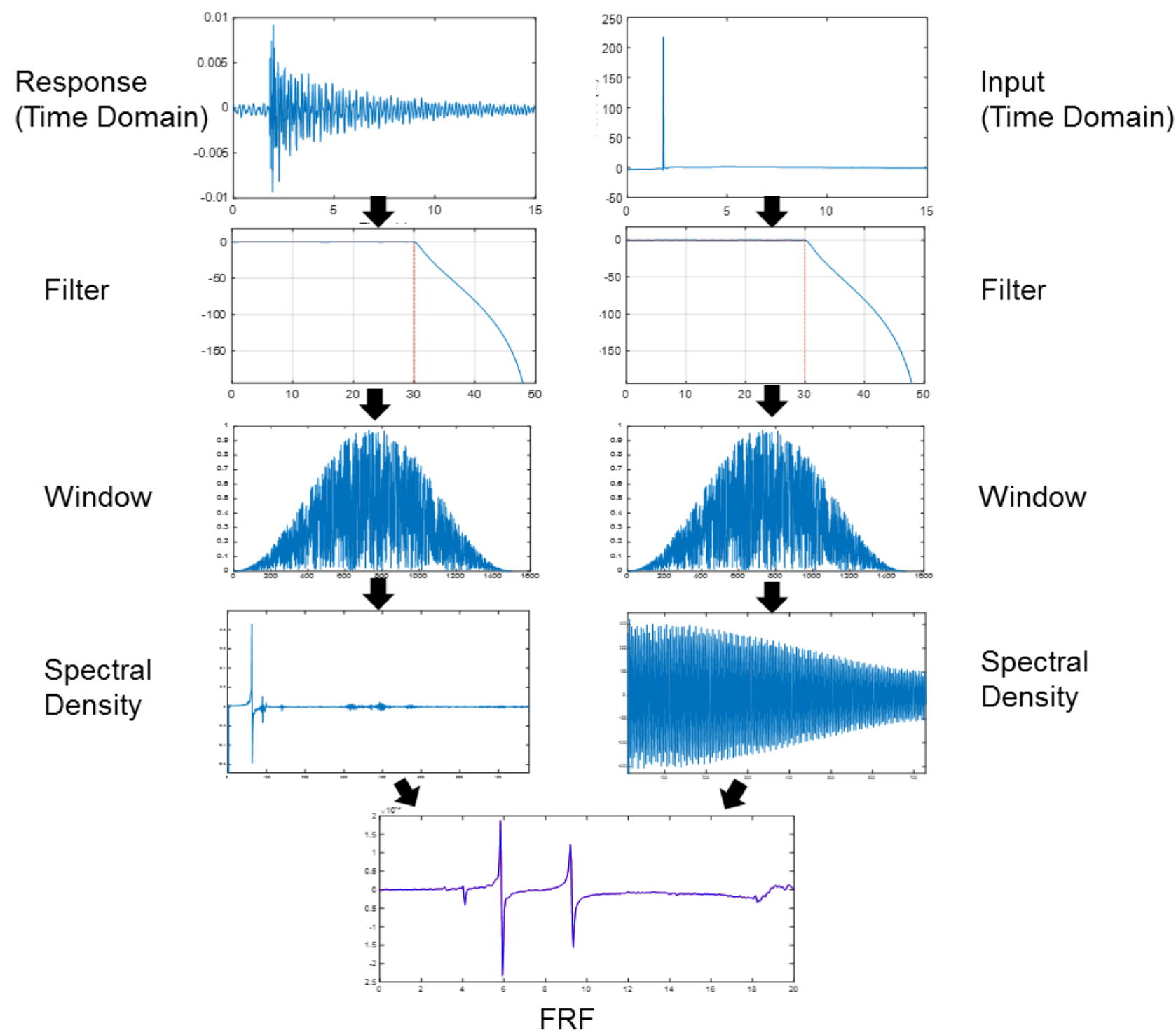

The two different FRFs using equation (22) are therefore obtained. It was decided to use only $\mathrm{H}_{1}$ data as resonance peaks (circled in red) were easily identifiable in $\mathrm{H}_{1}$ than in the $\mathrm{H}_{2}$ frequency spectrum. Figure 18 is an example of both of these FRFs and their resulting coherence: 

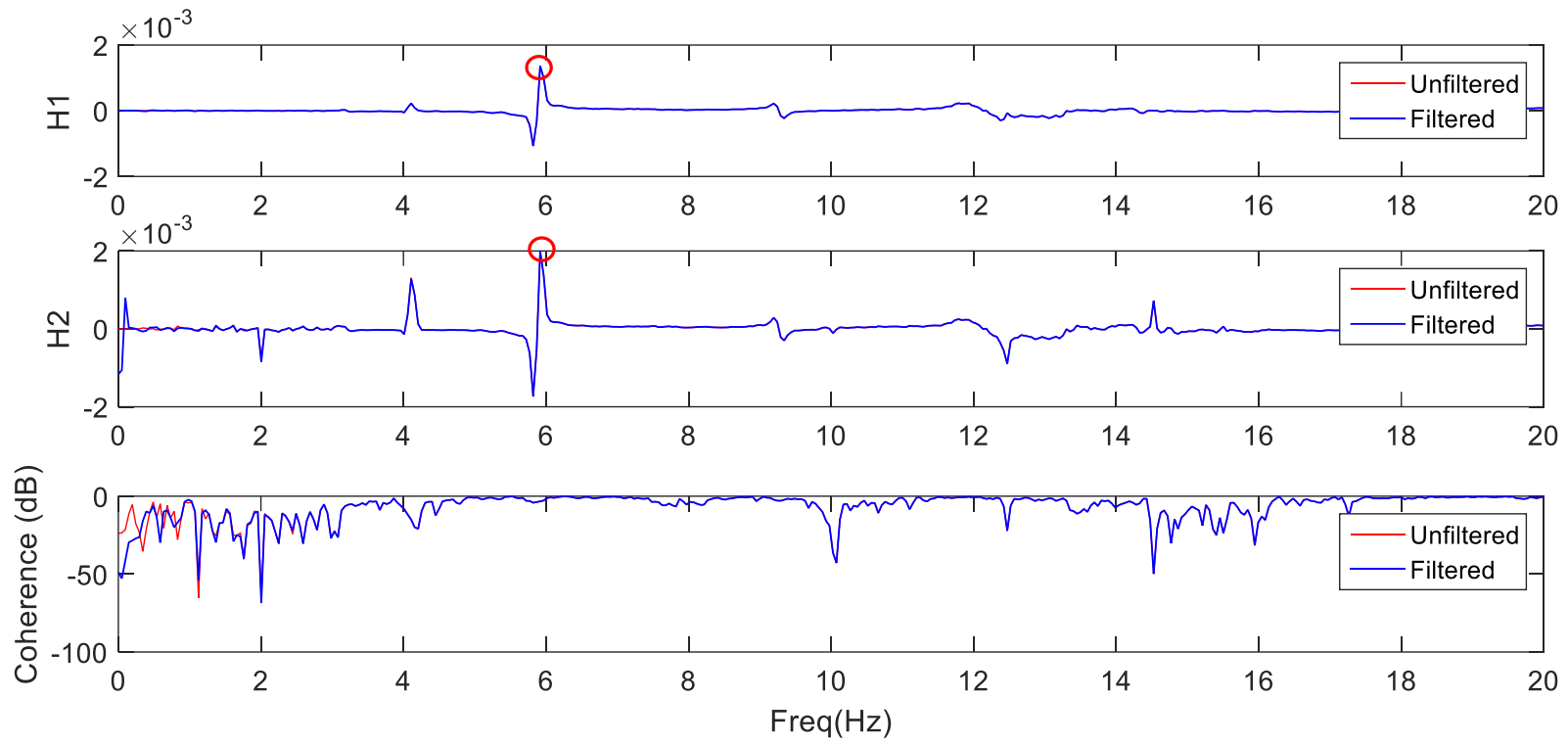

Figure 18. Frequency response function and coherence $\mathrm{H} 1 \& \mathrm{H} 2$ for impact location at 3 and sensor at 1

From Figure 18 above, the presence of peaks around 4 and $6 \mathrm{~Hz}$ can be observed with 6 (circled in red) being more dominant. This corresponds to the natural frequency of the bridge. However, it must be noted that a minor peak also exists around $9 \mathrm{~Hz}$ corresponding to the third natural frequency, but is not very clear. This will eventually appear in other graphs depending on the impact location and the response measured. It is also observed that coherence for the FRF is high showing that the impact force is very well correlated to the response. A summary of the first three natural frequencies and the damping ratios from the impact response plots for each test is shown in Table 14. Table 13 shows the averaged frequencies for each of the forcing locations.

Table 13. Summary of obtained natural frequencies

\begin{tabular}{cccc}
\hline Forcing Location & $\mathbf{1}^{\text {st }} \boldsymbol{f}_{\boldsymbol{n}}(\mathbf{H z})$ & $\mathbf{2}^{\text {nd }} \boldsymbol{f}_{\boldsymbol{n}}(\mathbf{H z})$ & $\mathbf{3}^{\text {rd }} \boldsymbol{f}_{\boldsymbol{n}}(\mathbf{H z})$ \\
\hline 3 & 4.102 & 5.869 & 9.341 \\
15 & 4.053 & 5.811 & 9.326 \\
\hline
\end{tabular}

Table 14. Complete list of picked natural frequencies and damping ratios

\begin{tabular}{cc|cc|cc|cc}
\hline $\begin{array}{c}\text { Forcing } \\
\text { Location }\end{array}$ & $\begin{array}{c}\text { Sensor } \\
\text { No. }\end{array}$ & $\begin{array}{c}\mathbf{1}^{\text {st }} \boldsymbol{f}_{\boldsymbol{n}} \\
(\mathbf{H z})\end{array}$ & $\begin{array}{c}\zeta \\
(\%)\end{array}$ & $\begin{array}{c}\mathbf{2}^{\text {nd }} \boldsymbol{f}_{\boldsymbol{n}} \\
(\mathbf{H z})\end{array}$ & $\begin{array}{c}\zeta \\
(\%)\end{array}$ & $\begin{array}{c}\mathbf{3}^{\text {rd }} \boldsymbol{f}_{\boldsymbol{n}} \\
(\mathbf{H z})\end{array}$ & $\begin{array}{c}\zeta \\
(\%)\end{array}$ \\
\hline 3 & 1 & -- & -- & 5.918 & 0.59 & -- & -- \\
3 & 2 & -- & -- & 5.82 & 0.50 & 9.194 & 0.48 \\
3 & 3 & -- & -- & 5.82 & 0.50 & 9.194 & 0.48 \\
\hline
\end{tabular}




\begin{tabular}{|c|c|c|c|c|c|c|c|}
\hline 3 & 4 & 4.108 & 0.44 & 5.82 & 0.51 & -- & -- \\
\hline 3 & 5 & 4.108 & 0.39 & 5.869 & 0.57 & -- & -- \\
\hline 3 & 6 & 4.108 & 0.43 & 5.869 & 0.53 & -- & -- \\
\hline 3 & 7 & -- & -- & -- & -- & 9.243 & 0.38 \\
\hline 3 & 8 & -- & -- & -- & -- & 9.194 & 0.41 \\
\hline 3 & 9 & -- & -- & -- & -- & 9.194 & 0.40 \\
\hline 3 & 10 & -- & -- & 5.918 & 0.60 & -- & -- \\
\hline 3 & 11 & -- & -- & 5.869 & 0.50 & 9.341 & 0.22 \\
\hline 3 & 12 & -- & -- & 5.869 & 0.53 & 9.341 & 0.26 \\
\hline 3 & 13 & 4.108 & 0.44 & -- & -- & -- & -- \\
\hline 3 & 14 & 4.108 & 0.48 & -- & -- & -- & -- \\
\hline 3 & 15 & 4.108 & 0.39 & 5.869 & 0.58 & -- & -- \\
\hline 3 & 16 & -- & -- & 5.967 & 0.21 & 9.341 & 1.12 \\
\hline 3 & 17 & -- & -- & 5.967 & 0.31 & 9.341 & 0.91 \\
\hline 3 & 18 & -- & -- & -- & -- & 9.341 & 0.47 \\
\hline 15 & 1 & 4.01 & 0.64 & 5.918 & 0.27 & -- & -- \\
\hline 15 & 2 & 4.108 & 0.59 & 5.82 & 0.41 & -- & -- \\
\hline 15 & 3 & 4.108 & 0.81 & 5.869 & 0.60 & 9.194 & 0.53 \\
\hline 15 & 4 & 4.01 & 0.93 & 6.553 & 0.23 & -- & -- \\
\hline 15 & 5 & 4.059 & 0.87 & 6.553 & 0.22 & -- & -- \\
\hline 15 & 6 & 4.059 & 0.89 & 6.553 & 0.23 & -- & -- \\
\hline 15 & 7 & 4.108 & 0.86 & 6.553 & 0.25 & -- & -- \\
\hline 15 & 8 & 4.108 & 0.79 & -- & -- & -- & -- \\
\hline 15 & 9 & -- & -- & -- & -- & -- & -- \\
\hline 15 & 10 & 4.01 & 0.64 & $5.92,6.553$ & 0.22 & -- & -- \\
\hline 15 & 11 & 4.108 & 0.59 & $5.82,6.456$ & 0.41 & 9.292 & 0.42 \\
\hline 15 & 12 & 4.108 & 0.81 & $5.82,6.456$ & 0.47 & 9.341 & 0.30 \\
\hline 15 & 13 & 4.01 & 0.83 & 6.456 & 0.32 & -- & -- \\
\hline 15 & 14 & 4.059 & 0.91 & 6.456 & 0.39 & -- & -- \\
\hline 15 & 15 & 4.059 & 0.87 & 6.456 & 0.36 & -- & -- \\
\hline 15 & 16 & 4.108 & 0.86 & 6.456 & 0.42 & -- & -- \\
\hline 15 & 17 & 4.108 & 0.84 & -- & -- & -- & -- \\
\hline 15 & 18 & -- & -- & -- & -- & -- & -- \\
\hline
\end{tabular}


It can be observed from Table 14 that some of the sensors displayed two closely spaced peaks or frequencies for the second mode such as sensor 10 upon impact at 15 (shown in the table as separation by a comma). This is a clear indication of the presence of two modes that have a natural frequency very close to each other. It is possible that one of these is a torsional mode similar to the $2^{\text {nd }}$ pure bending mode. Some of the sensors were located close to a node and were thus not able to pick up any of the natural frequencies such as sensor 18 upon impact at 15 . The damping ratios can be observed to range between $0.4-0.9 \%$. The values are reasonable but show large variation. The average damping for the structure is around $0.53 \%$.

Using the frequency response function's imaginary component as shown in equation (25), the mode shapes were also computed. An example of the imaginary parts of FRFs from two output points are provided below to show this procedure. In this case the goal is to identify the $2^{\text {nd }}$ mode shape. Peaks of imaginary parts of FRF at $2^{\text {nd }}$ natural frequency are identified in Figure 19 below.

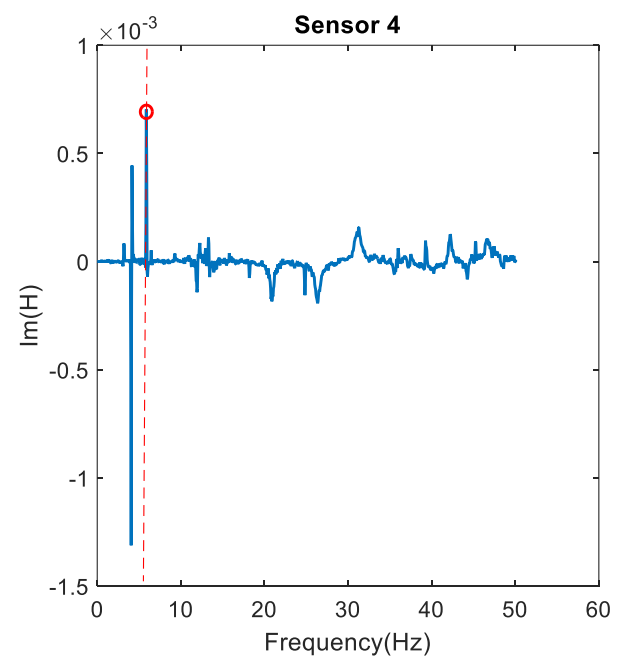

(a)

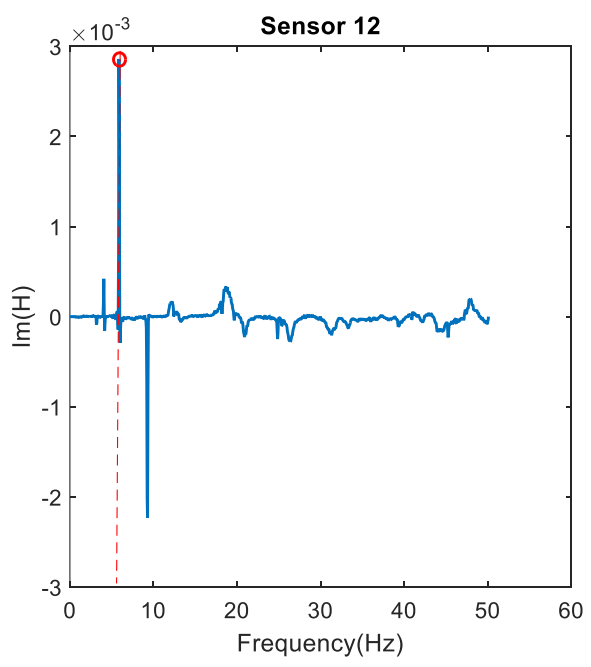

(b)

Figure 19. (a) Imaginary FRF plot for sensor 4, (b) Imaginary FRF plot for sensor 12

Using the above technique, the first 3 mode shapes are obtained from the data and are shown in Table 15. It is clearly observed that the first 2 mode shapes that are in pure bending are very similar but the third mode shape which is a torsional is not completely similar to the other. This could mainly be attributed to the low power output from the third mode in the frequency response function. It is possible that during the impact test, only the first two modes were excited and the third mode was not. From Table 14, the recorded third natural frequency occurs much less frequently than the first and second. This can also be seen graphically in Appendix IV FRF plots: 
Table 15. Obtained mode shapes for different impact points

Forcing Location

\subsection{Ambient Tests}

Similarly, the modal parameters were also obtained from the ambient test data. The ambient test data was collected in two different times of the year, one in the month of July and the other in the month of January so that the effect of temperature could also be studied. Following is the plot of singular values obtained from the ambient data in both cases:

Winter

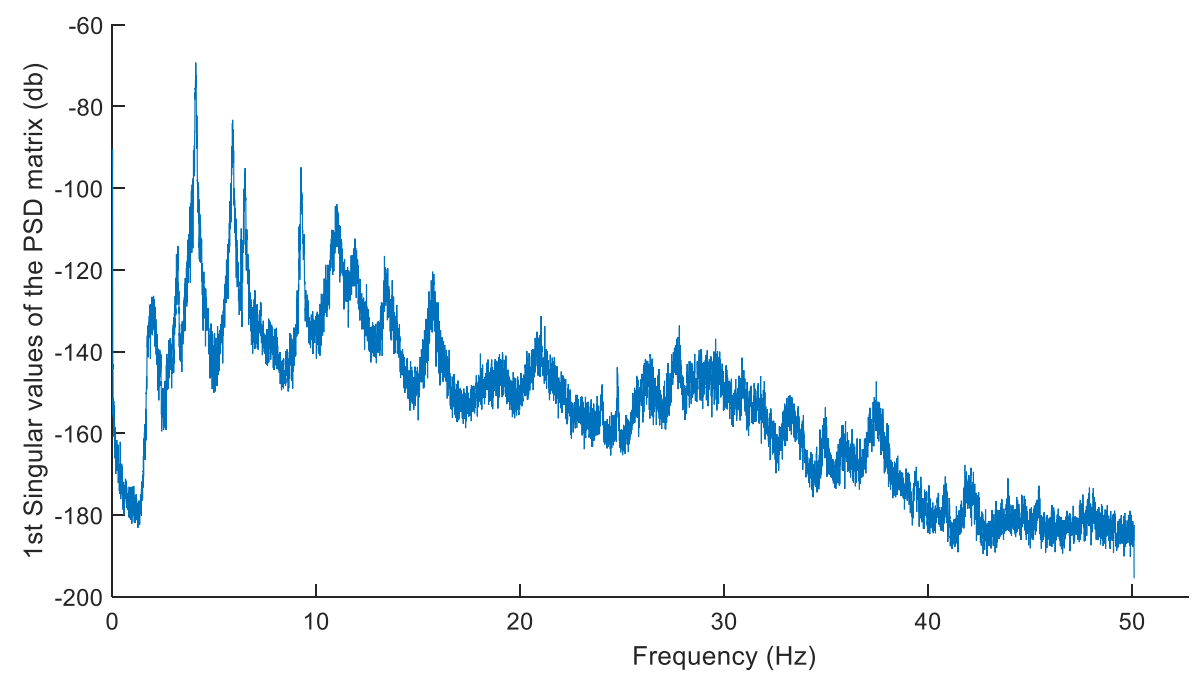

Figure 20. SVD plot of ambient test in winter 


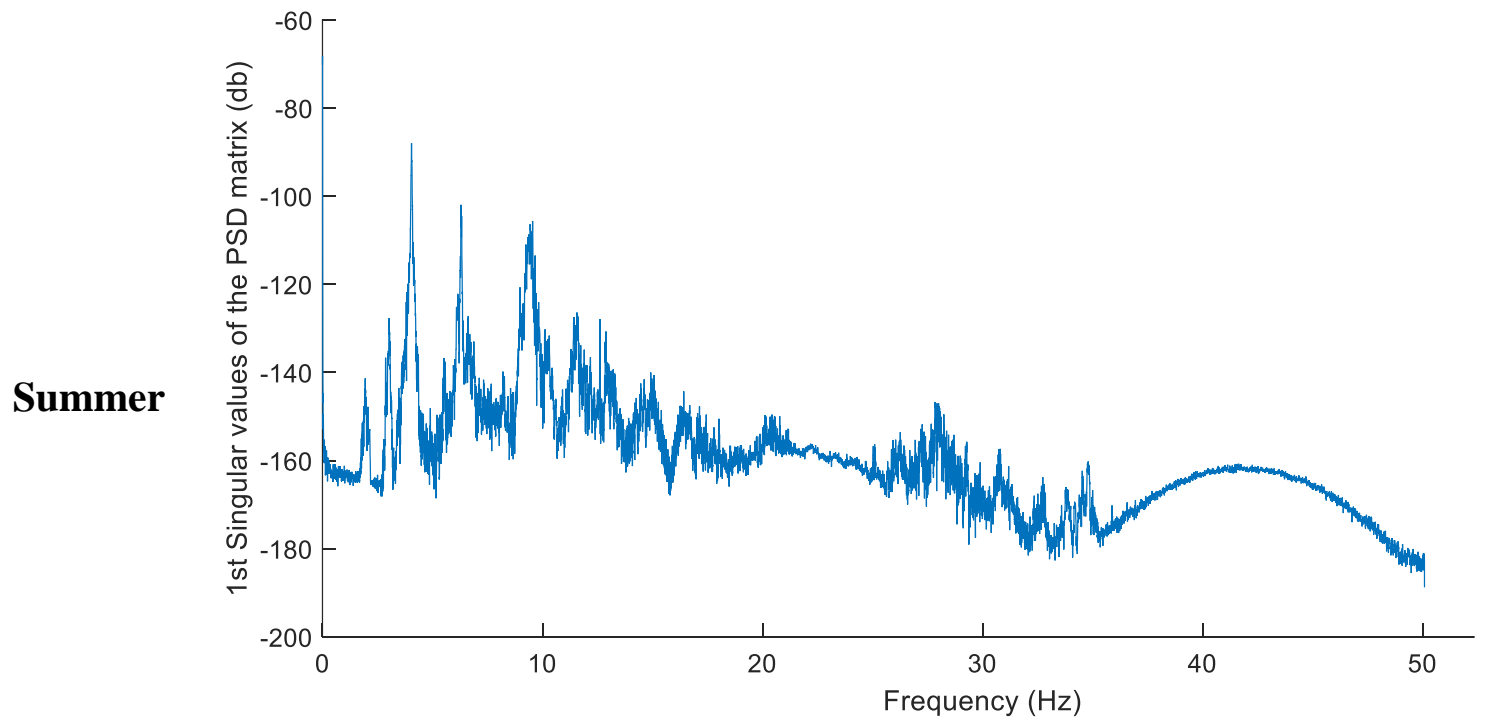

Figure 21. SVD plot of ambient test in summer

The following vertical natural frequencies are picked from the singular values plot. The associated damping ratios and MAC are also estimated and shown below. The MAC values are calculated in MATLAB using the ABRAVIBE (Brandt, 2013) toolbox:

Table 16. Natural frequencies, damping and MAC for summer and winter

\begin{tabular}{ccc|cc|cc}
\hline & $\mathbf{1}^{\text {st }} \boldsymbol{f}_{\boldsymbol{n}}(\mathbf{H z})$ & $\zeta(\%)$ & $\mathbf{2}^{\text {nd }} \boldsymbol{f}_{\boldsymbol{n}}(\mathbf{H z})$ & $\zeta(\%)$ & $\mathbf{3}^{\text {rd }} \boldsymbol{f}_{\boldsymbol{n}}(\mathbf{H z})$ & $\zeta(\%)$ \\
\hline Summer & 4.052 & 0.44 & 6.570 & 0.33 & 9.193 & 0.23 \\
Winter & 4.090 & 1.16 & 5.873 & 1.00 & 11.43 & 0.64 \\
\hline \% Difference $\boldsymbol{f}_{\boldsymbol{n}}$ & 0.90 & & 11.90 & \multicolumn{2}{c}{0.8880} \\
\hline MAC & 0.9935 & 0.9204 & \multicolumn{2}{c|}{0.33} \\
\hline
\end{tabular}

From Table 17 it can be seen that all three modes are quite similar during ambient vibration tests in the summer and winter as seen from a high MAC value. The third mode however has a lower MAC value as provided in Table 16. Overall, the mode shapes correlate quite well between the two times of the year. However, it can also be seen that the percentage difference in the natural frequency estimates has increased from the first to the third natural frequency. This suggests a definite shift in the modal parameters of the system from summer to winter. The effect of temperature has been shown to affect the dynamic characteristics of a bridge (Moorty \& Roeder, 1992). It is also seen that the damping of the structure is also fluctuating showing a decreasing 
trend from the first mode to the third. No conclusive deductions can be outlined from the damping values and it needs to be investigated further in the future.

Table 17 below shows mode shapes obtained from the summer and winter vibration tests:

Table 17. Obtained mode shapes in the summer and winter

Summer
Shape
Mode
Shape
$3^{\text {st }}$
Mode
Shape

The MAC values between the forced and ambient tests conducted around the year (January and July 2016) are also shown in Table 18. It can be seen that the natural frequencies identified through impact hammer test and ambient vibration tests are very close to each other. The third mode MAC is not shown as only a torsional mode was obtained in the forced vibration test as compared to pure bending modes in ambient tests.

Table 18. MAC values for forced and ambient vibration tests

\begin{tabular}{ccc}
\hline & ${\text { sst } \boldsymbol{f}_{\boldsymbol{n}}(\mathbf{H z})}$ 2nd $\boldsymbol{f}_{\boldsymbol{n}}(\mathbf{H z})$ \\
\hline MAC Winter-Forced & 0.9959 & 0.9989 \\
\hline MAC Summer-Forced & 0.9975 & 0.9195 \\
\hline
\end{tabular}


The recorded deck temperatures for each time of the year the tests were performed are provided in Table 19.

Table 19. Deck and ambient temperatures during testing

\begin{tabular}{|c|c|c|c|c|c|}
\hline \multicolumn{3}{|c|}{ Summer } & \multicolumn{3}{|c|}{ Winter } \\
\hline Time & $\begin{array}{c}\text { Deck } \\
\text { Temperature } \\
\left(^{\circ} \mathrm{F}\right)\end{array}$ & $\begin{array}{c}\text { Ambient } \\
\text { Temperature } \\
\left.{ }^{\circ} \mathbf{F}\right)\end{array}$ & Time & $\begin{array}{c}\text { Deck } \\
\text { Temperature } \\
\left.{ }^{\circ} \mathbf{F}\right)\end{array}$ & $\begin{array}{c}\text { Ambient } \\
\text { Temperature } \\
\left({ }^{\circ} \mathbf{F}\right)\end{array}$ \\
\hline 3:00 PM & 106.25 & 82.90 & 12:00 PM & 19.11 & 23.00 \\
\hline 4:00 PM & 101.84 & 82.90 & 1:30 PM & 21.69 & 24.10 \\
\hline 5:15 РМ & 97.25 & 84.00 & 3:30 PM & 21.80 & 26.10 \\
\hline Average & 101.78 & 83.27 & Average & 20.87 & 24.40 \\
\hline
\end{tabular}

It is clearly observed from the table above that the bridge surface temperatures were drastically different (a difference of about $80^{\circ} \mathrm{F}$ ) between the two times of the year. The effect of this temperature change can be clearly observed from the natural frequency shift in Table 16. It has been observed in previous literature that natural frequency values increased for the same mode due to stiffening effects caused by temperature drops (Moser \& Moaveni, 2011; Xia et al., 2011).

A similar behavior is observed for the third mode where the change in natural frequency is an increase of about $24.33 \%$. This is however inconclusive as the second mode shows a decrease in the natural frequency estimate of $11.9 \%$ from the summer to winter. It cannot be suggested from the data that lowered temperature cause a decrease in the natural frequency, however it can be ascertained that temperature effects shift the modal characteristics of the structure.

\subsection{Human Induced Vibration Tests}

Human induced vibration tests in this section are first summarized from each of the design guidelines. This includes calculation of an analytical acceleration limit based on that design guide. This is then followed by the experimentally obtained acceleration values for the bridge. These are then finally compared and serviceability of the structure assessed in the light of different codes. 


\subsubsection{Analytical Results}

Using the AISC steel design guide, the first item to check is the deflection parameter. Since it is likely that the longest span will vibrate the most and register higher acceleration limits, it will be selected for calculations. These are calculated using the equation (47) as follows:

$$
\Delta=\frac{5 w L^{4}}{384 E_{s} I_{t}}=\frac{5(1879 p l f)(70 f t)^{4}\left(1728 i n^{3} / f t^{3}\right)}{384\left(29 \times 10^{6} p s i\right)\left(132749 i n^{4}\right)}=0.2636 \mathrm{in}
$$

Then the fundamental frequency from equation (46) is estimated as:

$$
f_{n}=0.18 \sqrt{\frac{g}{\Delta}}=0.18 \sqrt{\frac{386 \mathrm{in} / \mathrm{s}^{2}}{0.2636 \mathrm{in}}}=6.89 \mathrm{rad} / \mathrm{s}
$$

The total weight of the bridge can be estimated as:

$$
W=w L=1879 \mathrm{plf} * 70 \mathrm{ft}=131530 \text { lbs or } 131.5 \mathrm{kips}
$$

AISC recommends using a damping value of 0.01 for outdoor footbridges. (It will be observed that the experimental damping is also around 0.01) Using this we can then obtain:

$$
\beta W=0.01 * 131.5 \text { kips }=1.32 \text { kips or } 1315.3 \text { lbs }
$$

Now using equation (44) we can assess the resonance response function as:

$$
\frac{a_{p}}{g}=\frac{P_{o} \exp \left(-0.35 f_{n}\right)}{\beta W}=\frac{92 \mathrm{lbs} \exp (-0.35(6.89 \mathrm{rad} / \mathrm{s})}{1315.3 \mathrm{lbs}}=0.627 \% \text { gravity or } 0.06 \mathrm{~m} / \mathrm{s}^{2}
$$

The above is much less than the prescribed limit of $5 \%$ or $0.49 \mathrm{~m} / \mathrm{s}^{2}$ for outdoor footbridges.

Under the AASHTO LRFD design guidelines the following evaluations are done:

$$
\begin{gathered}
f \geq 2.86 \ln \left(\frac{180}{W}\right)=2.86 \ln \left(\frac{180}{357 \text { kips }}\right)=-1.95 \mathrm{~Hz} \\
W \geq 180 e^{-0.35 f_{n}}=180 e^{-0.35 * 4.019}=44.09 \text { kips }
\end{gathered}
$$

It can be observed that with the in-situ bridge weight of 357 kips a negative frequency is obtained which is much below the real lowest vertical frequency of the bridge. Using that low vertical experimental natural frequency, the weight of the bridge is estimated as 44.09 kips which is below the original weight. Therefore, the design criteria are satisfied. 
To estimate the maximum accelerations using HIVOSS guideline, two design situations are considered. The first design situation (D1) corresponds to the daily usage with a traffic class of TC2 and a target comfort level of CL1. The second situation (D2) resembles the case where a high traffic occurs such as during a sporting event (i.e. football weekends). The traffic class assigned to D2 is TC4 and the target comfort level is CL2.

To estimate the maximum accelerations using SÉTRA guideline, the selected footbridge was considered as Class II footbridge. According to Table 7, the dynamic response of the footbridge was calculated under Case 3 loading conditions since its vertical natural frequency falls into Range 3. The damping ratio was assumed to be $0.4 \%$, which is the suggested average value by both HIVOSS and SÉTRA guidelines for the selected footbridge. The SDOF approach was used to calculate the maximum acceleration of the footbridge under each design situation.

Table 20 summarizes the parameters used to calculate the design loads and, the corresponding harmonic loads and predicted maximum acceleration response according to each design case.

Table 20. Analytical results for peak acceleration response

\begin{tabular}{cccccc}
\hline Design code & $d\left(p / \mathrm{m}^{2}\right)$ & $n_{p}$ & $\psi$ & $p(t)\left(\mathrm{N} / \mathrm{m}^{2}\right)$ & $a_{\max }\left(\mathrm{m} / \mathrm{s}^{2}\right)$ \\
\hline HIVOSS - D1 & 0.2 & 4.8 & 0.25 & $p(t)=1.360 \cos (2 \pi(4.019) t)$ & 0.32 \\
HIVOSS - D2 & 1 & 29.1 & 0.25 & $p(t)=8.238 \cos (2 \pi(4.019) t)$ & 1.81 \\
SÉTRA & 0.8 & 9.6 & 1 & $p(t)=2.721 \cos (2 \pi(4.019) t)$ & 0.61 \\
\hline
\end{tabular}

\subsubsection{Experimental Results}

Acceleration time histories in vertical (V) and transverse (T) directions were collected from the performed pedestrian interaction tests as shown before in Figure 11 and Figure 12. In the transverse direction the estimated natural frequencies were below the serviceability design limit and therefore transverse data was ignored. Figure 11 is redrawn to rename the sensors as shown: 


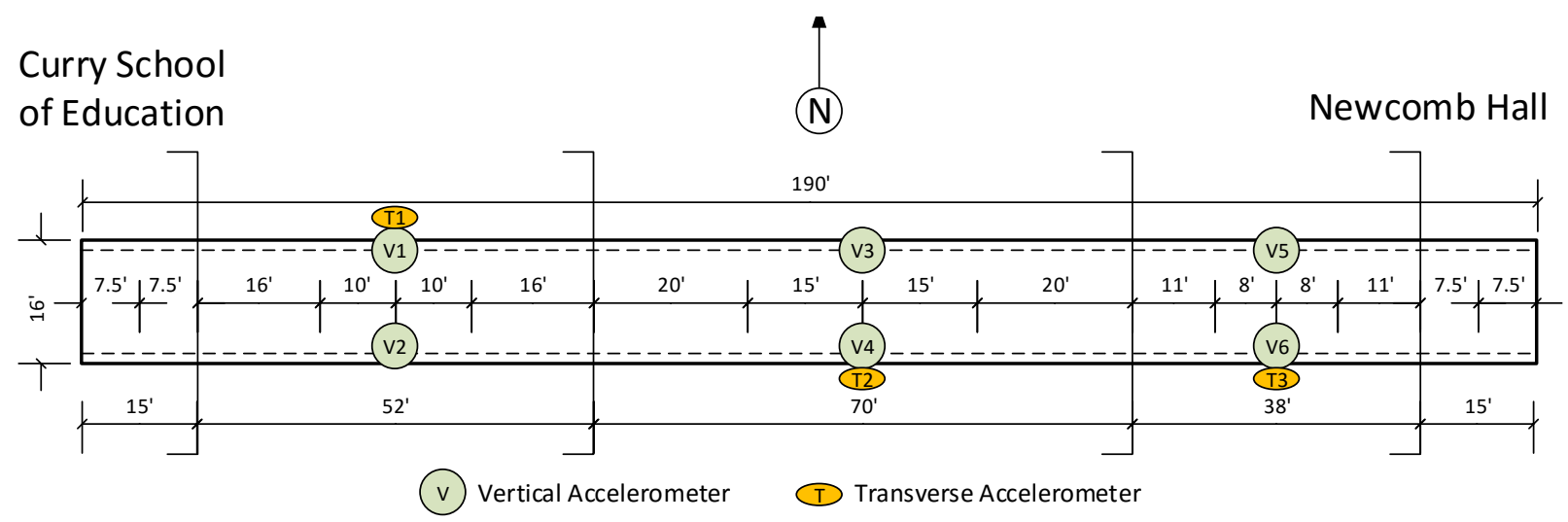

Figure 22. Marked accelerometer locations (regeneration of Figure 11)

For the first serviceability technique the collected data included acceleration records over the course of the tests for all of the four loading scenarios ( 9 people at 3 different walking frequencies and random flow of pedestrians) and their corresponding repetitive runs. Representative sets of raw data collected from the synchronized tests from the accelerometer V3 and T3 are illustrated in Figure 23, which highlights the sensitivity of the bridge dynamic response to the imposed loading frequency. Note that for the pedestrian frequency of $2 \mathrm{~Hz}$, the second harmonic of the step frequency is almost equal to the fundamental frequency of the footbridge in the vertical direction. Therefore, a resonant response was observed in the acceleration history.
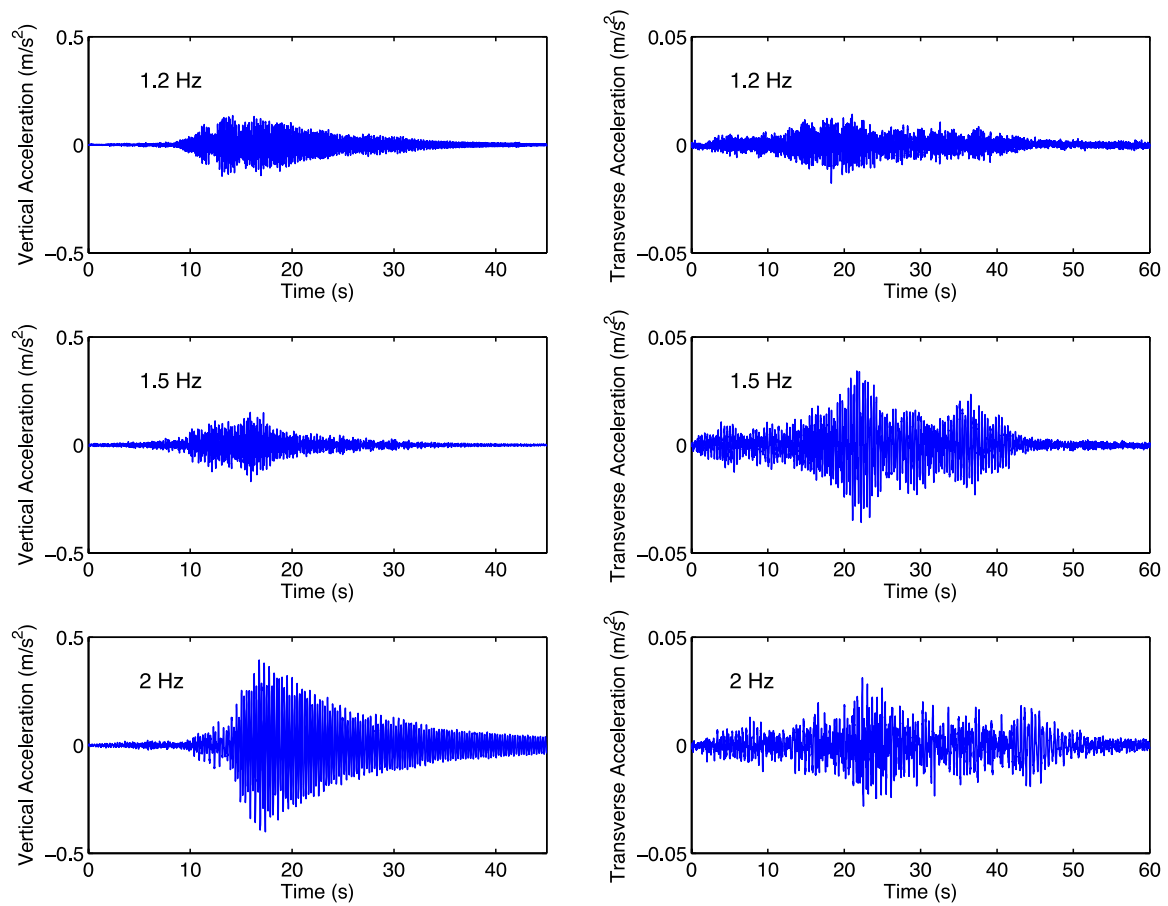

Figure 23. Vertical (V3) and transverse (T3) accelerations due to walk at different frequencies 
The acceleration time histories obtained from the accelerometer V3 during the passage of about 40 people on the footbridge for three different tests are shown in Figure 24. It can be seen that the maximum acceleration (about $0.2 \mathrm{~m} / \mathrm{s} 2$ ) was lower than the peak value obtained during the synchronized tests at $2 \mathrm{~Hz}$.
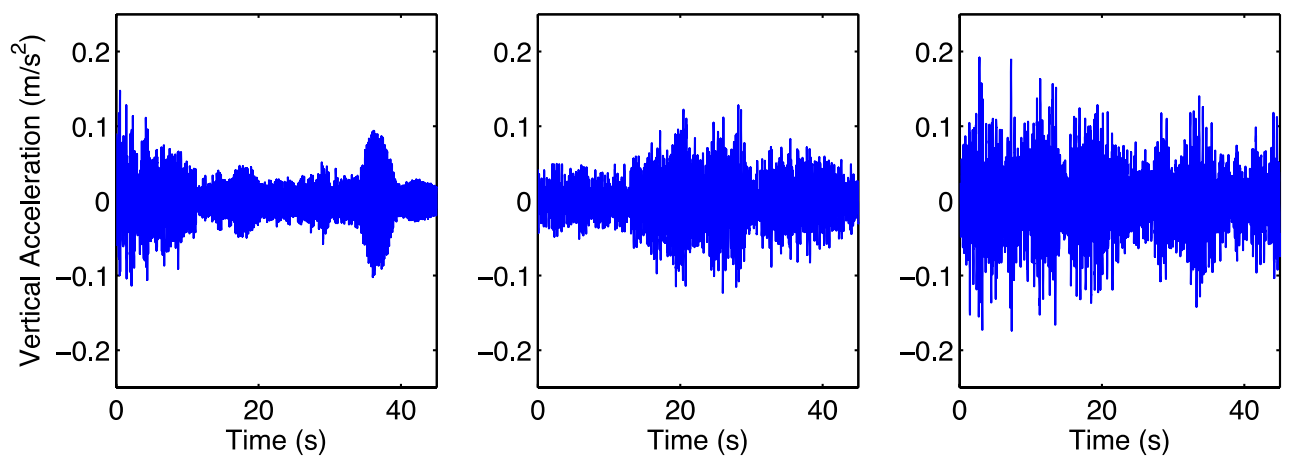

Figure 24. Vertical acceleration time histories at V3 during random flow of pedestrian tests

The raw data was post-processed to define the peak acceleration values obtained from each test run. Table 21 summarized the peak acceleration values at different locations according to the mounted instrumentations. As it is given, the peak acceleration values in both vertical and horizontal directions demonstrate significant variations according to the sensor location. However, the maximum acceleration responses for each direction were highlighted in the table, and used in this study for comparison to the allowable limits provided by current design guidelines.

Table 21. Peak acceleration responses $\left(\mathrm{m} / \mathrm{s}^{2}\right)$

\begin{tabular}{cccccccccc}
\hline Test & V1 & V2 & V3 & V4 & V5 & V6 & T1 & T2 & T3 \\
\hline $\mathbf{1 . 2 ~ H z}$ & 0.098 & 0.097 & 0.145 & $\underline{\mathbf{0 . 1 4 6}}$ & 0.059 & 0.066 & 0.015 & $\underline{\mathbf{0 . 0 2 1}}$ & 0.010 \\
$\mathbf{1 . 5 ~ H z}$ & 0.160 & 0.154 & $\underline{\mathbf{0 . 1 6 9}}$ & 0.153 & 0.119 & 0.100 & 0.033 & $\underline{\mathbf{0 . 0 3 6}}$ & 0.016 \\
$\mathbf{2 ~ H z}$ & 0.207 & 0.200 & $\underline{\mathbf{0 . 4 0 0}}$ & 0.396 & 0.147 & 0.129 & 0.028 & $\underline{\mathbf{0 . 0 3 1}}$ & 0.024 \\
Random & 0.194 & 0.189 & 0.192 & 0.169 & 0.163 & 0.161 & 0.044 & 0.046 & 0.028 \\
\hline
\end{tabular}

In the second serviceability testing approach, acceleration data was recorded for the case of one pedestrian jumping at a frequency of $2 \mathrm{~Hz}$. Two extra vertical sensors were placed on one of the end spans. The testing plan as shown in Figure 12 is redrawn in Figure 25 below to account for the naming of the sensors. In this part serviceability assessment was performed by pedestrian jumping motion at each of the mid points of the three major spans. 


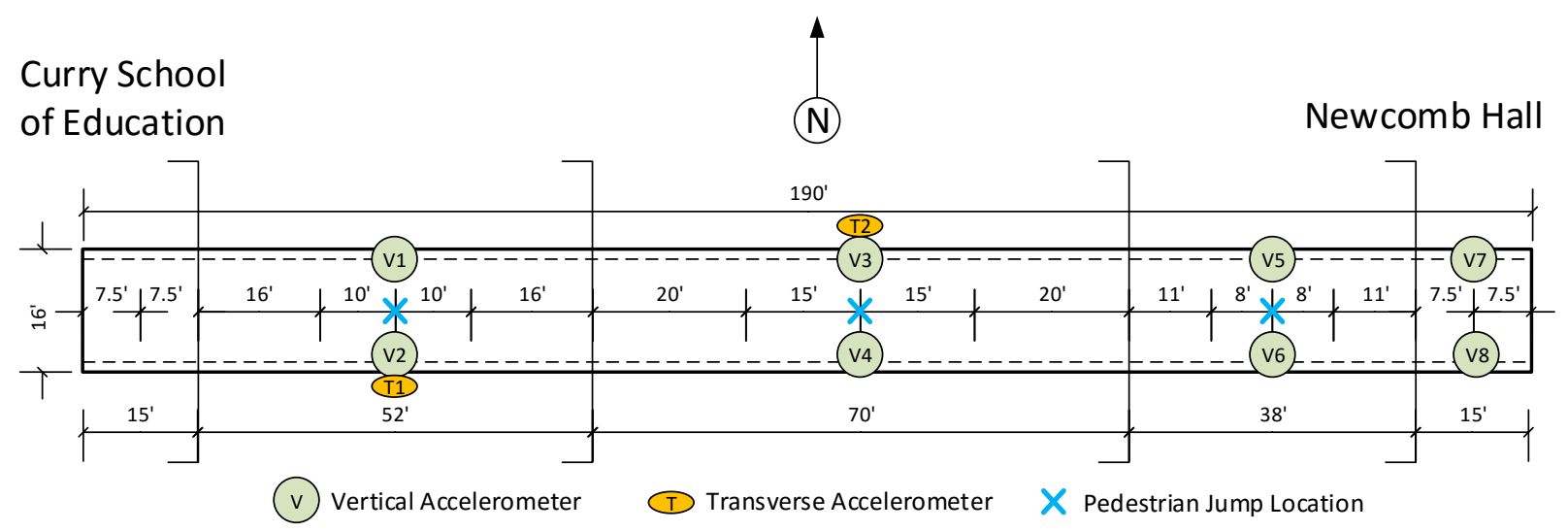

Figure 25. Marked accelerometer locations (regeneration of Figure 12)

Representative sets of raw data collected in the tests from the accelerometer V1, V4 and V6 are illustrated in Figure 26:
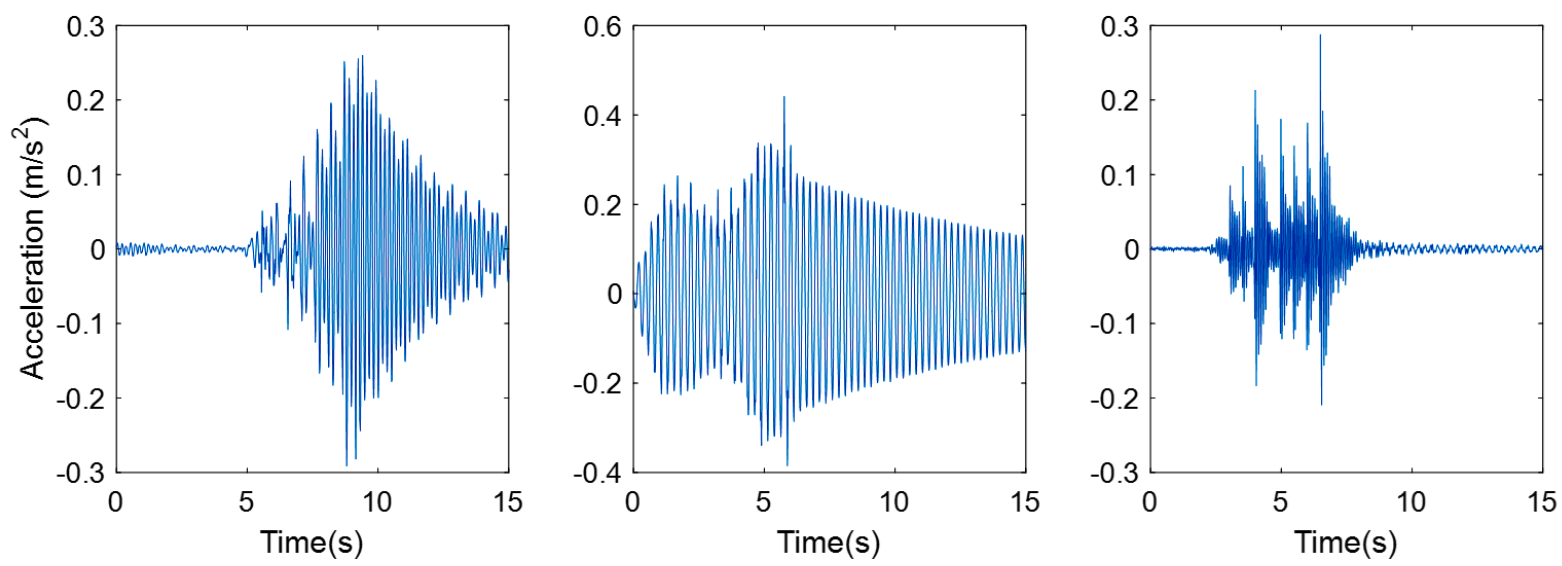

Figure 26. Vertical time histories of V1, V4 and V6 sensors during a jumping motion

Similar to the pedestrian tests discussed above, maximum accelerations were obtained from the raw data for each of the jump test cases. Peak acceleration results for a jumping frequency of $2 \mathrm{~Hz}$ on all three spans are summarized as follows:

Table 22. Peak accelerations for jumping at three different spans $\left(\mathrm{m} / \mathrm{s}^{2}\right)$

\begin{tabular}{lcccccccccc}
\hline & V1 & V2 & V3 & V4 & V5 & V6 & V7 & V8 & T1 & T2 \\
\hline Curry & $\underline{\mathbf{0 . 2 9 1}}$ & 0.276 & 0.236 & 0.213 & 0.021 & 0.030 & 0.011 & 0.009 & 0.020 & 0.010 \\
Center & 0.090 & 0.097 & $\underline{\mathbf{0 . 4 4 3}}$ & 0.441 & 0.035 & 0.045 & 0.014 & 0.014 & 0.004 & 0.020 \\
Newcomb & 0.027 & 0.029 & 0.078 & 0.081 & 0.286 & $\underline{\mathbf{0 . 2 8 8}}$ & 0.065 & 0.101 & 0.007 & 0.008 \\
\hline
\end{tabular}




\subsubsection{Serviceability Evaluation}

The analytical dynamic response together with the critical maximum values of the recorded accelerations in the vertical direction were compared to the limits provided in current guidelines.

Figure 27 illustrates the comparison between critical acceleration values obtained from experimental and analytical approaches, to the limits provided in current guidelines. As demonstrated, the maximum critical acceleration response $\left(0.40 \mathrm{~m} / \mathrm{s}^{2}\right)$ obtained from the pedestrian interaction tests, satisfy the limits of the codes, which would ensure the vibration serviceability of the selected structure. According to AISC guidelines the maximum limit for peak acceleration for comfort is $0.49 \mathrm{~m} / \mathrm{s}^{2}$ and which is greater than our maximum critical acceleration response from both the analytically estimated $\left(0.06 \mathrm{~m} / \mathrm{s}^{2}\right)$ and experimentally obtained value $\left(0.44 \mathrm{~m} / \mathrm{s}^{2}\right)$.

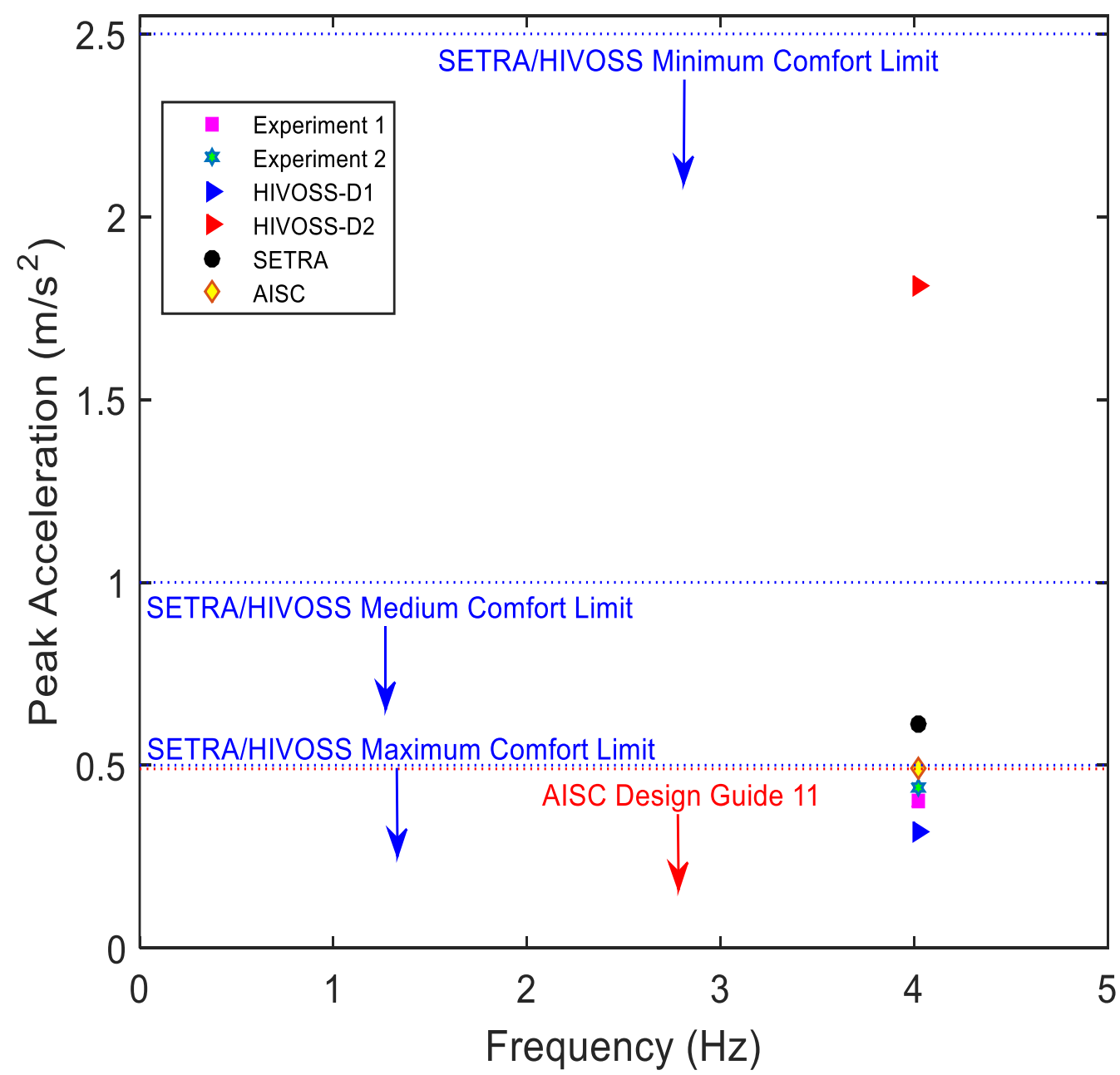

Figure 27. Evaluation of pedestrian-induced dynamic response 
The peak acceleration $\left(0.32 \mathrm{~m} / \mathrm{s}^{2}\right)$ estimated for the design situation D1 according to HIVOSS guideline is also below the target comfort level (maximum comfort or $a_{\max }<0.50 \mathrm{~m} / \mathrm{s}^{2}$ ) selected for this situation. However, the analytical values $\left(1.81 \mathrm{~m} / \mathrm{s}^{2}\right)$ for critical dynamic responses of the footbridge in the design situation D2 exceed the target comfort limit (medium comfort or $\left.a_{\max }<1 \mathrm{~m} / \mathrm{s}^{2}\right)$. The peak acceleration $\left(0.61 \mathrm{~m} / \mathrm{s}^{2}\right)$ estimated by SÉTRA guideline indicates a medium comfort level for the footbridge.

Note that the selected number of people for the pedestrian interaction tests corresponds to an equivalent pedestrian density of $0.7 \mathrm{p} / \mathrm{m}^{2}$ for this footbridge. The SÉTRA guidelines classifies the bridge as Class III for such a traffic level and do not require a dynamic analysis for that footbridge. For a pedestrian density $d=0.7 \mathrm{p} / \mathrm{m}^{2}$, the HIVOSS guidelines predicts the peak acceleration as $0.58 \mathrm{~m} / \mathrm{s}^{2}$. This value overestimates the measured peak accelerations from the experimental tests. For this bridge, it can be concluded that the analytical expressions provided in HIVOSS and SÉTRA guidelines tend to be conservative for estimating the peak acceleration response of the studied footbridge. Nevertheless, they are considered as safe margins (upper bound on the behavior) that can help engineers control the vibration vulnerability of the system. 


\section{CONCLUSIONS}

In this research project a multi-span steel girder pedestrian bridge with excessively lively behavior was investigated to obtain key information about the structure's dynamic characteristics and to examine that behavior through a comparative vibration serviceability analysis. In the initial phase of the research project, a preliminary finite element model was developed and the resulting natural frequencies and mode shapes were recorded. These numerical dynamic properties then acted as a baseline for the actual field tests on the pedestrian bridge. Three different types of acceleration time data were collected from the sensors on the bridge. The first type of data resulted from a forced vibration test where an impact hammer was used to excite the structure at certain locations and the response recorded. The second type of data was collected from an ambient test where the bridge was allowed to be in service and the data recorded for an extended period of time. The third type of data was collected from a series of pedestrian walking and jumping tests at certain frequencies in effort to excite the bridge's critical limits.

Results from the impact hammer and ambient data were found to correlate very well resulting in a high modal assurance criteria value of around 0.98. Since ambient tests were conducted in two different times of the year, it was observed that the bridge's dynamic characteristics changed considerably from summer to winter. This was attributed to the change in stiffness parameters of the bridge due to temperature changes. In the pedestrian loading tests, it was observed that the vertical natural frequencies were below the limit in the design codes HIVOSS and SÉTRA and therefore acceleration records were obtained. Peak acceleration response was recorded at the midpoint of the mid-span at a walking frequency of $2 \mathrm{~Hz}$. This was used to conduct another serviceability test with just one person jumping at this critical frequency and the peak acceleration response was recorded at $0.44 \mathrm{~m} / \mathrm{s}^{2}$. These were compared with the limiting values in the design codes and were found to be within the prescribed range. Numerically estimated values in the design codes were also compared to the limits and it was found that in the HIVOSS design situation of D2, the medium comfort level was exceeded.

It could be safely concluded that the pedestrian bridge does not have any serious serviceability issues and only some design situations may result in a decreased comfort level. It is however recommended that further design classes be evaluated according to the code and results compared to the finite element model along with the experimental data. 


\section{FUTURE WORK}

Based on the obtained results, it can be seen that the serviceability assessment of the Ruffner footbridge is satisfied according to the different design standards. However, there are still several items that will be accomplished in the future. These include but are not limited to:

1) Use of other data processing and modal identification techniques. These could include time or frequency domain based identification methods such as SSI-COV (Maia \& Silva, 2014). These data processing techniques would also improve the damping estimation. Since EFDD is not the most robust predictor of damping, other different algorithms can be tried to estimate a more accurate damping ratio.

2) Improved processing parameters. These may include items such as filtering, windowing and leakage effects. Data capturing parameters such as sampling and time stamp could be improved to obtain highest quality of data.

3) Model updating procedure. Dynamic data can be used to update the finite element model and to correlate the data exactly with the numerical model. The model can also be tested for numerical simulations of pedestrian loadings. Different pedestrian motion configurations can be simulated in the model and response obtained.

4) Increased pedestrian interaction test configurations. Experimental tests did not consist of all the pedestrian motion configurations or traffic classes. Therefore, this could be implemented to ensure that the maximum accelerations under service are obtained. These critical configurations could then be used to create a maximum acceleration testing plan. Results can be further analyzed for a more robust serviceability assessment.

5) A more rigorous comparative assessment using other guidelines.

In general, the topic of vibration serviceability is still relatively new and research in the United States on the subject has not been as extensive as in Europe. Incorporation of the pedestrian walking harmonics into the design code is not quite clear and no clear guidance as to how many harmonics should be incorporated in design, is present in the guides. Damping has been briefly investigated in this research, but with more refined algorithms it can be more accurately estimated. The design codes must also be expanded to integrate all types of pedestrian structures instead of just simple concrete or steel bridges. It is recommended that the AISC design codes be expanded and vibration serviceability explored further in the future. 


\section{REFERENCES}

AASHTO. (2009). LRFD Guide Specifications for the Design of Pedestrian Bridges (Vol. Attachment).

Abdel-Ghaffar, A., \& Scalan, R. (1985). Ambient Vibration Studies of Golden Gate. Journal of Engineering Mechanics, 111(4), 463-482.

Bachmann, H., \& Ammann, W. (1987). Vibrations in structures: induced by man and machines (3e ed.). IABSE.

Bacinskas, D., Kamaitis, Z., Jatulis, D., \& Kilikevicius, A. (2014). Field load testing and structural evaluation of steel truss footbridge. In The 9th International Conference "Environmental Engineering. ”Vilnius, Lithuania.

Bačinskas, D., Kamaitis, Z., \& Kilikevičius, A. (2013). 935 . A sensor instrumentation method for dynamic monitoring of railway bridges. Journal of Vibroengineering, 15(1), 176-185.

Blanchard, J., Davies, B. L., \& Smith, J. W. (1977). Design criteria and analysis for dynamic loading of footbridges. In Proceeding of a Symposium on Dynamic Behaviour of Bridges at the Transport and Road Research Laboratory. Crowthorne, Berkshire, England: Transport and Road Research Laboratory.

Brandt, A. (2011). Noise and Vibration Analysis: Signal Analysis and Experimental Procedures (First). John Wiley \& Sons.

Brandt, A. (2013). ABRAVIBE “A MATLAB/Octave toolbox for Noise and Vibration Analysis and Teaching." Department of Technology and Innovation, University of Southern Denmark.

Brincker, R., Ventura, C. E., \& Anderson, P. (2001). Damping Estimation by Frequency Domain Decomposition. In Proceedings of IMAC 19: A Conference on Structural Dynamics (pp. 698703). Orlando: Society for Experimental Mechanics.

Brincker, R., Zhang, L., \& Andersen, P. (2000). Modal Identification from Ambient Responses using Frequency Domain Decomposition. In Proceedings of the 18th International Modal Analysis Conference (pp. 625-630). 
Brownjohn, J. M. W., Moyo, P., Omenzetter, P., \& Lu, Y. (2003). Assessment of Highway Bridge Upgrading by Dynamic Testing and Finite-Element Model Updating. Journal of Bridge Engineering, 8(3), 162-172. http://doi.org/10.1061/(ASCE)1084-0702(2003)8:3(162)

Caifeng, W., Lei, J., Shiqiao, G., \& Linxiong, C. (2015). Ambient Vibration Survey and Vibration Serviceability Evaluation on Footbridges. In International Conference on Chemical, Material and Food Engineering (CMFE-2015) (pp. 545-549).

Chopra, A. K. (2012). Dynamics of Structures. (H. Stark, Ed.) (Fourth, Vol. 1). NJ, USA: PrenticeHall. http://doi.org/10.1017/CBO9781107415324.004

CSI. (2013). SAP2000 integrated structural analysis \& design software. Berkeley, California: CSI Inc.

Cunha, A., \& Caetano, E. (2005). From input-output to output-only modal identification of civil engineering structures. In 1st International Operational Modal Analysis Conference, IOMAC 2005 (pp. 1-22).

Cunha, A., Caetano, E., Magalhães, F., \& Moutinho, C. (2012). Recent perspectives in dynamic testing and monitoring of bridges. Structural Control and Health Monitoring, 20 (August 2012), 852-876. http://doi.org/10.1002/stc

da Silva, J. G. S., Vellasco, P. C. G. d S., de Andrade, S. A. L., de Lima, L. R. O., \& Figueiredo, F. P. (2007). Vibration analysis of footbridges due to vertical human loads. Computers and Structures, 85(21-22), 1693-1703. http://doi.org/10.1016/j.compstruc.2007.02.012

Dallard, P., Fitzpatrick, T., Flint, A., Low, A., Smith, R. R., Willford, M., \& Roche, M. (2001). London Millennium Bridge: Pedestrian-Induced Lateral Vibration. Journal of Bridge Engineering, 6(December), 412-417.

Dey, P., Walbridge, S., \& Narasimhan, S. (2015). Vibration Serviceability Analysis of Aluminum Pedestrian Bridges Subjected to Crowd Loading. In 6th International Conference on Advances in Experimental Structural Engineering.

El-robaa, A., Gaawan, S., \& Malek, C. (2015). Evaluation of human-induced vibration of 
continuous footbridges. MATEC Web of Conferences, 24, 07002.

Gheitasi, A., Ozbulut, O. E., Usmani, S., Alipour, M., \& Harris, D. K. (2016). Vibration Serviceability Assessment of An In-service Pedestrian Bridge Under Human-Induced Excitations. In Proceedings of the IMAC-XXXIV. Orlando: Society for Experimental Mechanics.

Gismart [Mobile application software]. (2016). Real Metronome Free. Apple.

Gudmundsson, G. V, Ingolfsson, E. T., Einarsson, B., \& Bessason, B. (2008). Serviceability assessment of three lively footbridges in reykjavik. In Third International Conference Footbridge 2008: Footbridges for Urban Renewal.

Halling, M. W., Ball, A., Esplin, R., \& Hsieh, K.-H. (2004). Modal Analysis and Modeling of Highway Bridges. In 13th World Conference on Earthquake Engineering (p. 2996). Vancouver, B.C, Canada.

Harris, D., Ozbulut, O., Alipour, M., Usmani, S., \& Kassner, B. (2015). Implications of Load Rating Bridges in Virginia with Limited Design or As-Built Details. In 7th International Conference on Structural Health Monitoring of Intelligent Infrastructure. Torino, Italy.

Hauksson, F. (2005). Dynamic behaviour of footbridges subjected to pedestrian-induced vibrations. Master's thesis. Lund University.

Heinemeyer, C., Butz, C., Keil, A., Schlaich, M., Goldack, A., Trometor, S., .. Caetano, E. (2009). Design of Leightweight footbridges for human induced vibrations (Vol. 3).

HIVOSS. (2007). Human induced Vibrations of Steel Structures Design of Footbridges Background Document.

HIVOSS. (2008). Human induced Vibrations of Steel Structures Design of Footbridges Guideline.

Honda, H. (2012). Vibration Serviceability of Bongossi Wood. In P. Quenneville (Ed.), World Conference on Timber Engineering (WCTE) (p. 5). Auckland, NZ.

Irvine, T. (2000). An Introduction To Frequency Response Functions. 
Maia, N. M. M., \& Silva, J. M. M. (2014). Modal analysis identification techniques. Philosophical Transactions of the Royal Society A Mathematical, Physical and Engineering Sciences, 359(1778), 29-40. http://doi.org/10.1098/rsta.2000.0712

MATLAB. (2015). Natick, MA: MathWorks Inc.

Mola, E., Paksoy, M. A., Rebecchi, G., Busca, G., Scaccabarozzi, M., \& Berardengo, M. (2015). Dynamics of Civil Structures, Volume 2: Proceedings of the 33rd IMAC, A Conference and Exposition on Structural Dynamics, 2015. In J. Caicedo \& S. Pakzad (Eds.), (pp. 157-170). Cham: Springer International Publishing. http://doi.org/10.1007/978-3-319-15248-6_39

Moorty, S., \& Roeder, C. W. (1992). Temperature-dependent bridge movements. Journal of Structural Engineering, 118(4), 1090-1105.

Moser, P., \& Moaveni, B. (2011). Environmental effects on the identified natural frequencies of the Dowling Hall Footbridge. Mechanical Systems and Signal Processing, 25(7), 2336-2357. http://doi.org/http://dx.doi.org/10.1016/j.ymssp.2011.03.005

Murray, T. M., Allen, D. E., \& Ungar, E. E. (2003). Floor vibrations due to human activity. In Steel Design Guide Series 11 (Vol. D811). American Institute of Steel Construction.

Olshausen, B. a. (2000). Aliasing. PSC 129 - Sensory Processes.

PCB.com. (2016). Introduction to Impact Hammers.

Rahmatalla, S., Hudson, K., Liu, Y., \& Eun, H. C. (2014). Finite element modal analysis and vibration-waveforms in health inspection of old bridges. Finite Elements in Analysis and Design, 78, 40-46. http://doi.org/10.1016/j.finel.2013.09.006

Richardson, M. H. (1997). Is it a mode shape, or an operating deflection shape? Sound and Vibration, (30th Anniversary Issue), 1-8.

Rocklin, G. T., Crowley, J., \& Vold, H. (1985). A comparison of H1, H2, and Hv frequency response functions. In Proceedings of the 3rd international Modal Analysis Conference (Vol. 1). Orlando: IMAC. 
Rogers, K. (1997). The Tacoma Narrows Bridge Disaster.

Sadeghi, F., \& Kueh, A. H. (2015). Serviceability Assessment of Composite Footbridge Under Human Walking and Running Loads. Jurnal Teknologi, 4(74), 73-77.

Salgado, R., Branco, J. M., Cruz, P. J. S., \& Ayala, G. (2014). Serviceability assessment of the Góis footbridge using vibration monitoring. Case Studies in Nondestructive Testing and Evaluation, 2(1), 71-76. http://doi.org/10.1016/j.csndt.2014.10.001

Schwarz, B. J., \& Richardson, M. H. (1999). Experimental Modal Analysis. In CSI Reliability Week (Vol. 35, pp. 1-12). Jamestown, California.

SÉTRA. (2006). Technical guide Footbridges Assessment of vibrational behaviour of footbridges under pedestrian loading.

Štimac Grandić, I. (2015). Serviceability verification of pedestrian bridges under pedestrian loading. Tehnicki Vjesnik - Technical Gazette, 22(2), 527-537. http://doi.org/10.17559/TV20131030105641

Van Nimmen, K., Van Den, Gezels, B., \& De Roeck, G. (2010). Vibration serviceability of footbridges : a comparative study of 3 design methodologies. In Proceedings of ISMA2010 International Conference on Noise and Vibration (pp. 1491-1506). KU Leuven.

Wang, X., Kangas, S., Padur, D., Liu, L., Swanson, J. a., Helmicki, A. J., \& Hunt, V. J. (2005). Overview of a Modal-Based Condition Assessment Procedure. Journal of Bridge Engineering, 10(4), 460-467. http://doi.org/10.1061/(ASCE)1084-0702(2005)10:4(460)

Whelan, M. J., Gangone, M. V., Janoyan, K. D., \& Jha, R. (2009). Real-time wireless vibration monitoring for operational modal analysis of an integral abutment highway bridge. Engineering Structures, 31(10), 2224-2235. http://doi.org/10.1016/j.engstruct.2009.03.022

Win-STS. (2011). Boulder, Colorado: Bridge Diagnostics Inc.

Xia, Y., Weng, S., Su, J., \& Xu, Y. (2011). Temperature effect on variation of structural frequencies: from laboratory testing to field monitoring. In The 6th International Workshop 
on Advanced Smart Materials and Smart Structures Technology (pp. 1-10).

Yao, S., Wright, J. R., Pavic, A., \& Reynolds, P. (2002). Forces generated when bouncing or jumping on a flexible structure. In Proceedings of the International Conference on Noise and Vibration (Vol. 2, pp. 563-572).

Živanović, S., Pavic, A., \& Reynolds, P. (2005). Vibration serviceability of footbridges under human-induced excitation: A literature review. Journal of Sound and Vibration, 279(1-2), 174. http://doi.org/10.1016/j.jsv.2004.01.019

Živanović, S., Pavic, A., \& Reynolds, P. (2006). Modal testing and FE model tuning of a lively footbridge structure. Engineering $\quad$ Structures, 28(6), 857-868. http://doi.org/10.1016/j.engstruct.2005.10.012 


\section{APPENDIX I (Experimental Modal Analysis Code)}

Code for forced impact hammer test. The damping part of the code is written by Muhammad M. Sherif (PhD candidate) at University of Virginia:

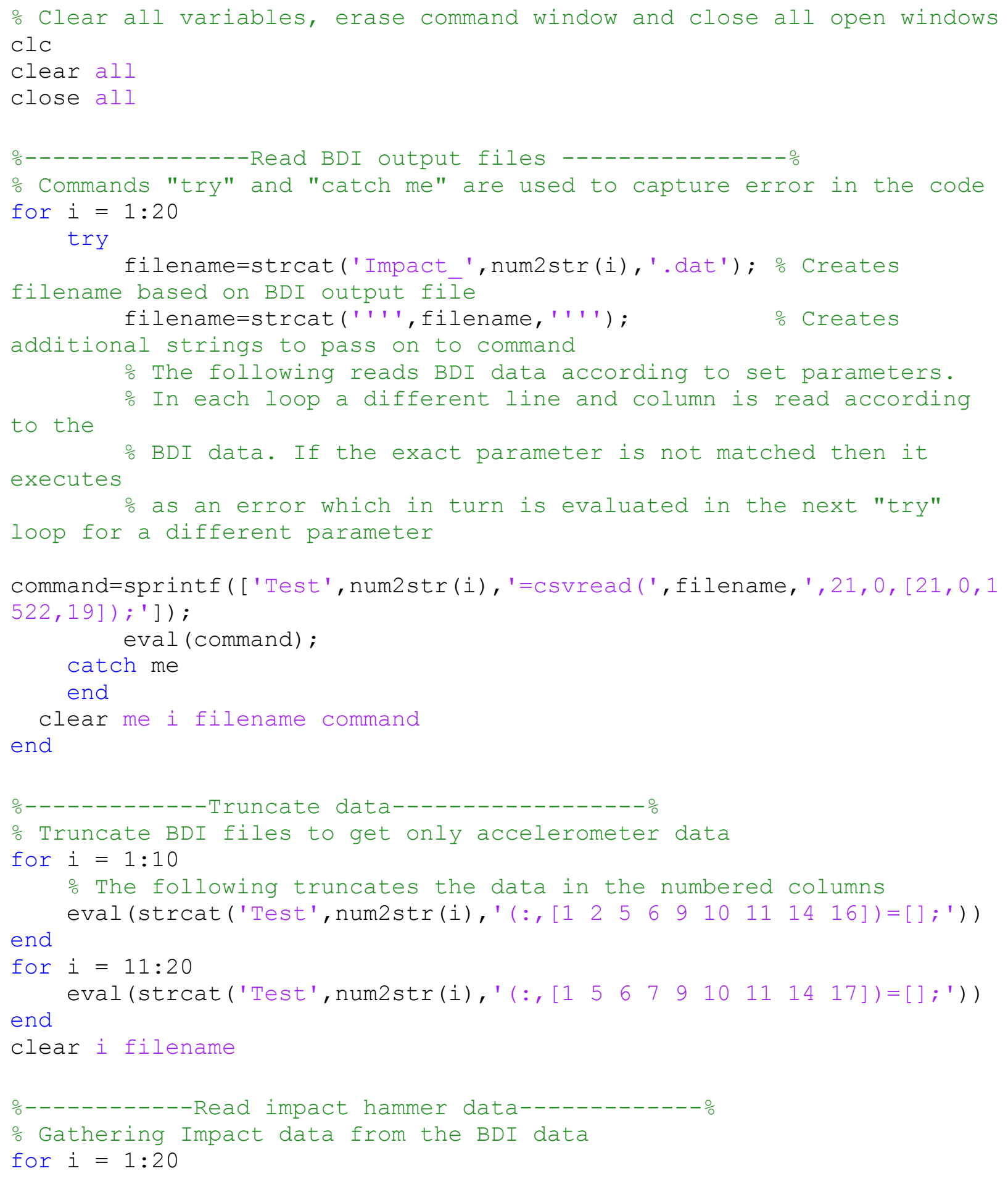




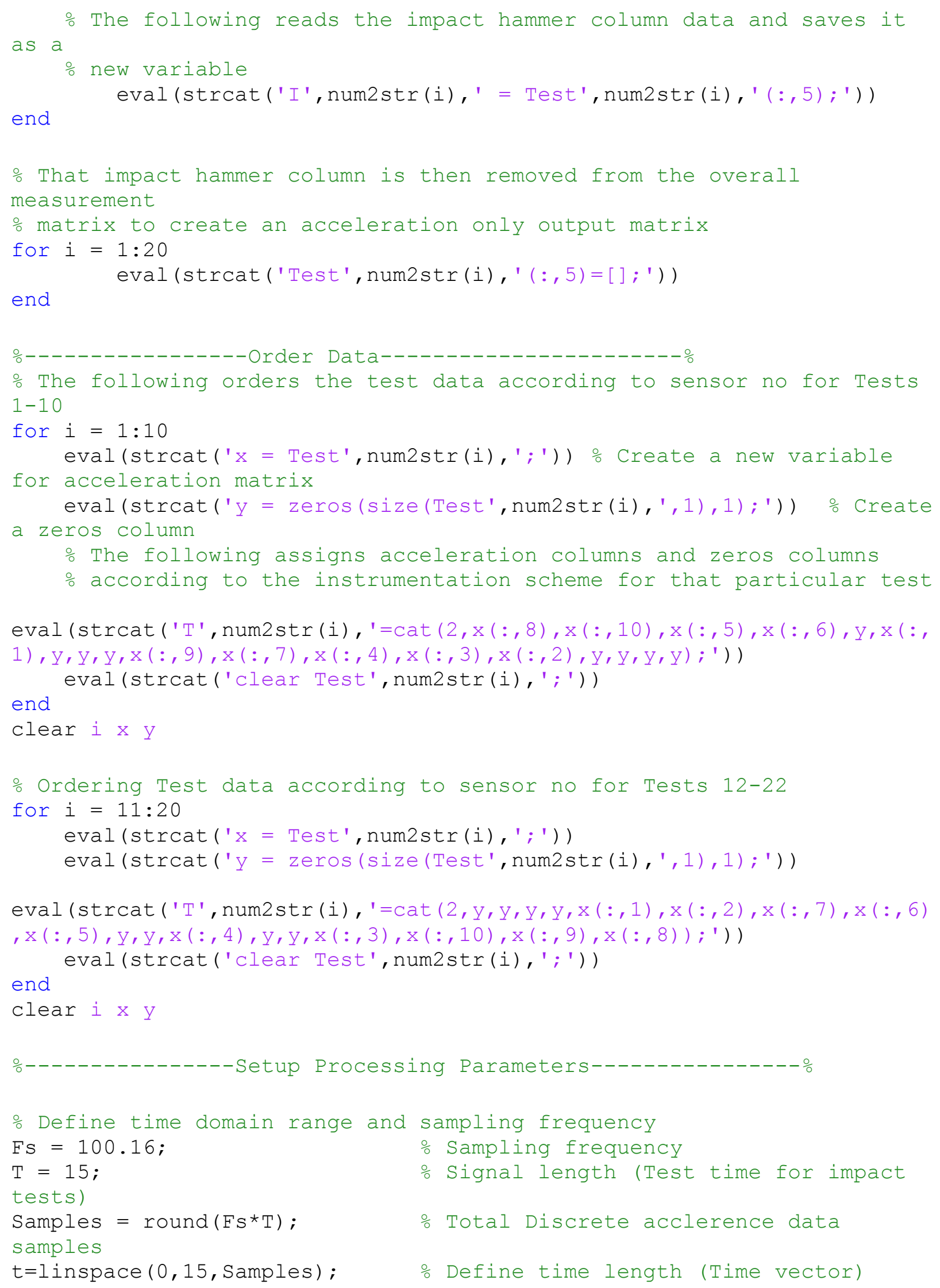


$\mathrm{L}=\operatorname{length}(\mathrm{T} 1(:, 1))$;

N NFT = [ ];

Cooley-Tukey. For default use []

$\therefore$ window $=[]$;

Can use hann(L) or rectwin etc.

$\mathrm{NFFT}=2^{\wedge}$ nextpow2 (L);

window $=$ hann $(\mathrm{L})$;
- Length of data points

\% Number of bins use $2^{\wedge}$ nextpow(L) for [ ]

\% Define window to be used in pwelch.

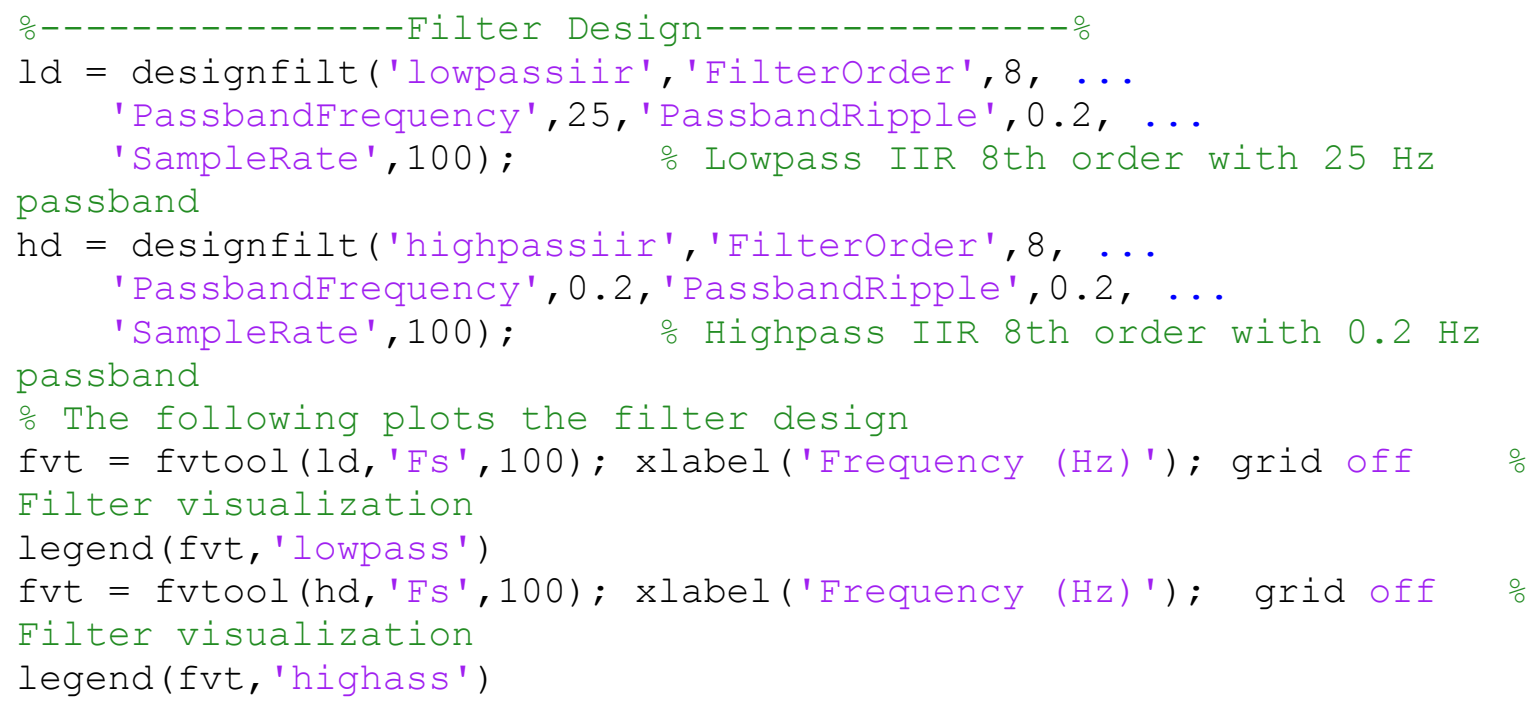

eval (strcat ('[Pff', num2str(i), ',w', num2str(i), '] =pwelch(I', num2str(i), ',window, [],NFFT, FS);')); autospectrum of the impact eval (strcat ('PFF $=P F F+P f f ', \operatorname{num} 2 \operatorname{str}(i), ' ; ')$ ) eval (strcat ('W $W+W$ ', $\operatorname{num} 2 \operatorname{str}(i), ' ; ')$ ) 


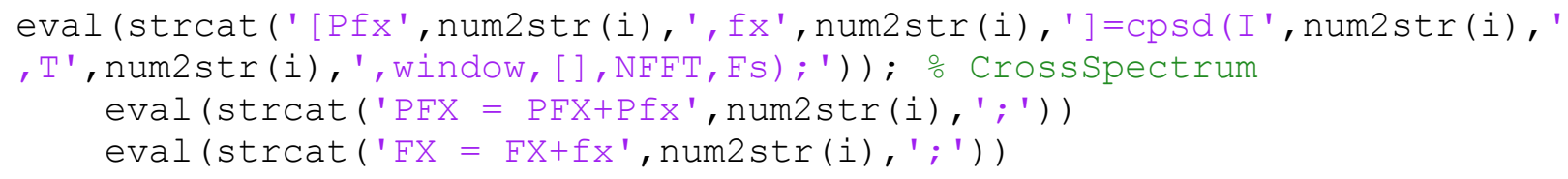

eval (strcat ('[Pxf', num2str(i), ', xf' ', num2str(i), '] =cpsd(T', num2str(i),' , I', num2str(i), ', window, [], NFFT, Fs); ')); \% Crossspectrum eval (strcat ('PXF = PXF+Pxf', num2str(i), ';')) eval (strcat ('XF $=X F+x f ', \operatorname{num} 2 \operatorname{str}(i), ' ; ')$ )

o This part processes only the filtered data eval (strcat ('TF', num2str(i), '=filtfilt(ld, T', num2str(i), ') ;')) \%

Apply the low pass part of the filter to the outputs eval (strcat ('IF', num2str(i), '=filtfilt(ld, I', num2str(i), ') ; ')) \% Apply the low pass part of the filter to the intput eval (strcat ('TF', num2str(i), '=filtfilt (hd, $T F$ ', num2str(i), '); ')) \% Apply the high pass part of the filter to the already low filtered data eval (strcat ('IF', num2str(i), '=filtfilt(hd,IF', num2str(i), ');')) \% Apply the high pass part of the filter to the already low filtered data

eval (strcat (' [Pxxf', num2str(i), ', ff', num2str(i), '] =pwelch (TF', num2str( i), ', window, [], NFFT, FS); ')); \% autospectrum of the response eval (strcat ('PXXf = PXXf+Pxxf', num2str(i), ';')) eval (strcat ('Ff = Fftff', num2str(i), ';'))

eval (strcat (' [Pfff', num2str(i), ', wf', num2str(i), '] =pwelch (IF', num2str( i), ', window, [], NFFT, FS); ')); $\frac{\circ}{0}$ autospectrum of the impact eval (strcat ('PFFf = PFFf+Pfff', $\left.\operatorname{num} 2 \operatorname{str}(i),{ }^{\prime} ; '\right)$ ) eval (strcat ('Wf $=W f+w f ', \operatorname{num} 2 \operatorname{str}(i), ' ; ')$ )

eval (strcat ('[Pfxf', num2str(i), ', fxf', num2str(i), '] =cpsd (IF', num2str(i ) , ', TF', num2str(i), ', window, [ ], NFFT, Fs); ')) ; \% CrossSpectrum eval (strcat ('PFXf = PFXf+Pfxf', num2str(i), ';')) eval (strcat ('FXf = FXftexf', num2str(i),';'))

eval (strcat ('[Pxff', num2str(i), ', xff', num2str(i), '] =cpsd(TF', num2str(i ), ', IF', num2str(i), ', window, [],NFFT, Fs); ')) ; Cross Spectrum eval (strcat ('PXFf = PXFf+Pxff', num2str(i), ';')) eval (strcat ('XFf $=X F f+x f f ', \operatorname{num} 2 \operatorname{str}(i), ' ; ')$ )

end 
\% All the saved variables are averaged for the number of times the

test was

\% conducted. Matrix Sff is repeated to correspond to each sensor

seperately

\% for further processing

$\mathrm{SxX}=\mathrm{PXX} / 5 ; \quad \mathrm{Sfx}=\mathrm{PFX} / 5 ; \quad \mathrm{Sxf}=\mathrm{PXF} / 5 ; \quad \mathrm{Sff}=\mathrm{PFF} / 5$;

Sff=repmat (Sff, 1,18); $\quad$ Spectral Densities (Unfiltered)

$\mathrm{F}=\mathrm{F} / 5 ; \quad \mathrm{W}=\mathrm{W} / 5 ; \quad \mathrm{FX}=\mathrm{FX} / 5 ; \quad \mathrm{XF}=\mathrm{XF} / 5 ;$

$\mathrm{H} 1=$ Sfx./Sff; $\quad \mathrm{H} 2=\mathrm{Sxx} . /$ Sxf; $\quad$ Coh $=\mathrm{H} 1 . / \mathrm{H} 2 ; \div$ Computes the

H1 and H2 frfs and then their coherence

$\mathrm{H} 1($ isnan $(\mathrm{H} 1))=0 ; \quad \mathrm{H} 2($ isnan $(\mathrm{H} 2))=0 ; \quad \operatorname{Coh}($ isnan $(\mathrm{Coh}))=0 ; \quad$ Elements

with divisibility by zero that came up as NaN are changed to 0

Sxxf=PXXf/5; Sfxf=PFXf/5; Sxff=PXFf/5; Sfff=PFFf/5;

Sfff=repmat (Sfff, 1,18); 응 Spectral Densities (Filtered)

$\mathrm{Ff}=\mathrm{Ff} / 5 ; \quad \mathrm{Wf}=\mathrm{Wf} / 5 ; \quad \mathrm{FXf}=\mathrm{FXf} / 5 ; \quad \mathrm{XFf=XFf/5;}$

H1f = Sfxf./Sfff; $\quad H 2 f=$ Sxxf./Sxff; $\quad$ Cohf $=$ H1f./H2f;

H1f (isnan (H1)) $=0 ; \quad H 2 f(i \operatorname{snan}(H 2 f))=0 ; \quad$ Cohf $(i \operatorname{snan}($ Cohf $))=0$;

$\mathrm{H} 11=\mathrm{H} 1 ; \quad \mathrm{H} 21=\mathrm{H} 2$; H11f=H1f; $\mathrm{H} 21 \mathrm{f}=\mathrm{H} 2 \mathrm{f} ; \quad \circ$ Since the above is

repeated for all tests, $\mathrm{H} 1 \mathrm{H} 2$ and their filtered counterparts are

saved as new variables

$j=[5,7,8,9,15,16,17,18] ;$ A row matrix is defined to use as plotting tool. The elements correspond to the measurements that are not present in this test

counter $=1$; $\frac{\circ}{0}$ Counter defined to be used as loop clock

\% The following part plots the H1, H2, Coherence and their filtered

o counterparts

for $i=1: 18$

if $i \sim=j$ (counter)

figure; subplot $(3,1,1)$;

plot $\left(F,(H 1(:, i)), r^{\prime}, F f,(H 1 f(:, i)), b^{\prime}\right)$; ylabel ('H1'); xlim( [0

20]); set (gca,'EontSize', 16)

suptitle(['Sensor at ', num2str(i),' Impact at 3'])

legend ('Unfiltered', 'Filtered')

subplot $(3,1,2)$;

plot $\left(\mathrm{F}, \operatorname{mag} 2 \mathrm{db}(\mathrm{H} 2(:, i)), ' r ', F f, \operatorname{mag} 2 \mathrm{db}(\mathrm{H} 2 \mathrm{f}(\mathrm{:}, \mathrm{i})), \mathrm{b}^{\prime}\right)$; legend ('Unfiltered

', 'Filtered'); ylabel ('H2'); xlim([0 20]); set (gca, 'FontSize', 16) subplot $(3,1,3)$;

plot (F, mag2db (Coh (:,i)), 'r', Ff, mag2db (Cohf (:,i)), 'b') ; legend ('Unfilter ed', 'Filtered'); xlabel ('Freq(Hz)'); ylabel ('Coherence (dB)'); xlim([0

20]) ; set (gca,' 'FontSize', 16)

else

if counter $==$ length $(j)$

else

counter=counter+1;

end

end

end

o The following part plots only the filtered Hl and Coherence for 
Filtered data eval (strcat ('TF', num2str(i), '=filtfilt (ld,T', num2str(i), ');')) eval (strcat ('IF', num2str(i),' =filtfilt (ld, I', num2str(i), ');')) eval (strcat ('TF', num2str(i),'=filtfilt (hd, TF', num2str(i),');')) eval (strcat ('IF', num2str(i),'=filtfilt (hd, IF', num2str(i), ');'))

eval (strcat (' [Pxxf', num2str(i), ', ff', num2str(i), '] =pwelch (TF', num2str( i), ', window, [],NFFT, FS); ')); \% autospectrum of the response eval (strcat ('PXXf = PXXf+Pxxf', num2str(i), ';')) eval (strcat ('Ff = Fftff', num2str(i), ';'))

eval (strcat (' [Pfff', num2str(i), ', wf', num2str(i), '] =pwelch (IF', num2str( i), ', window, [], NFFT, FS); ')); o autospectrum of the impact eval (strcat ('PFFf = PFFf+Pfff', num2str(i),';')) eval (strcat ('Wf $=W f+w f '$, num2str(i), ';'))

eval (strcat ('[Pfxf', num2str(i), ', fxf', num2str(i), '] =cpsd (IF', num2str (i ), ', TF', num2str(i), ', window, [],NFFT,Fs); ')); 응 Cross Spectrum eval (strcat ('PFXf = PFXf+Pfxf', $\left.\operatorname{num} 2 \operatorname{str}(i),{ }^{\prime} ; '\right)$ ) eval (strcat ('FXf = FXftexf', num2str(i),';'))

eval (strcat ('[Pxff', num2str(i), ', xff', num2str(i), '] =cpsd (TF', num2str (i ) ,' , IF', num2str(i), ', window, [ ], NFFT, Fs); ')) ; Cross Spectrum eval (strcat ('PXFf = PXFf+Pxff', num2str(i), ';')) end eval (strcat ('XFf = XFf+xff', num2str(i),';'))

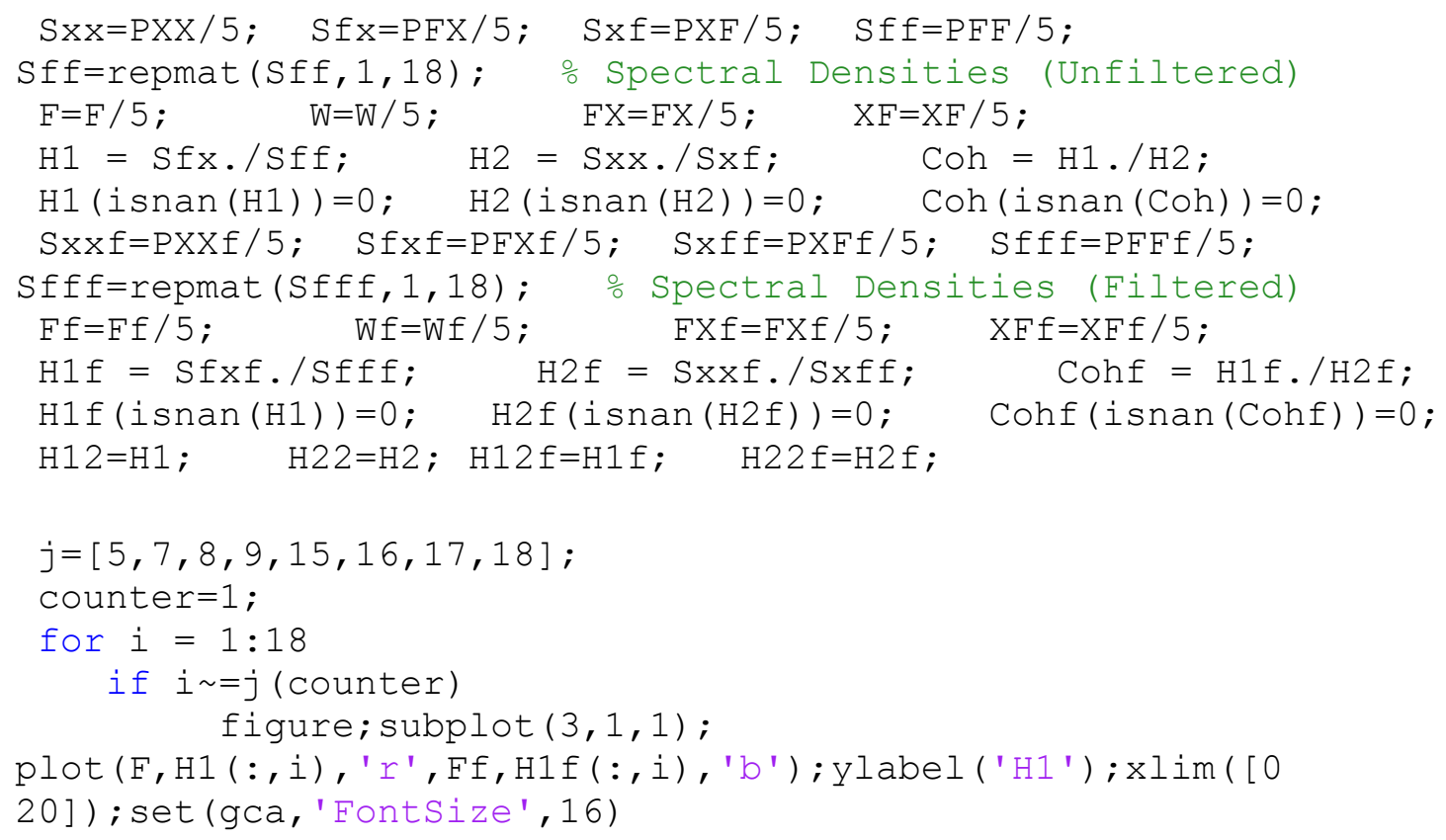




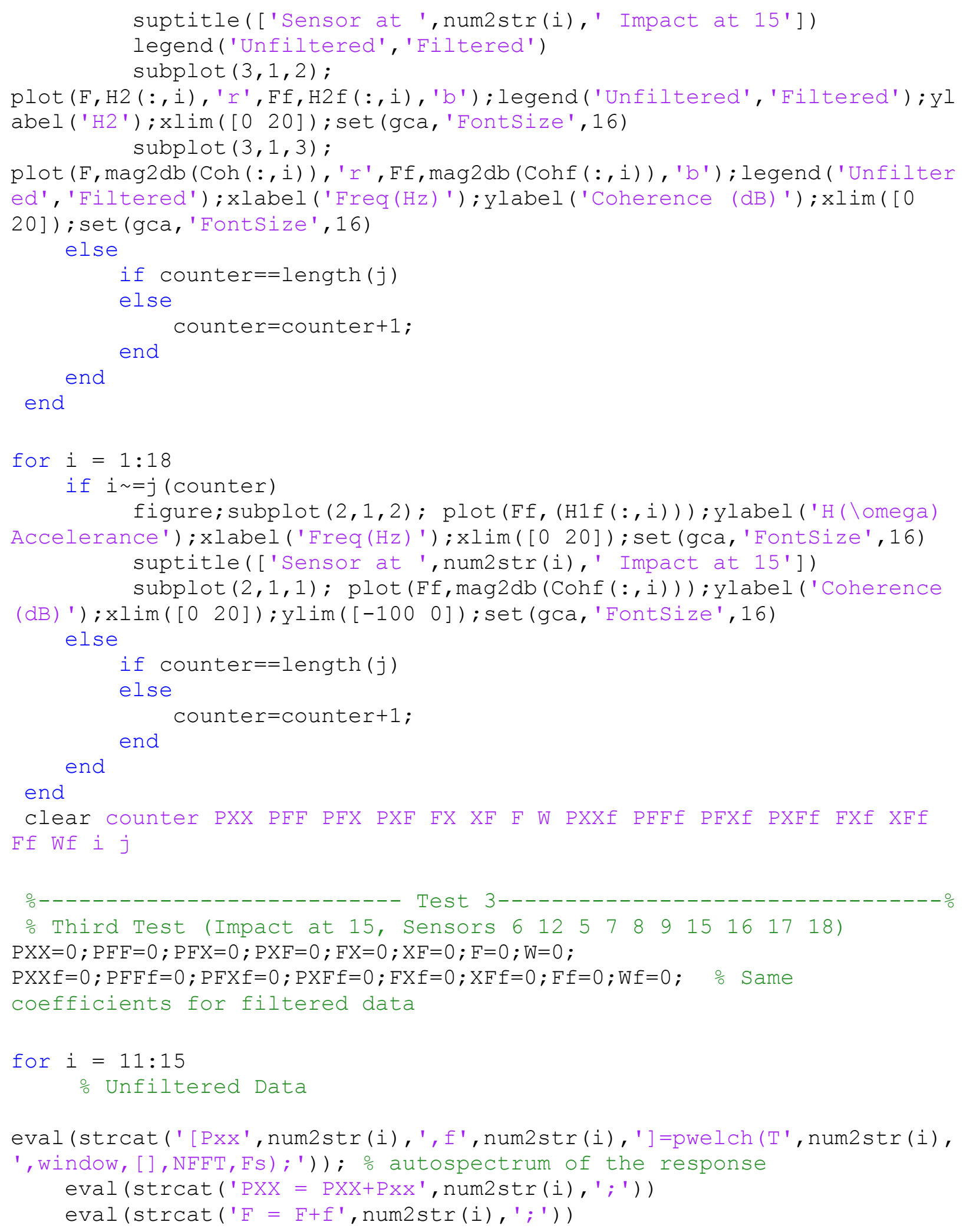




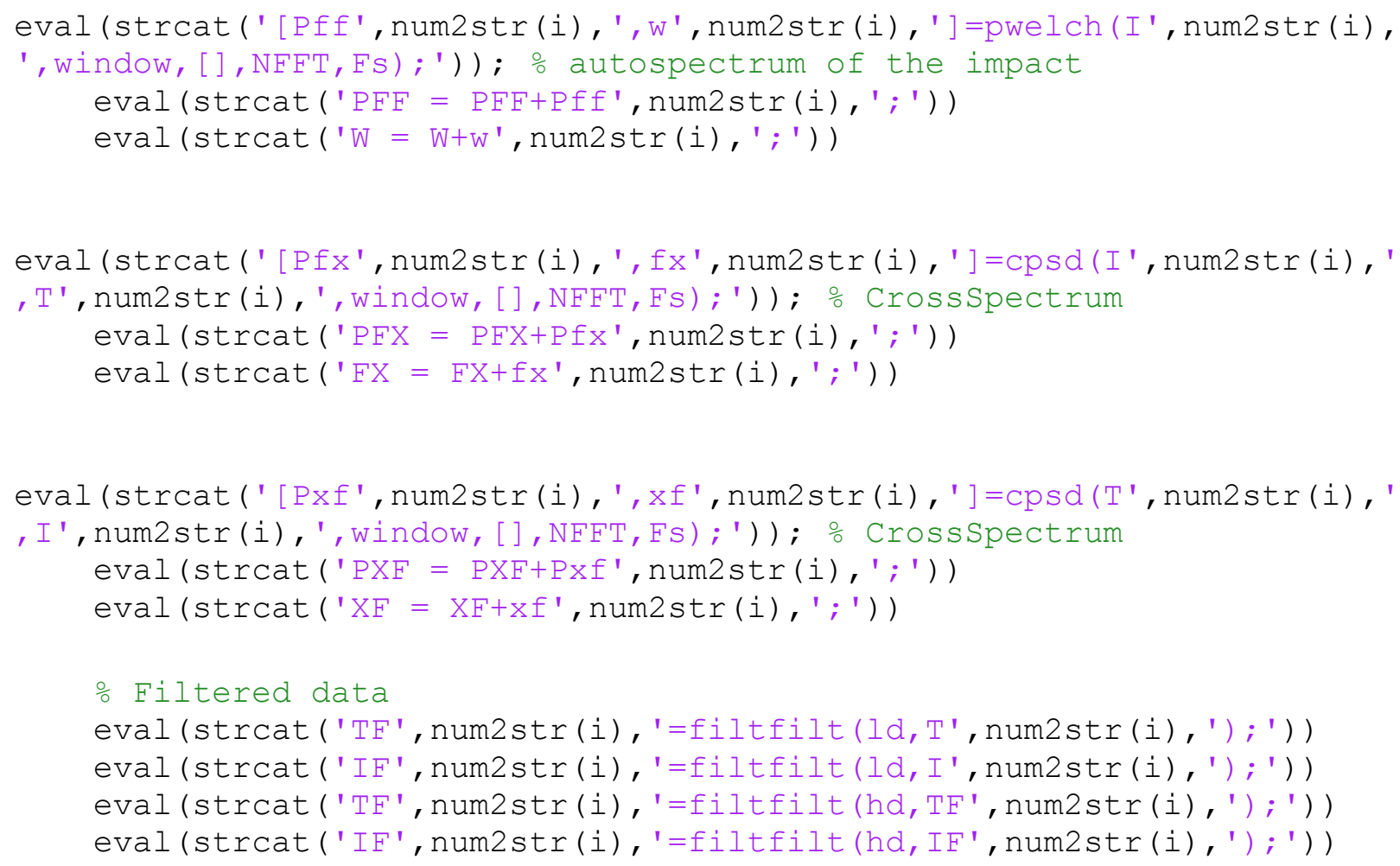

eval (strcat (' [Pxxf', num2str(i), ', ff', num2str(i), '] =pwelch (TF', num2str( i), ', window, [],NFFT, FS);')); \% autospectrum of the response eval (strcat ('PXXf = PXXf+Pxxf', num2str(i), ';')) eval (strcat ('Ff = Fffff', num2str(i), '; '))

eval (strcat (' [Pfff', num2str(i), ', wf', num2str(i), '] =pwelch (IF', num2str( i), ', window, [], NFFT, FS); ')); $\frac{\circ}{0}$ autospectrum of the impact eval (strcat ('PFFf = PFFf+Pfff', num2str(i),';')) eval (strcat ('Wf $=W f+w f '$, num2str(i), ';'))

eval (strcat ('[Pfxf', num2str(i), ', fxf', num2str(i), ']=cpsd(IF', num2str(i

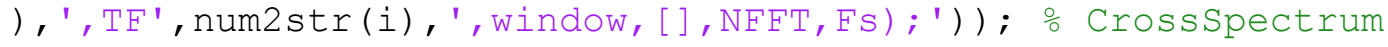
eval (strcat ('PFXf = PFXf+Pfxf', num2str $\left.(i),{ }^{\prime} ;{ }^{\prime}\right)$ ) eval (strcat ('FXf = FXftexf', num2str $\left.\left.(i),{ }^{\prime} ;{ }^{\prime}\right)\right)$

eval (strcat ('[Pxff', num2str(i), ', xff', num2str(i), '] =cpsd(TF', num2str(i ), ', IF', num2str(i), ', window, [], NFFT, Fs); ')); 응 Crosspectrum eval (strcat ('PXFf = PXFf+Pxff', num2str(i), ';')) eval (strcat ('XFf = XFf+xff', num2str(i),';'))

end

$\mathrm{SxX}=\mathrm{PXX} / 5 ; \mathrm{Sfx}=\mathrm{PFX} / 5 ; \quad \mathrm{Sxf}=\mathrm{PXF} / 5 ; \quad \mathrm{Sff}=\mathrm{PFF} / 5$; Sff=repmat (Sff, 1,18); $\quad$ Spectral Densities (Unfiltered) 


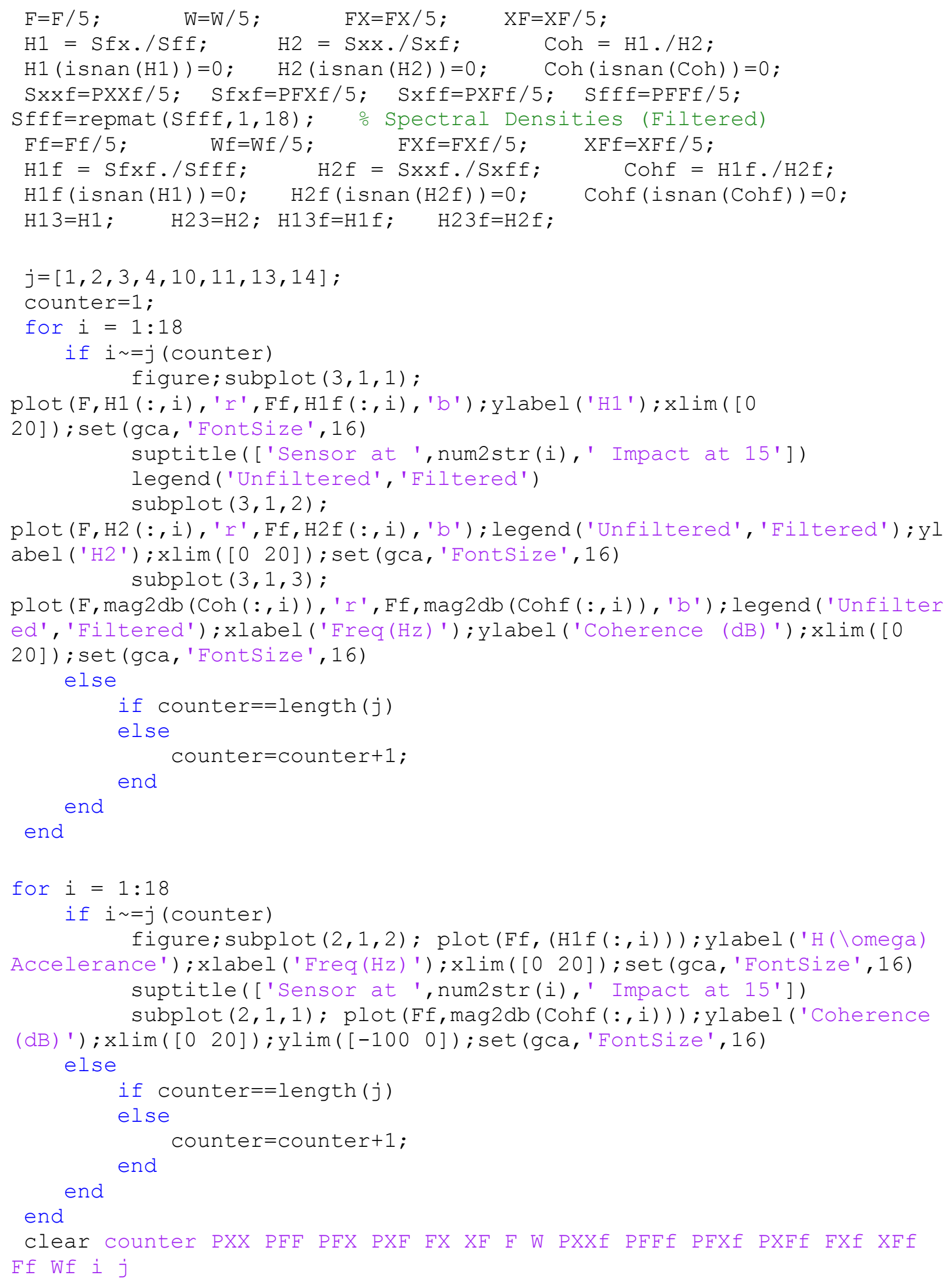




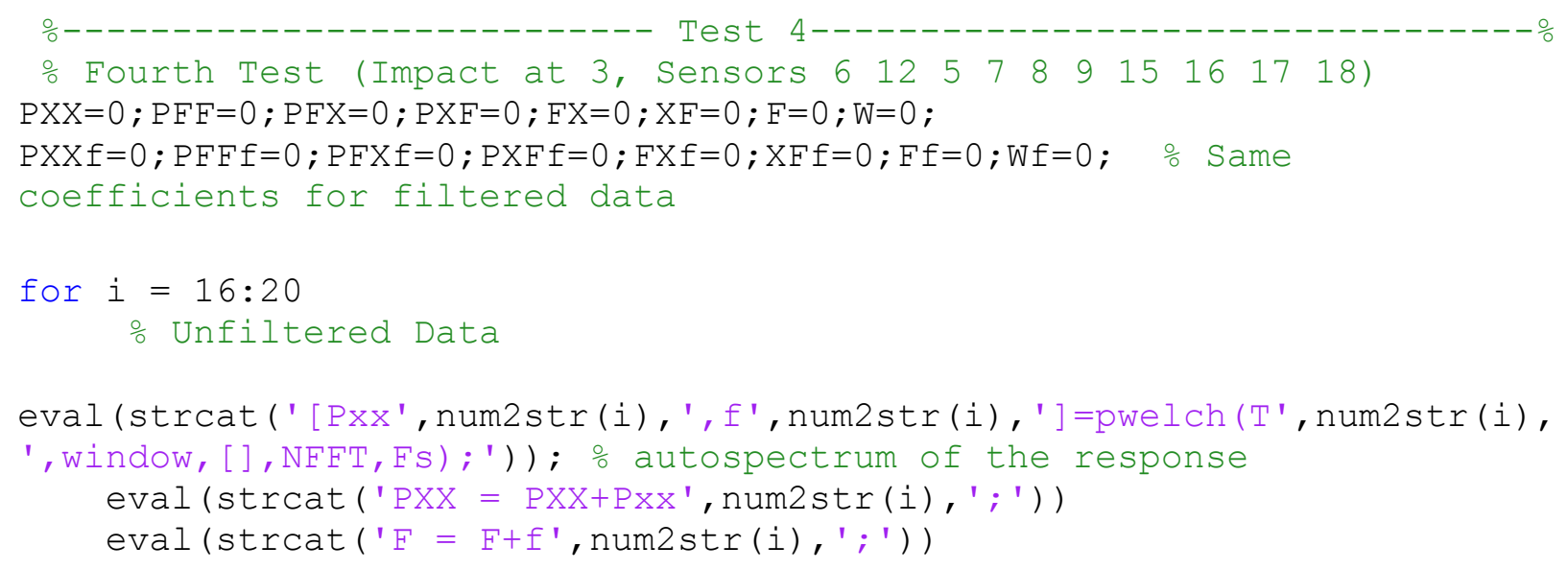

eval (strcat (' [Pff', num2str(i), ', w', num2str(i), '] =pwelch (I', num2str(i), ', window, [], NFFT, FS);')); \% autospectrum of the impact

eval (strcat ('PFF $=$ PFF+Pff', num2str(i),';'))

eval (strcat ('W $W W+W '$, num2str(i), ';'))

eval (strcat (' [Pfx', num2str(i), ', fx', num2str(i), '] =cpsd(I', num2str(i), ' ,T', num2str(i), ', window, [],NFFT, Fs); ')); \% Crossspectrum eval (strcat ('PFX = PFX+Pfx', num2str(i), ';')) eval (strcat ('FX $=F X+f X ', \operatorname{num} 2 \operatorname{str}(i), ' ; ')$ )

eval (strcat ('[Pxf', num2str(i), ', xf', num2str(i), '] =cpsd(T', num2str(i), ' , I', num2str(i), ', window, [],NFFT,Fs); ')); \% Crossspectrum eval (strcat ('PXF = PXF+Pxf', num2str(i),';')) eval (strcat ('XF $=X F+x f ', \operatorname{num} 2 \operatorname{str}(i), ' ; '))$

Filtered data eval (strcat ('TF', num2str(i), '=filtfilt (ld, T', num2str(i), '); ')) eval (strcat ('IF', num2str(i),' =filtfilt (ld, I', num2str(i),');')) eval (strcat ('TF', num2str(i),'=filtfilt (hd, TF', num2str(i), ') ;')) eval (strcat ('IF', num2str(i),'=filtfilt (hd, IF', num2str(i), ') ;'))

eval (strcat (' [Pxxf', num2str(i), ', ff', num2str(i), '] =pwelch (TF', num2str( i), ', window, [], NFFT, Fs) ; )) ; \% autospectrum of the response eval (strcat ('PXXf = PXXf+Pxxf', num2str(i), ';')) eval (strcat ('Ff = Fftff', num2str(i),';'))

eval (strcat (' [Pfff', num2str(i), ', wf', num2str(i), '] =pwelch (IF', num2str( i), ', window, [], NFFT, FS);')); $\frac{\circ}{0}$ autospectrum of the impact eval (strcat ('PFFf = PFFf+Pfff', num2str(i), ';')) eval (strcat ('Wf $=W f+w f ', \operatorname{num} 2 \operatorname{str}(i), ' ; ')$ ) 


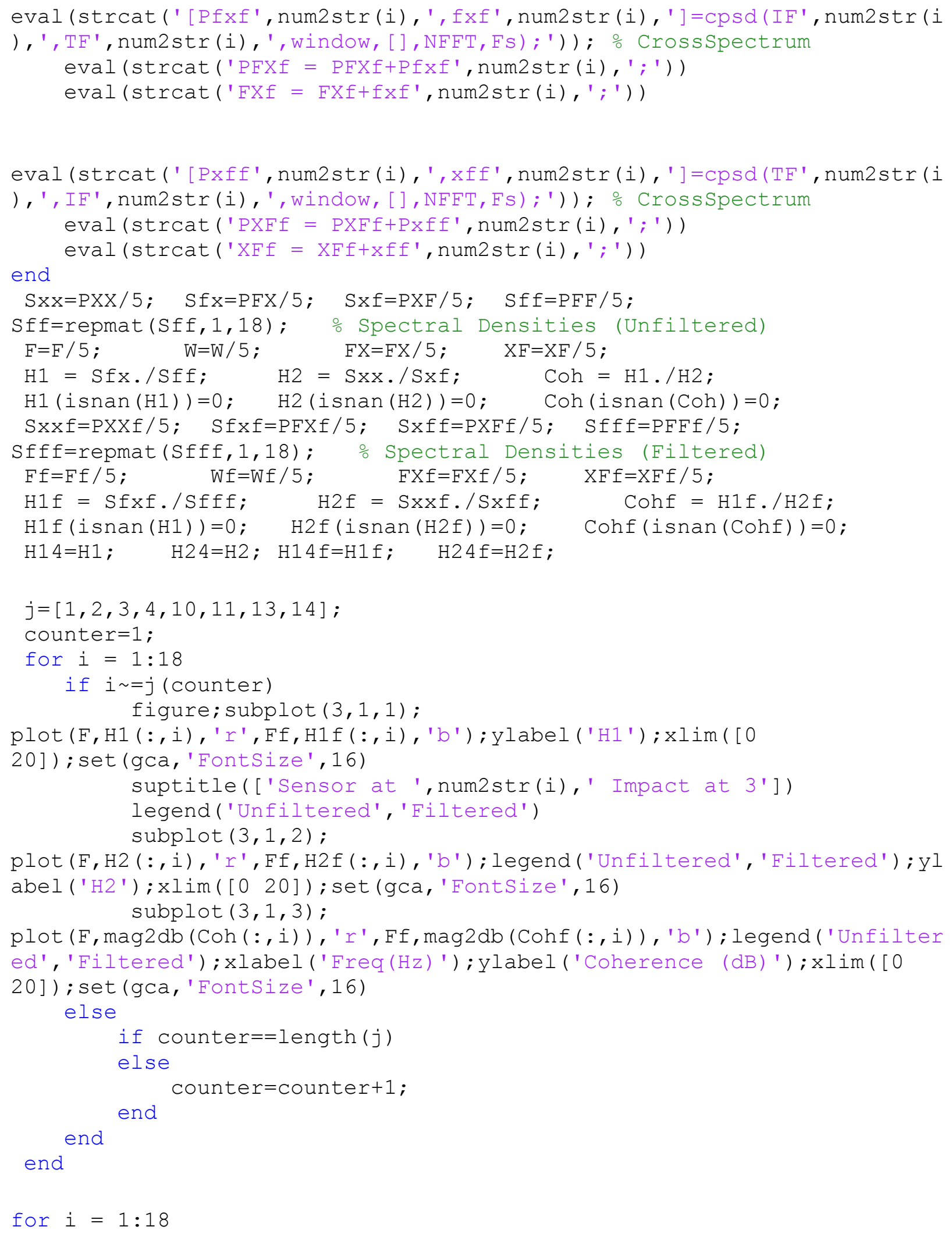


if $i \sim=j$ (counter)

figure; subplot $(2,1,2)$; plot (Ff, (H1f(:,i))) ; ylabel ('H(\omega)

Accelerance'); xlabel ('Freq(Hz) '); xlim([0 20]); set (gca, 'FontSize', 16)

suptitle(['Sensor at ', num2str(i),' Impact at 3'])

subplot $(2,1,1)$; plot (Ff, mag2db (Cohf (:,i))) ; ylabel ('Coherence

$\left.(\mathrm{dB})^{\prime}\right) ; x \lim \left(\left[\begin{array}{ll}0 & 20\end{array}\right]\right) ; y \lim ([-1000])$; set (gca,' 'FontSize', 16) else

if counter $==$ length $(j)$

else

counter=counter +1 ;

end

end

end

clear counter PXX PFF PFX PXF FX XF F W PXXf PFFf PFXf PXFf FXf XFf Ff Wf $i j$ 


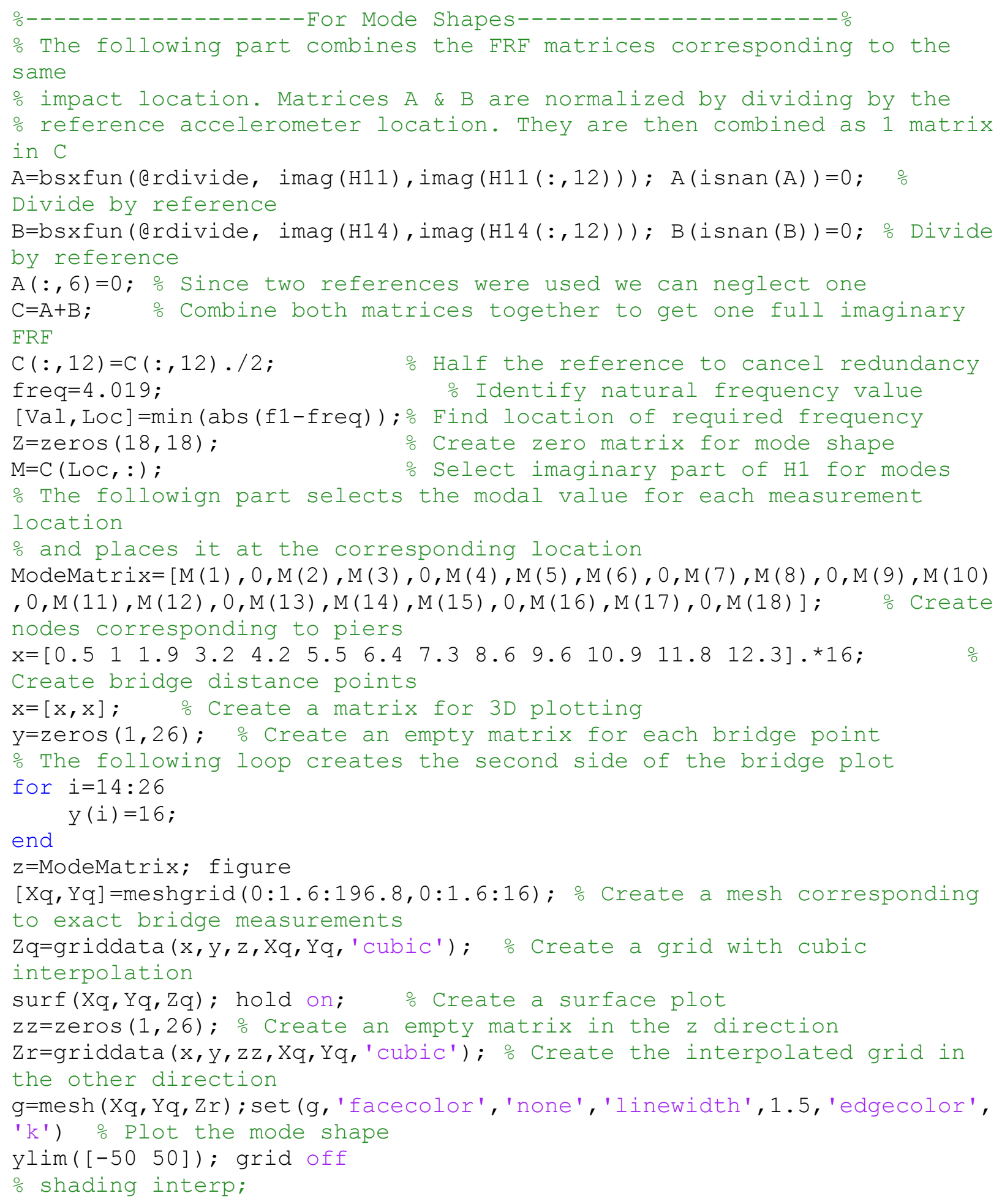




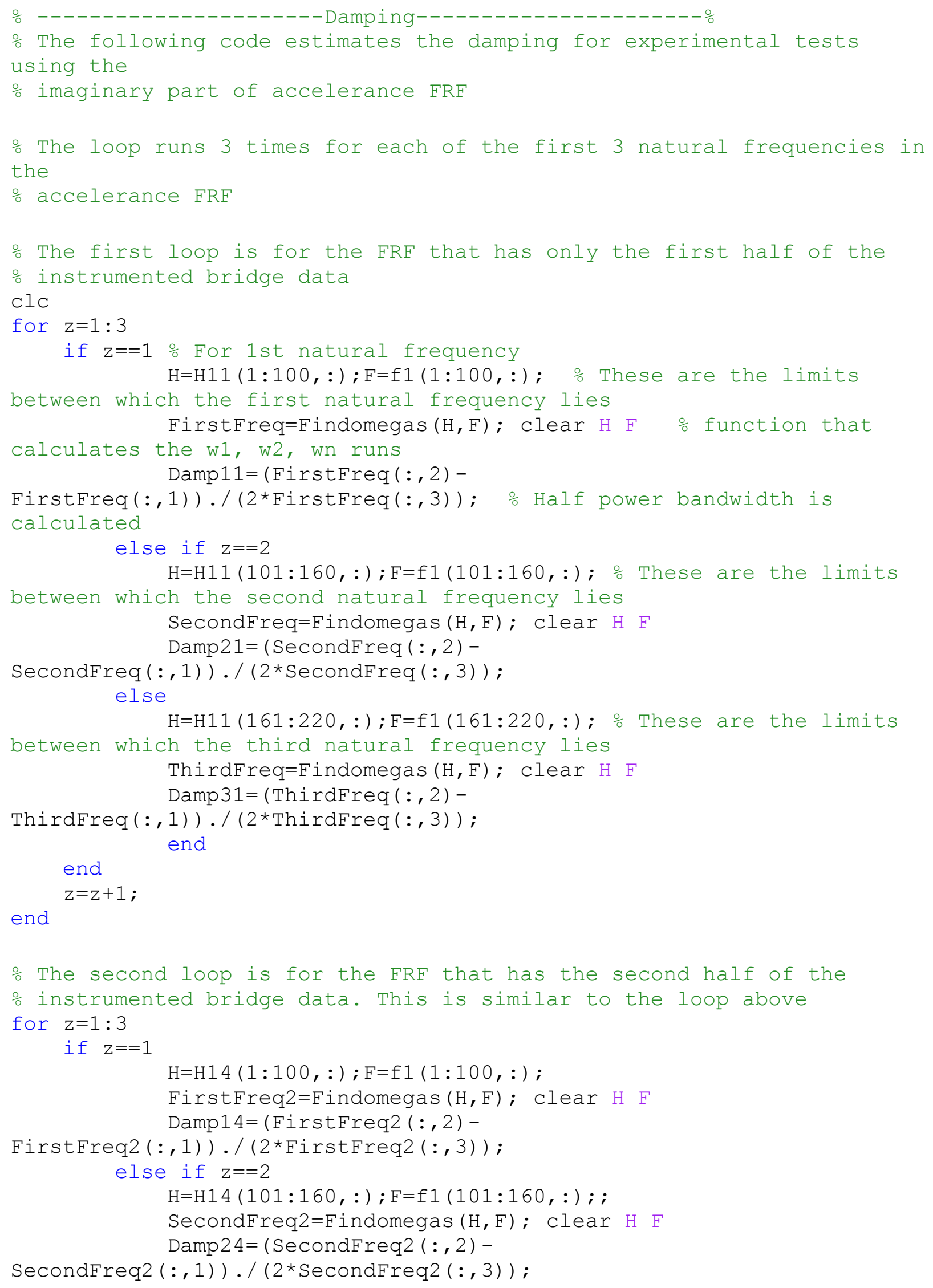




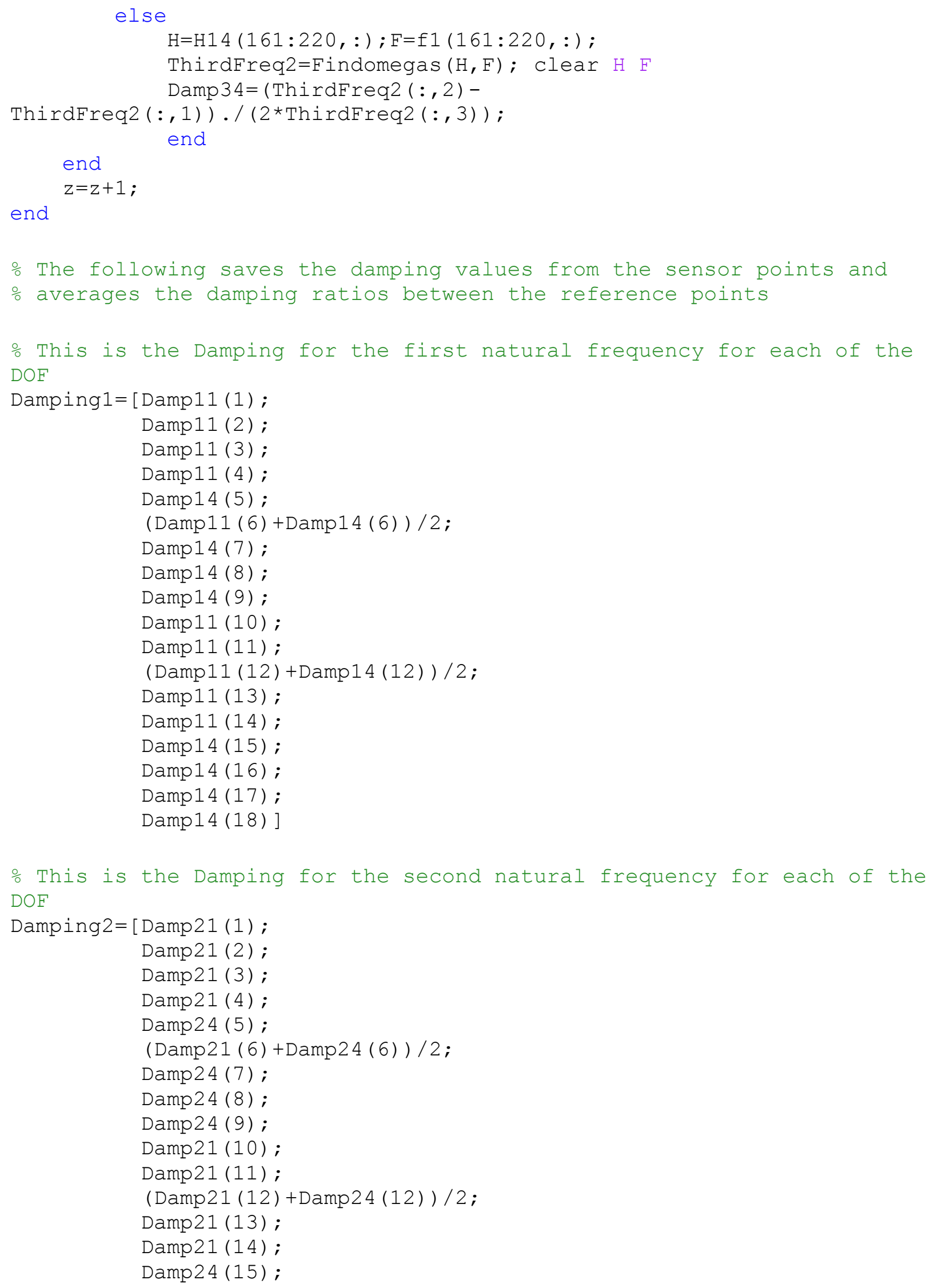




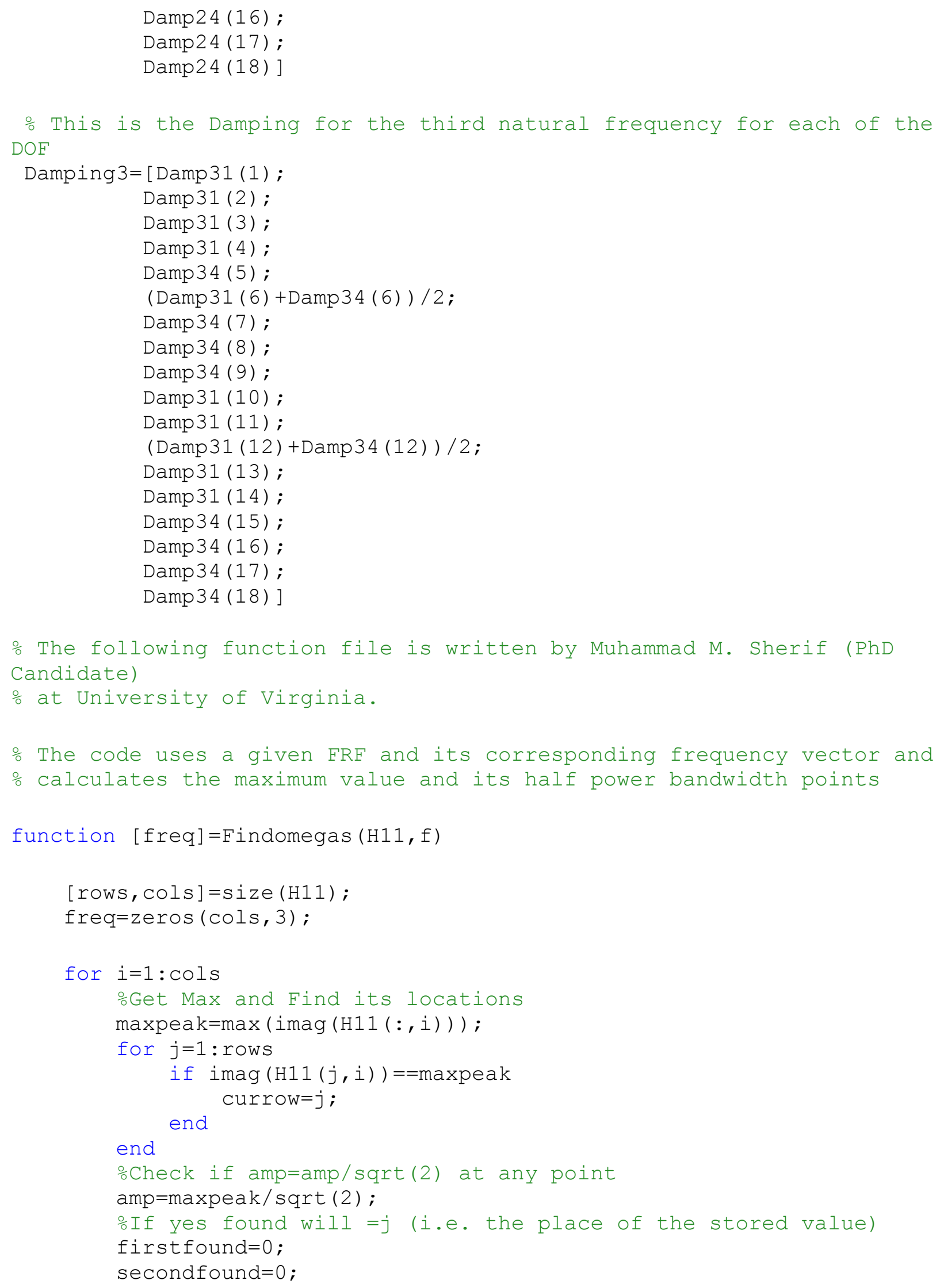




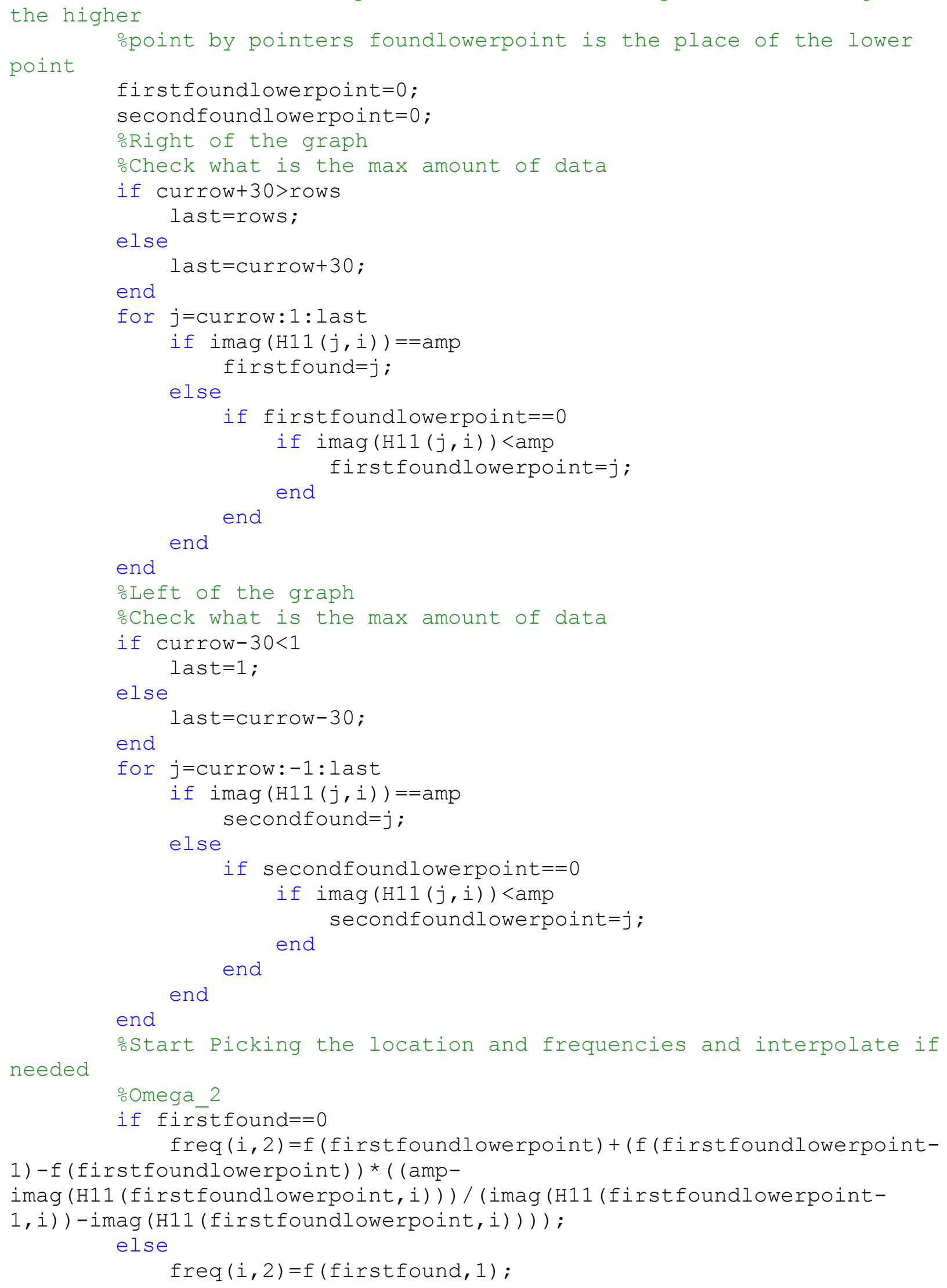




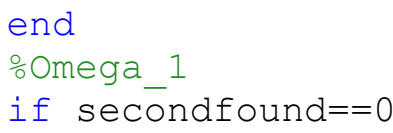

freq $(i, 1)=f($ secondfoundlowerpoint $)+(f($ secondfoundlowerpoint +1$)-$ f (secondfoundlowerpoint) )* ( (amp-

imag (H11 (secondfoundlowerpoint, i))) / (imag (H11 (secondfoundlowerpoint+1, i)) -imag (H11 (secondfoundlowerpoint,i))));

else

freq $(i, 1)=f($ secondfound, 1$)$;

end

○ Omega_n

end

freq $\left(i^{-}, 3\right)=f($ currow, 1$)$; 


\section{APPENDIX II (Operational Modal Analysis Code)}

Code for ambient vibration tests (Code is modified by the one written by Mohammad Farshchin):

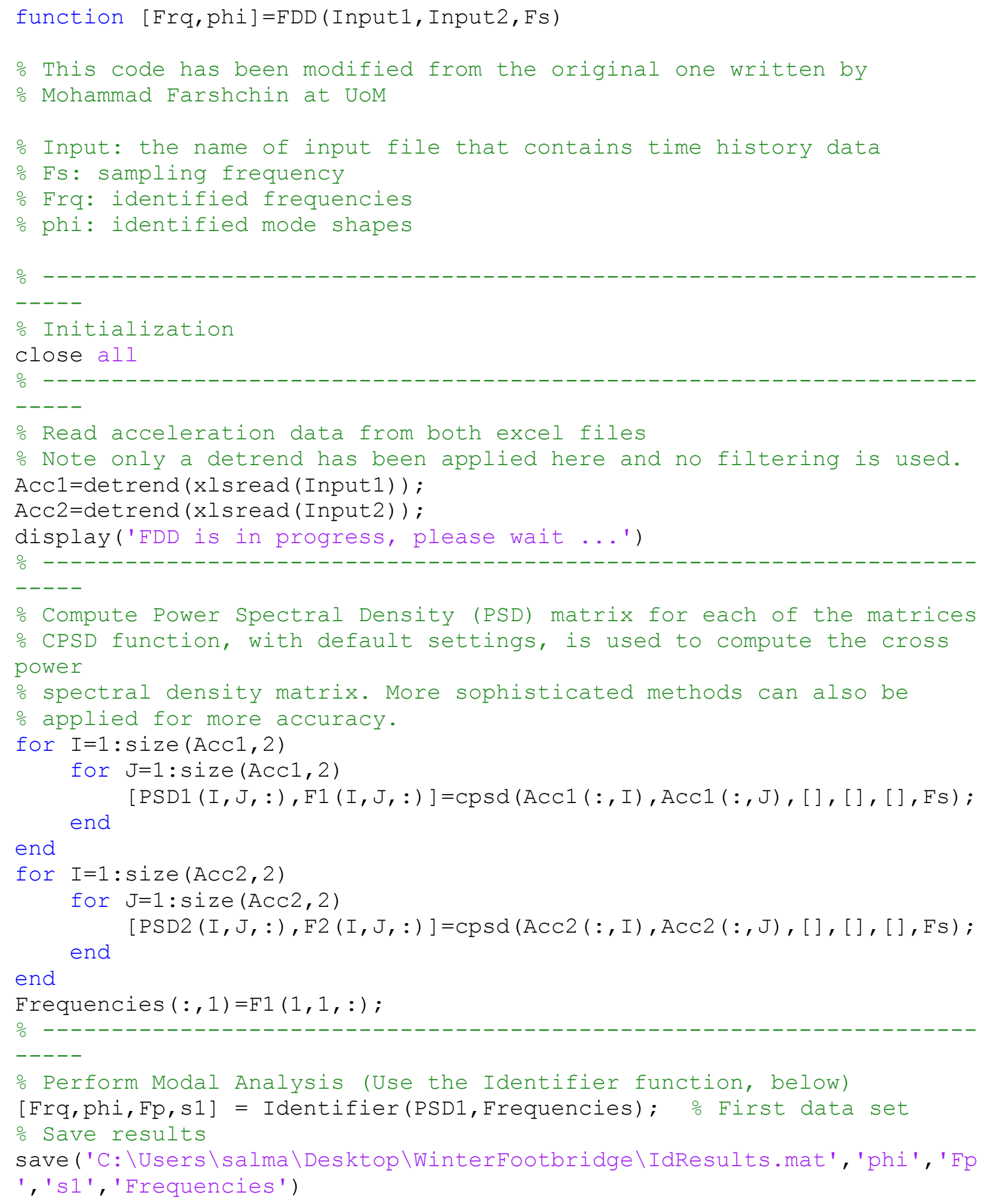


\% Repeat

[FrqX, phiX, FpX, s1X] = IdentifierX(PSD2,Frequencies); \% Second data set

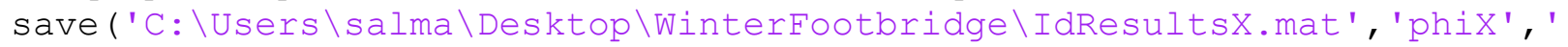
FpX', 's1X', 'Frequencies')

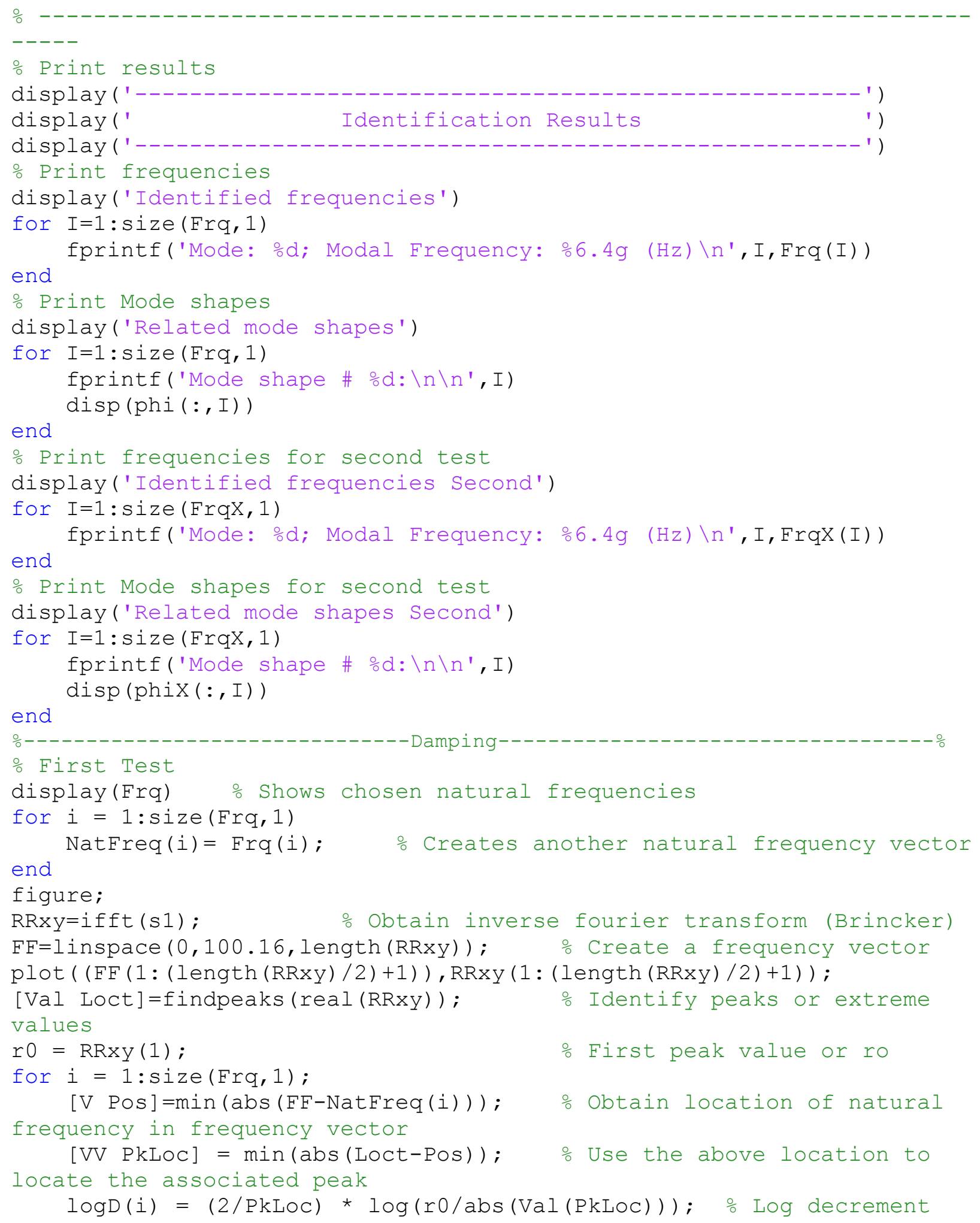




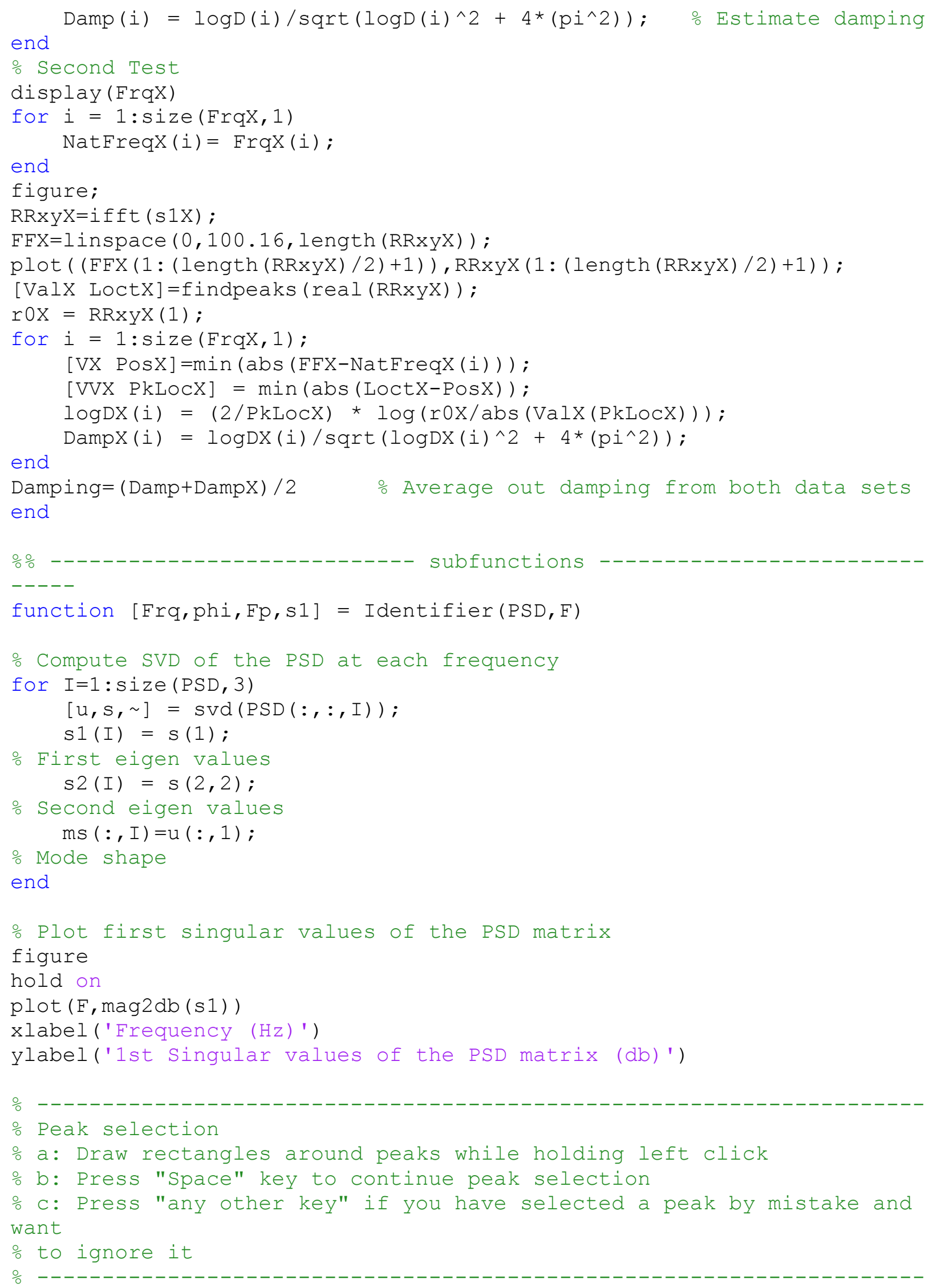


$\mathrm{Fp}=[] ; \circ$ Frequencies related to selected peaks

NumPeaks=input ('Enter the number of desired peaks:');

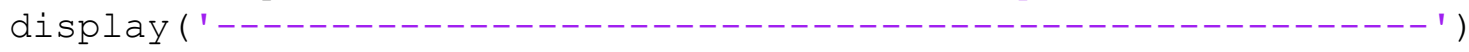

display ('Peak selection procedure')

display('a: Draw rectangles around peaks while holding left click')

display('b: Press "Space" key to continue the peak selection')

display('c: Press "any other key" if you have selected a peak by

mistake and want to ignore it')

$\mathrm{k}=0$;

while $\mathrm{k} \sim=$ NumPeaks

$\mathrm{A}=$ getrect;

\% Draw a rectangle around the peak

$[\sim, \mathrm{P} 1]=\min (\operatorname{abs}(\mathrm{F}-\mathrm{A}(1)))$;

$[\sim, \mathrm{P} 2]=\min (\operatorname{abs}(\mathrm{F}-(\mathrm{A}(1)+\mathrm{A}(3))))$;

$[\sim, \mathrm{B}]=\max (\mathrm{s} 1(\mathrm{P} 1: \mathrm{P} 2))$;

$\mathrm{Max}=\mathrm{B}+\mathrm{P} 1-1$;

\% Frequency at the selected peak

scatter (F (Max), mag2db (s1 (Max)), 'MarkerEdgeColor', 'b ' , 'MarkerFaceColor' ,'b') $\quad$ o Mark this peak

pause; key=get (gcf, 'CurrentKey') ;

$\mathrm{Fp}(\mathrm{end}+1,:)=[\operatorname{Max}, \mathrm{F}(\operatorname{Max})]$;

if strcmp (key, 'space')

o Press space to continue peak selection

$\mathrm{k}=\mathrm{k}+1$;

scatter (F (Max), mag2db (s1 (Max)), 'MarkerEdgeColor', 'g' , 'MarkerFaceColor'

,'g') \% Mark this peak as green

else

\% Press any other key to ignore this peak

Fp $(e n d,:)=[]$;

scatter(F (Max), mag2db (s1 (Max)), 'MarkerEdgeColor' , 'r' , 'MarkerFaceColor'

,'r') \% Mark this peak as red

end

end

\% Number selected peaks, respectively

$[\sim, \operatorname{Sr}]=\operatorname{sort}(\operatorname{Ep}(:, 2))$;

$\mathrm{Fp}=\mathrm{Fp}(\mathrm{Sr},:)$;

clf

$\operatorname{plot}(\mathrm{F}, \operatorname{mag} 2 \mathrm{db}(\mathrm{s} 1))$

hold on

xlabel ('Frequency (Hz)')

ylabel('1st Singular values of the PSD matrix (db)')

for $I=1: \operatorname{size}(F p, 1)$

scatter $(\operatorname{Fp}(I, 2)$, mag2db $(\mathrm{s} 1(\mathrm{Fp}(\mathrm{I}, 1)))$, 'MarkerEdgeColor' , 'g ' , 'MarkerFaceC olor', 'g')

$\operatorname{text}(\operatorname{Fp}(I, 2), \operatorname{mag} 2 \mathrm{db}(\mathrm{s} 1(\operatorname{Fp}(I, 1))) * 1.05, \operatorname{mat} 2 \operatorname{str}(I))$

end

\% Identified modal frequencies 


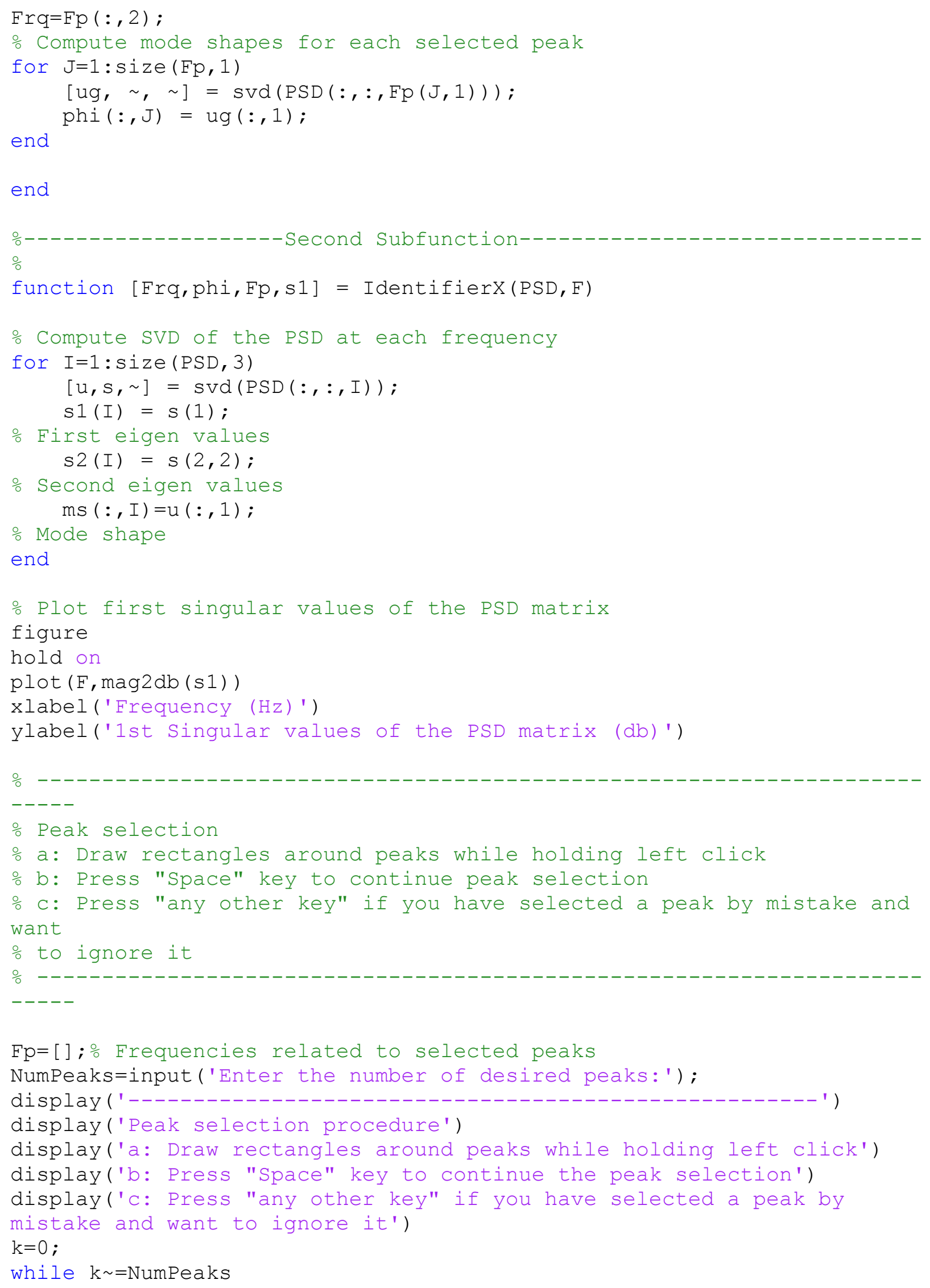


$\mathrm{A}=$ getrect;

o Draw a rectangle around the peak

$[\sim, \mathrm{P} 1]=\min (\operatorname{abs}(\mathrm{F}-\mathrm{A}(1)))$;

$[\sim, P 2]=\min (\operatorname{abs}(F-(A(1)+A(3))))$;

$[\sim, B]=\max (\mathrm{s} 1(\mathrm{P} 1: \mathrm{P} 2))$;

$\mathrm{Max}=\mathrm{B}+\mathrm{P} 1-1$;

\% Frequency at the selected peak

scatter(F (Max), mag2db (s1 (Max)), 'MarkerEdgeColor' , 'b' , 'MarkerFaceColor' ,'b') $\quad$ o Mark this peak

pause; key=get (gcf, 'CurrentKey') ;

$\mathrm{Fp}(\mathrm{end}+1,:)=[\operatorname{Max}, \mathrm{F}(\operatorname{Max})]$;

if strcmp (key, 'space')

\% Press space to continue peak selection

$\mathrm{k}=\mathrm{k}+1$;

scatter (F (Max), mag2db (s1 (Max)), 'MarkerEdgeColor' , 'g' , 'MarkerFaceColor' ,'g') \% Mark this peak as green

else

o Press any other key to ignore this peak

Fp (end, : $)=[]$;

scatter(F (Max), mag2db (s1 (Max)), 'MarkerEdgeColor' , 'r', 'MarkerFaceColor'

,'r') \% Mark this peak as red

end

end

\% Number selected peaks, respectively

$[\sim, \operatorname{Sr}]=\operatorname{sort}(\operatorname{Fp}(:, 2))$;

$\mathrm{Fp}=\mathrm{Fp}(\mathrm{Sr},:)$;

clf

$\operatorname{plot}(\mathrm{F}, \operatorname{mag} 2 \mathrm{db}(\mathrm{s} 1))$

hold on

xlabel ('Frequency ( $\mathrm{Hz})$ ')

ylabel('1st Singular values of the PSD matrix (db)')

for $I=1$ : size $(F p, 1)$

scatter $(\operatorname{Fp}(I, 2)$, mag2db (s1 (Fp (I, 1))), 'MarkerEdgeColor' , 'g ' , 'MarkerFaceC olor', 'g')

$\operatorname{text}(\operatorname{Fp}(I, 2), \operatorname{mag} 2 \mathrm{db}(\mathrm{s} 1(\mathrm{Fp}(I, 1))) \star 1.05, \operatorname{mat} 2 \mathrm{str}(I))$

end

\% Identified modal frequencies

$\operatorname{Frq}=\mathrm{Fp}(:, 2)$;

o Compute mode shapes for each selected peak

for $J=1$ : size $(\mathrm{Fp}, 1)$

$[\mathrm{ug}, \sim, \sim]=\operatorname{svd}(\operatorname{PSD}(:,:, \operatorname{Fp}(J, 1)))$;

$\operatorname{phi}(:, J)=\operatorname{ug}(:, 1)$;

end

end 


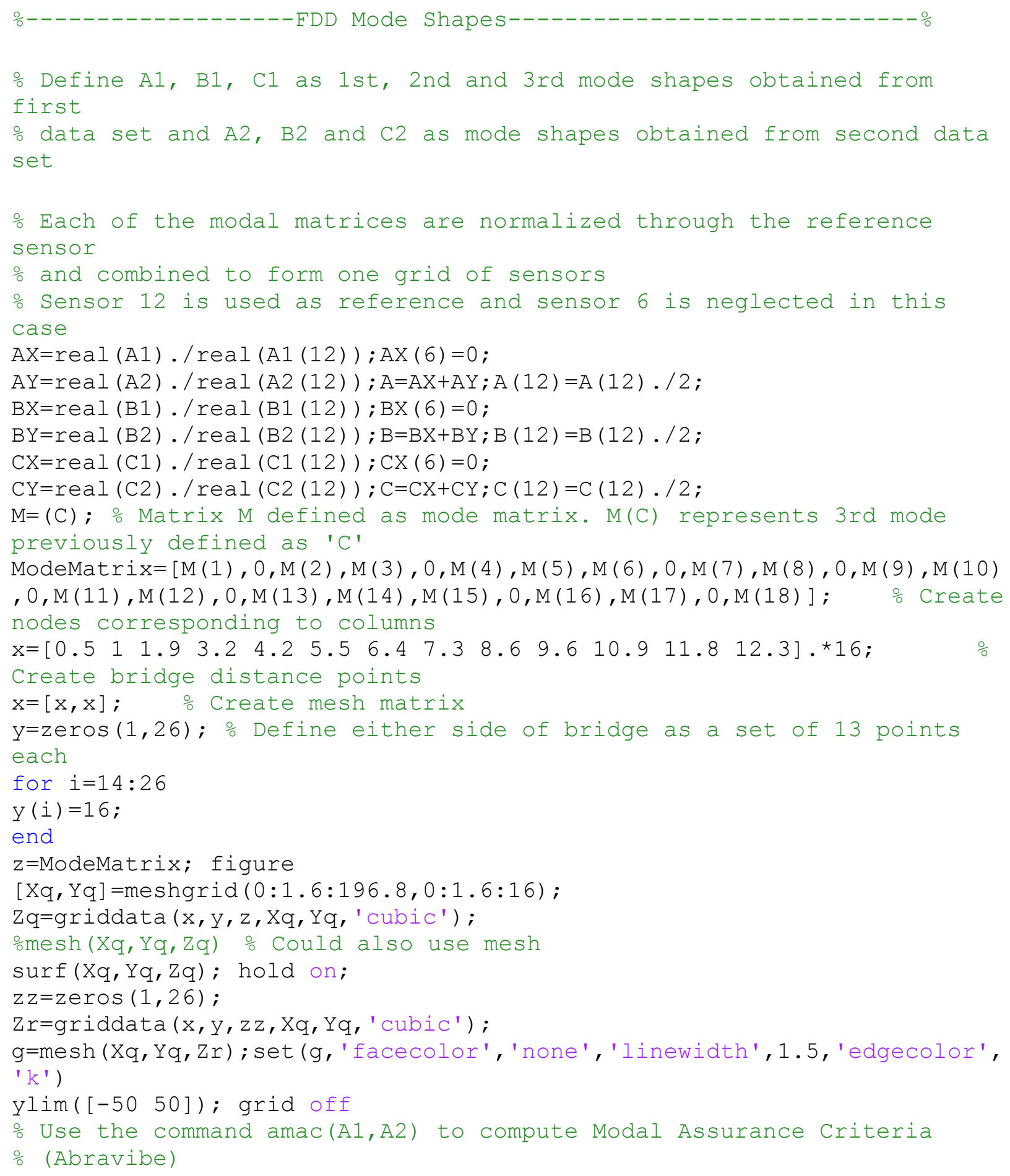




\section{APPENDIX III (Serviceability Code)}

The following code finds peak accelerations to be further used for serviceability assessment:

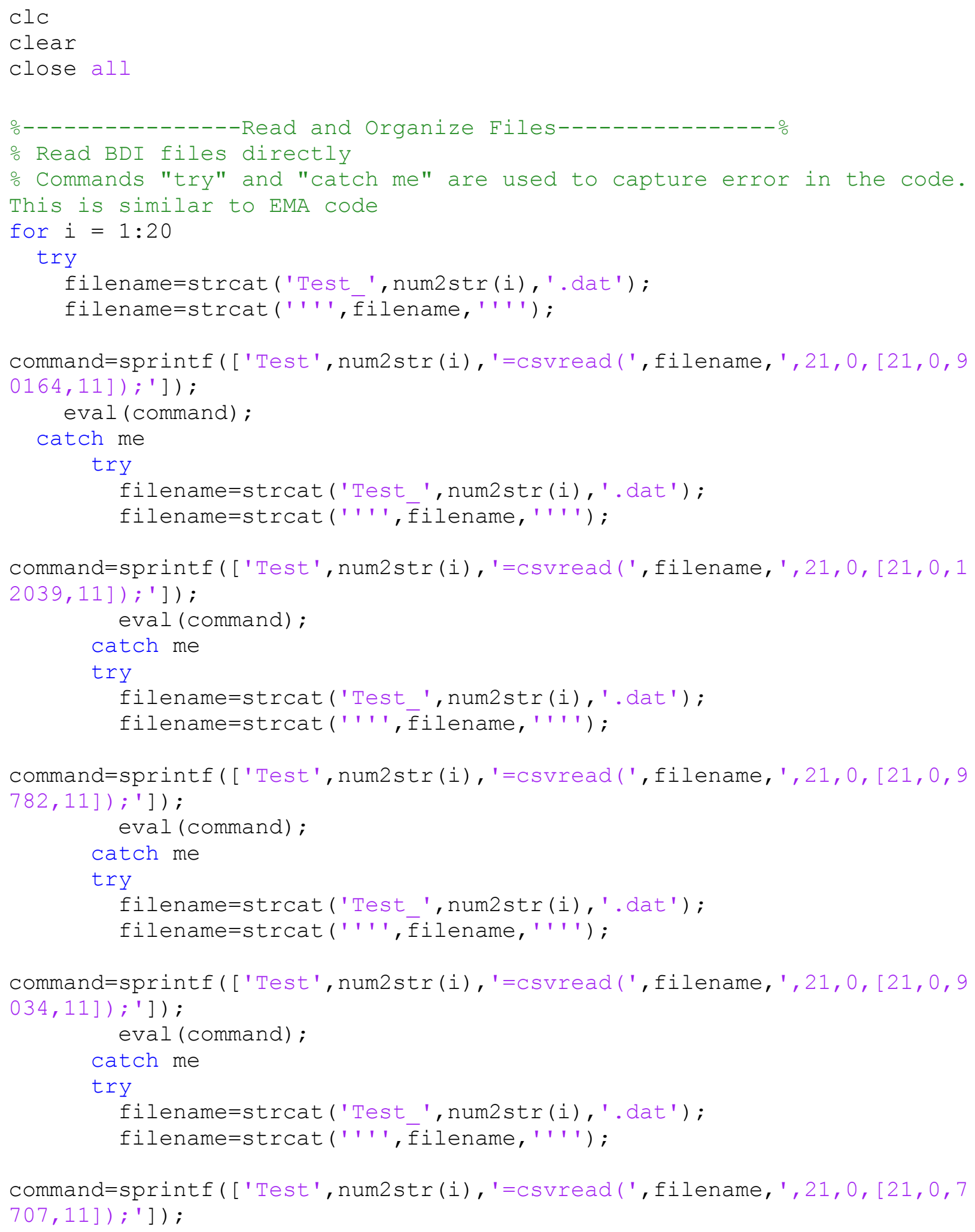




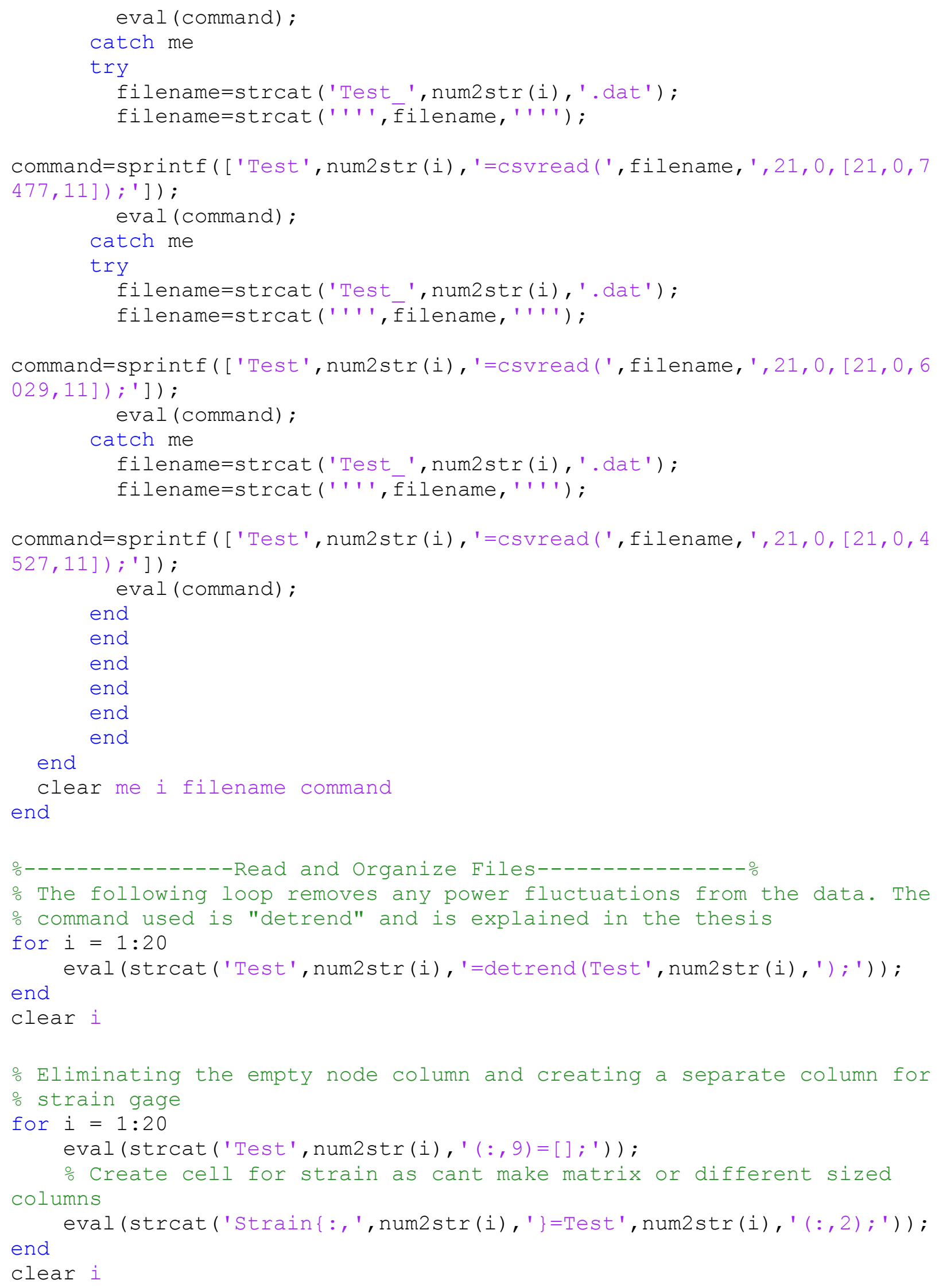




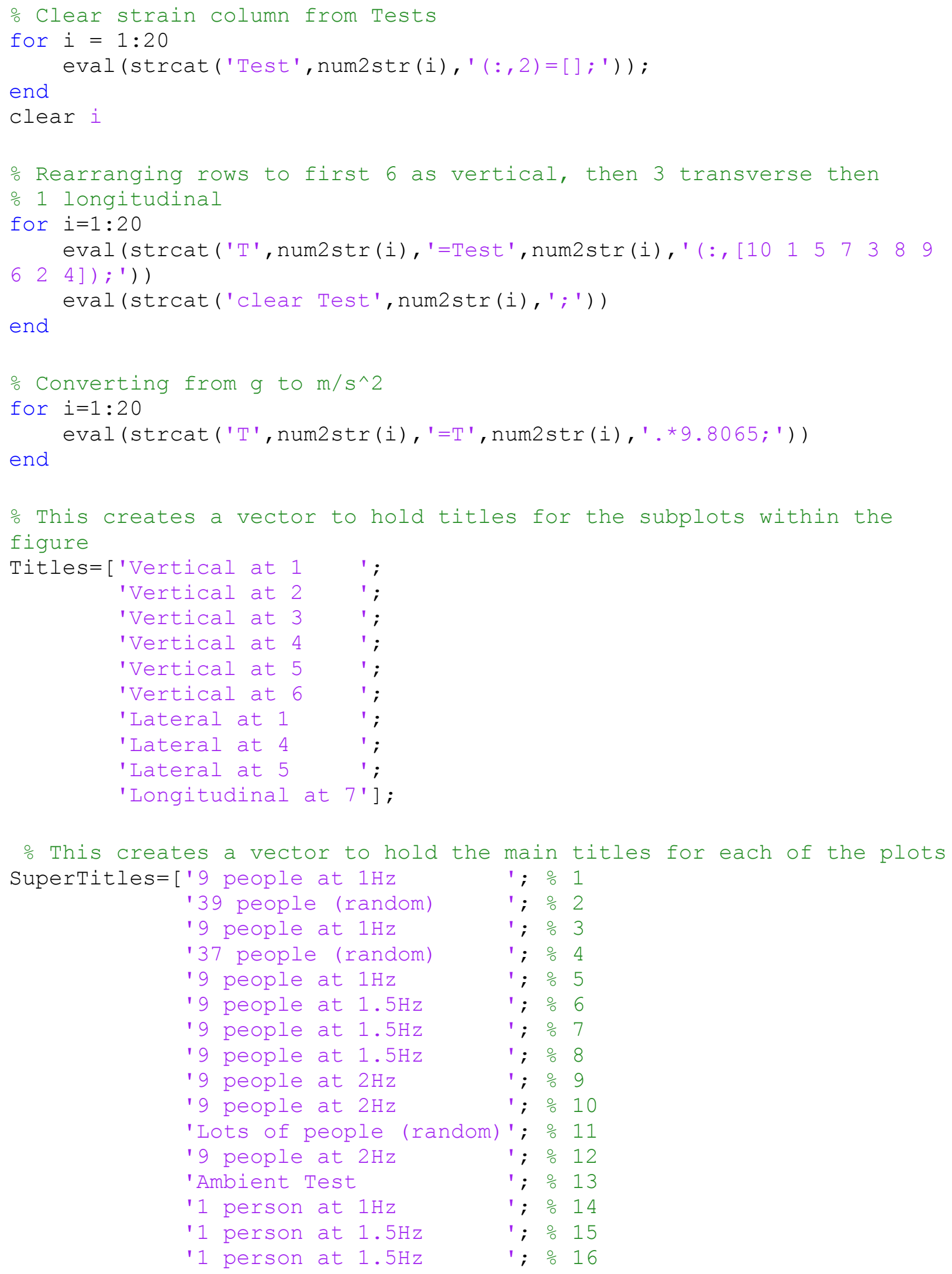


$\begin{array}{ll}\text { '1 person at } 1.5 \mathrm{~Hz} & \text { '; } \div 17 \\ \text { '1 person at } 2 \mathrm{~Hz} & \text { '; } \div 18 \\ \text { '1 person at } 2 \mathrm{~Hz} & \text { '; \% } 19 \\ \text { '1 person at } 2 \mathrm{~Hz} & \text { '] ; } \% 20\end{array}$

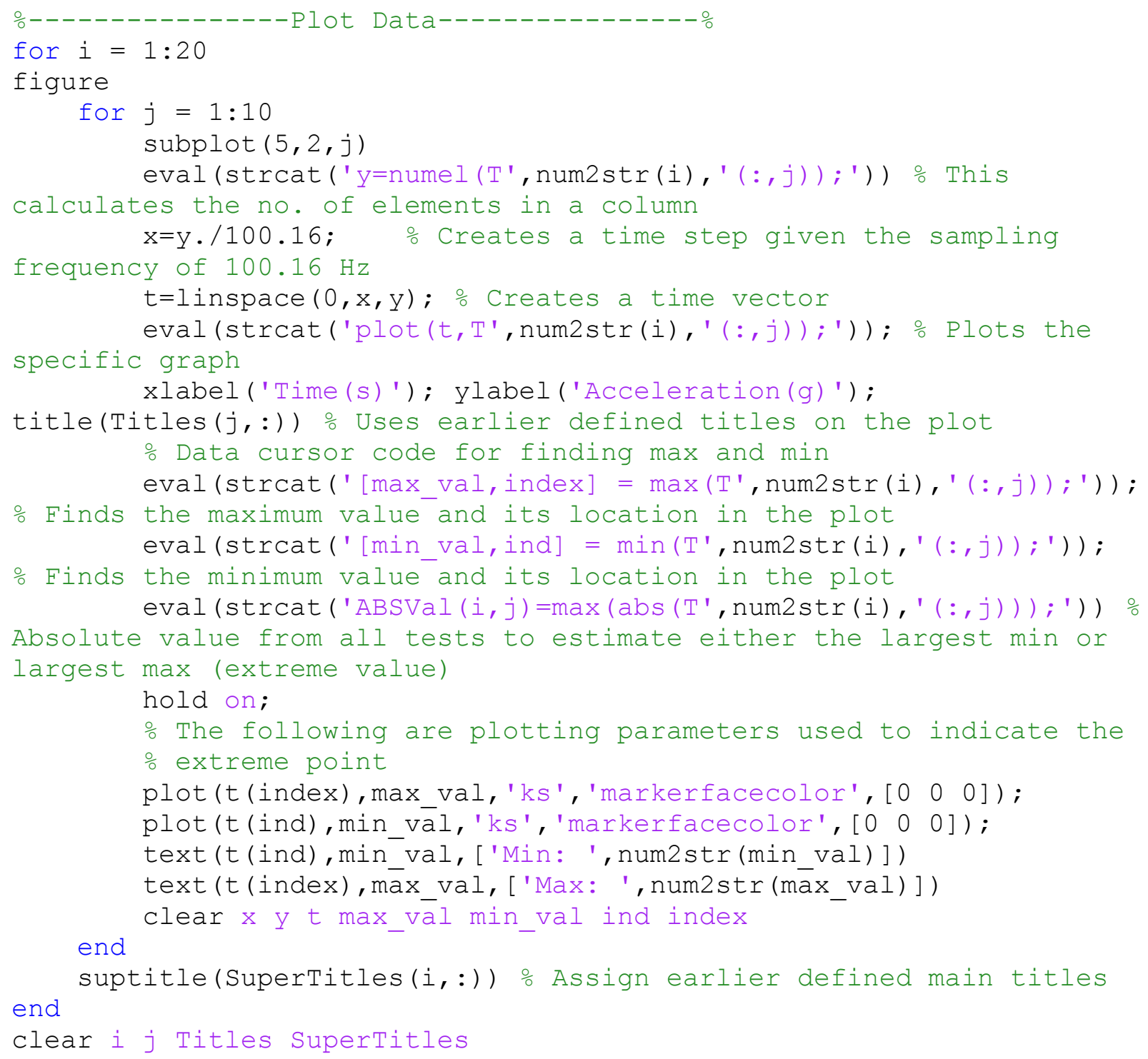




\section{APPENDIX IV (FRF Plots from EMA)}

The following are the plots from impact hammer test (Table 14) where each of the peaks corresponding to a particular degree of freedom and the impact location are shown:
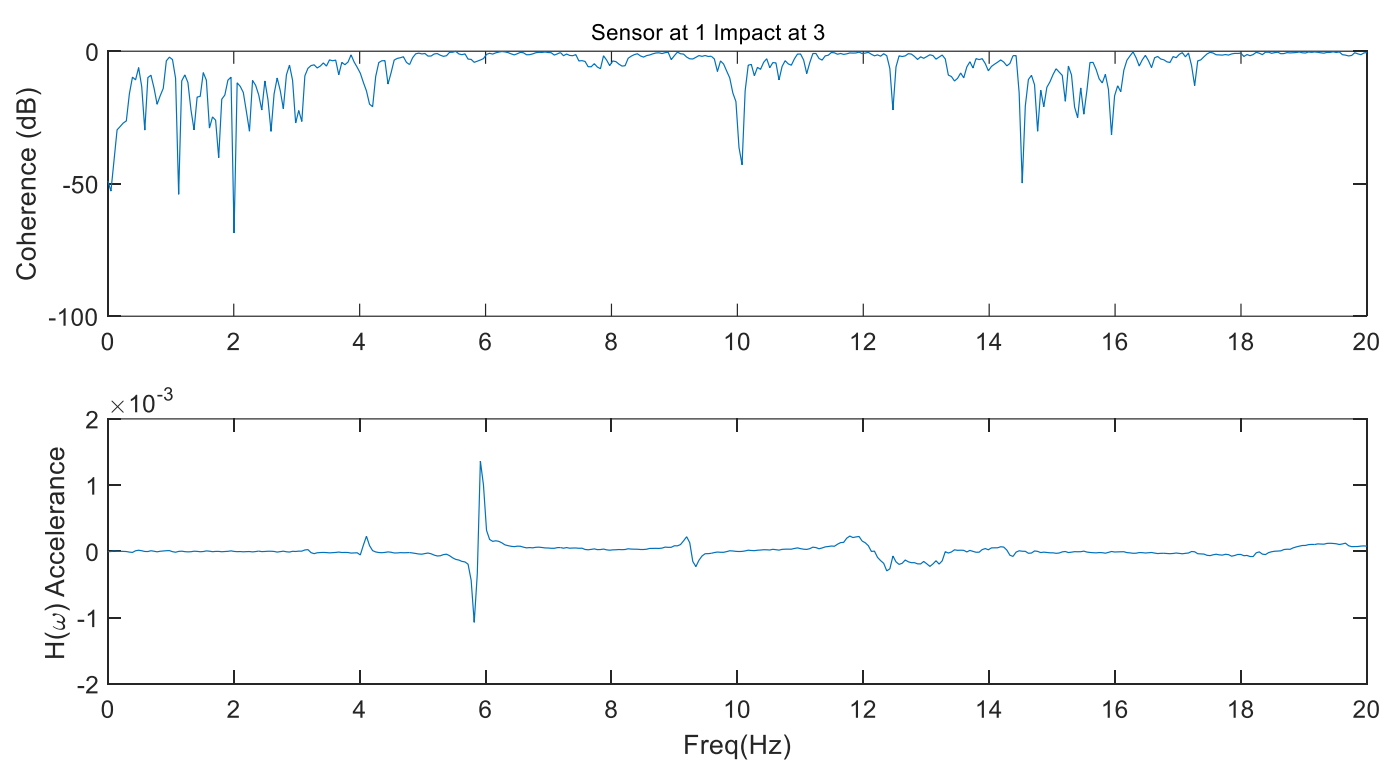

Figure 28. FRF from impact at location 3 and measurement at location 1
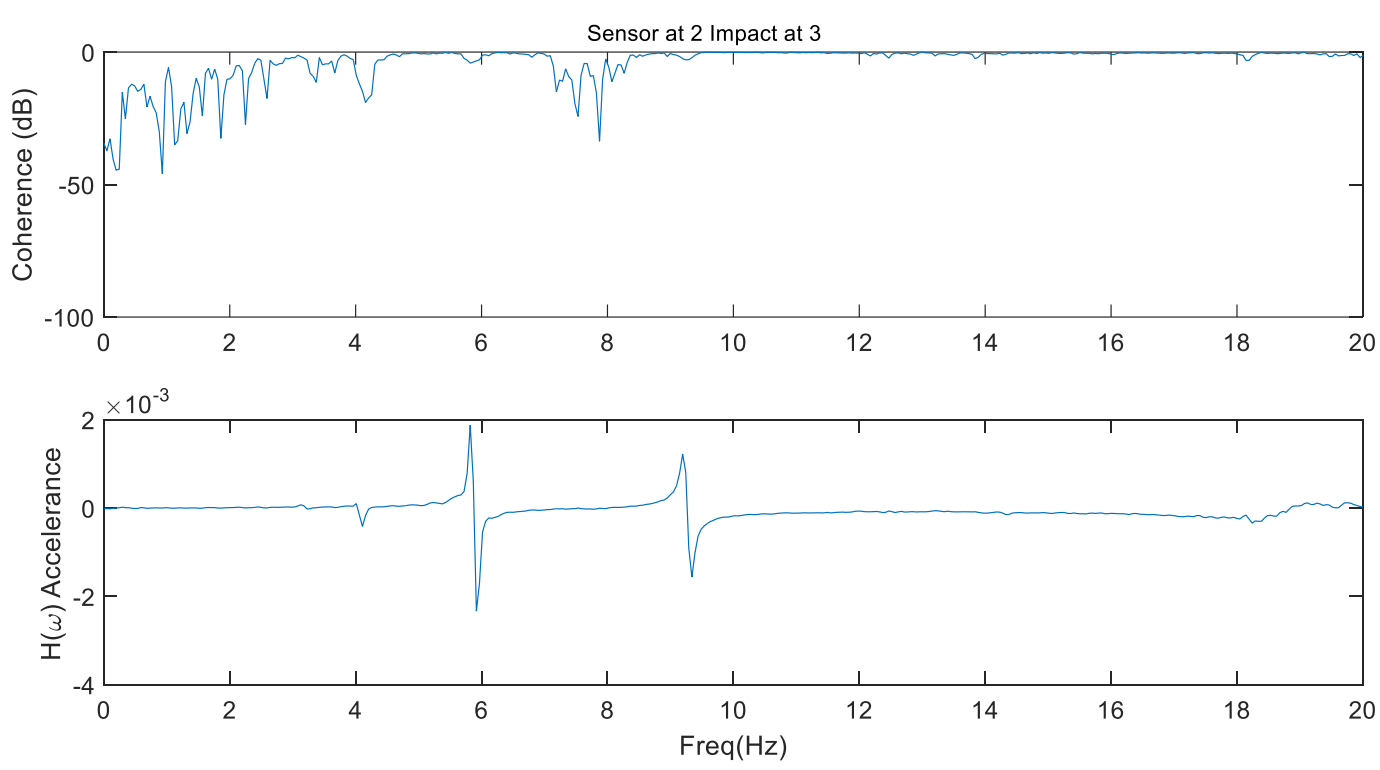

Figure 29. FRF from impact at location 3 and measurement at location 2 

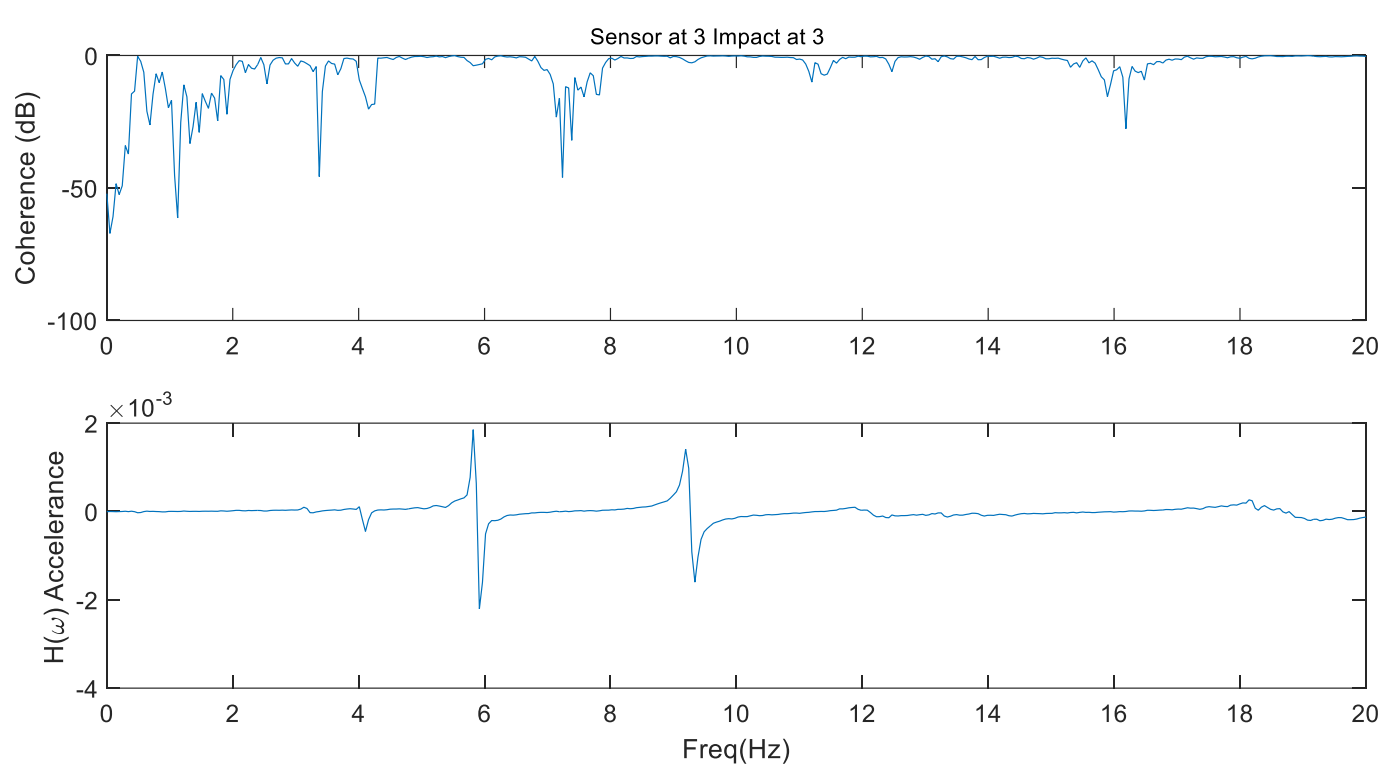

Figure 30. FRF from impact at location 3 and measurement at location 3
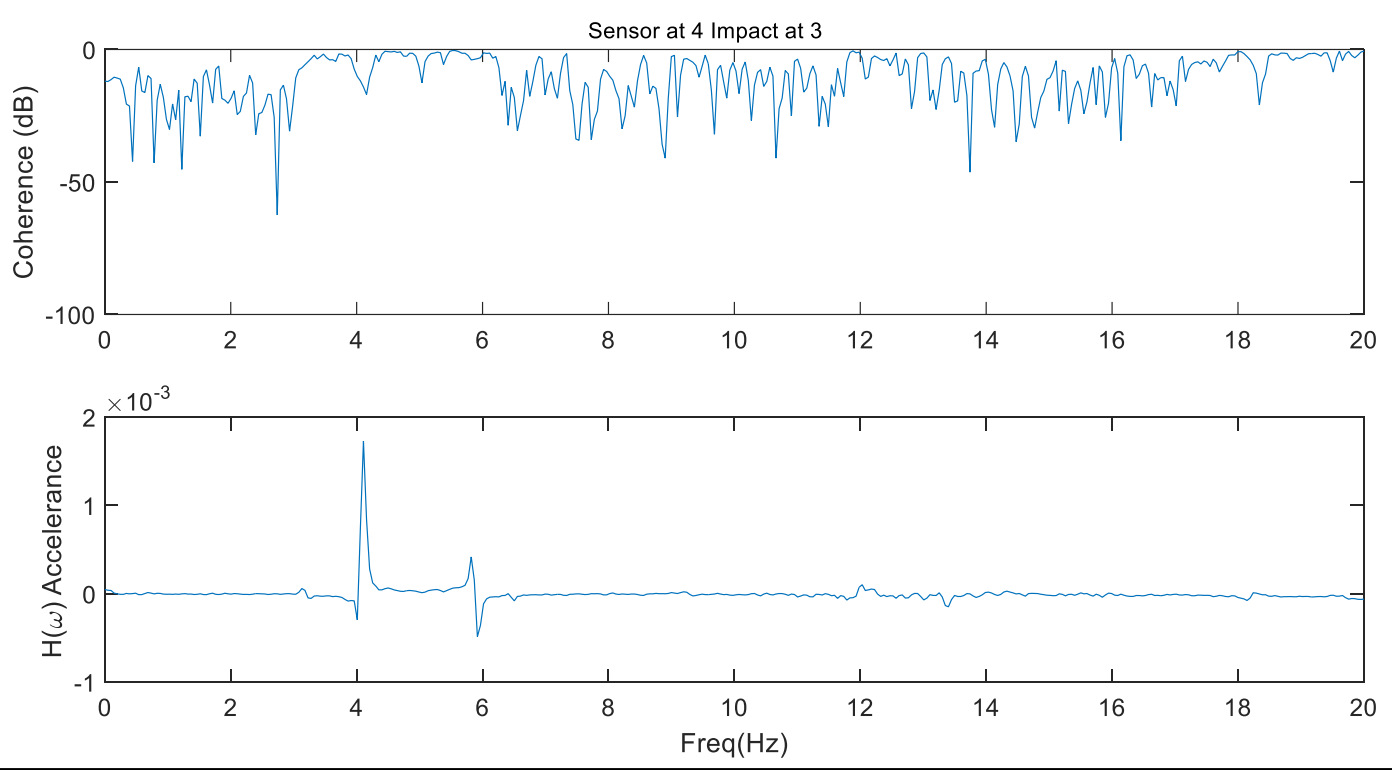

Figure 31. FRF from impact at location 3 and measurement at location 4 

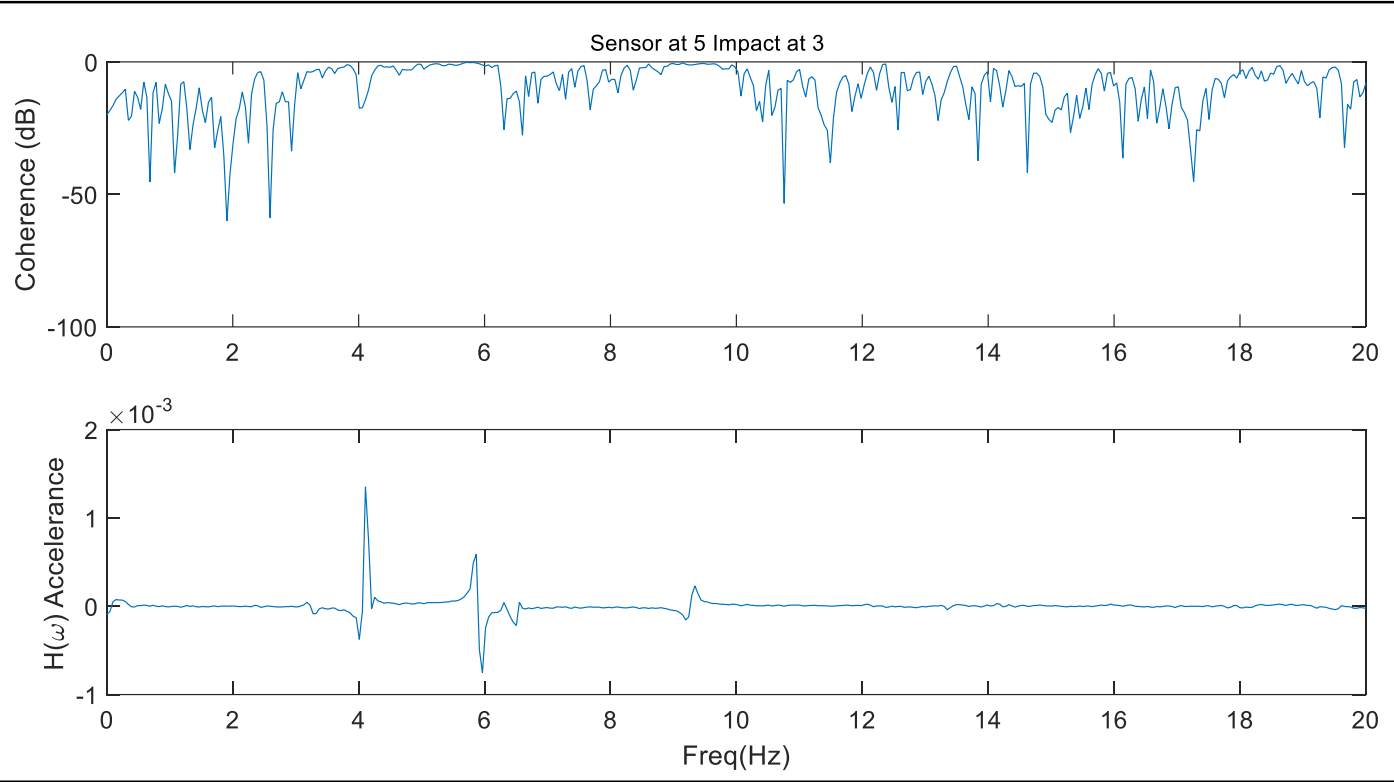

Figure 32. FRF from impact at location 3 and measurement at location 5
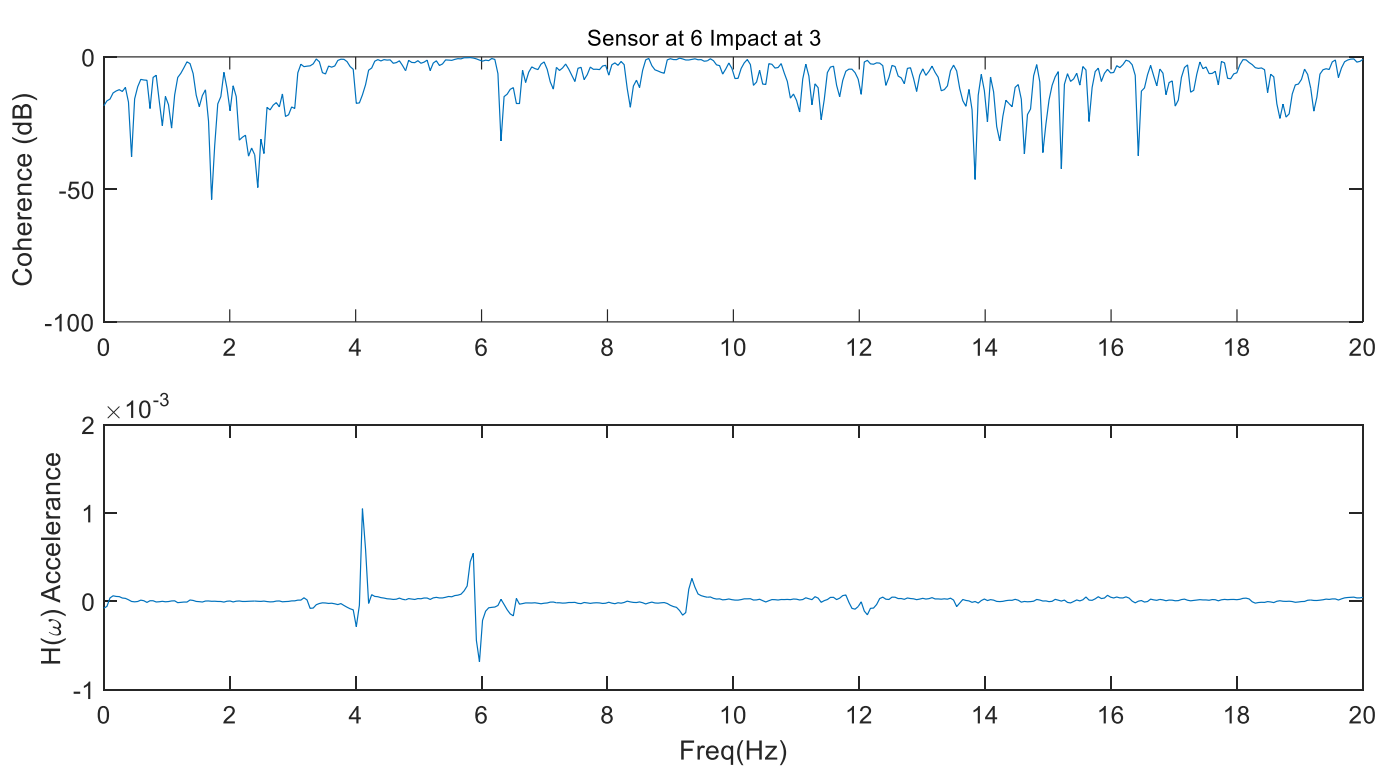

Figure 33. FRF from impact at location 3 and measurement at location 6 

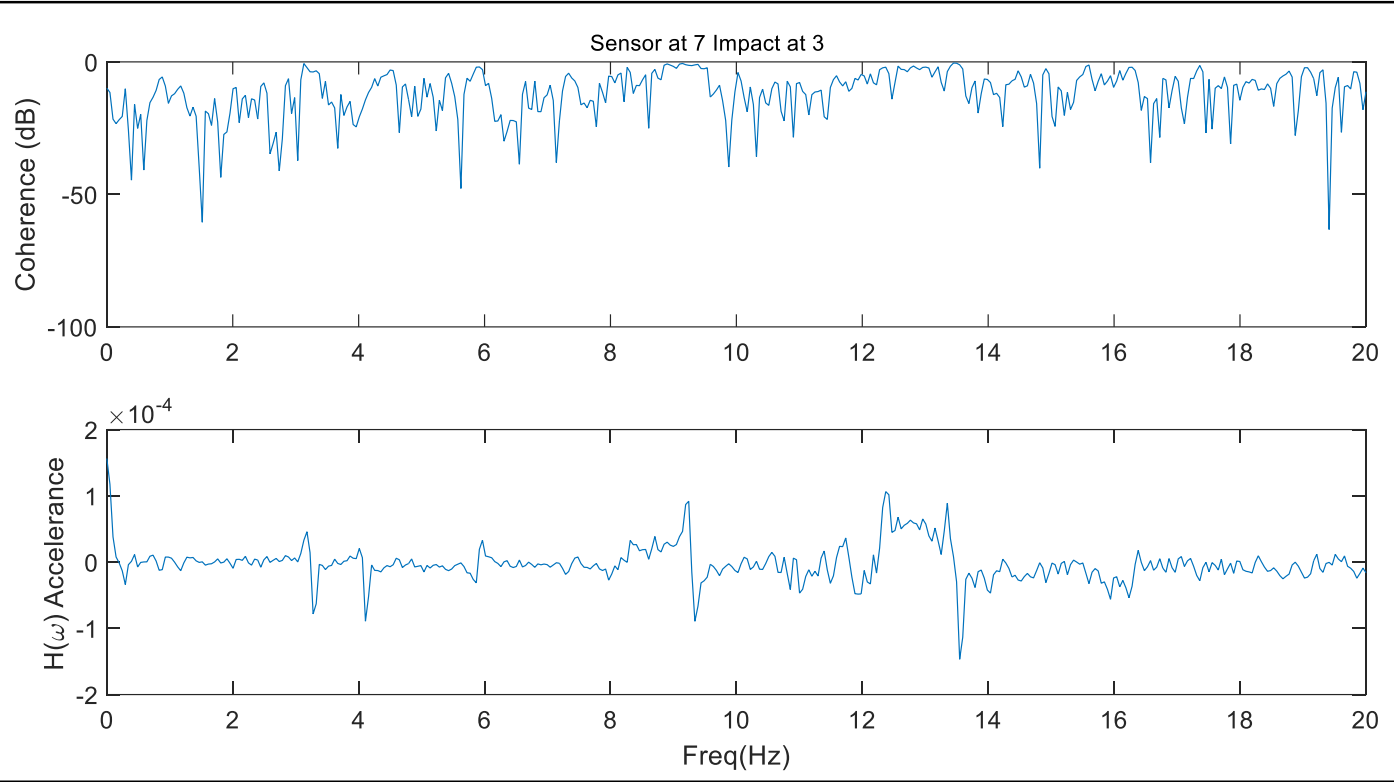

Figure 34. FRF from impact at location 3 and measurement at location 7
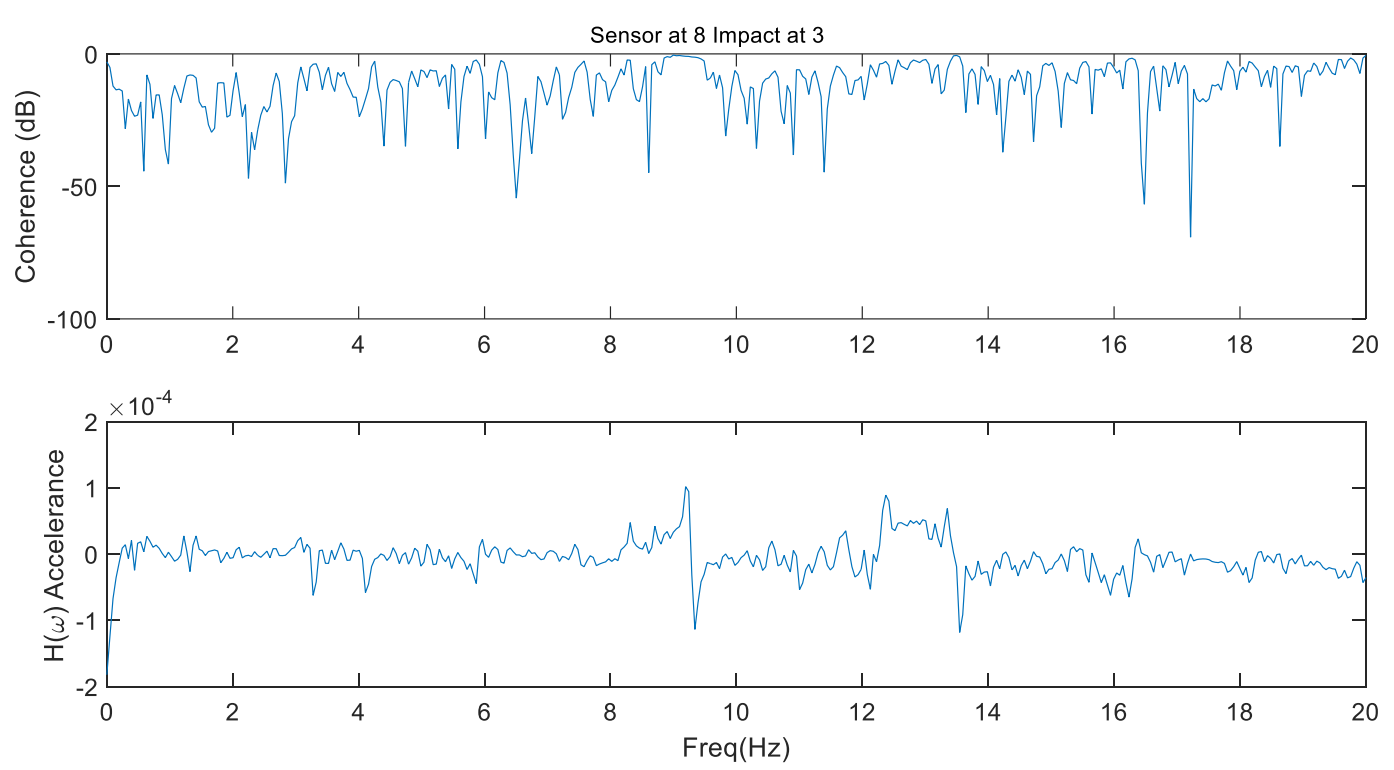

Figure 35. FRF from impact at location 3 and measurement at location 8 

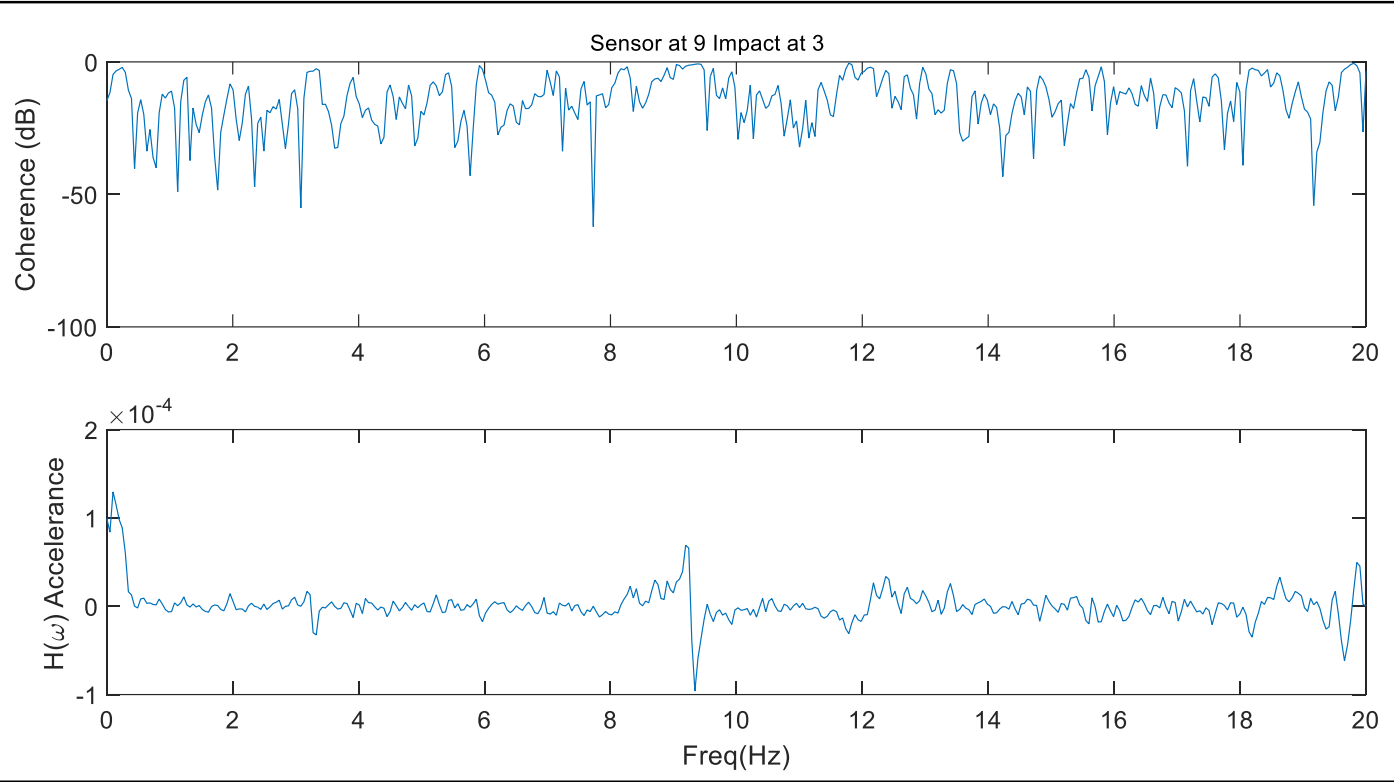

Figure 36. FRF from impact at location 3 and measurement at location 9
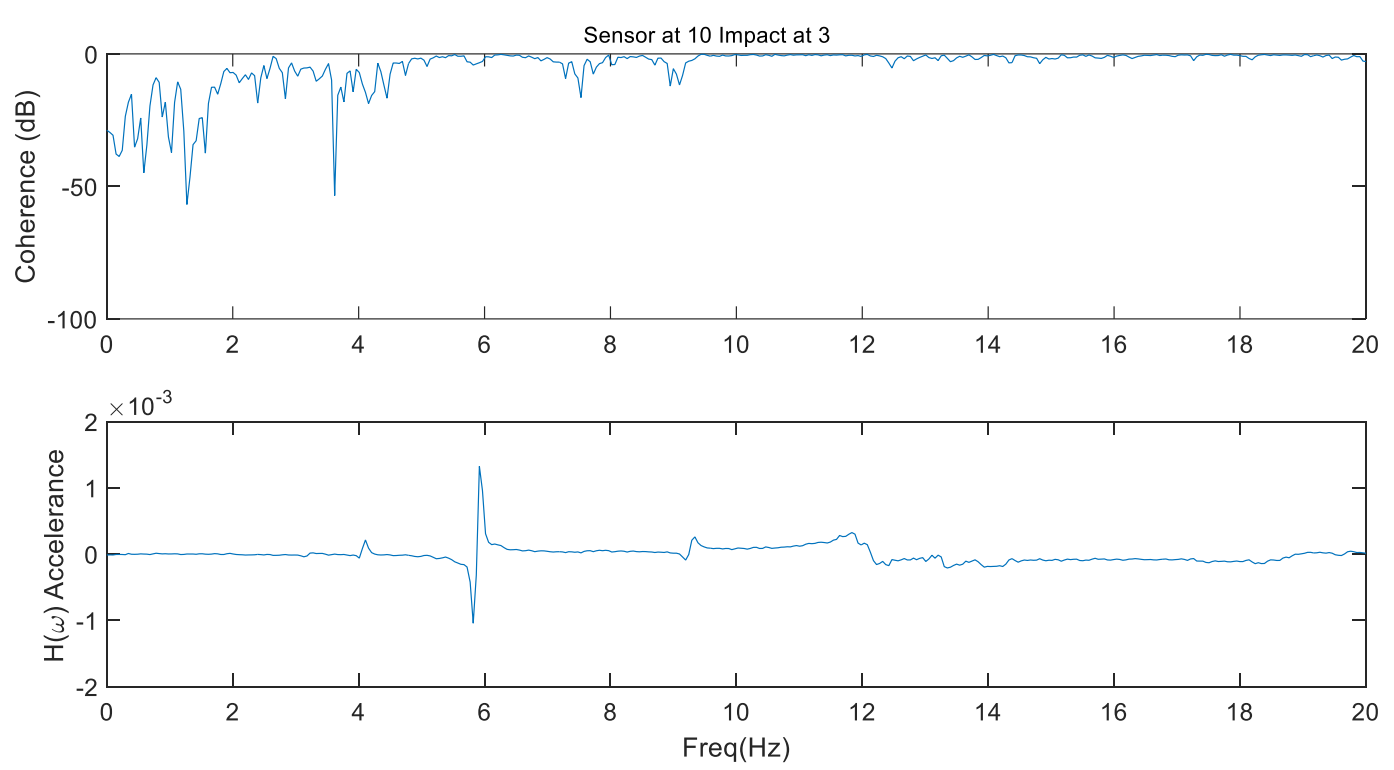

Figure 37. FRF from impact at location 3 and measurement at location 10 

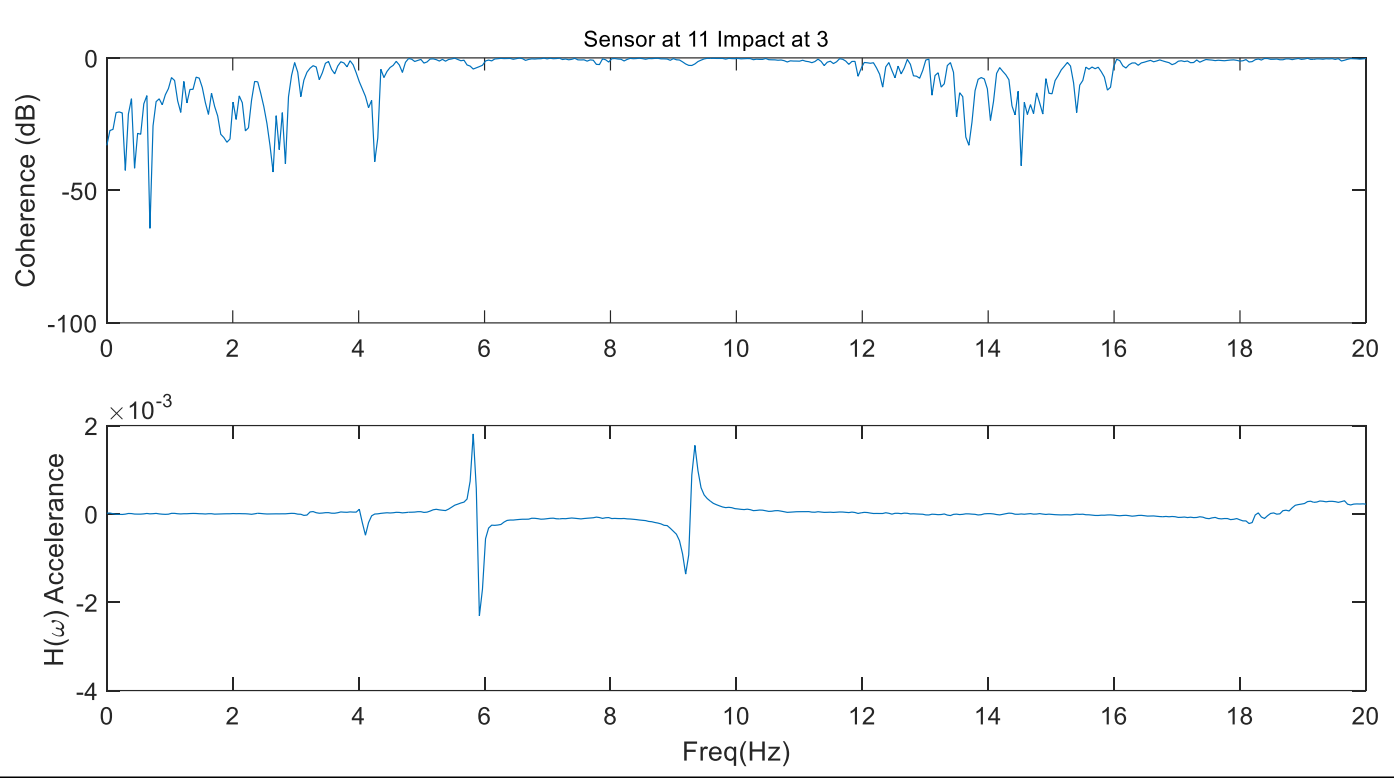

Figure 38. FRF from impact at location 3 and measurement at location 11
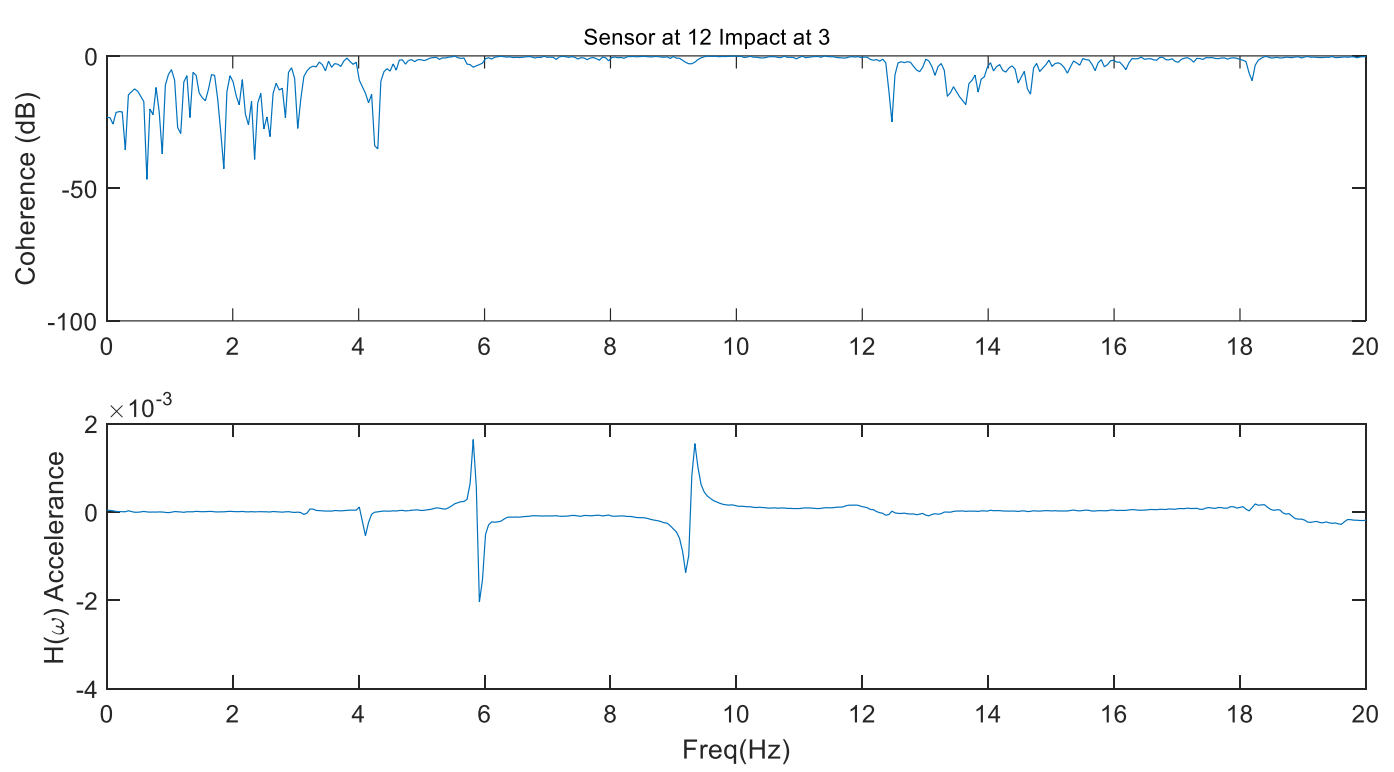

Figure 39. FRF from impact at location 3 and measurement at location 12 

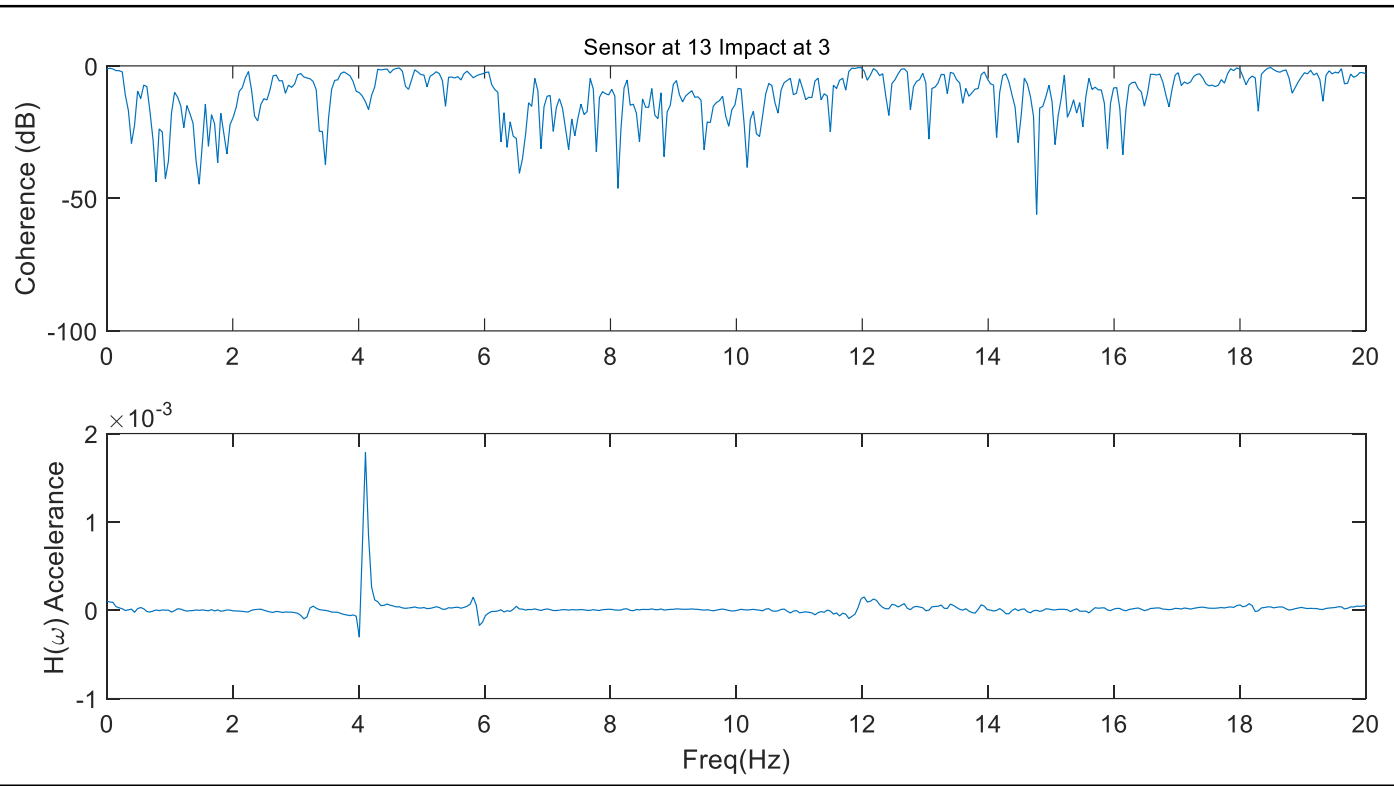

Figure 40. FRF from impact at location 3 and measurement at location 13
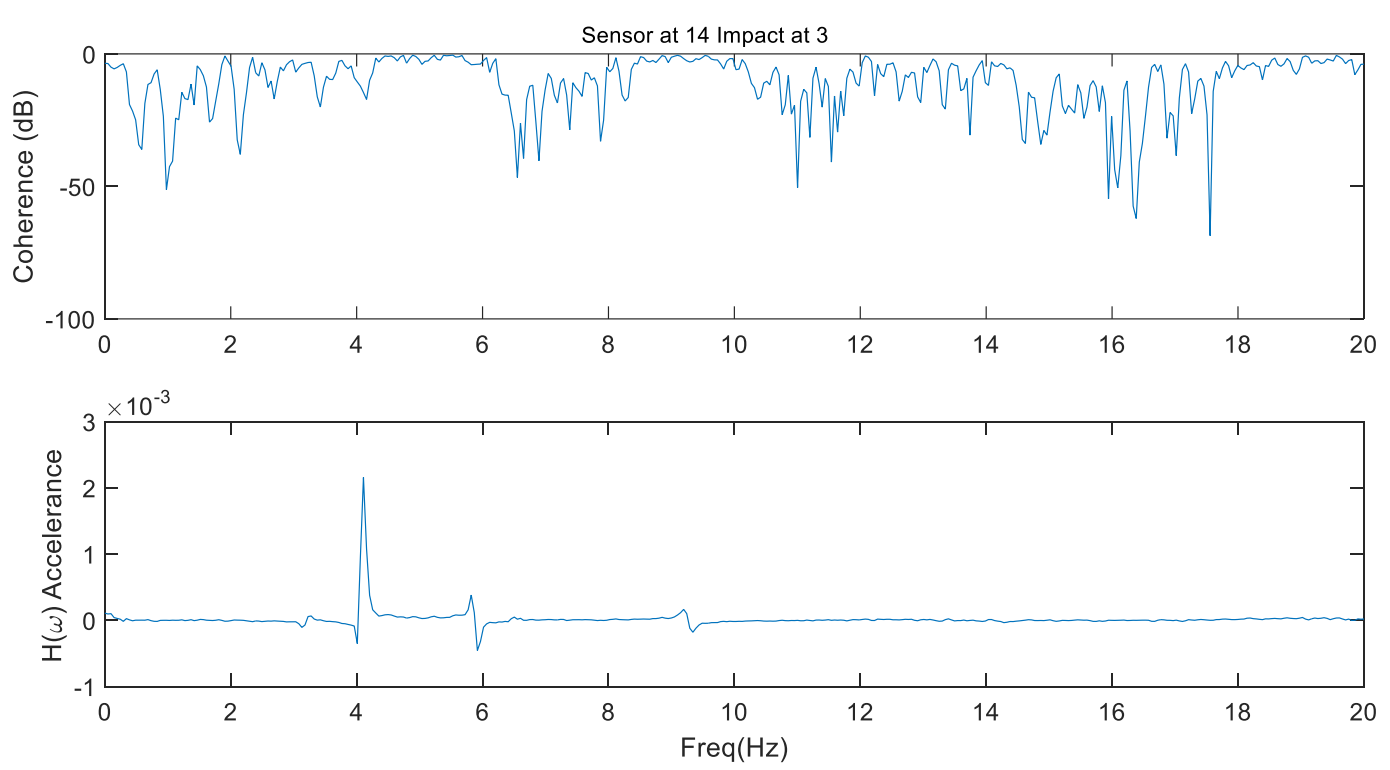

Figure 41. FRF from impact at location 3 and measurement at location 14 

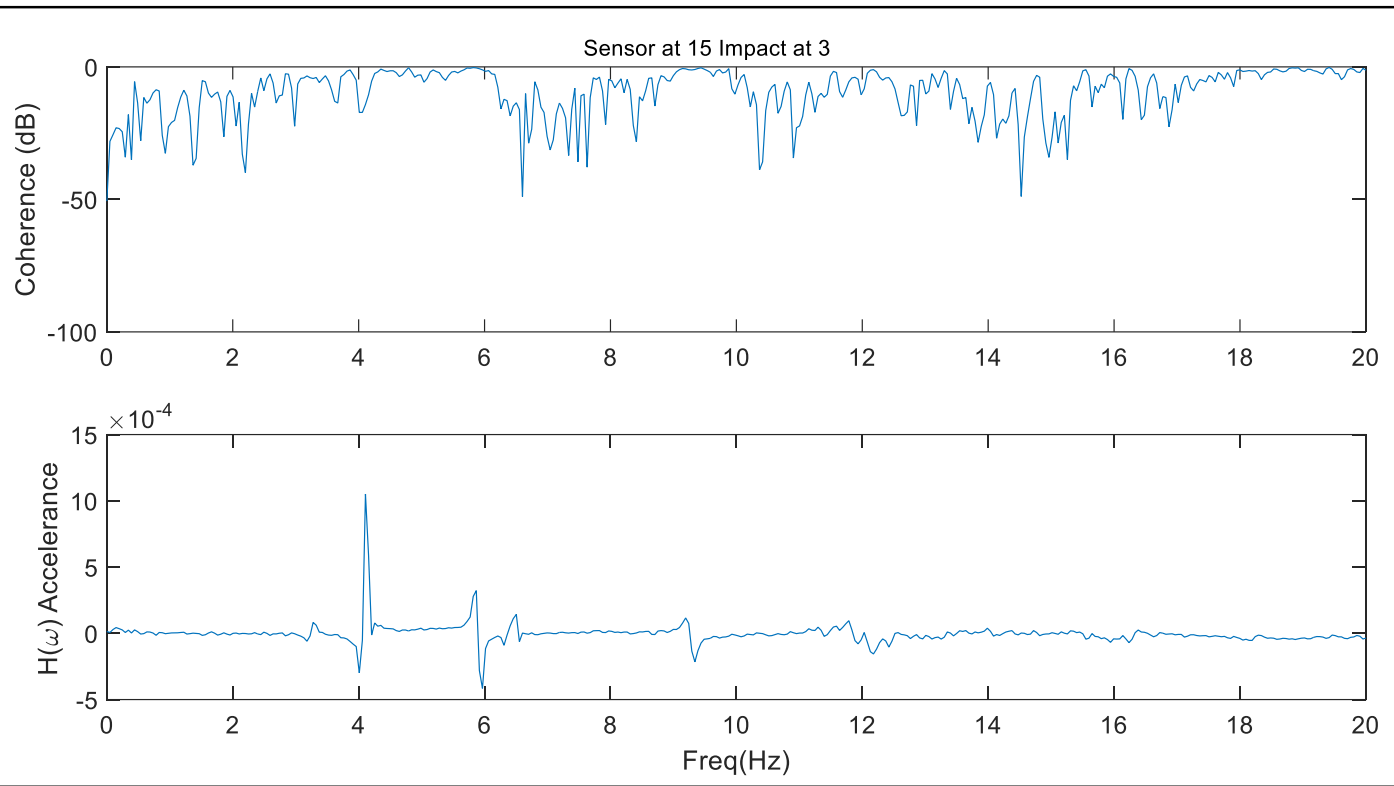

Figure 42. FRF from impact at location 3 and measurement at location 15
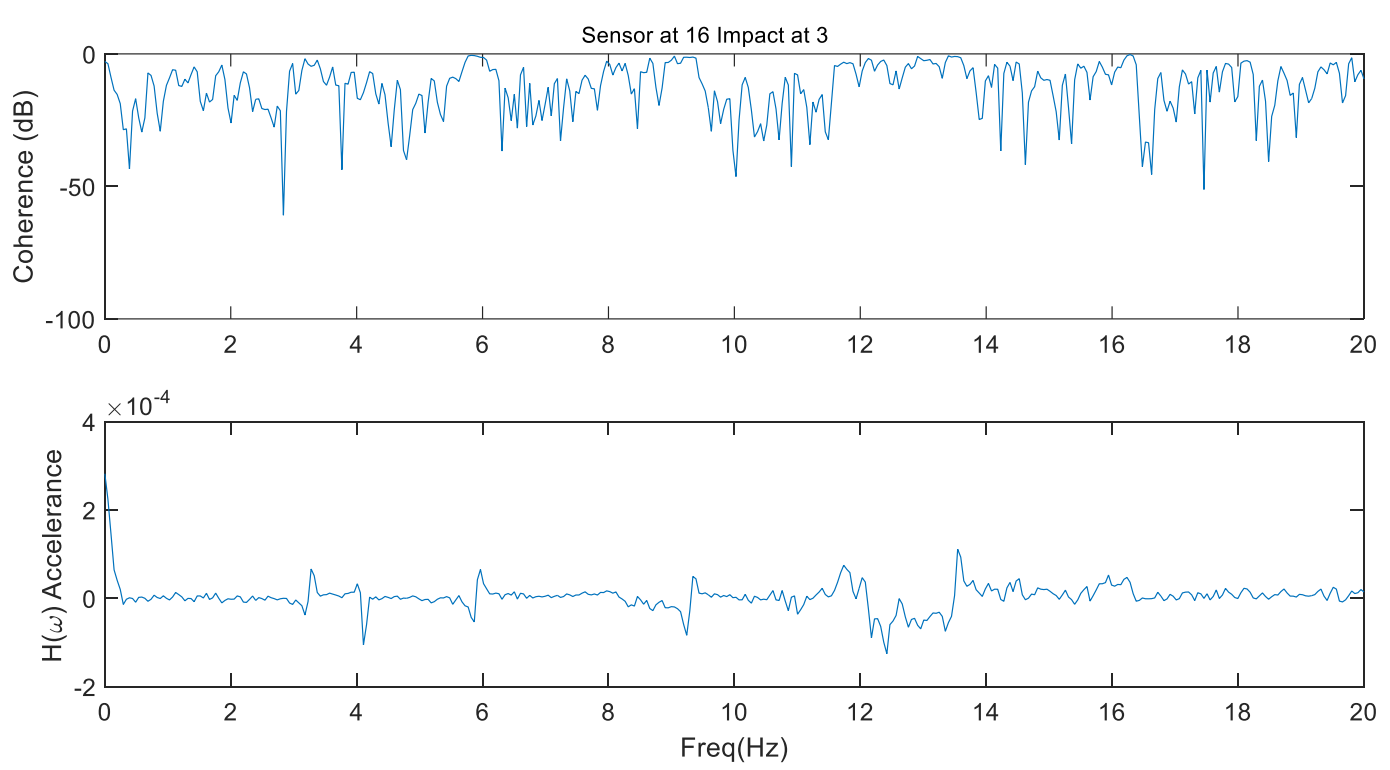

Figure 43. FRF from impact at location 3 and measurement at location 16 

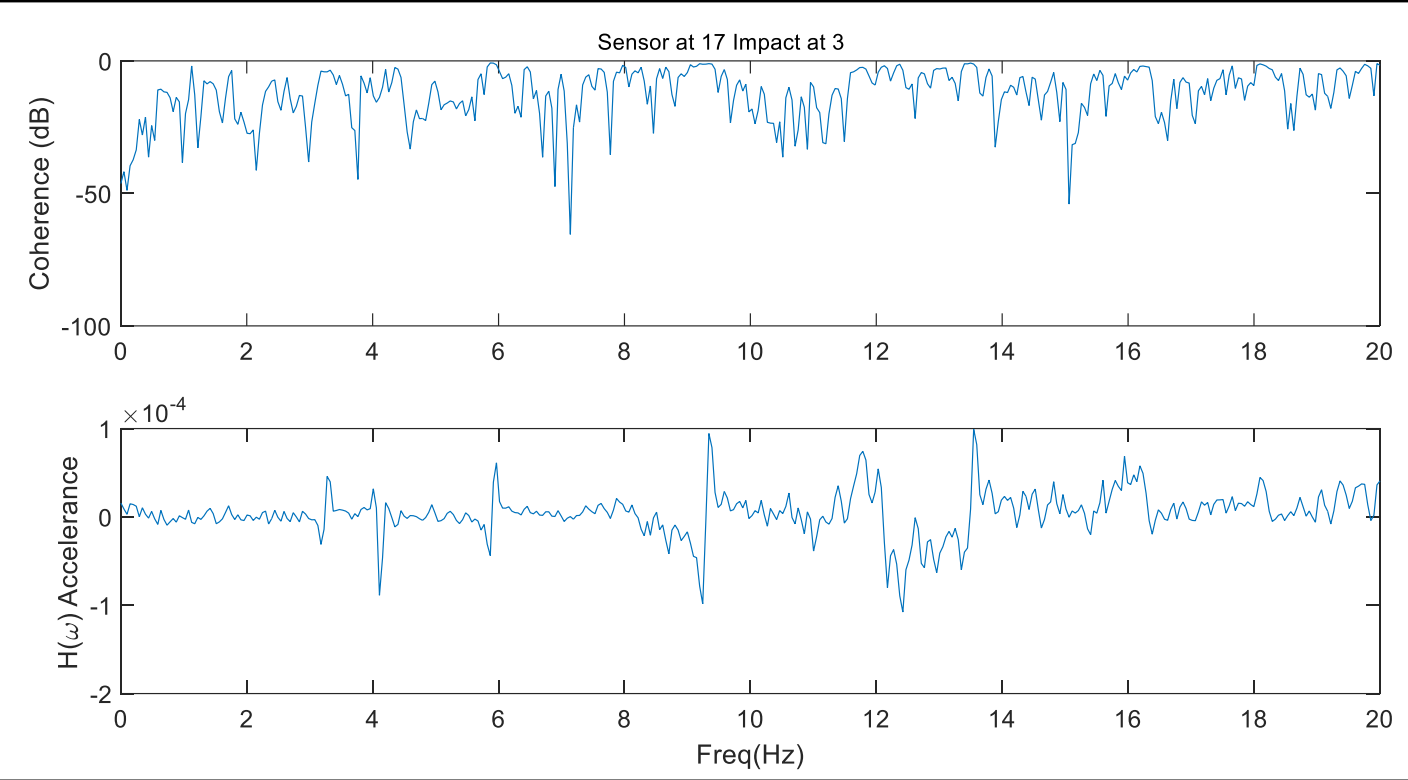

Figure 44. FRF from impact at location 3 and measurement at location 17
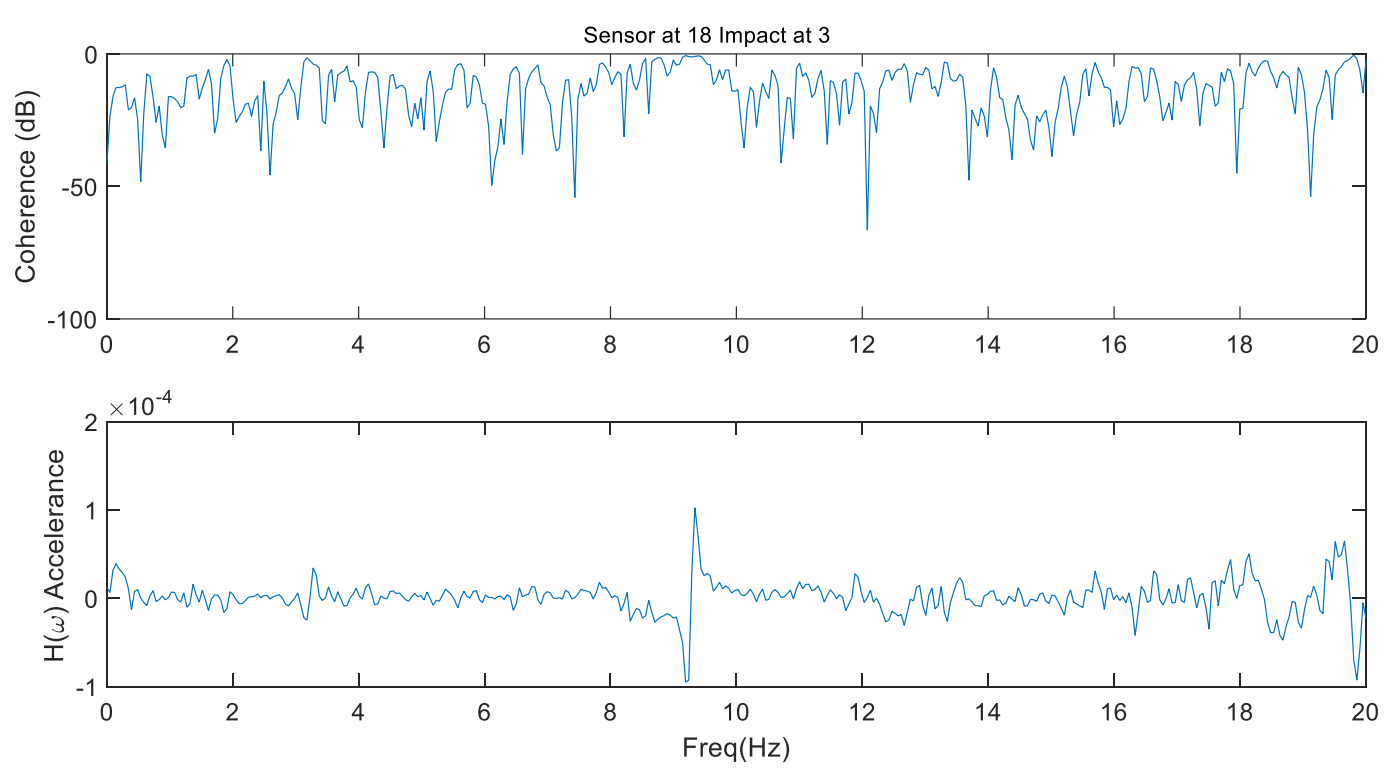

Figure 45. FRF from impact at location 3 and measurement at location 18 

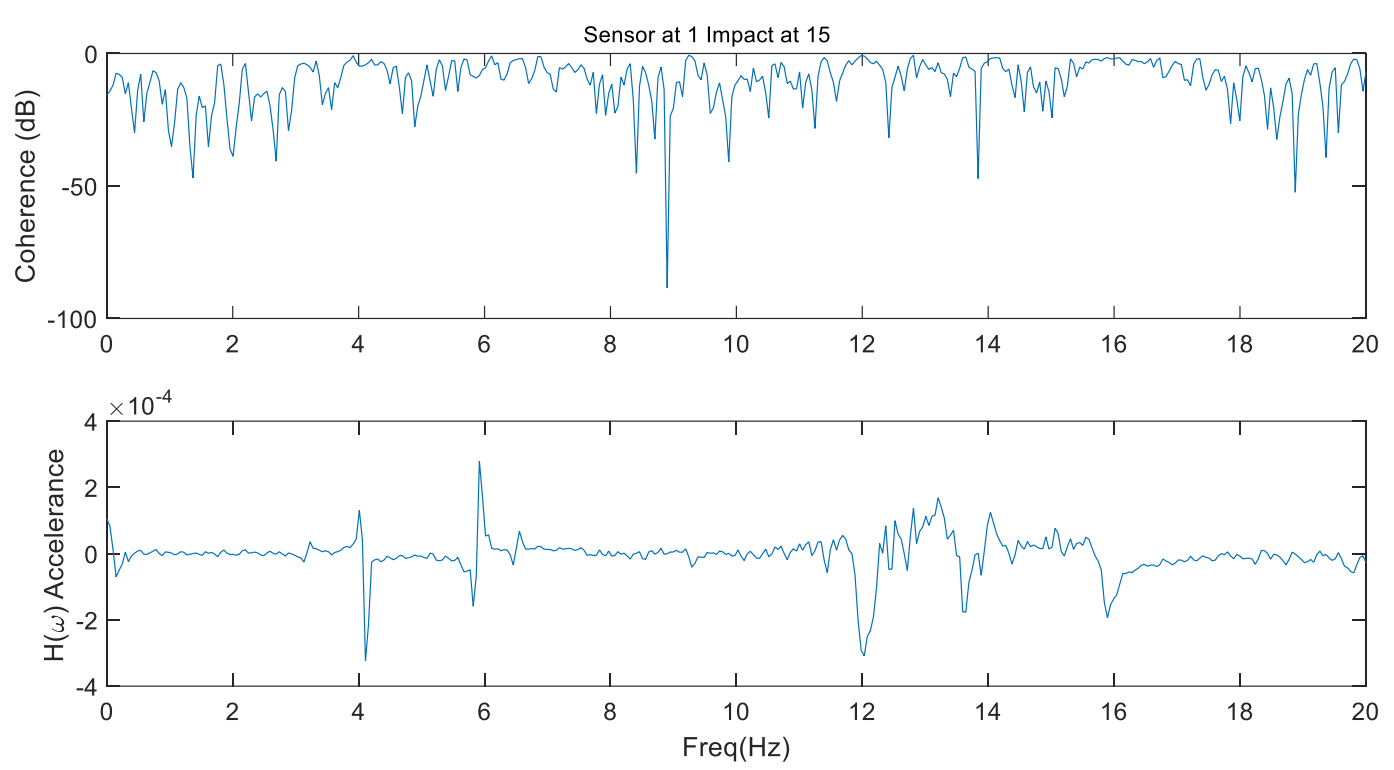

Figure 46. FRF from impact at location 15 and measurement at location 1
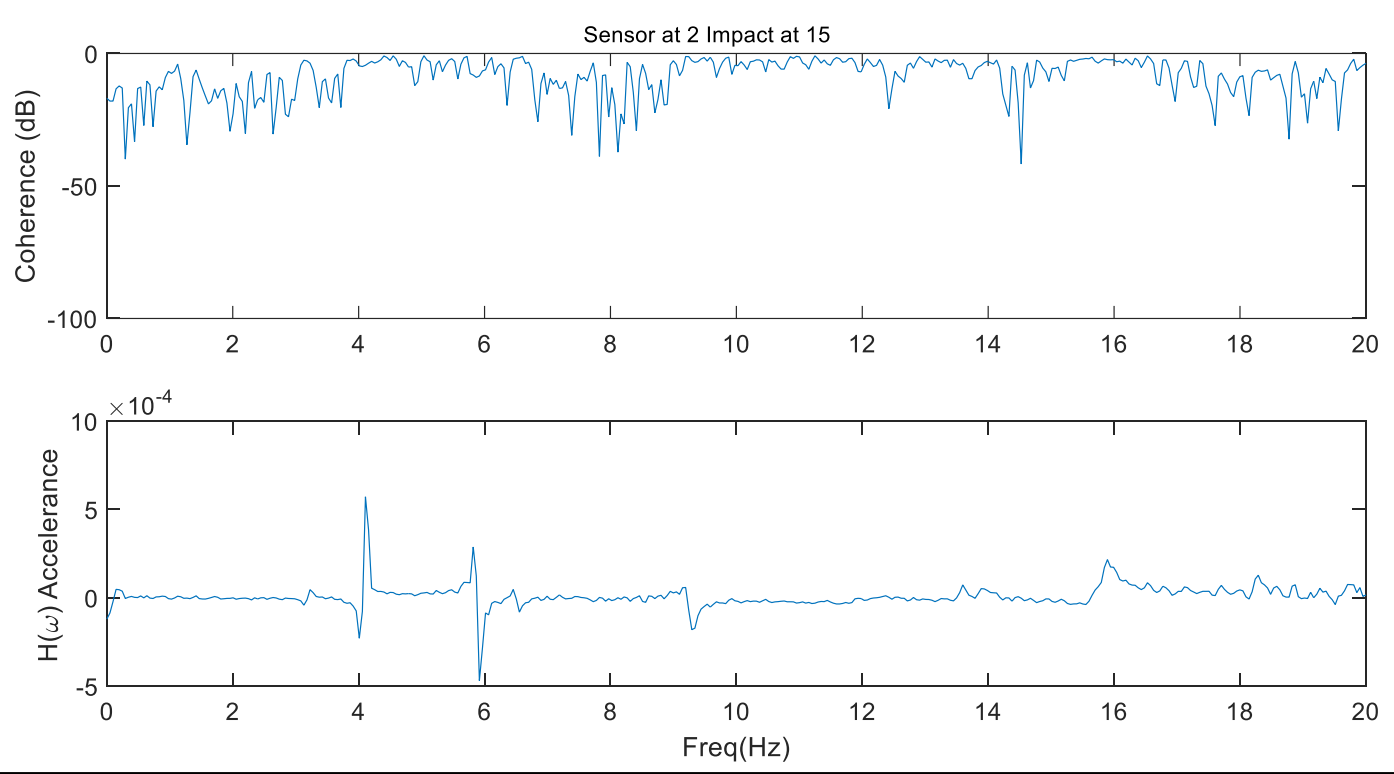

Figure 47. FRF from impact at location 15 and measurement at location 2 

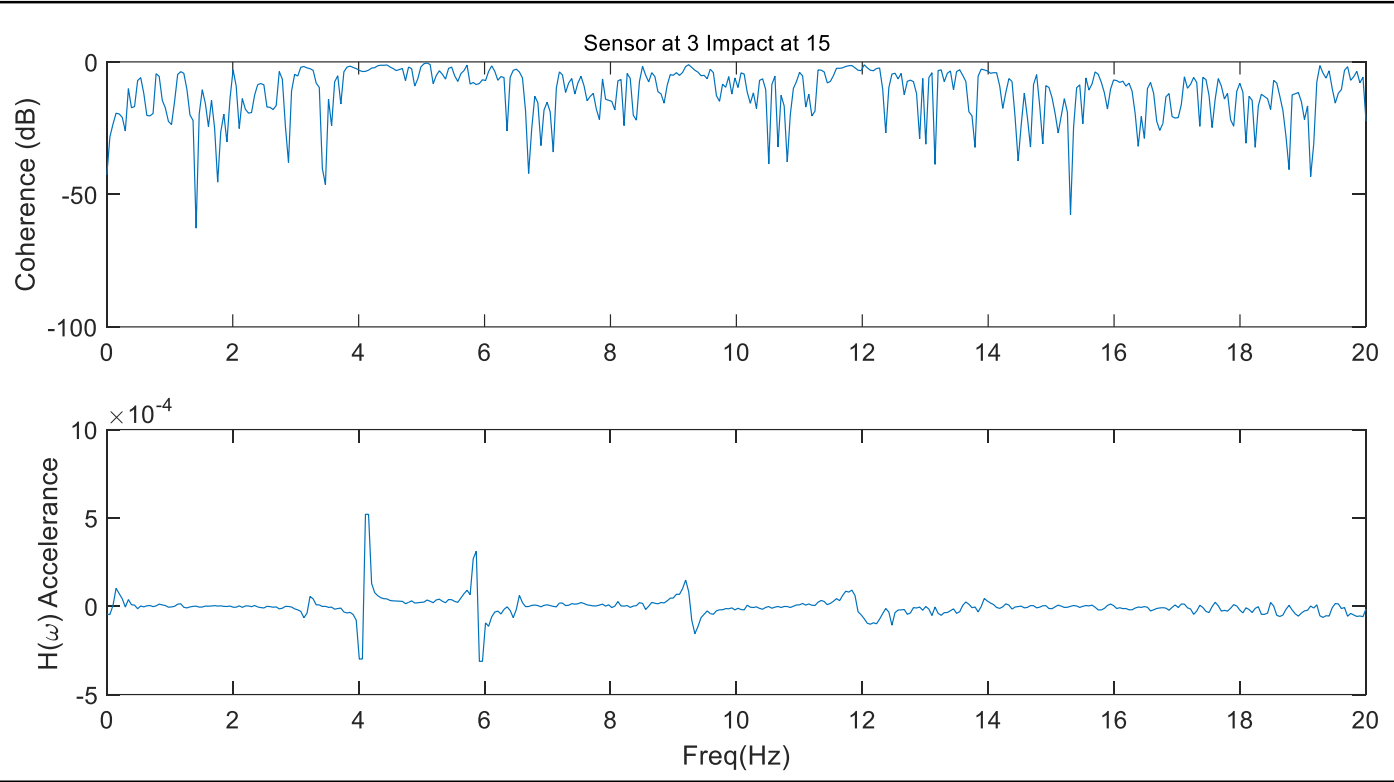

Figure 48. FRF from impact at location 15 and measurement at location 3
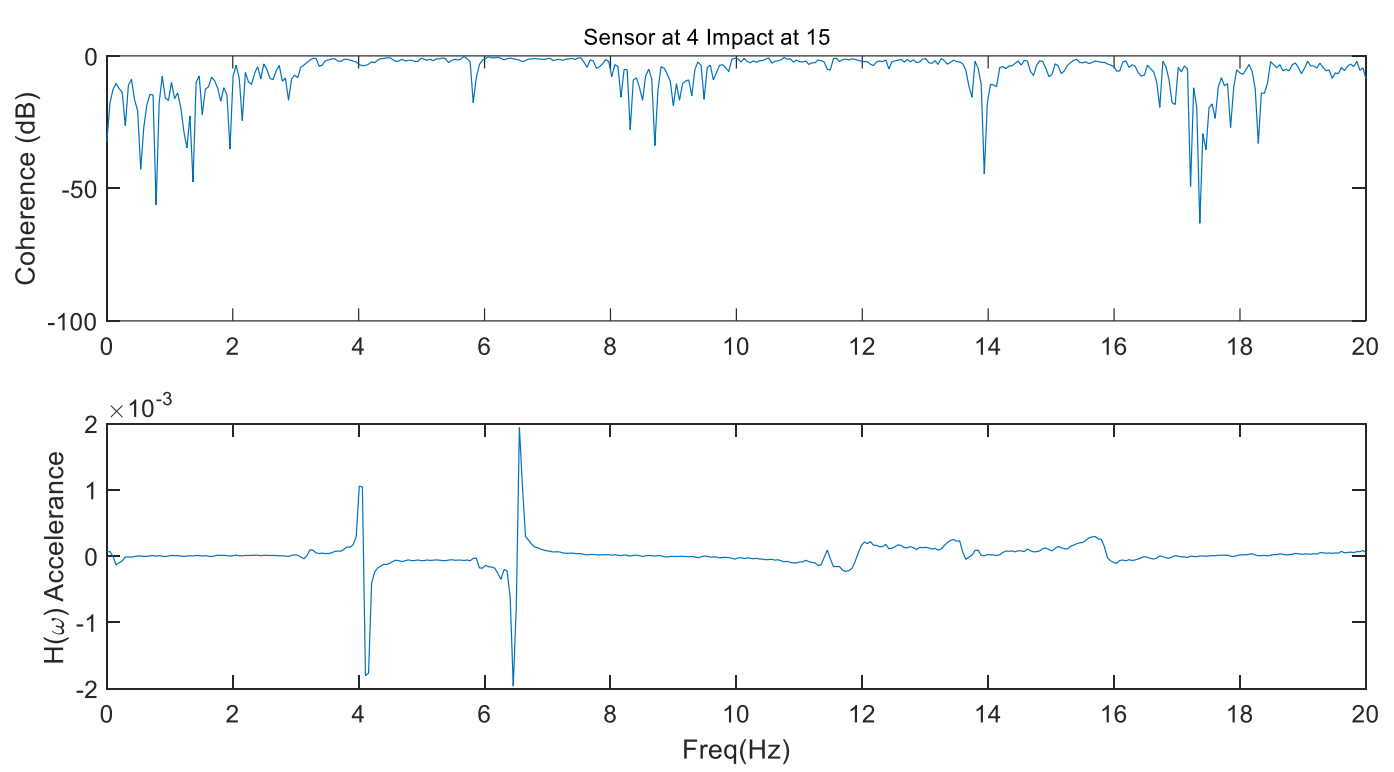

Figure 49. FRF from impact at location 15 and measurement at location 4 

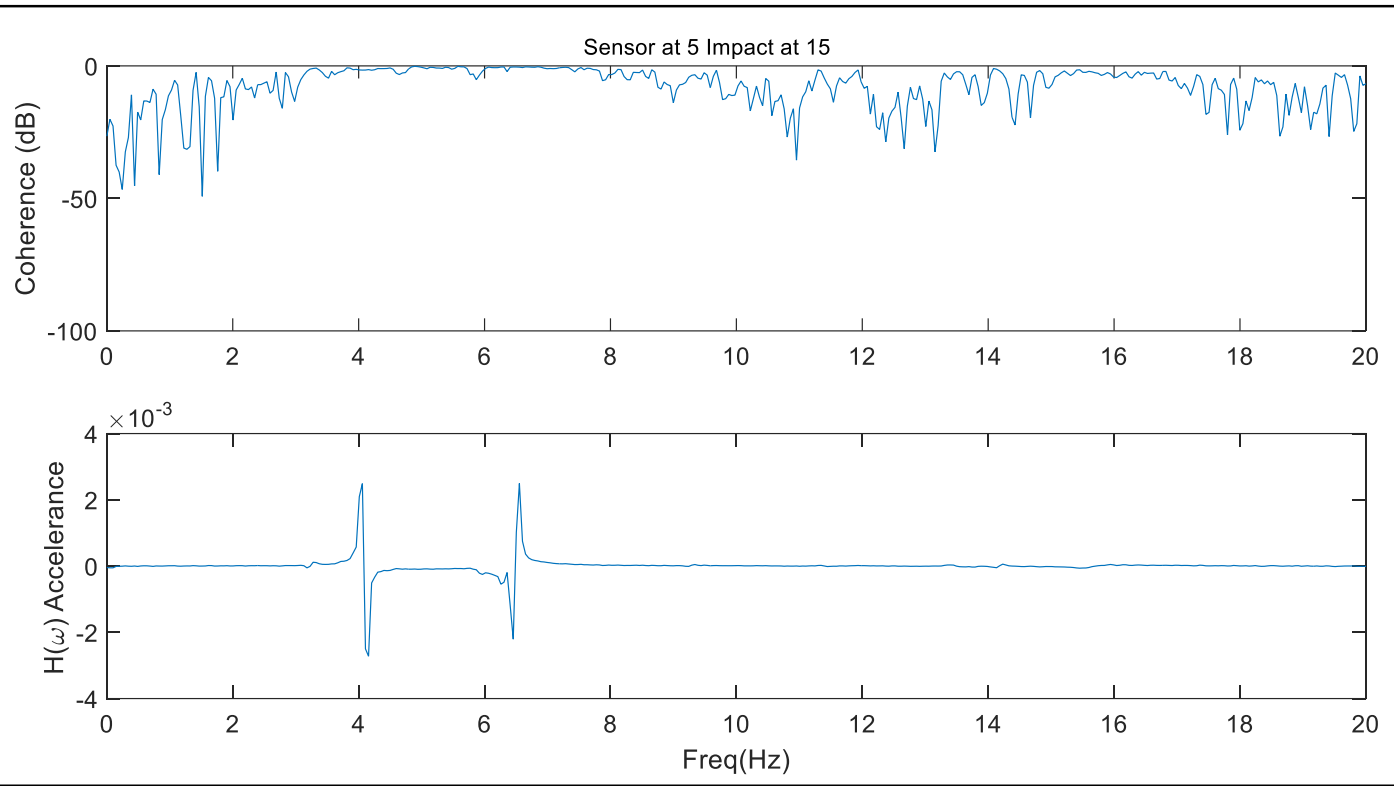

Figure 50. FRF from impact at location 15 and measurement at location 5
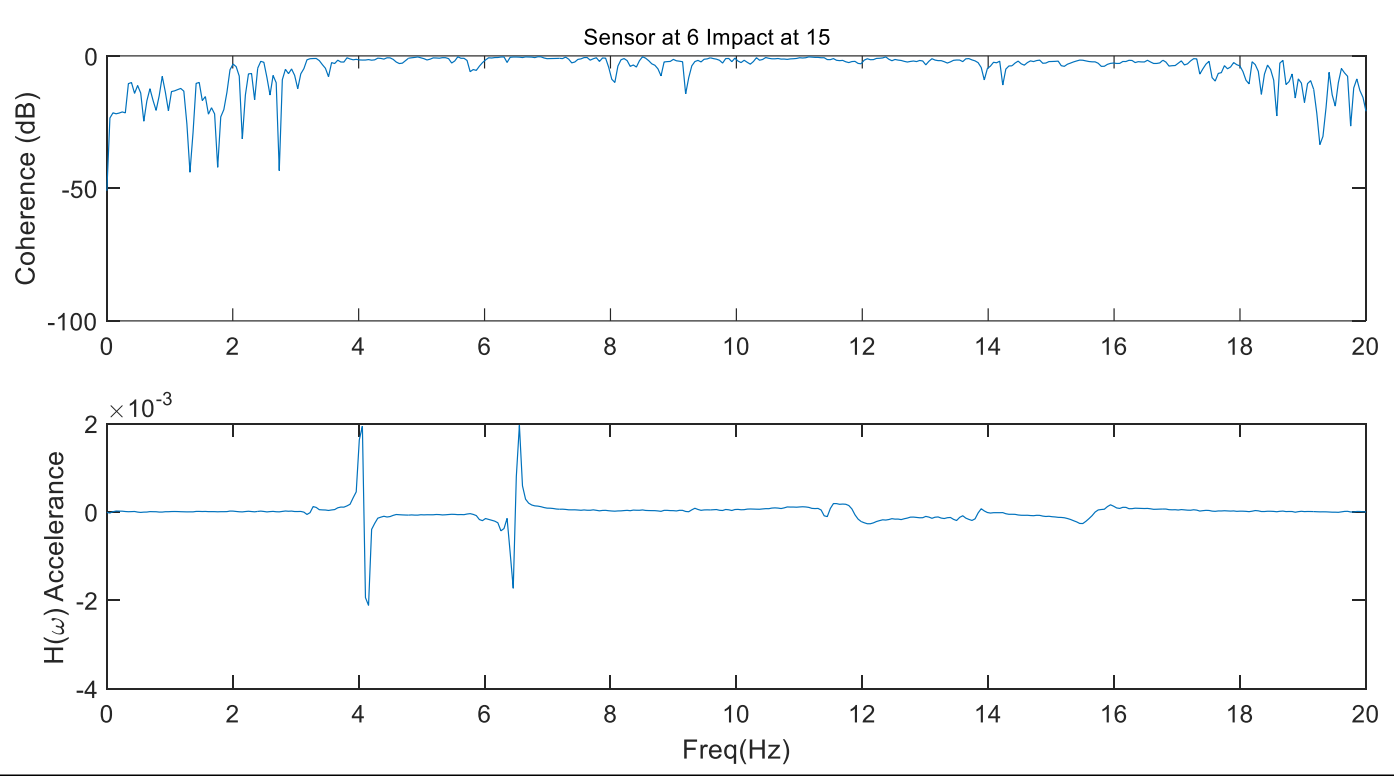

Figure 51. FRF from impact at location 15 and measurement at location 6 

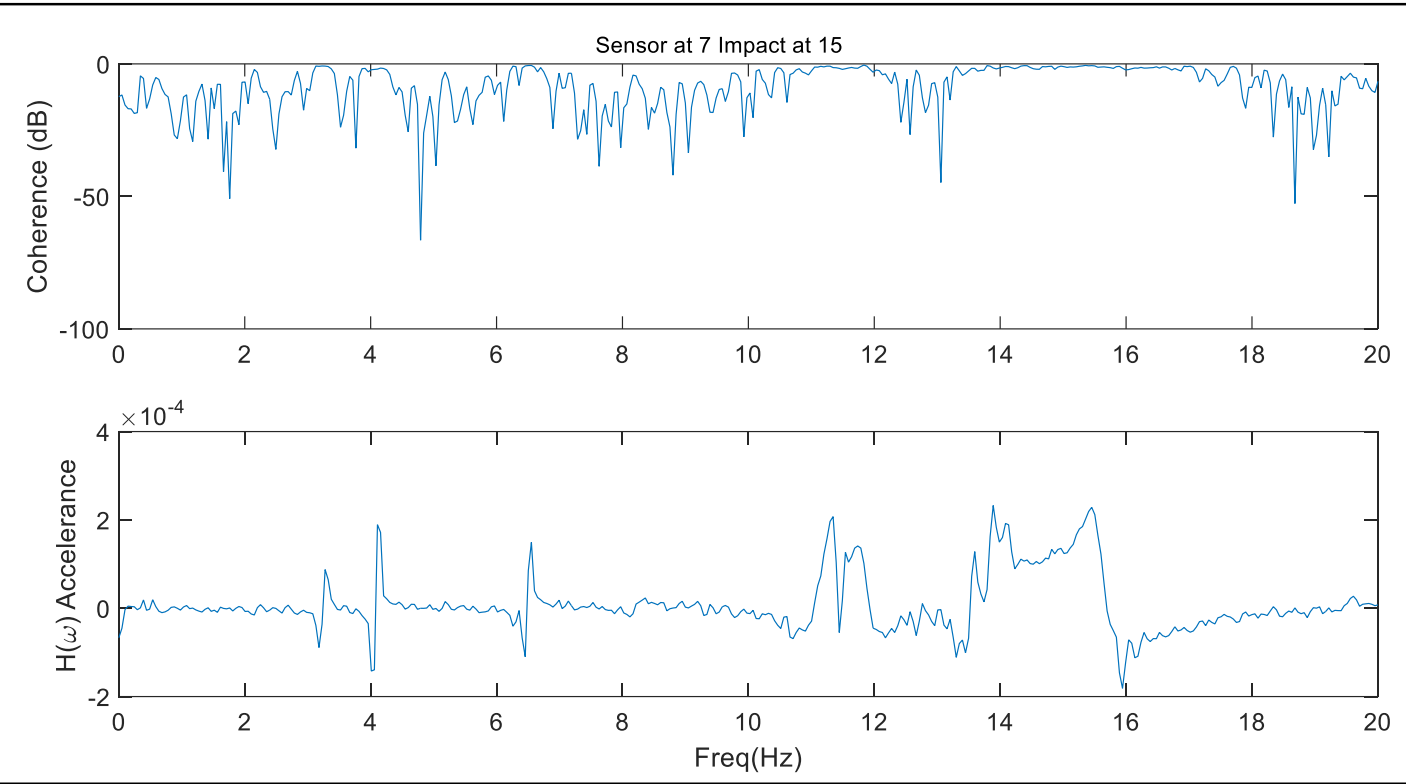

Figure 52. FRF from impact at location 15 and measurement at location 7
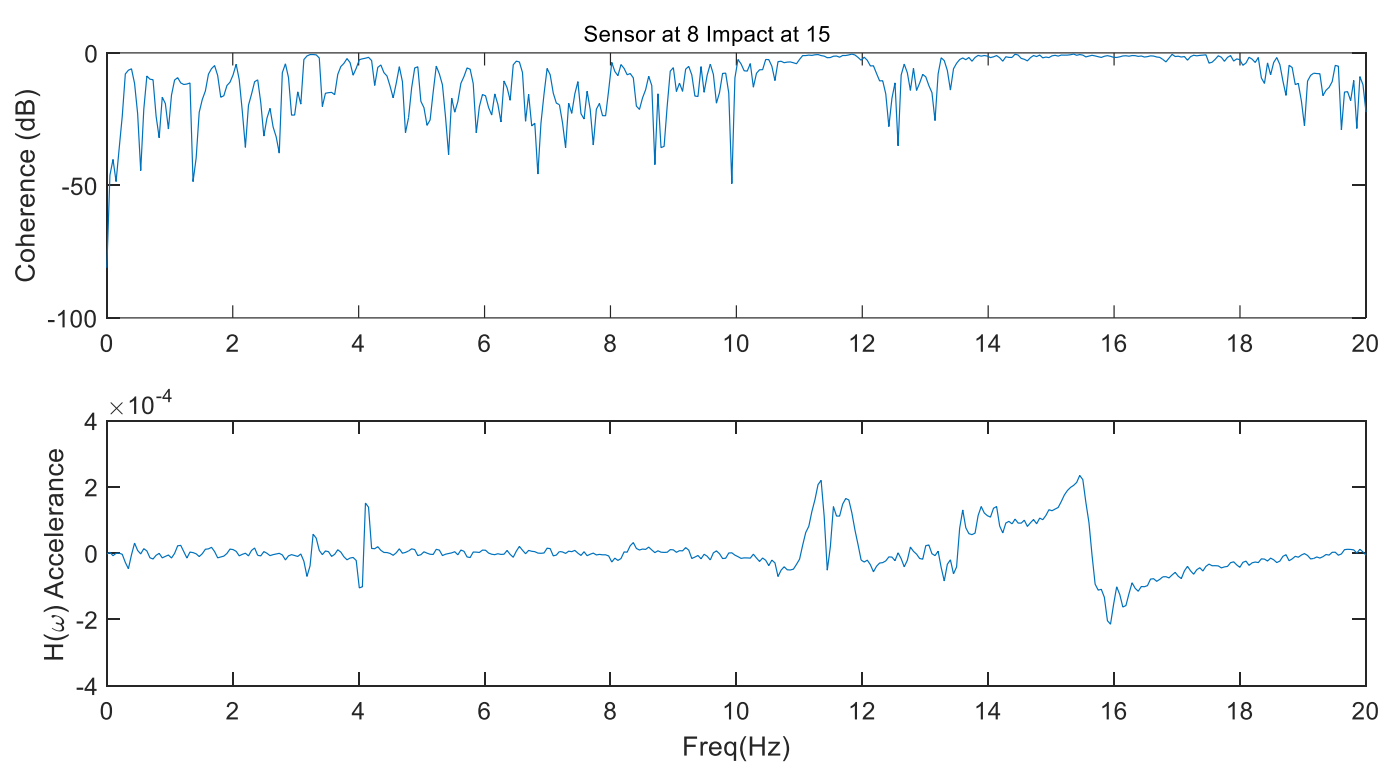

Figure 53. FRF from impact at location 15 and measurement at location 8 

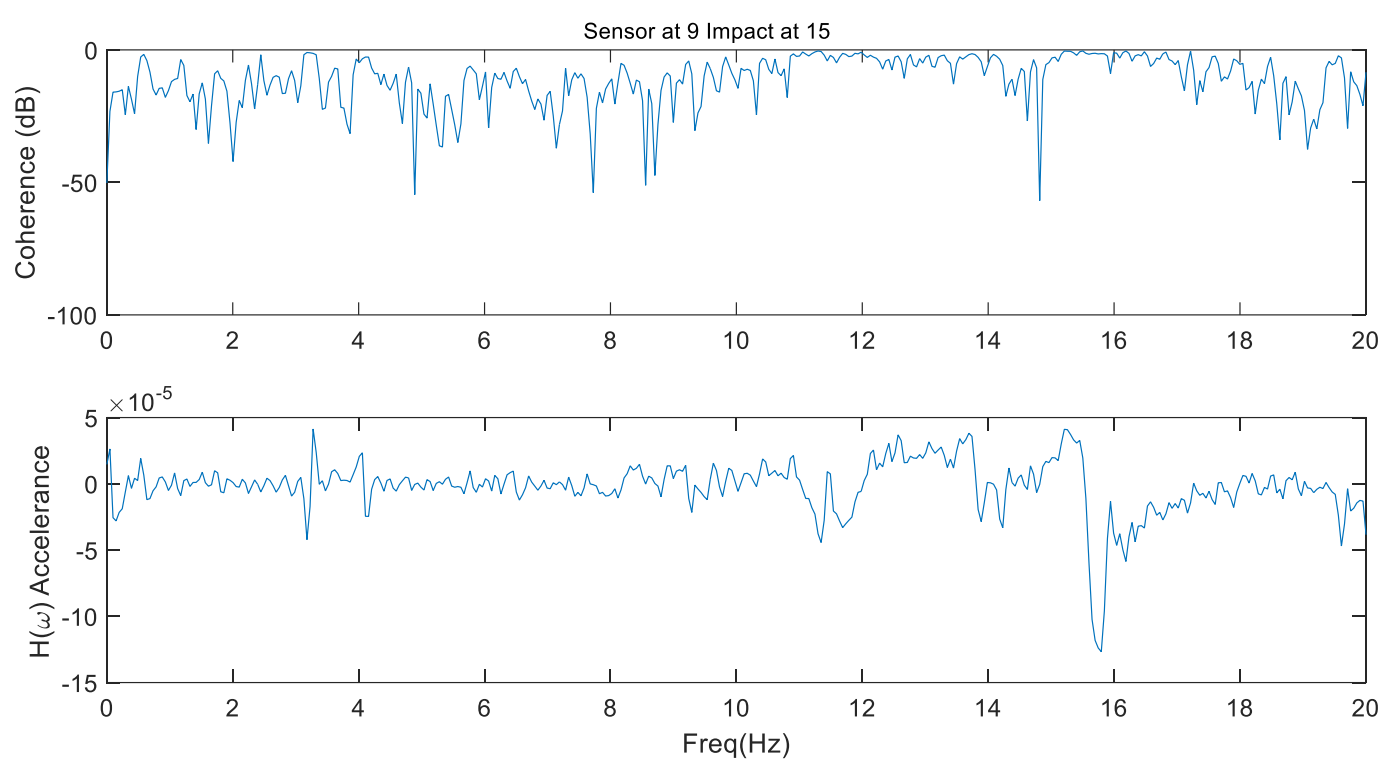

Figure 54. FRF from impact at location 15 and measurement at location 9
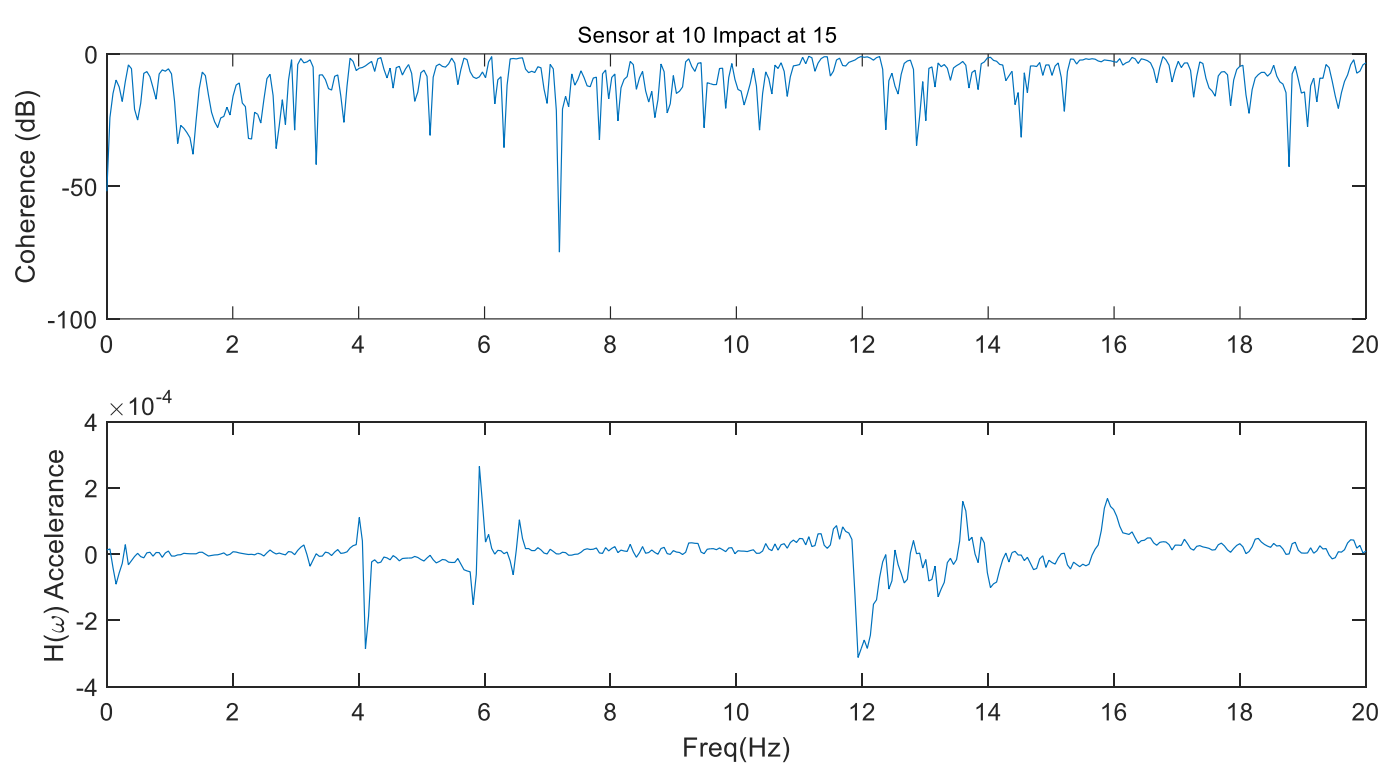

Figure 55. FRF from impact at location 15 and measurement at location 10 

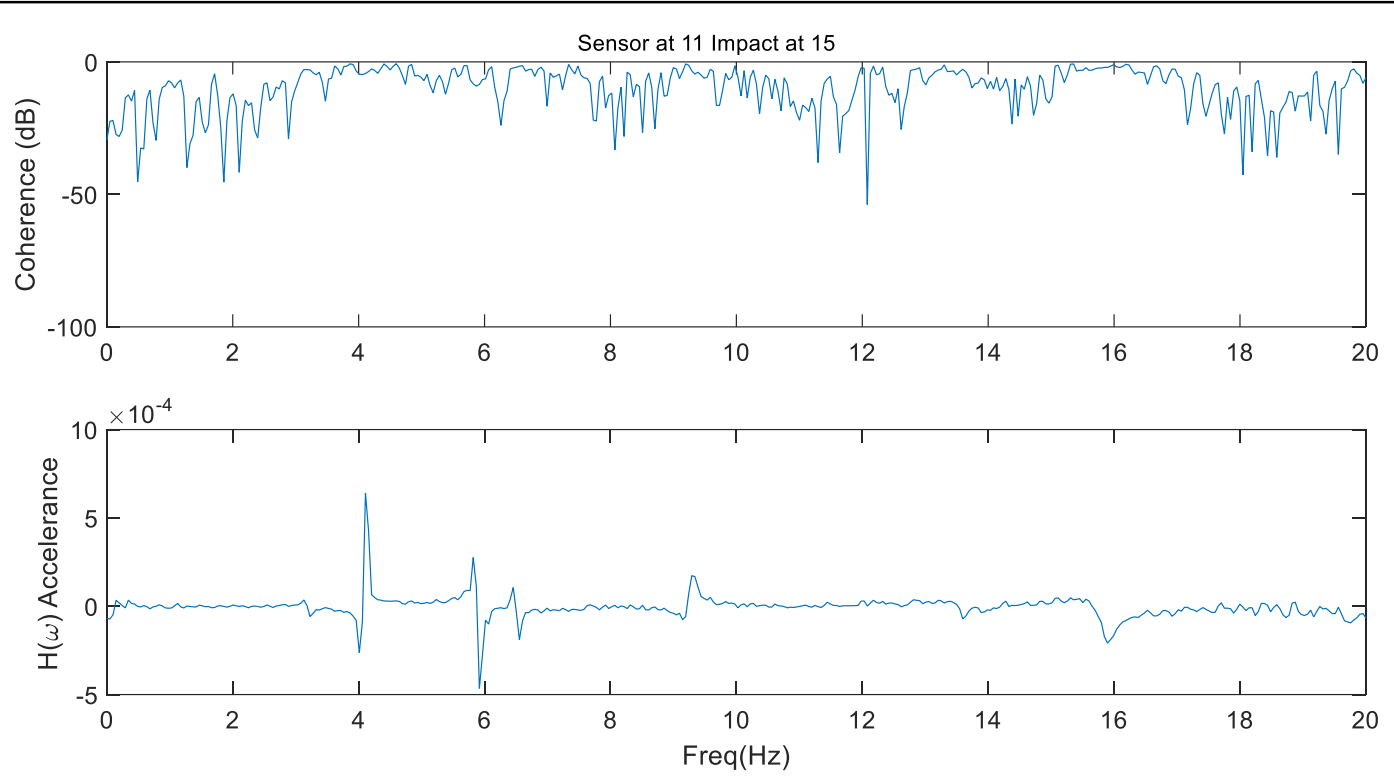

Figure 56. FRF from impact at location 15 and measurement at location 11
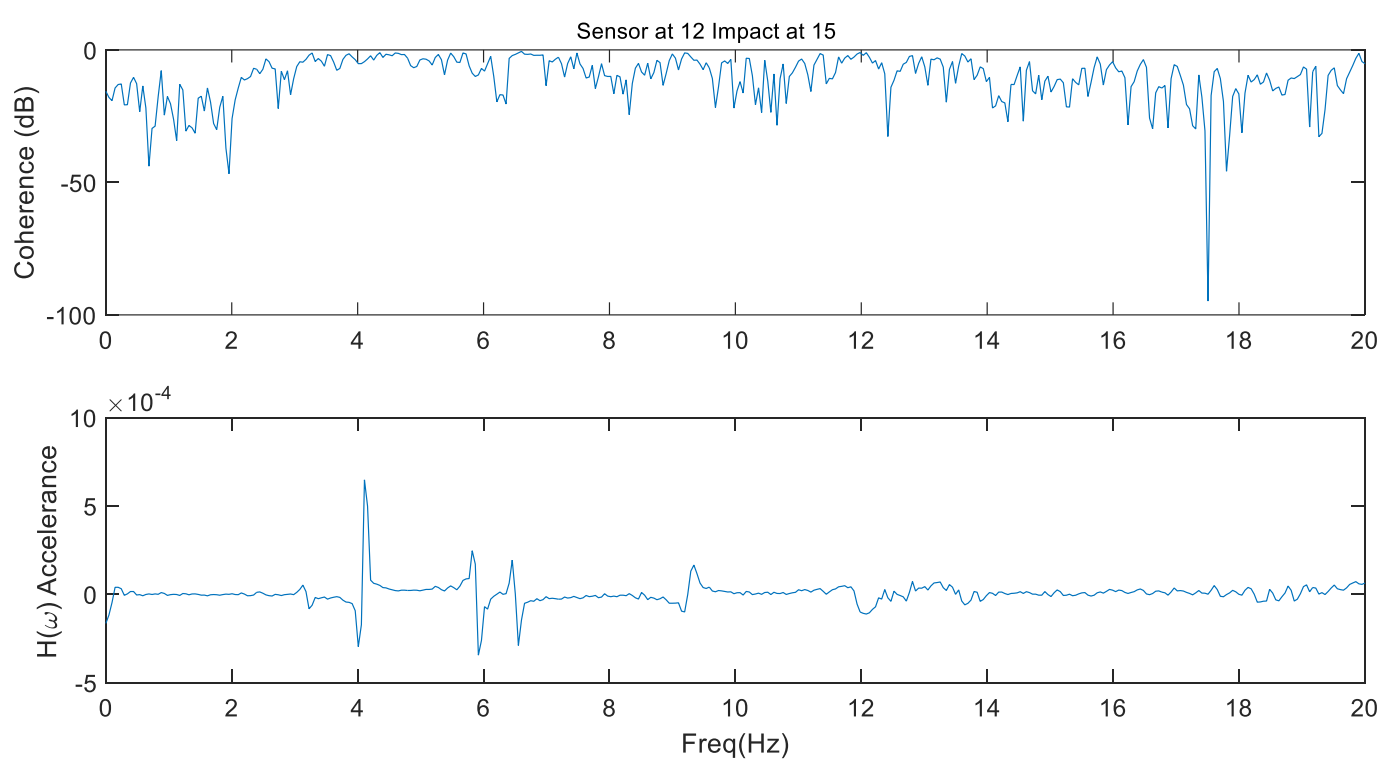

Figure 57. FRF from impact at location 15 and measurement at location 12 

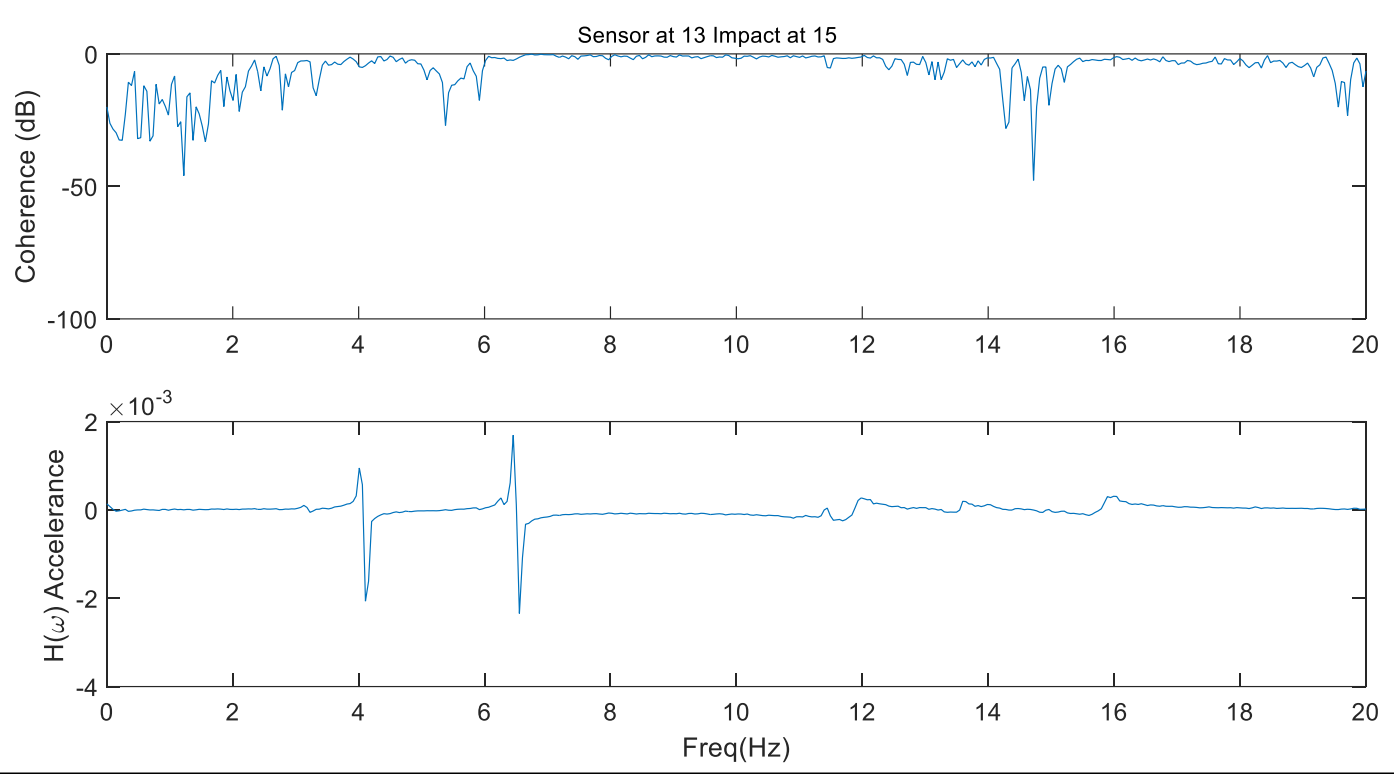

Figure 58. FRF from impact at location 15 and measurement at location 13
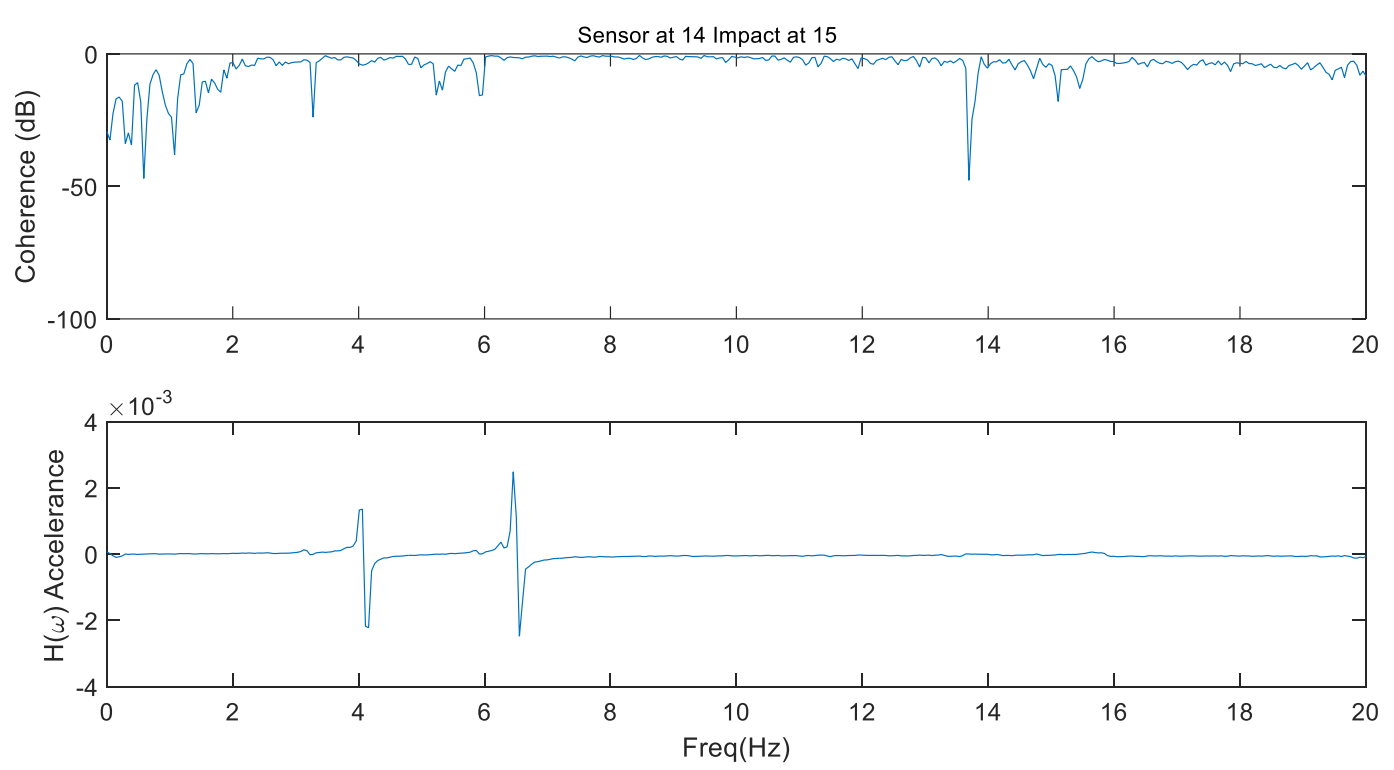

Figure 59. FRF from impact at location 15 and measurement at location 14 

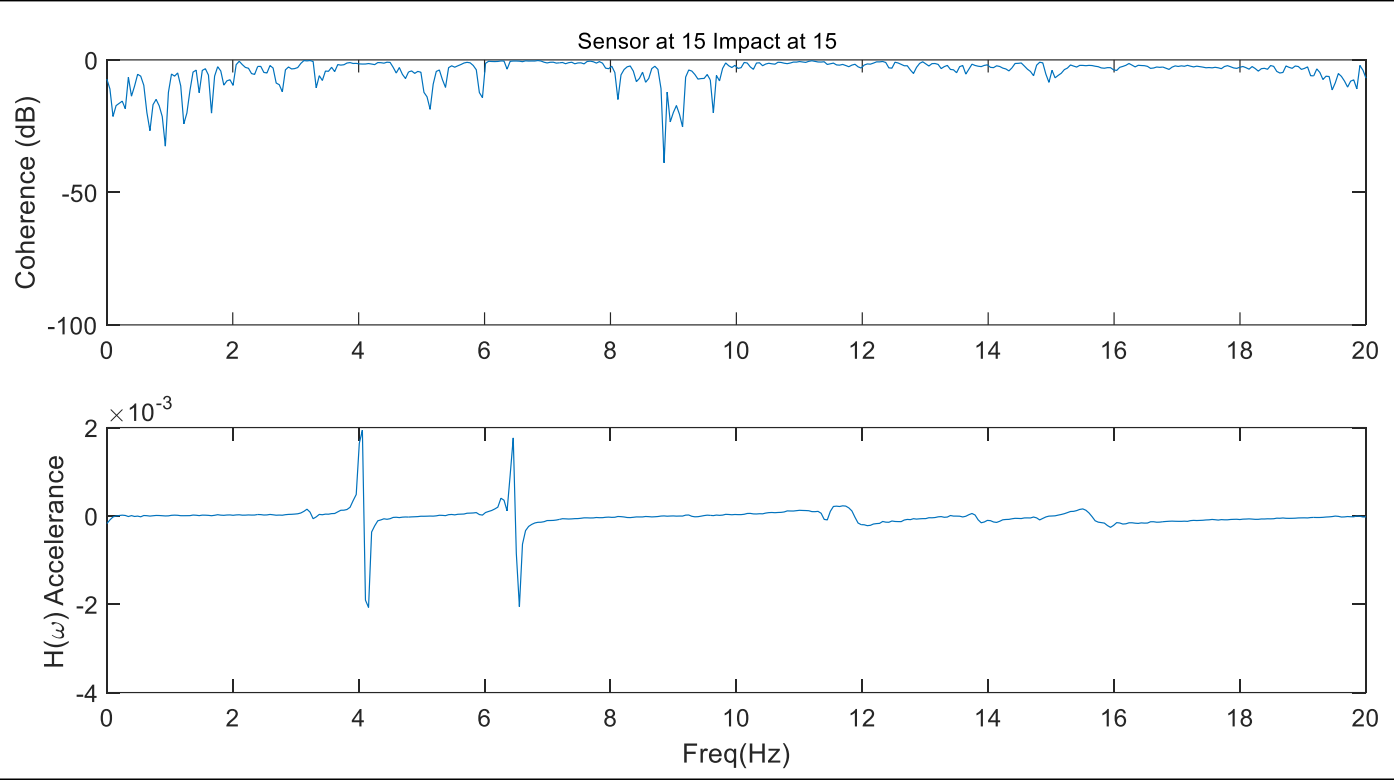

Figure 60. FRF from impact at location 15 and measurement at location 15
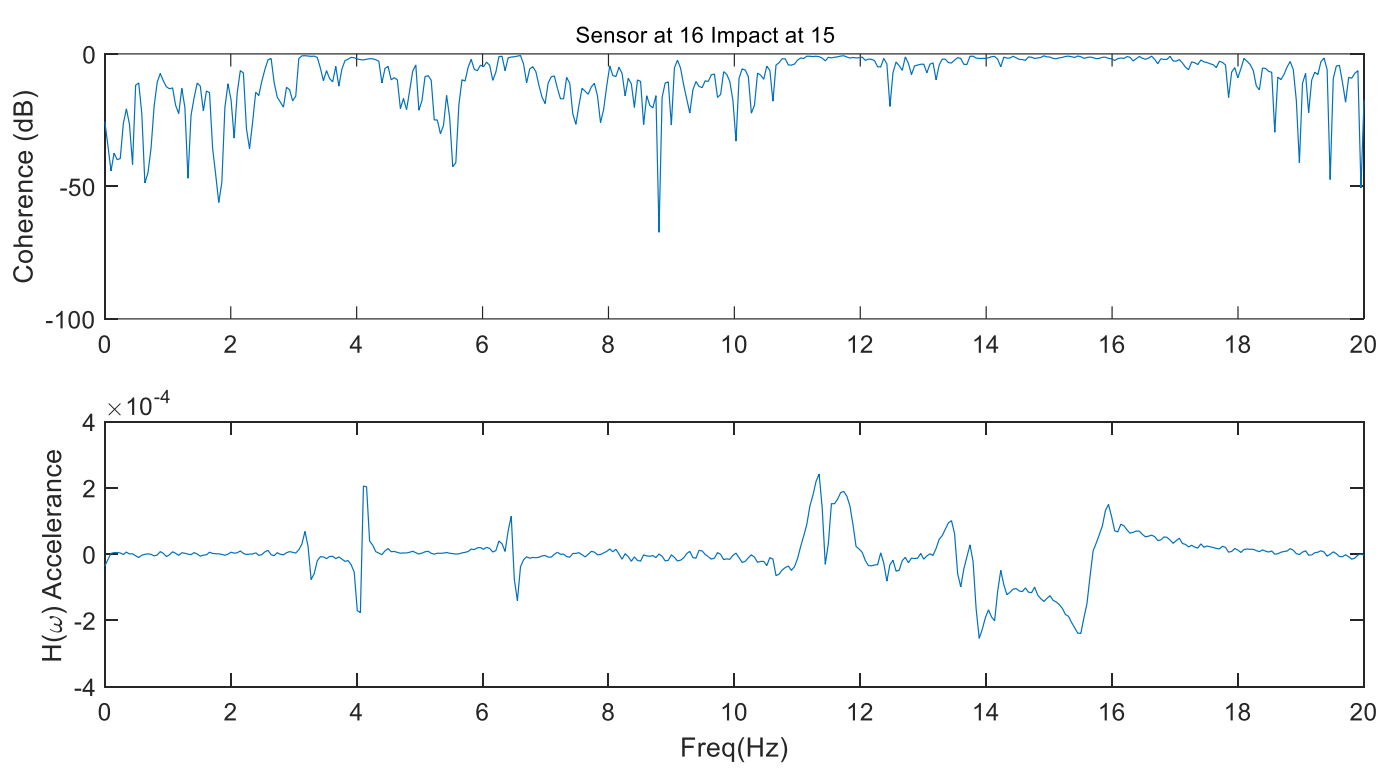

Figure 61. FRF from impact at location 15 and measurement at location 16 

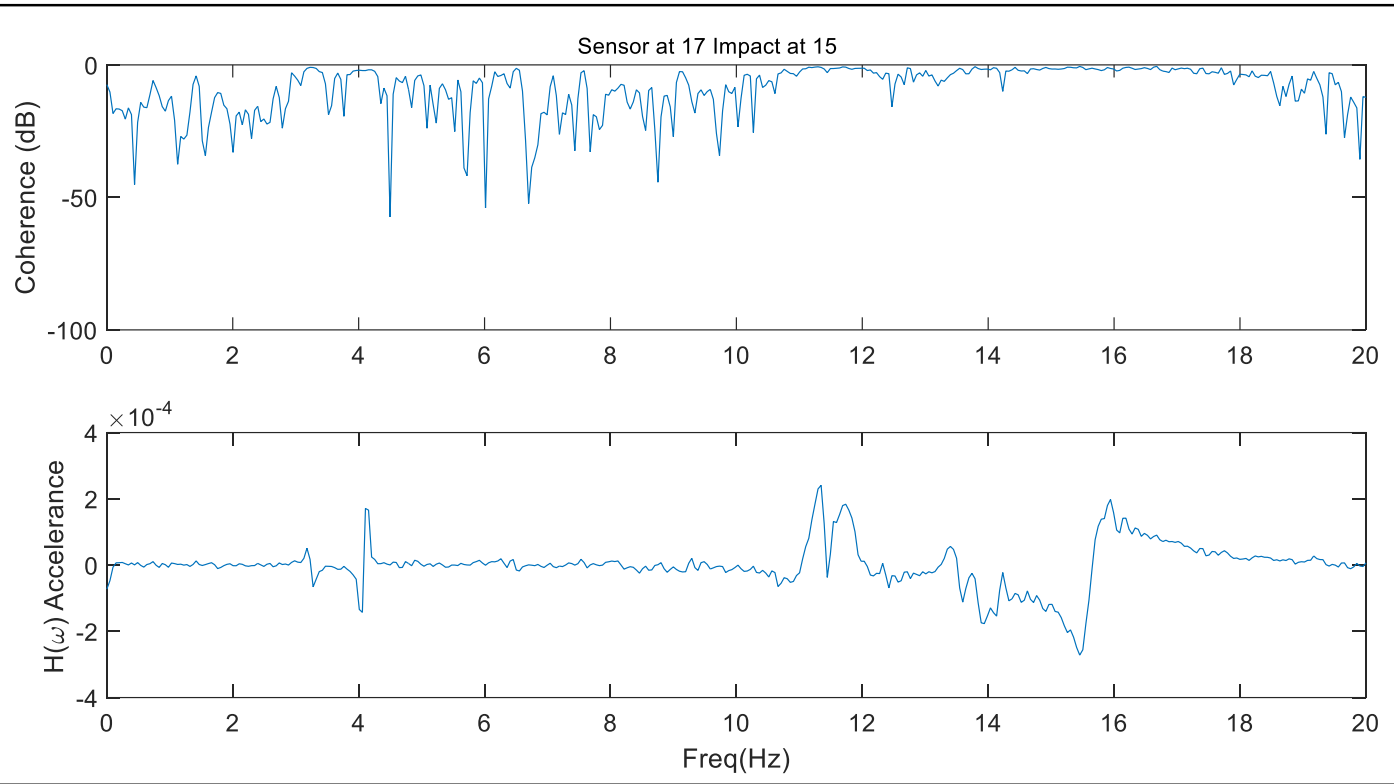

Figure 62. FRF from impact at location 15 and measurement at location 17
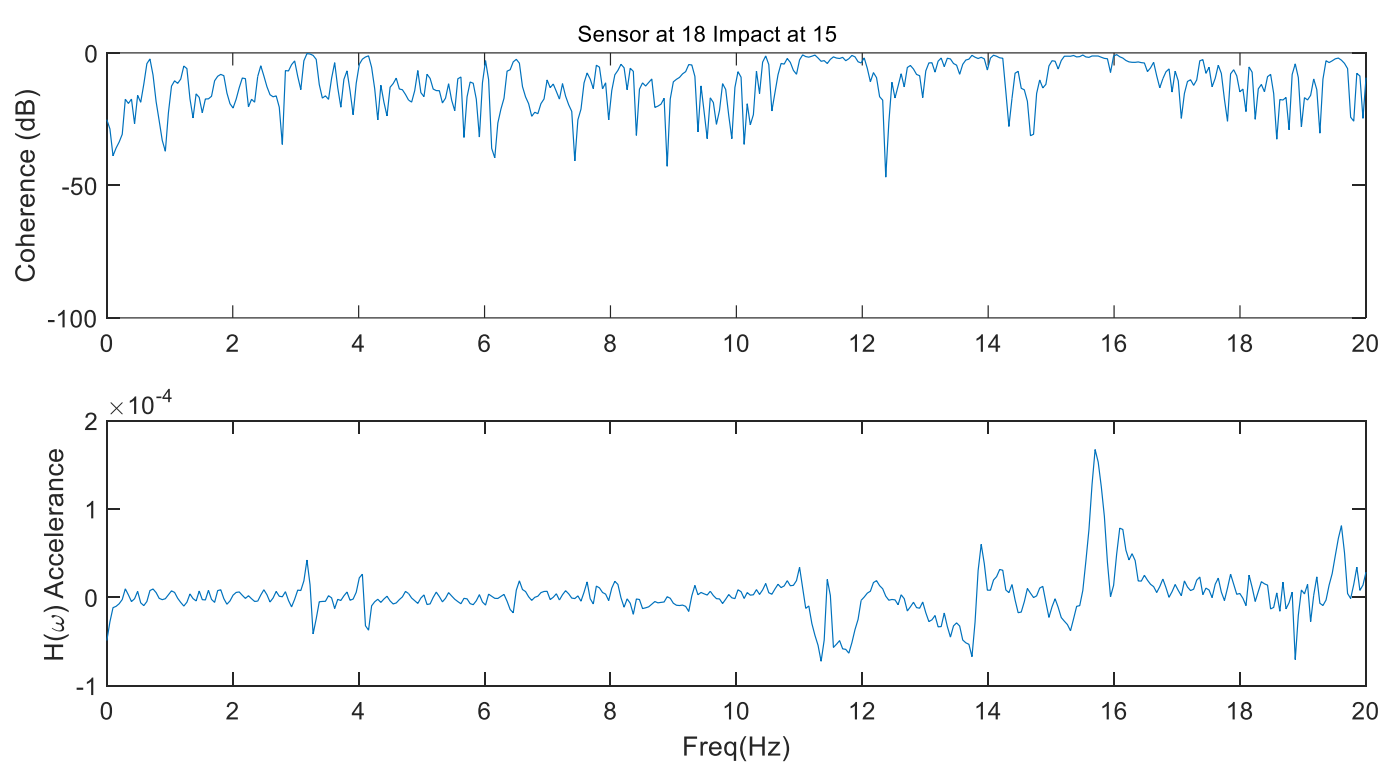

Figure 63. FRF from impact at location 15 and measurement at location 18 